\title{
A DESIGN METHODOLOGY FOR ROTORS OF SMALL MULTIROTOR VEHICLES
}

\author{
by \\ Timothy B. Carroll \\ B.Eng, Ryerson University, 2014 \\ A thesis \\ presented to Ryerson University \\ in partial fulfillment of the \\ requirements for the degree of \\ Master of Applied Science \\ in the Program of \\ Aerospace Engineering \\ Toronto, Ontario, Canada, 2017 \\ CTimothy B. Carroll 2017
}




\title{
A DESIGN METHODOLOGY FOR ROTORS OF SMALL MULTIROTOR VEHICLES
}

Timothy B. Carroll

Master of Applied Science, Aerospace Engineering, Ryerson University (2017)

\begin{abstract}
A model is presented for the aerodynamic performance prediction of fixed-pitch rotors for small unmanned aerial vehicles. The method uses a blade element momentum theory based approach that is formulated specifically for small rotors operating in hover and edgewise flight. In order to validate the model, a rotor test stand is used to measure the performance of a commercially available rotor for several inflow angles and advance ratios. The predictions agree with measurements for operating conditions excluding conditions with suspected vortex ring state. The model is incorporated into a numerical optimization scheme to demonstrate its potential as a design tool. Designs are presented that minimize the power loading for single- and multi-point operating conditions. The optimized designs have hyperbolic twist distributions, higher solidities, and operate at lower tip-speeds than existing designs. A potential flow based model is also presented to predict the wake interactions between multiple rotors in configuration.
\end{abstract}




\section{Acknowledgements}

Firstly, I would like to thank my supervisor, Götz Bramesfeld, for his suggestion to pursue a Master's degree and his guidance throughout the duration of this project. His unrelenting patience and effectiveness as an educator routinely helped me to transform many a fundamental concept from the abstract into practice. This also includes coordinating the industrial partnerships for which we could apply this research and for providing funding to help cover some of the educational expenses.

Much appreciation also goes to Mike Peasgood, Michael Tribou and Dmytro Silin at Aeryon Labs for helping to support this project and for sharing an abundance of industry feedback. I learned a great deal about the commercial approach to this type of project and am thankful for the opportunity to have worked with them during the summer of 2015. Thanks also to the

Ontario Centres of Excellence and the Natural Sciences and Engineering Research Council of Canada for also supporting this work.

Additional thanks goes to the aerospace department support staff for manufacturing and assembling the test stand portion of the experimental apparatus, and for helping with its installation inside the wind tunnel. Finally, sincere recognition is deserved by the members of the Ryerson Applied Aerodynamics Laboratory of Flight who contributed a lot of hard work to get the test stand up and running, and who helped with a large portion of the data collection for this thesis. 


\section{Dedication}

This work is dedicated to my Mother and Father, to whom much credit is deserved for their perpetual support, life-long encouragement and for teaching the importance of hard work. Credit is also due to my canine writing companion, Memphis, who supplied considerable moral support during the writing of this thesis by spending an equal amount of time beside my desk and patiently waiting for his walk. 


\section{Table of Contents}

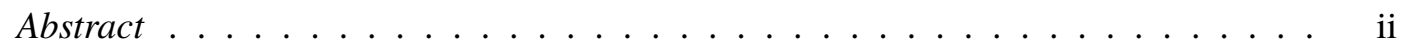

List of Tables . . . . . . . . . . . . . . . . . . . . viii

List of Figures . . . . . . . . . . . . . . . . . . . . . . ix

List of Appendices . . . . . . . . . . . . . . . . . . . . . xiii

List of Tables in the Appendices . . . . . . . . . . . . . . . . . xiv

List of Figures in the Appendices . . . . . . . . . . . . . . . . . . . . . xv

List of Symbols . . . . . . . . . . . . . . . . . . . . . . xvi

1 Introduction 1

1.1 Background . . . . . . . . . . . . . . . . . 1

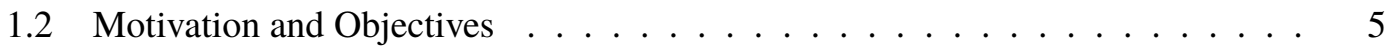

1.3 Organization of Thesis $\ldots \ldots \ldots \ldots \ldots$

2 Literature Review $\quad 7$

2.1 The Optimum Rotor . . . . . . . . . . . . . . . . . . . . . . . 7

2.2 Small Rotors and Propellers for Unmanned Aerial Vehicles . . . . . . . . . . 15

3 Methodology 21

3.1 Vehicle and Rotor Reference Frames . . . . . . . . . . . . . . . . . . . 22

3.2 Rotor Performance Coefficients . . . . . . . . . . . . . . . . . . . . 23

3.3 Blade Element Momentum Theory Based Approach . . . . . . . . . . . . . . 25

3.3.1 Blade Element Theory . . . . . . . . . . . . . . . . . 25

3.3.2 Momentum Theory . . . . . . . . . . . . . . . . . . 30

3.3.3 Approaches to Modelling the Rotor Inflow . . . . . . . . . . . . . . 31

3.3.4 Small Angles Approximations . . . . . . . . . . . . . . . . . . 31

3.3 .5 Large Angles . . . . . . . . . . . . . . . . . 36

3.3 .6 Forward Flight . . . . . . . . . . . . . . . . . . . . 39

3.3.7 Linear Inflow Models . . . . . . . . . . . . . . . . . . . . . . . 43 
3.3.8 Blade Section Aerodynamic Characteristics . . . . . . . . . . . . . . . 44

3.4 Vortex Wake Interaction Model . . . . . . . . . . . . . . . . . . . . . . 49

3.4 .1 Wake Induced Velocities . . . . . . . . . . . . . . . . . . 51

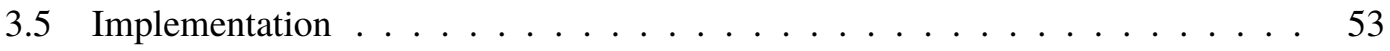

3.5.1 Performance Prediction Program . . . . . . . . . . . . . 53

3.5.2 Blade Geometry and Section Characteristics . . . . . . . . . . . . 57

4 Experimental Determination of Small Rotor Performance $\mathbf{5 8}$

4.1 Experimental Set-up . . . . . . . . . . . . . . . . 58

4.1 .1 Wind Tunnel Facility . . . . . . . . . . . . . . . . 61

4.1 .2 Testing Procedure . . . . . . . . . . . . . . . . . . 61

4.2 Test Stand Validation . . . . . . . . . . . . . . . . . . . . . . . . 62

4.3 Systematic Sources of Error . . . . . . . . . . . . . . 66

4.3.1 Signal Analysis and Vibrations . . . . . . . . . . . . . . . . . . 67

4.3 .2 Wind Tunnel Wall Interference . . . . . . . . . . . . . . . . 71

5 Validation of the Rotor Performance Prediction Methods $\quad 74$

5.1 Rotor Performance Model Validation . . . . . . . . . . . . . . . . . . . . . 75

$5.1 .1 \quad$ Hover . . . . . . . . . . . . . . . . . . . 75

5.1 .2 Edgewise Forward Flight . . . . . . . . . . . . . . . 76

5.1 .3 Azimuth Sensitivity _. . . . . . . . . . . . . 88

5.2 Rotor Performance Model Verification . . . . . . . . . . . . . . . . . . . . . 89

5.2.1 The Rotor Inflow Variation Due to Retreating/Advancing Blade Effects 89

5.2.2 Sectional Lift and Drag Coefficients . . . . . . . . . . . . . . . 91

5.2.3 Drag Divergence Mach Number and Compressibility . . . . . . . . . 93

5.2.4 Thrust, Power and Induced Drag Distributions . . . . . . . . . . . . 93

5.3 Vortex Wake Model Verification . . . . . . . . . . . . . . . . . 95

5.3.1 Wake Length and Ring Segmentation Effects . . . . . . . . . . . . 96

5.3 .2 Induced Velocities in Forward Flight . . . . . . . . . . . . . 97

5.3.3 Representation of the Single Rotor Flow Field . . . . . . . . . . . . 100

6 Sample Applications $\quad \mathbf{1 0 4}$

6.1 Rotor Design Using an Optimization Approach . . . . . . . . . . . . . . . 105

6.1 .1 Problem Formulation . . . . . . . . . . . . . . . . . . 105

6.1 .2 Optimization Algorithm _. . . . . . . . . . . . . . . 106

6.1.3 Implementation of the Optimization Approach . . . . . . . . . . 107

6.1 .4 Blade Parametrization . . . . . . . . . . . . . . . . . . 108 
6.2 Validation of the Approach . . . . . . . . . . . . . . . . 110

6.2.1 The Ideally Twisted Blade for Hover . . . . . . . . . . . . . . 110

6.3 Example Designs . . . . . . . . . . . . . . . . . . . . . . . . . . . . . 114

$6.3 .1 \quad$ Hover . . . . . . . . . . . . . . . . . . . . . . . 114

6.3 .2 Edgewise Flight . . . . . . . . . . . . . . . . . 117

6.3.3 Multi-objective Hover and Edgewise Flight . . . . . . . . . . . . 121

6.4 Modelling the Wake of a Quad-rotor . . . . . . . . . . . . . . 126

7 Conclusions 137

7.1 Conclusions . . . . . . . . . . . . . . . . . . . . . . . . 137

7.2 Future Work . . . . . . . . . . . . . . . . . . . . . 140

$\begin{array}{ll}\text { References } & 167\end{array}$ 


\section{List of Tables}

3.1 Rotor and propeller force and moment coefficients. . . . . . . . . . . . 23

3.2 Weighing factors for the first harmonic linear inflow distribution models. . . . . 44

6.1 Design variables and parameters for the rotor design problem. . . . . . . . 106

6.2 Design variables for the validation of the optimization approach. . . . . . . . . 110

6.3 Comparison of results obtained using several optimization approaches. . . . . . 113

6.4 Bound constraints on the design variables. . . . . . . . . . . . . . . . 115

6.5 Characteristics of two- and three-bladed rotor designs in hover. . . . . . . . . 116

6.6 Additional vehicle forces in edgewise cruising flight. . . . . . . . . . . 118

6.7 Characteristics of a three-bladed design for edgewise flight. . . . . . . . . . . 119

6.8 Characteristics of two- and three-bladed designs for hover and edgewise flight. . 122

6.9 Single point performance for the multi-point designs. . . . . . . . . . . . 125

6.10 Comparison between the multi- and single-point designs. . . . . . . . . . . 126 


\section{List of Figures}

1.1 The Aeryon SkyRanger sUAV platform . . . . . . . . . . . . . . 2

1.2 First person view $(\mathrm{FPV})$ racing drone $\ldots \ldots \ldots \ldots$

2.1 The Betz-Prandtl and Goldstein circulation distributions f . . . . . . . . . 8

2.2 Representation of the rotor wake structure . . . . . . . . . . . . . . . 9

2.3 Radial inflow distributions for several blade twist distributions . . . . . . . . . 11

2.4 Measurements of MAV rotor hover performance . . . . . . . . . . . . . . 18

3.1 Rotor and vehicle coordinate systems . . . . . . . . . . . . . . . . 22

3.2 Flow environment on the rotor blade and blade element . . . . . . . . . . . 26

3.3 Lift coefficient for the MA409 . . . . . . . . . . . . . . . . . . . . 45

3.4 Drag coefficient for the MA409 . . . . . . . . . . . . . . 46

3.5 Moment coefficient for the MA409 . . . . . . . . . . . . . . . . . . 46

3.6 Lift, drag and moment coefficients for a $\pm 180^{\circ}$ angle of attack . . . . . . . . 49

3.7 Vortex segment and wake geometry . . . . . . . . . . . . 52

3.8 Inflow routine block diagram. . . . . . . . . . . . . . . . . . . 54

3.9 Performance program routine 1: Input shaft speed. . . . . . . . . . 55

3.10 Performance program routine 2: Required thrust. . . . . . . . . . . . 56

3.11 Three dimensional computer model of a rotor blade . . . . . . . . . . . . . 57

4.1 Rotor test stand installed in wind tunnel test section. . . . . . . . . . . . . . . . 59

4.2 Test stand apparatus with components labelled . . . . . . . . . . . . . 60

4.3 Comparison of measured static thrusts . . . . . . . . . . . . . . 63

4.4 Comparison of measured static thrust coefficients . . . . . . . . . . . . 63

4.5 Comparison of measured static power . . . . . . . . . . . . . 64

4.6 Comparison of measured static power coefficient . . . . . . . . . . . 64

4.7 Comparisons of measured thrust coefficient . . . . . . . . . . . . . 65

4.8 Comparisons of measured power coefficient $\ldots \ldots \ldots 6$ 
4.9 Representative raw signal for the thrust load cell. . . . . . . . . . . . . . . 68

4.10 Representative raw signal for the torque load cell. . . . . . . . . . . . . . . 69

4.11 Representative raw signal for the pitching moment load cell. . . . . . . . . . . 69

4.12 Harmonic vibration amplitudes observed by the thrust load cell. . . . . . . . 70

4.13 Harmonic vibration amplitudes observed by the torque load cell. . . . . . . . . 70

4.14 Harmonic vibration amplitudes observed by the pitching moment load cell. . . 71

4.15 Representation of the rotor test stand oriented for edgewise flight. . . . . . . . 72

4.16 Wind tunnel wall interference effects. . . . . . . . . . . . . . . 72

5.1 Hover performance predictions using several inflow models. . . . . . . . . 75

5.2 Thrust predictions for the T-motor $18 \times 6.1$ at several angles of attack, $R P M=3000.77$

5.3 Thrust predictions for the T-motor $18 \times 6.1$ at several angles of attack, $R P M=4000.78$

5.4 Thrust predictions for the T-motor $18 \times 6.1$ at several angles of attack, RPM $=5000.79$

5.5 Power predictions for the T-motor $18 \times 6.1$ at several angles of attack, RPM $=3000.81$

5.6 Power predictions for the T-motor $18 \times 6.1$ at several angles of attack, RPM $=4000.82$

5.7 Power predictions for the T-motor $18 \times 6.1$ at several angles of attack, $\mathrm{RPM}=5000.83$

5.8 Power predictions for the T-motor $18 \times 6.1$ for $\alpha=90^{\circ}$ and $\alpha=60^{\circ} \ldots \ldots$. . . 85

5.9 Power predictions for the T-motor $18 \times 6.1$ for $\alpha=30^{\circ}$ and $\alpha=15^{\circ} \ldots \ldots$. . . . 86

5.10 Power predictions for the T-motor $18 \times 6.1$ for $\alpha=5^{\circ}$ and $\alpha=0^{\circ}$. . . . . . . . 87

5.11 Induced power ratio versus advance ratio in forward flight. . . . . . . . . . . 88

5.12 Azimuth station convergence behaviour on the thrust coefficient. . . . . . . . . 89

5.13 Elemental flow environment in the reverse flow region. . . . . . . . . . . . 90

5.14 Section inflow angle distribution for $\mu=0.25$ and $\mu=0.5 \ldots \ldots$. . . . 90

5.15 Section effective angle of attack distribution for $\mu=0.25$ and $\mu=0.5$. . . . 91

5.16 Section lift coefficient distribution for $\mu=0.25$ and $\mu=0.5$. . . . . . . . 92

5.17 Section drag coefficient distribution for $\mu=0.25$ and $\mu=0.5$. . . . . . . . 92

5.18 Section Mach number distribution for $\mu=0.25$ and $\mu=0.5$. . . . . . . . . 93

5.19 Section thrust distribution for $\mu=0.25$ and $\mu=0.5 \ldots \ldots \ldots$. . . . . . . 94

5.20 Section power distribution for $\mu=0.25$ and $\mu=0.5 \ldots \ldots \ldots$

5.21 Section induced drag distribution for $\mu=0.25$ and $\mu=0.5 \ldots \ldots$. . . . . . 95

5.22 Convergence study for ring wake elements. . . . . . . . . . . . . . 96

5.23 Convergence study for ring segments. . . . . . . . . . . . . . . . . 97

5.24 Induced velocity distributions along the rotor plane for $\chi=0^{\circ} \ldots \ldots \ldots 8$

5.25 Induced velocity distributions along the rotor plane for $\chi=14.04^{\circ} \ldots \ldots$. . . 98

5.26 Induced velocity distributions along the rotor plane for $\chi=26.56^{\circ} \ldots$. . . . . . 99

5.27 Induced velocity distributions along the rotor plane for $\chi=45^{\circ} \ldots \ldots$. . . . . 99 
5.28 Lines of constant induced velocity for a single rotor flow field for $\chi=0^{\circ} \ldots \ldots$

5.29 Lines of constant induced velocity for a single rotor flow field for $\chi=14.04^{\circ}$. . 101

5.30 Lines of constant induced velocity for a single rotor flow field for $\chi=26.56^{\circ}$. $\quad 102$

5.31 Lines of constant induced velocity for a single rotor flow field for $\chi=45^{\circ}$. . . 102

5.32 Lines of constant induced velocity for a single rotor flow field for $\chi=63.43^{\circ}$. . 103

5.33 Lines of constant induced velocity for a single rotor flow field for $\chi=75.97^{\circ}$. 103

6.1 Optimization approach flow chart. . . . . . . . . . . . . . . . . 108

6.2 Blade chord and twist distributions. . . . . . . . . . . . . . . . . . . . 109

6.3 Optimization for twist distribution using the nested genetic algorithm . . . . . . 111

6.4 Optimization for twist distribution using the constrained genetic algorithm . . . 112

6.5 Optimized twist distributions generated by GA and PSO algorithms . . . . . 113

6.6 Optimizated rotor geometries for hover. . . . . . . . . . . . . . . . . 116

6.7 Rotor trim in forward flight. . . . . . . . . . . . . . . . . . 117

6.8 Optimized designs for minimum power in edgewise flight. . . . . . . . . . . 119

6.9 Section thrust for the optimized and baseline rotors. . . . . . . . . . . . . . 120

6.10 Section power for the optimized and baseline rotors. . . . . . . . . . . . 120

6.11 Section induced power for the optimized and baseline rotors. . . . . . . . . . 121

6.12 Optimized designs for minimum power in hover and edgewise flight. . . . . . . 122

6.13 Optimized inflow distributions for minimum power in hover and edgewise flight. 123

6.14 Section thrust for the multipoint design case . . . . . . . . . . . . . 124

6.15 Section power for the multipoint design case . . . . . . . . . . . . . . . . . 124

6.16 Section induced power for the multipoint design case . . . . . . . . . . 125

6.17 Induced velocity ratio, $\frac{v_{i}}{v}$, from adjacent rotors for $\chi=0^{\circ}$ (diamond). . . . . 128

6.18 Normalized induced velocity contours from adjacent rotors for $\chi=0^{\circ}$. . . . . 128

6.19 Induced velocity ratio, $\frac{v_{i}}{v}$, from adjacent rotors for $\chi=14.04^{\circ}$ (diamond). . . . 129

6.20 Normalized induced velocity contours from adjacent rotors for $\chi=14.04^{\circ}$. . . . 129

6.21 Induced velocity ratio, $\frac{v_{i}}{v}$, from adjacent rotors for $\chi=26.56^{\circ}$ (diamond). . . . 130

6.22 Normalized induced velocity contours from adjacent rotors for $\chi=26.56^{\circ} \ldots 130$

6.23 Induced velocity ratio, $\frac{v_{i}}{v}$, from adjacent rotors for $\chi=45^{\circ}$ (diamond). . . . . 131

6.24 Normalized induced velocity contours from adjacent rotors for $\chi=45^{\circ}$. . . . 131

6.25 Induced velocity ratio, $\frac{v_{i}}{v}$, from adjacent rotors for $\chi=63.43^{\circ}$ (diamond). . . . 132

6.26 Normalized induced velocity contours from adjacent rotors for $\chi=63.43^{\circ}$. . . 132

6.27 Induced velocity ratio, $\frac{v_{i}}{v}$, from adjacent rotors for $\chi=14.04^{\circ}$ (square). . . . . 133

6.28 Normalized induced velocity contours from adjacent rotors for $\chi=14.04^{\circ}$. . . 133

6.29 Induced velocity ratio, $\frac{v_{i}}{v}$, from adjacent rotors for $\chi=26.56^{\circ}$ (square). . . . . 134 
6.30 Normalized induced velocity contours from adjacent rotors for $\chi=26.56^{\circ}$. . 134

6.31 Induced velocity ratio, $\frac{v_{i}}{v}$, from adjacent rotors for $\chi=45^{\circ}$ (square). . . . . . 135

6.32 Normalized induced velocity contours from adjacent rotors for $\chi=45^{\circ}$. . . . 135

6.33 Induced velocity ratio, $\frac{v_{i}}{v}$, from adjacent rotors for $\chi=63.43^{\circ}$ (square). . . . 136

6.34 Normalized induced velocity contours from adjacent rotors for $\chi=63.43^{\circ} \ldots \ldots 136$ 


\section{List of Appendices}

$\begin{array}{ll}\text { Appendix A } & \mathbf{1 4 3}\end{array}$

A.1 Force and Moment Coefficients of the Rotor and Propeller Conventions . . . . 143

A.2 Rotor and Propeller Geometries . . . . . . . . . . . . . . . . 145

A.3 Rotor Test Data . . . . . . . . . . . . . . . . . . . . . . . . 146

Appendix B $\quad \mathbf{1 5 5}$

B.1 Test Stand Components . . . . . . . . . . . . . . . . . . 155

B.1.1 Test Stand Uncertainty Analysis . . . . . . . . . . . . . . . 156

B.2 Ryerson University's Low Speed Wind Tunnel . . . . . . . . . . . . . . 157

$\begin{array}{ll}\text { Appendix C } & 158\end{array}$

C.1 Analytical Solution to the Single Rotor Flow Field . . . . . . . . . . . . . . 158 


\section{List of Tables in the Appendices}

B.1.1 Components used for the rotor test stand . . . . . . . . . . . . . . . . 155

B.1.2 Components used for the rotor test stand data acquisition system. . . . . . . 156

B.1.3 Uncertainty analysis summary for the test stand sensors. . . . . . . . . . . 156

B.1.4 Uncertainty analysis summary for the measured quantities. . . . . . . . . 156 


\section{List of Figures in the Appendices}

A.2.1 Sectional chord and pitch distributions for the MA11x7E . . . . . . . 145

A.2.2 Sectional chord and pitch distributions for the T-motor $18 \times 6.1 \ldots \ldots$

A.3.1 $C_{T}$ versus advance ratio for the T-motor $18 \times 6.1, \mathrm{RPM}=3000 \ldots \ldots$

A.3.2 $C_{T}$ versus advance ratio for the T-motor $18 \times 6.1, \mathrm{RPM}=4000 \ldots \ldots \ldots$

A.3.3 $C_{T}$ versus advance ratio for the T-motor $18 \times 6.1, \mathrm{RPM}=5000 \ldots \ldots \ldots$

A.3.4 $C_{P}$ versus advance ratio for the T-motor 18x6.1, RPM $=3000 \ldots \ldots \ldots$

A.3.5 $C_{P}$ versus advance ratio for the T-motor $18 \times 6.1, \mathrm{RPM}=4000 \ldots \ldots \ldots$

A.3.6 $C_{P}$ versus advance ratio for the T-motor $18 \times 6.1, \mathrm{RPM}=5000 \ldots \ldots \ldots 151$

A.3.7 $C_{M_{x}}$ versus advance ratio for the T-motor $18 \times 6.1, \mathrm{RPM}=3000 \ldots \ldots \ldots$

A.3.8 $C_{M_{x}}$ versus advance ratio for the T-motor $18 x 6.1, \mathrm{RPM}=4000 \ldots \ldots$

A.3.9 $C_{M_{x}}$ versus advance ratio for the T-motor $18 \times 6.1, \mathrm{RPM}=5000 \ldots \ldots \ldots$

B.2.1 Schematic of the RU closed circuit wind tunnel . . . . . . . . . . . . 157

C.1.1 Analytical resut for the induced velocities of single rotor flow field for $\chi=0^{\circ} \quad 158$

C.1.2 Analytical resut for the induced velocities of single rotor flow field for $\chi=14.04^{\circ} 159$

C.1.3 Analytical resut for the induced velocities of single rotor flow field for $\chi=26.56^{\circ} 159$

C.1.4 Analytical resut for the induced velocities of single rotor flow field for $\chi=45^{\circ} 160$

C.1.5 Analytical resut for the induced velocities of single rotor flow field for $\chi=63.43^{\circ} 160$

C.1.6 Analytical resut for the induced velocities of single rotor flow field for $\chi=75.97^{\circ} 161$ 


\section{List of Symbols}

A Area of the rotor disk

$C_{F_{x}} \quad$ Longitudinal force coefficient, $\frac{F_{x}}{\rho A(\Omega R)^{2}}$

$C_{F_{y}} \quad$ Lateral side force coefficient, $\frac{F_{y}}{\rho A(\Omega R)^{2}}$

$C_{M_{x}} \quad$ Rolling moment coefficient, $\frac{M_{x}}{\rho A \Omega^{2} R^{3}}$

$C_{M_{y}} \quad$ Pitching moment coefficient, $\frac{M_{y}}{\rho A \Omega^{2} R^{3}}$

$C_{P} \quad$ Power coefficient, $\frac{P}{\rho A(\Omega R)^{3}}$

$C_{Q} \quad$ Torque coefficient, $\frac{Q}{\rho A \Omega^{2} R^{3}}$

$C_{T} \quad$ Thrust coefficient, $\frac{T}{\rho A(\Omega R)^{2}}$

$\frac{C_{T}}{\sigma} \quad$ Blade loading coefficient

c Chord length

$c_{d} \quad$ Sectional drag coefficient, $\frac{d D}{q_{\infty} c}$

$c_{l} \quad$ Sectional lift coefficient, $\frac{d L}{q_{\infty} c}$

$c_{l, \alpha} \quad$ Lift curve slope

$c_{m} \quad$ Sectional moment coefficient about the airfoil quarter-chord location, $\frac{d M}{q_{\infty} c^{2}}$

$c_{n} \quad$ Normal sectional force coefficient

$c_{t} \quad$ Tangential sectional force coefficient

D Drag force

F Prandtl's tip-loss factor 
FM Figure of merit, $\frac{C_{T}^{3 / 2}}{\sqrt{2} C_{P}}$

$F_{\mathrm{X}} \quad$ Rotor force along $\mathrm{x}_{\mathrm{TPP}}$-axis

$F_{\mathrm{y}} \quad$ Rotor force along $\mathrm{y}_{\mathrm{TPP}}$-axis

$J \quad$ Propeller advance ratio

$L \quad$ Lift force

$\dot{m} \quad$ Mass flow rate

M Mach number

$M_{\mathrm{x}} \quad$ Rotor moment about $\mathrm{x}_{\mathrm{TPP}}$-axis

$M_{\mathrm{y}} \quad$ Rotor moment about $\mathrm{y}_{\mathrm{TPP}}$-axis

$N_{b} \quad$ Number of blades

$P \quad$ Power

$Q \quad$ Torque

$q_{\infty} \quad$ Freestream dynamic pressure, $\frac{1}{2} \rho V_{\infty}^{2}$

$R \quad$ Rotor radius

$r \quad$ Nondimensional radial position along the blade

$T \quad$ Thrust

$\frac{T}{P} \quad$ Power loading

$u_{i} \quad$ Swirl velocity, in-plane toward the rotor blade

$V_{A} \quad$ Axial velocity component towards the rotor plane, $V_{\infty} \sin \alpha+v_{i}$

$V_{\infty} \quad$ Freestream velocity

$v_{i} \quad$ Induced velocity, normal to the rotor disk

$V_{R} \quad$ Resultant velocity at the rotor plane, $\sqrt{V_{T}^{2}+V_{A}^{2}}$

$V_{\text {radial }}$ Radial component of the freestream velocity 
$V_{T} \quad$ Tangential velocity component along the rotor plane, $V_{\infty} \cos \alpha \sin \psi+\Omega y-u_{i}$

w Slipstream velocity in the far wake

$y \quad$ Radial distance along the blade

$\Gamma \quad$ Circulation

$\Lambda \quad$ Sweep angle

$\Omega \quad$ Angular velocity

$\Phi \quad$ Vehicle's roll angle

$\alpha_{0} \quad$ Zero-lift angle of attack

$\alpha_{\text {eff }} \quad$ Effective angle of attack

$\beta \quad$ Sectional angle of attack

$\chi \quad$ Wake skew angle, $\tan \chi=\left(\frac{\mu_{x}}{\mu_{z}+\lambda_{i}}\right)$

$\gamma \quad$ Cant angle of the vehicle's rotor support arm

$\lambda \quad$ Inflow ratio, $\frac{V_{A}}{\Omega R}$

$\lambda_{\infty} \quad$ Freestream inflow ratio, $\frac{V_{\infty} \sin \alpha}{\Omega R}$

$\lambda_{i} \quad$ Induced inflow ratio, $\frac{v_{i}}{\Omega R}$

$\mu_{\infty} \quad$ Freestream advance ratio, $\frac{V_{\infty}}{\Omega R}$

$\mu_{x} \quad$ Advance ratio parallel to the rotor, $\frac{V_{\infty} \cos \alpha}{\Omega R}$

$\mu_{z} \quad$ Advance ratio perpendicular to the rotor, $\frac{V_{\infty} \sin \alpha}{\Omega R}$

$\phi \quad$ Inflow angle, $\tan \phi=\frac{V_{T}}{V_{A}}$

$\psi \quad$ Blade's azimuth angle

$\rho \quad$ Air density

$\sigma \quad$ Rotor solidity (global), $\frac{N_{b} c}{\pi R}$

$\sigma_{l} \quad$ Local chord solidity, $\frac{N_{b} c(r)}{2 \pi y}$ 
$\varepsilon \quad$ Twist angle of the vehicle's rotor support arm

$\xi \quad$ Swirl ratio, $\frac{\Omega y-u_{i}}{\Omega R}$

$\xi_{i} \quad$ Induced swirl ratio, $\frac{u_{i}}{\Omega R}$ 


\section{Chapter 1}

\section{Introduction}

\subsection{Background}

The increase in popularity of small unmanned aerial vehicles (sUAVs) has led to the need for more efficient propulsion and lift systems. A popular sub-class among sUAVs are multirotors, which use multiple fixed-pitch rotors in various configurations to generate the propulsive forces required for controlled flight. An example of such a system is the Aeryon Labs SkyRanger, shown in Fig.1.1, which is a sUAV platform consisting of four rotors in quad-configuration and having a weight of 2.4 kilograms without payload. Vehicles of this nature typically have an operational endurance of up to 50 minutes and routinely perform remote sensing missions in support of search and rescue, disaster surveying, traffic collision reconstruction, and wildlife surveillance.

Advancements in the way of high energy-capacity-to-weight batteries, brushless electric motors, telecommunication systems, flight controllers, and avionics miniaturization have led to the popularization of multirotor sUAVs for commercial, civilian and recreational applications [1]. The primary advantage of using rotorcraft, as opposed to fixed wing UAVs, is based on their operational versatility as these systems can transition quickly between edgewise and hovering flight, can deploy and land without a runway, and can maneuver within confined areas. For these reasons, they are also used frequently in place of piloted vehicles that are incapable of executing dangerous missions cost effectively and safely [2]. 
It is arguable, as of late, that some of the aforementioned technological advancements have diminished the role that aerodynamic considerations play in the design of many commercial sUAV platforms. Several of the historically fundamental problems associated with conventional rotorcraft designs are mitigated using alternative means. For example, many aerodynamically unstable configurations can be piloted using intelligent flight control systems that utilize autonomous control and stability augmentation, and counteracting the rotor-torque reaction can be accomplished by having multiple rotors in configuration with little added weight penalty. Despite this, however, many of the performance limiting issues encountered with multirotor systems, such as the design of more efficient rotors, retreating blade effects in edgewise flight, the vortex ring state during slow vertical descents, and high altitude flight, remain problems which must be addressed using an aerodynamics based approach. Furthermore, the propulsion and lift system of a multirotor sUAV is often the largest power draw, in addition to being the main source of vibrations and acoustic noise, and thus, the aerodynamic design choices play a considerable role in the collective multidisciplinary performance of such vehicles.

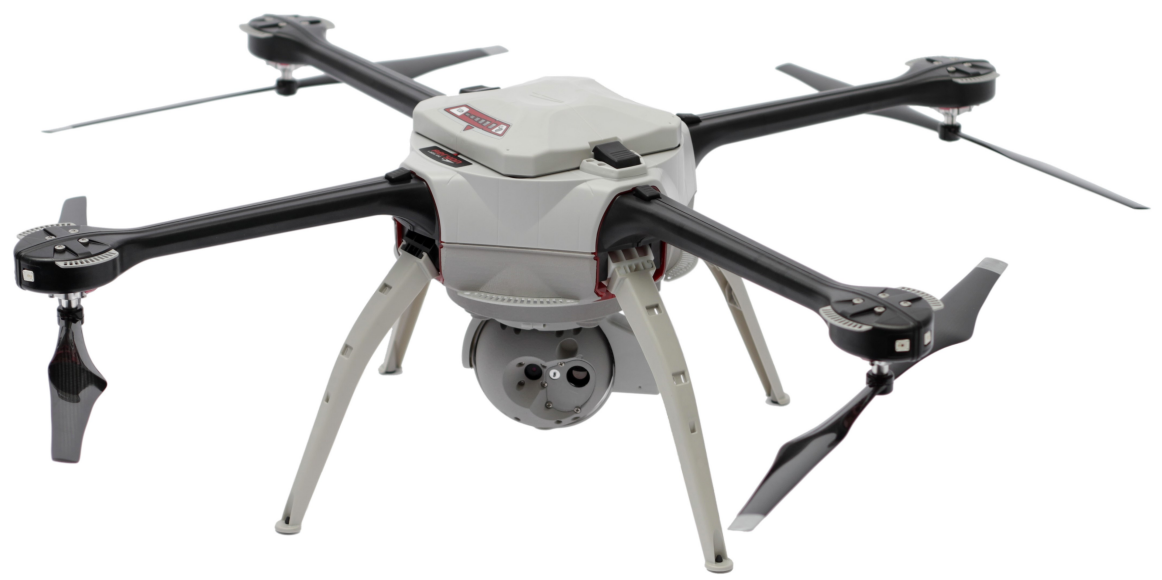

Figure 1.1: Example of a commercial sUAV platform: The Aeryon Labs SkyRanger [3].

For all classes of rotorcraft, designing the main rotor systems presents a unique challenge due to the competing design requirements for several operational modes and vehicle performance objectives. Such is the case when comparing the rotors used on the vehicle shown in Fig.1.1, that 
are designed to maximize hover endurance, versus the ones used on a first-person view racing drone, that are designed to maximize the propulsive forces in edgewise flight. First-person view (FPV) racing drones, such as the one shown in Fig.1.2, competitively race around tracks at relatively high speeds while being controlled remotely with on-board cameras. One of the most fundamental competing rotor design requirements is the blade twist distribution that is required to maximize the rotor efficiency in the hover, axial, and edgewise flight conditions. Existing literature has shown that much less twist is desired in edgewise flight compared to in hover [4-8]. Some degree of compromise be accomplished by using variable pitch blades, such as the case with full-scale articulating rotors, that use collective pitch adjustments to transition between flight modes. Although adjusting the collective does not inherently resolve the entirety of the competing blade twist requirements, the rotor blade dynamics can be carefully tuned to allow for more efficient operation for several flight conditions. Unlike full-scale rotor systems, however, small multirotor vehicles typically have fixed blades and instead use rotational speed to trim. Consequentially, their design consists of finding blade designs which provide adequate performance at several flight states and rotational speeds, while maintaining a fixed pitch.

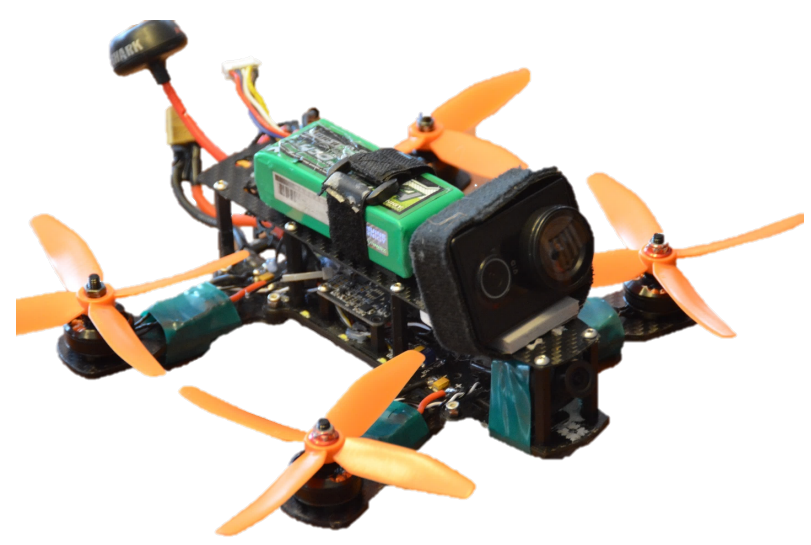

Figure 1.2: Example of a first person view (FPV) racing drone based on the Minion 220 frame by Rotorgeeks [9].

Another design consideration are the effects that the rotor configuration and disk sizing have on the performance of the collective rotor system. One example is that although having more rotors yields a more redundant system, maintaining an equivalent vehicle frame size requires 
that the diameter for each of the rotors is reduced. The interest therein lies in the aerodynamic effects associated with modifying the individual and cumulative disk loadings of the rotors. The rotor configuration also has ramifications on the wake induced interference effects caused by adjacent rotors, whereby a wake from one rotor modifies the inflow distribution of another. The associated performance effects with respect to selecting the rotor configuration, and whether or not the adjacent wakes induce significant interference velocities, has yet to be realized for sUAVs.

With respect to the future directions of sUAV research, Refs.10-12 summarize some of the challenges and planned milestones pertaining to rotorcraft development, and include ones for the small autonomous vehicle category. These consist of developing systems that operate more efficiently at low Reynolds numbers, improved multirotor vehicle range and endurance, and designing systems that can be manufactured at low-costs. A promising contemporary factor that may help to address some of these challenges is the application of computer aided design (CAD) and additive manufacturing techniques to small rotor design. Using such tools allows for designs to be built and tested within a very short time frame. Additive manufacturing is an attractive prototyping method for small rotors because of its higher cost efficiency compared with other methods like injection moulding or milling. Furthermore, some of the more recently available build materials, such as fibre-reinforced polymers and composites, have high elastic and shear moduli meaning that the ratio of the material strength to the blade's expected internal stresses is significantly higher compared to full-scale rotor blades. For this reason, more complicated chord solidities and twist distributions can be investigated. Moreover, the reduced time frame between the conceptualization, prototyping and experimental testing means that the new rotor designs, and the predictive limits of the simulation tools used to model them, can be more thoroughly investigated, especially with respect to designs for specific sUAV platforms and performance requirements. 


\subsection{Motivation and Objectives}

The purpose of this thesis is to introduce a design-build-test stream for small rotors that consists of a performance prediction model, design methodology, and an experimental testing apparatus. In doing so, further understanding of the aerodynamic behaviour of small fixed pitch rotors can be achieved, with particular focus on rotor designs that compromise between the requirements for hover and edgewise flight. To this end, an aerodynamic model is formulated in order to predict the loads and induced velocities on small rotors. The model captures some of the effects associated with low chord-Reynolds numbers and some of the effects associated with the retreating blade and highly twisted inboard blade sections. The model is validated using experimental results encompassing a wide range of operating conditions that encompass the performance limits representative of current multirotor sUAVs. Finally, the model is implemented within a numerical optimization framework which is used as a design tool.

Multiple sources have pointed out the continued need to study small scale rotors and the emerging market potential of remotely piloted or autonomous sUAVs [10-13]. Having a designbuild-test stream is useful for validating the methodologies that are used to model their performance, for comparing the performances between multiple hypothetical designs, for verifying the experimental performances of existing designs, and for creating new designs that have specific design requirements and constraints. Though there exist some similar design and testing frameworks, such as ones applied to micro-scale rotors [14,15], small UAV propellers [16-18] and small coaxial rotors in hover and vertical flight [19], these do not encompass the edgewise flight condition. Subsequently, there is also a lack of published experimental performance data for small rotors operating in edgewise flight. Therefore, an additional objective of this thesis is to present a compilation of wind tunnel performance data for a commercially available rotor for several angles of attack and advance ratios.

\subsection{Organization of Thesis}

This thesis has seven chapters. Chapter 2 consists of a formal literature review that summarizes the historical foundations of theories used to model rotor performance. This also includes 
a survey of some of the existing design methodologies small- and micro-scale propellers and rotors. In Chapter 3, a methodology is presented that is used to model the performance of a single rotor and the wake geometries of multiple rotors in configuration. This includes a description of how these models are implemented into a computer prediction program. In Chapter 4, a rotor test stand, data acquisition set-up and experimental testing methodology is presented. The measurements that are obtained using the apparatus are compared with measurements from another facility. The results generated using the rotor performance model are then compared with experimental measurements for the purpose of validation in Chapter 5. The model is implemented within an optimization framework so that it may be used as a rotor design tool. Chapter 6 discusses the formulation of the optimization problem in addition to the numerical methods used. Finally, the results from this thesis are concluded in Chapter 7, and include several recommendations for future research. 


\section{Chapter 2}

\section{Literature Review}

\subsection{The Optimum Rotor}

Designing an optimum rotor, one which operates at maximum efficiency, requires finding a radial blade loading that has minimum induced drag. A formalized attempt for determining this loading for aviation propellers was first developed by Betz, who modelled the propeller wake with a series of uniformly convecting rigid helical vortex sheets [20]. Much like Munk's elliptical loading for planar fixed wings [21], Betz hypothesized that a circulation distribution that produces a uniform downwash also has minimum induced losses. Although his approach was not an explicit optimization, Betz applied the principles of calculus of variations in order to determine the radial circulation distributions for propellers having an infinite number of blades. Prandtl extended Betz's result to propellers with a finite number of blades by deriving a tip-loss correction factor. Prandtl determined this correction factor by modelling the wake using two dimensional, axisymmetric slices in which the vortex sheets are represented using semi-infinite lines of vorticity [20]. Together the combination of these results is known as the Betz-Prandtl circulation distribution, and is represented by the dashed lines in Fig.2.1 which shows an ideally loaded, two-bladed propeller operating at several forward advance ratios. The vertical and horizontal axes, representing the circulation and radial span, respectively, are functions of the propeller advance velocity, $v$, its rotational speed, $\Omega$, and the wake's slipstream velocity, $w$.

One of the drawbacks associated with the Betz-Prandtl radial circulation distribution is 
that the accuracy of the tip-loss correction factor is significantly diminished for propellers with fewer number of blades. Therefore, using the Betz-Prandtl circulation distribution as a presupposition, Goldstein derived an analytical solution for the optimum circulation distribution for lightly loaded propellers with a finite number of blades. This was done by representing the flow field with a bound vortex system that consists of curved vortex sheets [22]. Goldstein's solution is also shown in Fig.2.1 by the solid lines. As the advance ratio increases, the BetzPrandtl circulation distribution approaches Goldstein's result and both solutions simultaneously become more representative of the physical propeller wake. This is due to the propeller's selfinduced velocity becoming a smaller fraction of the wake's resultant axial velocity and, thus, the emanating vortex sheets remain as irrotational screw surfaces in both the near and far-fields. In essence, these wake models neglect any wake contraction and are valid for lightly loaded propellers.

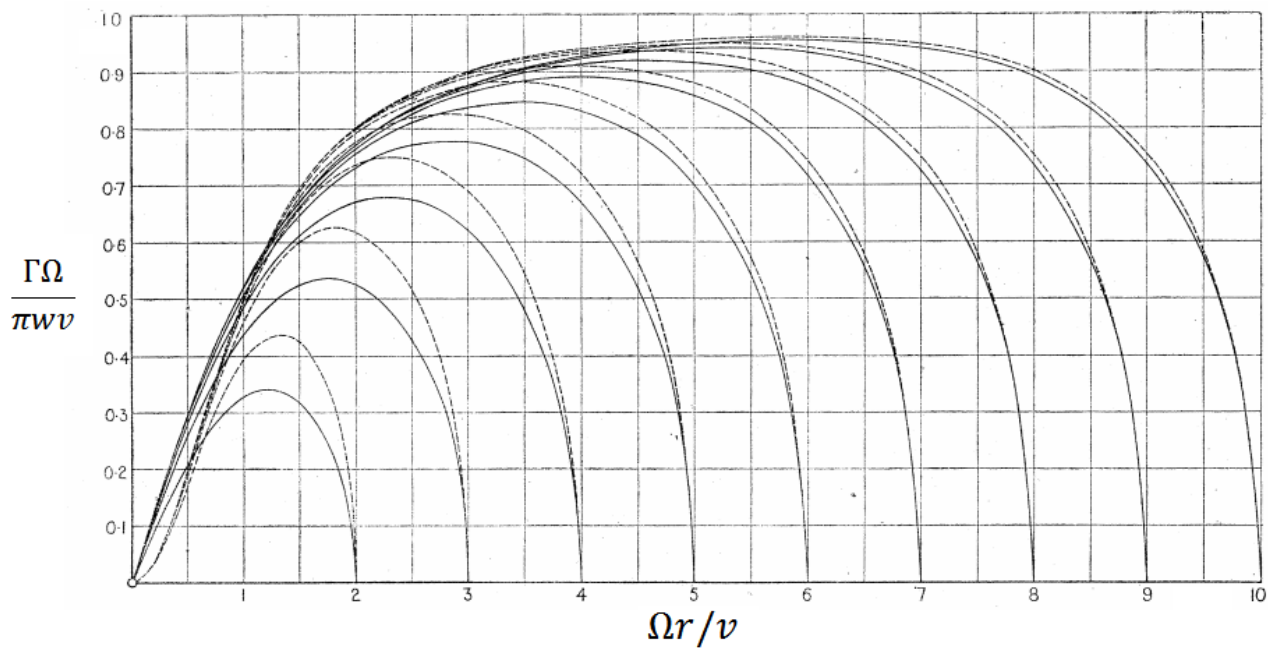

Figure 2.1: The Betz-Prandtl (dashed lines) and Goldstein (solid lines) radial circulation distributions for a two-bladed propeller operating at the minimum induced loss condition for several advance ratios.

Theodorsen addressed some of the lighly loaded assumptions of Goldstein's solution in order to model the wake contraction of heavily loaded propellers. He did this by introducing a mass coefficient and axial-loss factorin order to model the rigid wake in the far-field [23]. This forumlation also gives the solution for a static propeller, for which Theodorsen suggests 
there also exists a uniform induced velocity distribution over the propeller disk as well as throughout horizontal planes in the wake. However, recent results have shown his assumptions to be unrepresentative of a helicopter rotor wake in hover [24], primarily due to the fact that the blade's shed tip vortices contract and descend at a much slower rate than the blade's downwash. This is shown in Fig.2.2 which also depicts the vortex sheets contracting in the far wake. An additional point made by Theodorsen is that a blade's profile drag and induced loss are considered independently, so that in a design sense, the sectional characteristics can be selected to minimize profile losses once an idealized circulation distribution is determined.

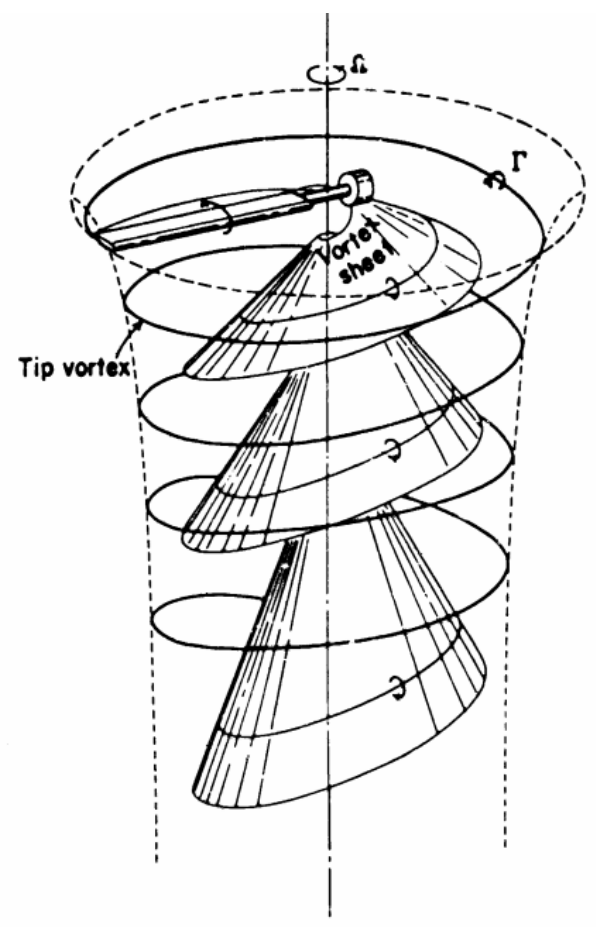

Figure 2.2: Representation of shed tip vortices and curved vortex sheets which constitute the rotor wake [25].

Concurrent to the development of the aforementioned propeller vortex wake models, blade element momentum theory was also being used to model airplane propeller performance ${ }^{1}$. Blade

\footnotetext{
${ }^{1}$ The foundations of propeller theory were laid in the nineteenth century for naval propellers with Froude's blade element theory [26] and Rankine's momentum theory [27].
} 
element momentum theory is a combination of the momentum and blade element theories. In momentum theory, the propeller is modelled as an actuator disk with uniform inflow [28]. Although giving an estimate for the propeller's self-induced velocity, the shape of the propeller blade is ignored. Thus, using uniform momentum theory on its own is only representative of propellers that produce uniform axial displacement velocities and that have no tip-losses. In blade element theory, the propeller blade is discretized into radially distributed two-dimensional sections. Though the forces along the blade can be easily found by summing the performance of the blade sections, this method gives no approximation of the blade's self-induced velocities. By combining momentum and blade element theories, however, both the induced velocities and forces acting on each blade element can be determined.

One of the first applications of blade element momentum theory to helicopter rotors was presented by Gustafson and Gessow [4]. The authors determined that a rotor blade with minimum induced losses in hover also has a uniform inflow across the blade, as shown in Fig.2.3. In order for this to occur, the blade must be twisted inversely with respect to its distance from the axis of rotation. Using the idealized twist distribution and the assumption of uniform inflow, they were able to study the conflicting tip speed requirements of a power limited rotor system in hover and forward flight. They found that for a given thrust, hover performance increases by lowering tip speed, provided that each blade section is operating at its best lift-to-drag. Determining an appropriate tip speed for single geared helicopters consists of a trade-off between the high tip speed requirements that are required to avoid tip stall at high forward-flight speeds and the low tip speeds that increase hovering efficiency. The authors suggest that there exists a compromise for a power limited system that maintains the maximum forward speed by means of using twisted blades. To demonstrate this, a rotor operating with a $12.4 \%$ reduction in tip speed was demonstrated to require $8.1 \%$ less power in hover and a $27 \%$ increase in rate of climb without limiting its initial design forward-flight speed. A similar trend was demonstrated for two rotor solidities. This is applicable to sUAV rotors since their brushless motors have both a single gear ratio and upper power limit, similar to the conditions described by Gustafson and Gessow. For single rotors, a consequence of the reduced tip speed is the rapid increase in the dissymmetry of lift due to tip stall in forward flight. Multirotor UAVs however, can to some degree maintain 
level flight after exceeding the tip stall limit due to the balancing effect from multiple rotors. Nonetheless, this remains a limiting operating condition due to the large power requirements associated with retreating blade effects.

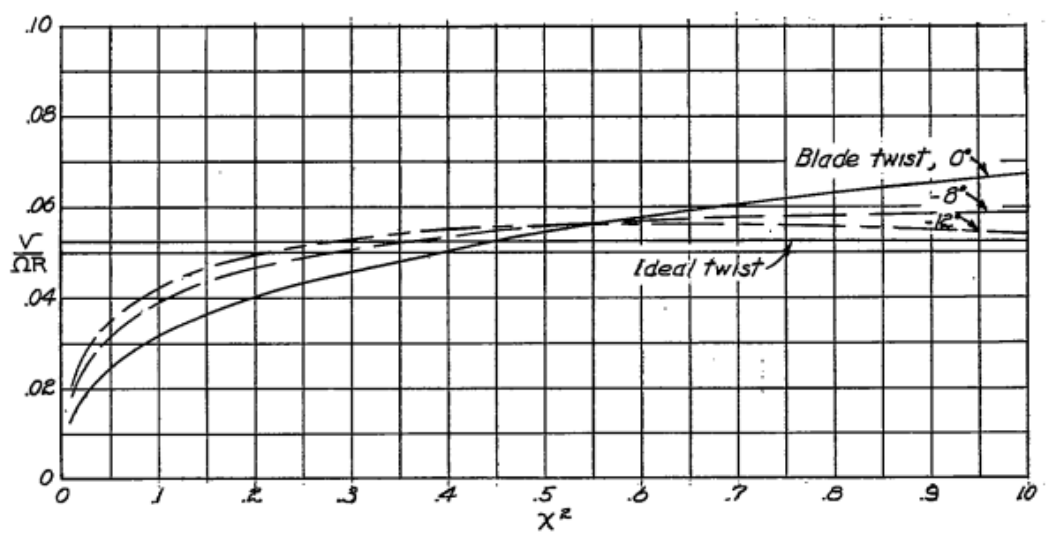

Figure 2.3: Radial inflow distributions corresponding to several blade twist distributions [29].

Gessow later used blade element momentum theory to explore the effects of blade twist and planform taper on a rotor's hover efficiency [29]. It was determined that for ideally twisted blades, the chord distribution that is required to minimize profile drag also varies hyperbolically. Thus, the classic optimum twist and chord distributions to minimize induced and viscous losses in hover are:

$$
\begin{gathered}
\beta(y)=\alpha_{\mathrm{eff}}+\frac{v_{i}}{\Omega y} \\
c(y)=\frac{c_{\mathrm{tip}}}{r}
\end{gathered}
$$

where the effective angle of attack, $\alpha_{\text {eff }}$, should coincide with the section's best lift-to-drag ratio. A simpler compromise to the hyperbolic distributions is found using linear twist and chord distributions, for which Gessow found that a 5\% increase in thrust is obtained using between a $-8^{\circ}$ and $-12^{\circ}$ linear twist rate distribution in combination with a 3:1 root-to-tip taper ratio. Furthermore, he found that the induced losses account for $80 \%$ of the total power required in hover and are minimized, in addition to the profile losses, by redistributing the blade loading 
towards the inner portion of the blade. This is because the a section's power is a function of the cube of the section velocity, whereas the thrust increases with the square of the velocity and, thus, it is more beneficial to increase the loading on the lower velocity sections. Investigation using blades having different solidities resulted in similar net performance improvements for the same twist and taper ratios, and for partial tapers. Furthermore, incorporating negative radial twist distributions can also help in forward flight to delay retreating blade effects at high speeds, in part, due to unloading of the tips.

Thus far, two methodologies have been presented to model propellers and rotors: a liftingline based approach, where the blade is modelled by a bound vortex system and the wake is modelled using helical sheets of vorticity, and a blade element momentum theory approach, that uses strip theory to model the blade and uniform momentum theory to model the wake. Though these methods give the radial loadings that are required for minimum induced losses, they do not explicitly describe a design procedure that can be used to obtain the blade geometries that achieve the ideal loadings, notwithstanding the hyperbolic twist and chord distributions in hover. As a result, several design procedures that are based on the findings of the previously discussed methods, have been since developed and are summarized herein.

Larrabee developed a formalized design procedure, using the minimum induced loss circulation distribution, to determine the optimum propeller geometry based on a desired operating point, number of blades and a required disk loading [30]. Later, Adkins and Liebeck modified this procedure by eliminating some of the small angle and lightly loaded assumptions and include a method for analysing simple off-design point geometries [31]. Using their design approach, they show that, similar to Theodorsen's proposition, the induced losses and profile power can be considered independently, assuming that the wake has only small variations in radial velocity. Thus, the choice of thickness and airfoil distribution can be considered as a final step in the design process. Conceivably, both of the aforementioned design algorithms can be considered to be optimization approaches since they minimize the induced losses, given explicit design parameters. Although they can be applied to designing small rotors for vertical flight, these two methods only make allowances for designs operating near to the idealized loading for the axial flight condition. Furthermore, explicit design constraints on the blade geometry are not included 
in the actual procedures. For example, Larrabee's inclusion of a "hub-smoothing" modification, to reduce interference drag around the fuselage, is performed after the initial design has been done, which begs to question if there exists a more efficient propeller geometry that incorporates this constraint into the design.

A drawback to the aforementioned design approaches is that they are formulated for singular performance operating points and are intended for a single objective, minimum losses. Often a more comprehensive design is required to encompass several flight states or include specific geometric constraints. For example, one might want to optimize for a compromise between hover and forward flight, include constraints on multidisciplinary performance metrics, or in the case of multirotor vehicles, encompass the aerodynamic effects of adjacent rotors. Numerical optimization methods, which have considerably grown in capability due to computational improvements, have found popularity with rotor design problems. This is largely because of the many multidisciplinary design aspects encompassing the rotor and power systems of a helicopter. However, some of these efforts have been applied to finding the optimal blade loading in forward flight and have also been used to explore hybrid design cases.

The optimal blade lift distribution for a rotor during hover and forward flight was investigated by Moffitt and Bissell who devised an approach similar to Theordorsen's, but extended to include a induced torque differential term [24]. The method was formulated to give a noniterative solution for the circulation distributions required for minimum induced power in hover. The model uses lightly loaded assumptions, neglects unsteady aerodynamic effects and blade flapping, and is supplied with prescribed wake geometries generated using Sikorsky's Circulation Coupled Hover Analysis Program. By optimizing the twist distribution of a UH-60 Black Hawk rotor for hover, the idealized uniform inflow distribution was shown for a large portion of the inboard blade, and is similar to the result obtained using blade element momentum theory. The exception being for the a sharp increase in circulation on the outboard blade which is caused by the descending shed tip vortices that contract to approximately $78 \%$ of the blade radius. The circulation distributions on the optimized blade accounted for this by imposing a localized twist spike at the tip. The twist requirements in forward flight for an azimuthally varying twisted blade was also investigates using a rigid helical wake model. The optimized circulation distribution 
resulted in $10 \%$ less required power compared to the baseline, where a reduction in induced power accounted for $34.7 \%$ of those power savings.

Bennett applied various numerical optimization techniques to the design of multiple rotor components, including the blade twist in hover [32]. The ideal inverse twist distribution described by Gessow was found to require the least power. For the analysis a simple blade element momentum approach, based on the one outlined in Ref.33, was used in combination with a non-linear programming algorithm. The method consisted of finding the twist distribution at 10 radial stations for a blade with a known planform, airfoil distribution, radius and constant tip speed.

Walsh et al., presented a formal optimization procedure for a rotor blade that minimizes hover power, while assuring adequate forward flight performance [34]. The motivation for the work was to demonstrate the potential that using mathematical optimization techniques present for reducing the design time of a main rotor system when compared to more conventional "by hand" approaches. The authors suggest that this methodology can be applied to designing rotor blades made from composite materials, which allow for more complex twist and chord distributions, compared to the conventional extruded aluminium blades. The approach uses two methods: strip theory for hover and a Rotorcraft Flight Simulation program (C81) to simulate a pull-up manoeuvre in forward flight. These are combined within the constrained minimization optimization algorithm program CONMIN. The approach used three design requirements that consisted of requiring the rotor to operate below the allowable power threshold during hover, no blade section stall except in the reverse flow region, and having the capability to trim during a simulated pull-up manoeuvre. The performance of their designs are comparable to the conventional ones, but were obtained in times on an order of magnitude faster.

Kroninger developed an approach to minimize the total power consumption of a rigid, hinge-less rotor. His method uses a coupled free wake and lifting line methodology to find the optimal circulation distributions during hover and forward flight [35]. The circulation for the optimized designs were allowed to vary radially and azimuthally as though it were a morphing rotor. A four-bladed rectangular rotor with $-6^{\circ}$ linear twist and operating in hover at was used as a baseline geometry. Modest performance gains in required power were found for advance 
ratios up to 0.4 , whereas a $30 \%$ reduction was found during hover. A reduction in induced power at all flight conditions was the main source of power savings, which at times was at the expense of higher profile power. The lift distributions tended to be redistributed inboard on the optimized rotors. The non-smooth twist distributions for the optimized blades resulted from the optimization routine attempting to smooth the radial lift distributions in areas that had erratic features in the wake, such as trailing tip vortices. The resulting lift distribution therefore varies non-linearly in the radial and azimuthal frames. A gradient-based optimization routine was used and, therefore, further validation is needed to determine if the optimized circulation distributions are ideal in forward flight.

Much of the work on rotor optimization within the last decade has been devoted to the multidisciplinary analysis of the entire rotor system, especially with respect to aeroelastic, acoustic and vibratory performance. Advanced aerodynamic prediction methodologies, such as those using potential flow or even computational fluid dynamics, have become more feasible due to numerical decoupling techniques, such as multilevel objective decomposition and the computational parallelization frameworks presented in Refs.8,36 and 37. The culmination of these efforts have resulted in advanced rotor designs that reduce vibratory loads, noise footprints and use dynamic aeroelastic tailoring. Nevertheless, most of this work has been focused on full-scale, articulating rotor blades and less so on small UAV rotor blades. Therefore, the accompanying section focuses on some of the current and ongoing work related specifically to design methodologies for small and micro-scale rotors.

\subsection{Small Rotors and Propellers for Unmanned Aerial Vehicles}

Compared to sub-scale rotors and small propellers, there has been relatively little focus on the analysis and design of small multicopter rotors. This is evident from the small amount of published data on their performance outside of hover and axial flight. Therefore, the purpose of this section is to summarize the existing literature related to small rotor design and to identify where this thesis fits in the existing body of knowledge.

A survey of the experimental performance trends for small and micro-scale rotary vehicles 
was published in Ref.12 for a number of vehicle configurations and commercially available small rotor blades for scale-model helicopters. The results focused on characterizing the propulsive efficiency and hover performance of low Reynolds number rotors and determining their induced power factors. The rotors had blades with both tapered and rectangular planforms with tip Reynolds numbers less than 40,000. The peak figure of merit, which is the ratio between the ideal power and actual power during hover, that was obtained for both sets of rotor blades ranged from 0.34 to 0.53 and are considerably lower than modern full-scale helicopter rotors. The induced power factor, which is a coefficient representing a rotor's viscous losses, was between 1.1 and 1.4 .

A number of publications from the University of Maryland examine the performance of rotors for micro aerial vehicles (MAVs) and identify a number of aerodynamics challenges related to their development $[11,14,15,38,39]$. Though a majority of the systems studied are much smaller compared to the ones in this thesis, many of the performance trends are also applicable.

Ref.14 summarizes the experimental performance of several micro aerial vehicle rotor designs in hover. The experiments consist of a parametric studies to investigate isolated performance related to airfoil selection, and the combined performances of coupling airfoil selection with different blade planforms, tip shapes and linear twist rates. The blades are designed to be used on the University of Maryland's micro-scale coaxial rotorcraft (MICOR) that can accommodate rotor diameters up to $22 \mathrm{~cm}$. The best performing blades were found to have a combination of a 2:1 taper ratio on the outer portion of the blade, a gradual negative twist rate (with a 18 to 22 degree collective pitch setting), and used circular arc airfoil sections with sharp leading edges. A significant performance factor was in the selection of the appropriate airfoils for low section Reynolds numbers where thin cambered plates achieved much higher lift-to-drag ratios than more conventional airfoils. The highest figure of merit obtained from single rotor tests was 0.65 , which is $20.6 \%$ lower compared to those which can be achieved with modern full-scale helicopters. The under-performance is attributed to the viscous effects associated with low section Reynolds numbers and were found to account for more than the $40 \%$ of losses for the micro-scale rotors. As a result, the lower solidity blades performed poorly despite having a 
higher blade loading coefficient, $\frac{C_{T}}{\sigma}$, which for full-scale rotors, correlates with better hovering performance. This suggests that at least for the inboard portion of micro-scale blades, the profile drag penalty associated with having a larger sectional chord, is less than the viscous drag penalty incurred by these sections operating at low Reynolds number.

For small rotors using variable tip speeds, this has a number of implications with respect to designs that operate at the maximum power loading, $\frac{T}{P}$. Without drastically changing the radial twist and chord distributions, maximizing the power loading is achieved by reducing the rotational speed and increasing the rotor solidity, which takes advantage of the cubic and square relationship of the power and thrust, respectively, to a section's velocity. Increasing the solidity can be achieved by either increasing the number of blades or the sectional chord. Adding blades is beneficial for reducing the induced tip losses, minimizing profile losses on the inboard blade and has secondary effects such as reducing the acoustic footprint and vibratory loads. However, the sectional chord lengths when using more blades is smaller and so the performance benefit is therefore limited by sectional Reynolds numbers which do not incur significant viscous losses.

Another important result from Ref.14 is that the peak figures of merit, obtained over several tested rotational speeds, coincide approximately at the same collective pitch setting. Therefore, to obtain the highest power loading for a required thrust, it is advantageous to trim via the rotor's rotational speed while maintaining a constant blade pitch, rather than using collective. This way, the optimal power loading is maintained across multiple rotational speed. This is the antithesis to full-scale rotors where typically the rotational speed is held constant and collective pitch is used in order to trim for the required thrust. This is observed in Fig.2.4, which shows that power loading versus the disk loading for several rotational speeds. Trimming to a different disk loading, in the rotational speed sense, can be visualized as descending along the gradient of the coalescing lines of constant rotational speed. Under ideal conditions, this follows the power loading envelope, that is the power loading at the maximum figure of merit, which is plotted in red. By comparison, trimming with collective would trace along the lines of constant rotational speed and, therefore, the optimum power loading coincides with a single disk loading for a system having a single gear ratio. 


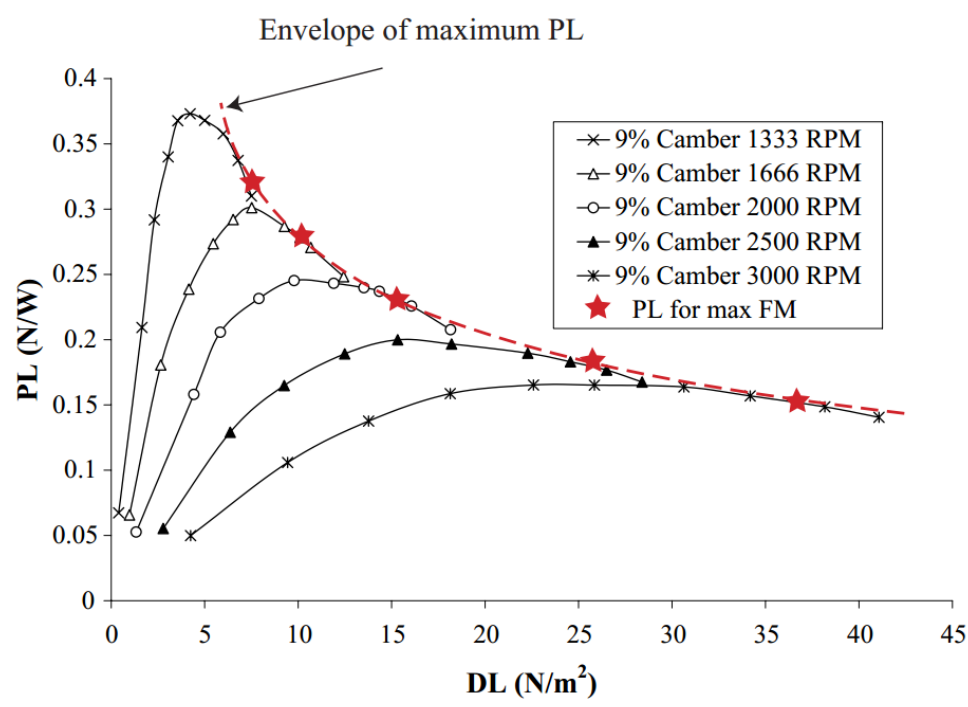

Figure 2.4: Power loading vs. disk loading at various rotational speeds for rectangular bladed rotor with $9 \%$ section chamber $(\sigma=$ $0.1279, R=11.2 \mathrm{~cm}, N_{b}=2$ ) [14].

Several literature sources investigate the performance of small scaled propellers using experimental testing $[17,40]$. For example, a compilation of commercially available hobby propeller performance data from tests at the University of Illinois at Urbana-Champaign's low speed wind tunnel is compiled in Ref.40. The database provides a good set of cases that are well suited for the validation of theoretical models for the propeller working state.

With respect to the design of small UAV propellers, Burger presents a methodology that considers their aerodynamic and aeroacousitc performance [41]. Using the framework of a genetic algorithm, several optimized propeller geometries were determined for three flight cases that include launch, fast axial flight, and a hybrid of the two. The aerodynamic prediction method used a vortex lattice method that modelled the propeller blade using vortex ring elements. In addition, the model included compressibility and viscous corrections. A class-shape transformation method was used to model the propeller geometry using Bernstein polynomial shape functions. Results showed differences in circulation distributions between the single and multipoint design cases, where the latter had a more smoothed spanwise distributions.

Gur and Rosen present a multidisciplinary propeller design approach for aerodynamic, struc- 
tural and acoustic performance of an ultra-light propeller [42] . A mixed optimization scheme that consisted of a genetic algorithm, enumerative scheme (simplex method), and gradient based method arranged in series, was used to determine the optimal geometry and operating conditions for six design cases. Empirical relations that characterized the engine efficiency and vehicle performance were implemented along with a number of structural, acoustic and aerodynamic constraints. A blade element momentum theory was used for the aerodynamics portion of the analysis method. The design variables consisted of the propeller's rotational speed, twist and chord distributions and the objective function was to minimize the engine fuel consumption. Optimal designs were compared to the radial twist distributions required to produce the idealized Betz-Prandlt loading. Amongst the majority of design cases, the propeller's twist distribution was primarily driven by aerodynamic considerations, whereas the thickness and chord distributions were modified based on accompanying structural and acoustic constraints. Two designs for minimum fuel flow and maximum flight speed resulted in different respective geometries that had much different performances. Compared to the low-speed design, the high-speed one used $24 \%$ more fuel in loiter, but its maximum airspeed increased by $29 \%$. The benefit of the design scheme was that it enabled a weighted combination of the two objectives for a single propeller design.

Sodja et al. present a study of the aeroelastics of flexible propellers that are optimized to prolong aerodynamic efficiency at higher advance ratios [16]. A structurally adaptive blade element momentum based model was used to capture the performance of the deflected blades. The objective function consisted of minimizing the curvature of the efficiency versus advance ratio relationship around a specified design point. An optimum deformed blade geometry was found through an iterative procedure, based on a response surface methodology. A design algorithm allowed for the determination of the corresponding undeformed blade geometry. The dynamics and the aeroelastic stability of the blades were not included in the procedure and the blade loading was treated as though it was comprised of static forces. Experimental testing was performed to validate the aerodynamic model and to predict the performance of the flexible blades. The tests confirmed that the efficiency envelope was extended at high advance ratios using aeroelastic tailoring. 
From this literature review, it is evident that in order to further understand the performance and design of small rotors, a number of challenges have to be addressed. First, a prediction method must be developed that accurately models the aerodynamic performance of smallscale rotors in edgewise flight. Second, more experimental test data is needed in order to demonstrate some of the performance limits of small rotors, especially over many tip speeds and angles of attack. This requires an experimental set-up that can also be used to validate the aforementioned performance prediction model. Finally, the wake interference effects resulting from multiple rotors in configuration must be investigated in order to determine if they produce significant interference effects. This thesis aims to address these requirements by presenting the aerodynamic prediction tools, experimental apparatus, and design methodology in order to determine small rotors designs that improve sUAV performance. 


\section{Chapter 3}

\section{Methodology}

There are two aerodynamic models presented in this chapter that are used to estimate the rotor's aerodynamic performance and the flow field of multiple rotors in configuration. The first model consists of a blade element momentum theory-based approach that is formulated for small rotors and is used to estimate the self-induced velocities and the blade loadings of arbitrary rotor geometries. This approach includes sub-routines to model the sectional aerodynamics and several radial inflow formulations both with and without the small angle approximations. The second model consists of a potential flow based approach that uses vortex ring elements to estimate the induced effects of multiple rotors in configuration.

The selection of the appropriate aerodynamic prediction methodologies consisted of evaluating the trade-off between computational expense versus the required amount of detail about the rotor inflow and wake structure. With respect to the model being implemented within a formal optimization approach, a process which requires multitudinous performance evaluations for a wide range of candidate rotor geometries, the forcing function tends to computationally efficiency and model robustness. For these reasons, a blade element theory approach was selected over some of the more complex methods discussed in Sec.2. A comprehensive model validation is also vital for obtaining meaningful performance trends. Though similar models have been validated in hover and axial flight $[19,43,44]$, the predictive capabilities of the performance methodology must be extended to edgewise flight, and is addressed in the work herein. 


\subsection{Vehicle and Rotor Reference Frames}

In addition to the conventional space- and body-fixed reference frames, two additional reference frames are used in this thesis. These are the vehicle reference frame, which is denoted using the subscript $v$ in Fig. 3.1a, and the rotor's local reference frame, shown in Fig. 3.1b. The local reference frame is analogous to the top down view of the rotor's tip path plane and has axes denoted with the subscript TPP. The blade's instantaneous position within the tip path plane is represented by the azimuth angle, $\psi$. The $+x_{\text {TPP }}$ axis for each rotor plane is aligned with the freestream velocity. The rotor angle of attack is equivalent to the vehicle's angle of attack, shown in Fig.3.1a, provided there is no cant or twist of the rotor support arms. When $\alpha=0^{\circ}$, the rotor disk is oriented edgewise towards the freestream, whereas $\alpha=90^{\circ}$ corresponds with the axial, or propeller, working mode.

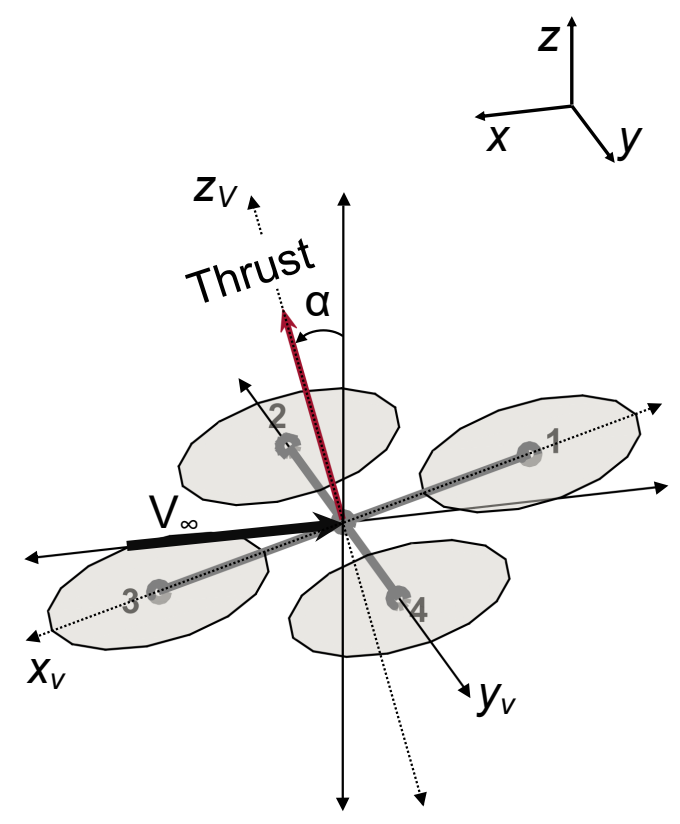

(a) Coordinate systems for the global and vehicle inertial frames

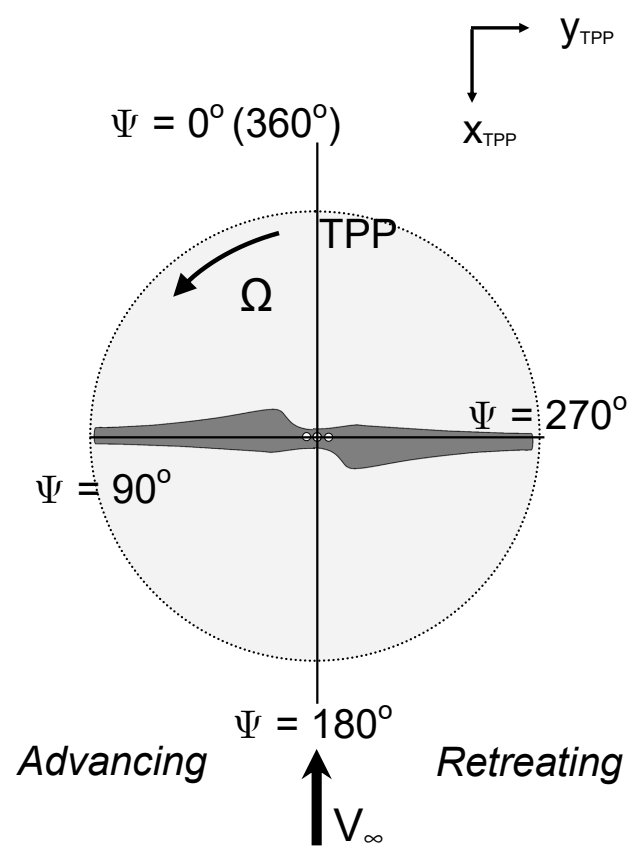

(b) Top view of the tip path plane coordinate system

Figure 3.1: Coordinate systems for the the body-fixed, vehicle and tip path plane reference frames. 


\subsection{Rotor Performance Coefficients}

The forces generated by a rotor or propeller are typically expressed using non-dimensional coefficients so that their performance is characterized independently from their scale and the ambient air density. Rotor and propeller theories differ in their respective definitions of these coefficients and both conventions are shown in Table 3.1. The conversion factors used between rotor and propeller coefficients are derived in Appendix A.1. In the herein presented work, the nomenclature for rotors is used, unless explicitly indicated with the propeller notation subscripts.

\begin{tabular}{|c|c|c|}
\hline Quantity & Rotor Convention (US) & Propeller Convention \\
\hline Thrust & $C_{T}=\frac{T}{\rho A(\Omega R)^{2}}$ & $C_{T, P}=\frac{T}{\rho n^{2} D^{4}}$ \\
Torque & $C_{Q}=\frac{Q}{\rho A \Omega^{2} R^{3}}$ & $C_{Q, P}=\frac{Q}{\rho n^{2} D^{5}}$ \\
Power & $C_{P}=\frac{P}{\rho A(\Omega R)^{3}}$ & $C_{P, P}=\frac{P}{\rho n^{3} D^{5}}$ \\
Longitudinal Force & $C_{F_{x}}=\frac{F_{x}}{\rho A(\Omega R)^{2}}$ & $C_{F_{x}, P}=\frac{F_{x}}{\rho n^{2} D^{4}}$ \\
Lateral Side Force & $C_{F_{y}}=\frac{F_{y}}{\rho A(\Omega R)^{2}}$ & $C_{F_{y}, P}=\frac{F_{y}}{\rho n^{2} D^{4}}$ \\
Rolling moment & $C_{M_{x}}=\frac{M_{x}}{\rho A \Omega^{2} R^{3}}$ & $C_{M_{x}, P}=\frac{M_{x}}{\rho n^{2} D^{5}}$ \\
Pitching moment & $C_{M_{y}}=\frac{M_{y}}{\rho A \Omega^{2} R^{3}}$ & $C_{M_{y}, P}=\frac{M_{x}}{\rho n^{2} D^{5}}$ \\
\hline
\end{tabular}

Table 3.1: Force and moment coefficients common to rotor and propeller theory. 
The ratio of the blade tip speed to its forward velocity is known as the advance ratio. In rotor theory, the advance ratio is decomposed into axial and tangential terms that are defined as:

$$
\begin{gathered}
\mu_{x}=\frac{V_{\infty} \cos \alpha}{\Omega R} \\
\mu_{z}=\frac{V_{\infty} \sin \alpha}{\Omega R}
\end{gathered}
$$

where $\mu_{x}$ represents the freestream velocity parallel the rotor disk and $\mu_{z}$ is perpendicular. A large portion of this thesis consists of comparing the experimental performance rotors oriented at several angles of attack as a function of the total freestream velocity. Thus, the freestream advance ratio, $\mu_{\infty}$, is:

$$
\mu_{\infty}=\frac{V_{\infty}}{\Omega R}
$$

The rotor inflow describes the velocity of the fluid passing through the rotor disk, and is expressed in dimensionless form as the ratio between the sum of the axial velocities and the rotor tip speed, $\Omega R$ :

$$
\lambda=\lambda_{\infty}+\lambda_{i}=\frac{V_{\infty} \sin \alpha+v_{i}}{\Omega R}
$$

where $\lambda_{i}$ and $\lambda_{\infty}$ are the induced and freestream inflow ratios, respectively:

$$
\begin{gathered}
\lambda_{i}=\frac{v_{i}}{\Omega R} \\
\lambda_{\infty}=\frac{V_{\infty} \sin \alpha}{\Omega R}
\end{gathered}
$$




\subsection{Blade Element Momentum Theory Based Approach}

Blade element momentum theory is an approach in which the rotor's induced velocity is found by simultaneously solving blade element and momentum theories with the assumption that they have mutual thrust equivalence. The fundamental equations for this approach are presented in this section, in addition to several methods for modelling the radial inflow distributions in hover and vertical flight. An approach for modelling the inflow in edgewise flight is also included that uses the first harmonic linear inflow model approximation. The methods presented are similar to those used for full scale helicopter rotors, but have been modified specifically for small fixed pitch rotors. This includes the inclusion of the local chord solidity, elimination of some of the small angles approximations, and a method for determining the sectional aerodynamic force coefficients for $\pm 180^{\circ}$ angles of attack.

\subsubsection{Blade Element Theory}

Blade element theory is formulated on the basis that a rotor blade can be represented using a collection of two dimensional elements which are radially distributed along that blade axis. The performance of each element is independent of the adjacent elements and is modelled as though it were a two dimensional airfoil section. Figure 3.2 shows the typical flow environment for the rotor blade, shown in Fig.3.2a, and for a single element, shown in Fig.3.2b. 


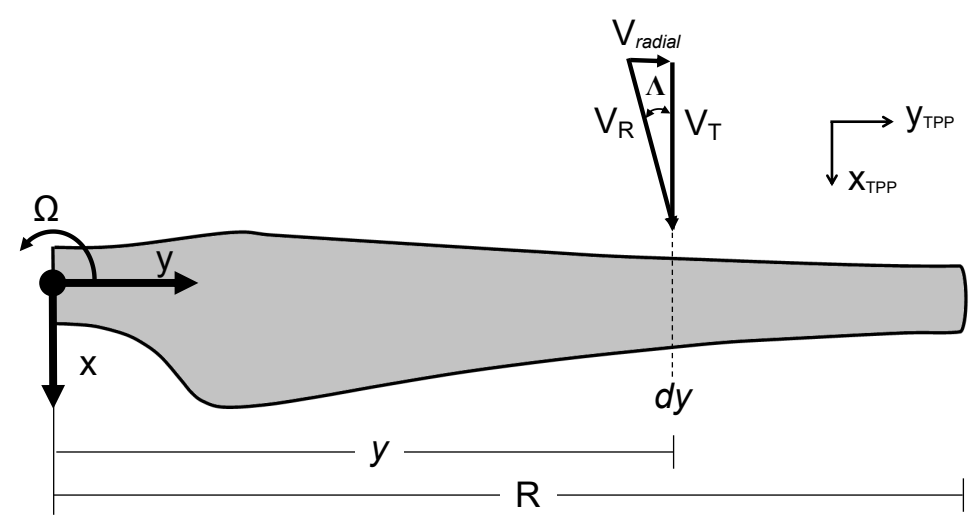

(a) Top view of rotor blade

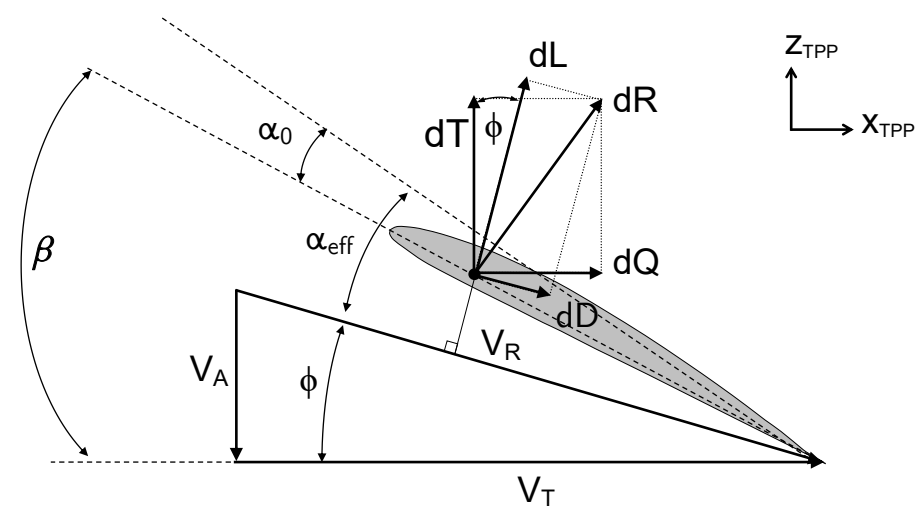

(b) Blade element $d y$ at location $y$

Figure 3.2: Flow environment for the rotor blade and for a typical blade element

Referring to Fig.3.2a, $y$ represents the distance between a blade element and the rotor's rotational axis. The rotational frequency, $\Omega$, is positive counter clock-wise and is measured in radians per second. The sweep angle, $\Lambda$, is the angle between a blade element and the direction of the resultant velocity at the rotor disk and represents the magnitude of the radial velocity, $V_{\text {radial }}$.

In Fig.3.2b the airfoil section is oriented at pitch angle $\beta$ with respect to the rotor's tip path plane. The radial variation in pitch along the blade is its twist distribution, whereas an increase 
or decrease in pitch for the entire blade is called collective pitch. The resultant velocity, $V_{R}$, is the vector sum of the velocity components acting in the $x_{\mathrm{TPP}}$ and $z_{\mathrm{TPP}}$ directions and come from three kinematic sources; the axial and tangential freestream velocity components, $V_{\infty} \sin \alpha$ and $V_{\infty} \cos \alpha$, the section's angular velocity, $\Omega y$, and the induced and swirl velocities, $v_{i}$ and $u_{i}$. In forward flight, the tangential velocity that the element experiences changes in magnitude and direction depending on the blade's instantaneous position and is therefore a function of the azimuth angle, $\psi$. Collecting these terms gives the axial and tangential velocities, $V_{A}$ and $V_{T}$ for each blade element:

$$
\begin{aligned}
& V_{T}=V_{\infty} \cos \alpha \sin \psi+\Omega y-u_{i} \\
& V_{A}=V_{\infty} \sin \alpha+v_{i} \\
& V_{R}=\sqrt{V_{T}^{2}+V_{A}^{2}}
\end{aligned}
$$

The inflow angle, $\phi$, represents the angle between the tip path plane and the resultant velocity and is:

$$
\tan \phi=\frac{V_{T}}{V_{A}}
$$

$\phi$ is quadrant dependant and is therefore taken clockwise when $V_{R}$ is directed downward through the TPP and counter-clockwise when $V_{R}$ is directed upward. The inflow angle is taken over the interval $\{0 \leq|\phi| \leq 180\}$ and thus can be implemented by using the two argument arctangent function.

The effective angle of attack, $\alpha_{\text {eff }}$, is computed by subtracting the inflow angle from the sectional pitch. If the airfoil is cambered, the zero lift angle, $\alpha_{0}$, is added to the effective angle of attack when using thin airfoil theory:

$$
\alpha_{\mathrm{eff}}=\beta(y)+\alpha_{0}-\phi
$$

The differential lift and drag forces, $d L$ and $d D$, and moment, $d M$, are computed provided 
the section's dynamic pressure, chord, and aerodynamic coefficients are known:

$$
\begin{aligned}
d L & =\frac{1}{2} \rho V_{R}^{2} c c_{l} d y \\
d D & =\frac{1}{2} \rho V_{R}^{2} c c_{d} d y \\
d M & =\frac{1}{2} \rho V_{R}^{2} c^{2} c_{m} d y
\end{aligned}
$$

The differential lift, drag and moment are oriented with respect to the section's inflow angle as shown in Fig.3.2b. When the rotor's inflow distribution is not axisymmetric, the differential lift and drag cyclically tilt back and forth about the airfoil's aerodynamic center as a function of the blade's azimuth position. It is therefore useful to resolve the differential terms with respect to TPP plane into components that act in the same direction, independently of the inflow angle. These can then be used to determine the rotor's instantaneous thrust and moment required to counter the reactionary torque. Rearranging Eqs.3.12 and 3.13 gives the differential thrust and torque:

$$
\begin{gathered}
d T=N_{b}(d L \cos \phi-d D \sin \phi) \\
d Q=N_{b}(d L \sin \phi+d D \cos \phi) y
\end{gathered}
$$

where $N_{b}$ is the number of blades. The differential torque is taken with respect to the rotational axis and is therefore multiplied by its radial distance along the blade, $y$. The remaining differential forces and moments, which are the two dimensional elemental forces at different blade azimuth positions, are also found through a transformation to the $x_{\mathrm{TPP}}$ and $z_{\mathrm{TPP}}$ axes. These form the constituents terms in Eqs.3.19-3.23. The blade's power is the amount of work done per unit time, or the torque applied over one rotation, and is proportional to the torque multiplied by the angular velocity, $P=\Omega Q$. The differential power is therefore:

$$
d P=N_{b}(d L \sin \phi+d D \cos \phi) \Omega y
$$

The instantaneous forces and moments over the entire blade are found by integrating their 
respective differential terms along the blade's radius. The cyclical fluctuation in their instantaneous values throughout one full rotation is accounted for by taking the mean of these values as a function of the azimuth position. This requires two integrations, both which can be evaluated using Simpson's rule, and gives the complete steady state forces and moments about the rotor's hub:

$$
\begin{aligned}
F_{\mathrm{Z}_{\mathrm{TPP}}} & =\frac{N_{b}}{2 \pi} \int_{0}^{2 \pi} \int_{R_{\mathrm{hub}}}^{R}(d L \cos \phi-d D \sin \phi) d y d \psi \\
F_{\mathrm{x}_{\mathrm{TPP}}} & =\frac{N_{b}}{2 \pi} \int_{0}^{2 \pi} \int_{R_{\mathrm{hub}}}^{R}(d L \sin \phi+d D \cos \phi) \cos \psi d y d \psi \\
F_{\mathrm{y}_{\mathrm{TPP}}} & =-\frac{N_{b}}{2 \pi} \int_{0}^{2 \pi} \int_{R_{\mathrm{hub}}}^{R}(d L \sin \phi+d D \cos \phi) \sin \psi d y d \psi \\
M_{\mathrm{z}_{\mathrm{TPP}}} & =\frac{N_{b}}{2 \pi} \int_{0}^{2 \pi} \int_{R_{\mathrm{hub}}}^{R}(d L \sin \phi+d D \cos \phi) y d y d \psi \\
M_{\mathrm{x}_{\mathrm{TPP}}} & =\frac{N_{b}}{2 \pi} \int_{0}^{2 \pi} \int_{R_{\mathrm{hub}}}^{R}[(d L \cos \phi-d D \sin \phi) y \cos \psi-d M \sin \psi] d y d \psi \\
M_{\mathrm{y}_{\mathrm{TPP}}}= & \frac{N_{b}}{2 \pi} \int_{0}^{2 \pi} \int_{R_{\mathrm{hub}}}^{R}[(d L \cos \phi-d D \sin \phi) y \sin \psi+d M \cos \psi] d y d \psi
\end{aligned}
$$

where the thrust is $T=F_{\mathrm{Z}}$ and the torque is $Q=M_{\mathrm{z}_{\mathrm{TPP}}}$. The steady state power is then:

$$
P=\frac{N_{b}}{2 \pi} \int_{0}^{2 \pi} \int_{R_{\mathrm{hub}}}^{R}(d L \sin \phi+d D \cos \phi) \Omega y d y d \psi
$$

If the rotor's induced and swirl velocity distributions in Eqs.3.7 and 3.8 are given, Eqs.3.183.25 can be solved directly. Otherwise, $v_{i}$ and $u_{i}$ must be determined iteratively using momentum 
theory and Eq.3.18.

\subsubsection{Momentum Theory}

In momentum theory, the rotor is treated as an infinitesimally thin actuator disk that induces a fluid velocity through it by means of a pressure jump across the disk. If the inflow is assumed to be uniform, the vertical velocity of the fluid gradually increases as it passes through the rotor disk while the velocity throughout any given horizontal cross section within the fluid is constant. Differential momentum theory refines this approach by dividing the disk into a number of concentrically arranged annuli, each having an area given by $d A=2 \pi y d y$. The differential mass flow rate of air through each annulus is given by:

$$
d \dot{m}=\rho\left(V_{A}+v_{i}\right) d A=\rho\left(V_{A}+v_{i}\right) 2 \pi y d y
$$

The thrust of each annulus is proportional to the momentum change of the fluid passing through it, so that $d T=d \dot{m} w$, where $w$ is the slipstream velocity in the far wake. Glauert, in Ref.28, relates the far wake velocity to the velocity at the rotor plane as $v_{i}=\frac{1}{2} w$ and thus, the differential thrust for each annulus is:

$$
d T=4 \pi \rho\left(V_{A}+v_{i}\right) v_{i} y d y
$$

The ideal power can be calculated using first principles and is therefore the product of the thrust and induced velocity at the rotor disk:

$$
d P=d T v_{i}=4 \pi \rho\left(V_{A}+v_{i}\right) v_{i}^{2} y d y
$$

This represents the rotor's minimum attainable induced power because it neglects the viscous effects arising from the the rotor wake and from the motion of the blades. 


\subsubsection{Approaches to Modelling the Rotor Inflow}

Combining Eq.3.15 from blade element theory and Eq.3.27 from momentum theory in essence equates the thrust that is produced by the blade, given by the principal of lift generation by circulation from the Kutta-Joukowski theorem, to the fluid's momentum change through the rotor disk, given by the principles of conservation of mass and momentum. A common simplification is to use small angle approximations in order to simply the radial inflow equation $[4,13,33,45]$. Using this approximation, the sectional aerodynamic coefficients can be estimated using thin airfoil theory or included using tabulated data.

Often fixed pitched rotors are highly twisted, especially if they are designed for minimum induced losses during hover, such as is the case with the hyperbolic distributions suggested by Gessow [29] and Leishman [13]. In these instances, the inboard section lift vector may point more in the in-plane direction and can lead to the under-prediction of power for large inboard solidities. Therefore, another approach, that removes the small angle approximations and includes the in-plane induced velocities, is also included and is based on the methods in Refs.43 and 19.

For edgewise flight, a third approach is presented that uses uniform momentum theory in combination with a first harmonic linear inflow model $[13,28,46]$. This method is similar to the one developed in Ref.13, and is formulated to capture some of the retreating/advancing blade effects and does not use small angle approximations.

\subsubsection{Small Angles Approximations}

The induced velocities can be solved for directly in the hover and vertical flight cases provided that a number of approximations related to a lightly loaded rotor are used. First, the axial component of the velocity is assumed to be much smaller than the tangential velocity. In addition, the in-plane induced velocities are negligible so that $V_{R} \cong V_{T}=\Omega y$ and $u_{i} \cong 0$, respectively. Second, the inflow angle is assumed to be small so that $\phi=\frac{V_{A}}{V_{T}}$ and $\phi \cong \frac{\lambda}{r}$. Third, and also since the inflow angle is small, the section drag force does not significantly contribute to the section thrust force, so that $d D \sin (\phi) \cong 0$ and $d D \cos (\phi) \cong d D$. Applying these assumptions 
to the differential thrust, torque and power in Eqs.3.15-3.17 yields:

$$
\begin{aligned}
& d T=N_{b} d L \\
& d Q=N_{b}(d L \phi+d D) y \\
& d P=N_{b}(d L \phi+d D) \Omega y
\end{aligned}
$$

Using the definitions for the thrust and torque coefficients that are listed in Table 3.1 and the non-dimensional radius, $r=\frac{y}{R}$, the differential thrust and power coefficients with the lightly loaded approximations are:

$$
\begin{aligned}
d C_{T}=\frac{d T}{\rho A(\Omega R)^{2}} & =\frac{N_{b} d L}{\rho \pi R^{2} R^{2} \Omega^{2}} \\
& =\frac{N_{b}\left(\frac{1}{2} \rho(\Omega y)^{2} c c_{l} d y\right)}{\rho \pi R^{4} \Omega^{2}} \\
& =\frac{N_{b} c}{2 \pi}\left(\frac{r}{y}\right) c_{l}\left(\frac{y}{R}\right)^{2} d\left(\frac{y}{R}\right) \\
& =\frac{N_{b} c}{2 \pi y} c_{l} r^{3} d r \\
& =\sigma_{l} c_{l, \alpha}\left(\beta+\alpha_{0}-\phi\right) r^{3} d r \\
& =\sigma_{l} c_{l, \alpha}\left(\left(\beta+\alpha_{0}\right) r-\lambda\right) r^{2} d r
\end{aligned}
$$

and 


$$
\begin{aligned}
d C_{P}=\frac{d P}{\rho A(\Omega R)^{3}} & =\frac{N_{b}(d L \phi+d D) \Omega y}{\rho A(\Omega R)^{3}} \\
& =\frac{N_{b} \frac{1}{2} \rho(\Omega y)^{2} c\left(c_{l} \phi+c_{d}\right) \Omega y d y}{\rho \pi R^{2}(\Omega R)^{3}} \\
& =\frac{N_{b} c}{2 \pi}\left(\frac{r}{y}\right) \frac{y^{3}\left(c_{l} \phi+c_{d}\right)}{R^{3}} d\left(\frac{y}{R}\right) \\
& =\frac{N_{b} c}{2 \pi y} r\left(\frac{y}{R}\right)^{3}\left(c_{l} \phi+c_{d}\right) d r \\
& =\sigma_{l} c_{l} \phi r^{4} d r+\sigma_{l} c_{d} r^{4} d r \\
& =d C_{T} \lambda+\sigma_{l} c_{d} r^{4} d r
\end{aligned}
$$

Equation 3.33 is composed of two terms that represent the induced and profile power, respectively.

The local chord solidity ratio, $\sigma_{l}$, is the ratio between the area of the blade section and the area of the corresponding disk annulus: $\sigma_{l}=\frac{N_{b} c(r)}{2 \pi y}$. For blades with a non-constant chord distributions, the global solidity, $\sigma$, is found by integrating the local chord solidity ratio distribution over the non-dimensional radius:

$$
\sigma(r)=\int_{0}^{1} \frac{N_{b} c(r)}{2 \pi R r} d r
$$

The local chord solidity is related to the global solidity ratio by a factor of $\frac{1}{2 r}$, and so Eq.3.32 reduces to:

$$
d C_{T}=\sigma_{l} c_{l, \alpha} r^{3} d r=\frac{1}{2} \sigma c_{l} r^{2} d r
$$

which is the commonly used form of $C_{T}$ when using the global solidity term to describe blades with rectangular planforms or that have small amounts of taper.

Implementing a tip-loss correction model can estimate some of the three-dimensional induced effects arising from having a finite number of blades with finite span. Prandtl's tip-loss function captures some of these effects and can be easily implemented using momentum theory [20]. Despite tip-loss effects causing a lift reduction near the tips, a more realistic inter- 
pretation of the effect is that the trailing tip vortices in the wake induce in-plane velocities at the blade tips. This increases the a section's induced inflow and, thus, reduces the section's effective angle of attack. Prandtl, who modelled this behaviour using a series of convecting two dimensional vortex sheets, presents a tip-loss factor, $F$, that is given by:

$$
F=\left(\frac{2}{\pi}\right) \cos ^{-1}\left(e^{-\left(f_{\text {tip }} f_{\text {root }}\right)}\right)
$$

where

$$
f_{\text {tip }}=\frac{N_{b}}{2}\left(\frac{1-r}{r \phi}\right)
$$

and

$$
f_{\text {root }}=\frac{N_{b}}{2}\left(\frac{r}{(1-r) \phi}\right)
$$

The tip-loss factor is a function of the number of blades and the section's inflow angle and is included in Eq.3.27:

$$
d T=4 F \pi \rho\left(V_{A}+v_{i}\right) v_{i} y d y
$$

Tip-loss effects decrease with increasing number of blades $(F \rightarrow 1)$ for which the physical interpretation is that as the rotor solidity increases, the inflow approaches the analogue of an actuator disk. This reduces the gradient of the bound circulation approaching the tips and, thus, reduces the strength of their shed vorticity. Practically, a solid disk cannot induce any flow through it, and so minimizing the tip induced effects is a function of tip speed, rotor diameter and number of blades. The tip-loss factor, F, depends on the inflow ratio, hence, must be solved iteratively with the radial inflow distribution. Implementing the tip-loss factor and the inflow ratio into Eq.3.27, the thrust coefficient from momentum theory is written as: 


$$
\begin{aligned}
d C_{T} & =\frac{4 F \pi \rho\left(V_{A}+v_{i}\right) v_{i} y d y}{\rho \pi R^{4} \Omega^{2}} \\
& =4 F\left(\lambda_{\infty}+\lambda_{i}\right) \lambda_{i} r d r \\
& =4 F \lambda\left(\lambda-\lambda_{\infty}\right) r d r
\end{aligned}
$$

Similarly, the induced power coefficient from Eq.3.33 is given by:

$$
d C_{P_{i}}=d C_{T} \lambda=4 F\left(\lambda-\lambda_{\infty}\right) \lambda^{2} r d r
$$

Now that the lightly loaded thrust coefficients from both momentum and blade element theories have been determined, they are equated in order to solve for the radial inflow:

$$
\sigma_{l} c_{l, \alpha}\left(\left(\beta+\alpha_{0}\right) r-\lambda\right) r^{2} d r=4 F\left(\lambda_{\infty}+\lambda_{i}\right) \lambda_{i} r d r
$$

Rearranging for $\lambda$, yields:

$$
\lambda^{2}+\left(\frac{\sigma_{l} c_{l, \alpha}}{4 F} r-\lambda_{\infty}\right) \lambda-\frac{\sigma_{l} c_{l, \alpha}}{4 F}\left(\beta+\alpha_{0}\right) r^{2}=0
$$

which is a quadratic equation for the radial inflow, $\lambda$, and has the solution:

$$
\lambda\left(r, \lambda_{\infty}\right)=\sqrt{\left(\frac{\sigma_{l} c_{l, \alpha}}{8 F} r-\frac{\lambda_{\infty}}{2}\right)^{2}+\frac{\sigma_{l} c_{l, \alpha}}{4 F}\left(\beta+\alpha_{0}\right) r^{2}}-\left(\frac{\sigma_{l} c_{l, \alpha}}{8 F} r-\frac{\lambda_{\infty}}{2}\right)
$$

where $c_{l, \alpha}$ is the section lift-curve slope and from thin airfoil theory is equal to $2 \pi / \mathrm{rad}$. Without the tip-loss factor, Eq.3.44 can be solved directly whereas including it requires an iterative solution process, such as the fixed-point method, which will converge within a few iterations starting with $F_{0}=1$.

To remove the linear lift curve slope assumption, Eqs.3.32 and 3.40 can be used instead and, thus, the inflow equation becomes: 


$$
\begin{gathered}
\sigma_{l} c_{l} r^{3} d r=4 F \lambda\left(\lambda-\lambda_{\infty}\right) r d r \\
\frac{\sigma_{l} c_{l} r^{3} d r}{4 F r d r}=\lambda^{2}-\lambda_{\infty} \lambda \\
0=\lambda^{2}-\lambda_{\infty} \lambda-\frac{\sigma_{l} c_{l} r^{2}}{4 F} \\
\lambda=\frac{\lambda_{\infty}}{2}+\sqrt{\frac{\lambda_{\infty}^{2}}{4}+\frac{\sigma_{l} c_{l} r^{2}}{4 F}}
\end{gathered}
$$

Equation 3.48 is also solved iteratively, but for both the tip-loss factor and the lift coefficient distribution. The section lift coefficient, is found using look-up tables organized by their effective angle of attack and is described in Sec.3.3.8. Equation 3.48 requires considerable more iterations to solve than Eq.3.44. Both forms of the lightly loaded radial inflow equation provide benchmarks for the validation of the large angle inflow approach at a much reduced computational expense.

\subsubsection{Large Angles}

Using the small angle approximations may result in the over-prediction of thrust and the under-prediction of induced power for geometries that have inherently more complicated chord distributions and high inboard pitch angles. Therefore, the following section presents a derivation of the inflow equations which do not use small angle approximations and include the tangential, or swirl, component of the induced velocity.

The differential thrust and power coefficients are again derived from Eqs.3.15 and 3.17, although this time omitting the small angle assumptions: 


$$
\begin{aligned}
d C_{T}=\frac{d T}{\rho A(\Omega R)^{2}} & =\frac{N_{B}(d L \cos \phi-d D \sin \phi)}{\rho \pi R^{2}(\Omega R)^{2}} \\
& =\frac{N_{B}\left(\frac{1}{2} \rho V_{R}^{2} c\right)\left(c_{l} \cos \phi-c_{d} \sin \phi\right)}{\rho \pi R^{2}(\Omega R)^{2}} d y \\
& =\frac{N_{B} c}{2 \pi y}\left(\frac{y}{R}\right) V_{R}^{2}\left(\frac{c_{l} V_{T}-c_{d} V_{A}}{V_{R}(\Omega R)^{2}}\right) d\left(\frac{y}{R}\right) \\
& =\sigma_{l}\left(\frac{y}{R}\right) \sqrt{V_{T}^{2}+V_{A}^{2}}\left(\frac{c_{l} V_{T}-c_{d} V_{A}}{(\Omega R)^{2}}\right) d\left(\frac{y}{R}\right) \\
& =\sigma_{l} \sqrt{\xi^{2}+\lambda^{2}}\left(c_{l} \xi-c_{d} \lambda\right) r d r
\end{aligned}
$$

and

$$
\begin{aligned}
d C_{P}=\frac{d P}{\rho A(\Omega R)^{3}} & =\frac{N_{B}(d L \sin \phi+d D \cos \phi) \Omega y}{\rho \pi R^{2}(\Omega R)^{3}} \\
& =\frac{N_{B}\left(\frac{1}{2} \rho V^{2} c\right)\left(c_{l} \sin \phi+c_{d} \cos \phi\right) \Omega y}{\rho \pi R^{2}(\Omega R)^{3}} d y \\
& =\frac{N_{B} c}{2 \pi y}\left(\frac{y}{R^{2}}\right) V^{2}\left(\frac{c_{l} V_{T}+c_{d} V_{A}}{V(\Omega R)^{2}}\right) y d\left(\frac{y}{R}\right) \\
& =\sigma_{l} \sqrt{V_{T}^{2}+V_{A}^{2}}\left(\frac{c_{l} V_{T}+c_{d} V_{A}}{(\Omega R)^{2}}\right)\left(\frac{y}{R}\right)^{2} d\left(\frac{y}{R}\right) \\
& =\sigma_{l} \sqrt{\xi^{2}+\lambda^{2}}\left(c_{l} \xi-c_{d} \lambda\right) r^{2} d r
\end{aligned}
$$

where $\sin \phi=\frac{V_{A}}{V_{R}}$ and $\cos \phi=\frac{V_{T}}{V_{R}}$. The swirl ratio, $\xi$, is defined as:

$$
\xi=\frac{V_{T}}{\Omega R}=\frac{\Omega y-u_{i}}{\Omega R}
$$

The differential thrust and power coefficients from momentum theory, Eqs.3.40 and 3.41, can be re-arranged to include the swirl and inflow ratios and are:

$$
d C_{T}=4|\lambda| \lambda_{i} r d r
$$




$$
d C_{P}=4|\lambda| \xi_{i} r^{2} d r
$$

where the induced swirl ratio is:

$$
\xi_{i}=\frac{u_{i}}{(\Omega R)}
$$

A tip-loss factor is again implemented into the momentum equations, but this time is extended to large angles, based on the method in Ref.43. As mentioned earlier, the mechanism by which the tip-loss factor affects the radial loading is through an increase in the local inflow at the tip and, thus, for the large angles approach, tip-effects are modelled using both the inflow and swirl ratio terms. The Prandtl tip-loss factor is formulated on the basis that the tip vortices contribute to the induced velocity components as though they act normal to their respective force vectors. For example, for $d C_{T}$, the tip-loss factor, is only completely representative when the inflow angle is zero, $\phi=0$, so that the tip vorticity is inducing velocity in the vertical direction only. When $\phi=90$, however, the tip vorticity induces in-plane swirl velocities and has no effect on the vertical velocities. In other words, the tip-loss factors must approach to zero as the induced angle becomes parallel to the respective force vector. Therefore, the factors $K_{T}$ and $K_{P}$, which represent the thrust and power tip-loss factors, respectively, are introduced to capture this behaviour:

$$
\begin{gathered}
d C_{T}=4 K_{T}|\lambda| \lambda_{i} r d r \\
d C_{P}=4 K_{P}|\lambda| \xi_{i} r^{2} d r
\end{gathered}
$$

where $K_{T}=[1-(1-F) \cos \phi]$ and $K_{P}=[1-(1-F) \sin \phi]$. The inflow angle is the arctangent of the inflow and swirl velocities:

$$
\tan \phi=\frac{\lambda}{\xi}
$$


Equating $d C_{T}$ and $d C_{P}$ for large angle blade element theory and momentum theory gives following system of equations with two unknowns, $\lambda$ and $\xi$ :

$$
\begin{gathered}
\sigma \sqrt{\xi^{2}+\lambda^{2}}\left(c_{l} \xi-c_{d} \lambda\right) r d r=4 K_{T}|\lambda| \lambda_{i} r d r \\
\sigma \sqrt{\xi^{2}+\lambda^{2}}\left(c_{l} \xi-c_{d} \lambda\right) r^{2} d r=4 K_{P}|\lambda| \xi_{i} r^{2} d r
\end{gathered}
$$

In order to find a real solution to the inflow and swirl components, the system must be expressed in the form of $F(x)=0$, for which a numerical optimization approach can be used to find a solution. The induced inflow, $\lambda_{i}=\lambda-\lambda_{\infty}$, and induced swirl ratio, $\xi_{i}=r-\xi$, are also substituted into Eqs.3.58 and 3.59 and give:

$$
\begin{gathered}
\sigma \sqrt{\xi^{2}+\lambda^{2}}\left(c_{l} \xi-c_{d} \lambda\right) d r-4 K_{T}|\lambda|\left(\lambda-\lambda_{\infty}\right) d r=0 \\
\sigma \sqrt{\xi^{2}+\lambda^{2}}\left(c_{l} \xi-c_{d} \lambda\right) d r-4 K_{P}|\lambda|(r-\xi) d r=0
\end{gathered}
$$

To find $\lambda$ and $\xi$ for each radial station, Eqs. 3.60 and 3.61 are solved using a non-linear system solver, such as the trust-region reflective algorithm implemented using the MATLAB ${ }^{\text {TM }}$ fsolve function [47]. Trust region methods are effective for solving unconstrained minimization problems and work by approximating the objective function, $F(x)$, with a simpler function that is representative within a specified area of interest (i.e. a trust region).

\subsubsection{Forward Flight}

In forward flight, the rotor must provide a thrusting force to overcome the vehicle's drag and a lift force equivalent to its weight. This requires the rotor plane to be tilted forward at an angle of attack, $\alpha$. As a consequence, the freestream velocity has a component tangential to the rotor plane, $V_{T}$, as described in Eq.3.7. The subsequent total velocity, $V_{R}$, at the rotor disc is then:

$$
V_{R}=\sqrt{\left(V_{\infty} \cos \alpha\right)^{2}+\left(V_{\infty} \sin \alpha+v_{i}\right)^{2}}
$$

Using uniform momentum theory, the thrust is: 


$$
T=2 \rho A V_{R} v_{i}=2 \rho A v_{i} \sqrt{\left(V_{\infty} \cos \alpha\right)^{2}+\left(V_{\infty} \sin \alpha+v_{i}\right)^{2}}
$$

According to momentum theory the induced velocity under static (hover) condition is given by:

$$
v_{h}=\sqrt{\frac{T}{2 \rho A}}
$$

Substituting thrust in Eq.3.63 with Eq.3.64, the induced velocity during forward flight, $v_{i}$, can be expressed as:

$$
v_{i}=\frac{v_{h}^{2}}{\sqrt{\left(V_{\infty} \cos \alpha\right)^{2}+\left(V_{\infty} \sin \alpha+v_{i}\right)^{2}}}
$$

Using the definitions for the inflow ratio and the advance ratio parallel to the rotor disk, Eqs.3.1 and 3.4 respectively, Eq.3.65 can be re-arranged and used to determine the induced inflow ratio:

$$
\lambda_{i}=\frac{\lambda_{h}^{2}}{\sqrt{\mu_{x}^{2}+\lambda^{2}}}
$$

The hover inflow ratio, $\lambda_{h}$, assuming a constant disk loading is $\lambda_{h}=\sqrt{\frac{C_{T}}{2}}$. So, Eq.3.66 becomes:

$$
\lambda_{i}=\frac{C_{T}}{2 \sqrt{\mu_{x}^{2}+\lambda^{2}}}
$$

Equation 3.63 reduces to the static (hover) case when $V_{\infty}=0$, and with $v_{i}=v_{h}$ and when the freestream inflow component is much larger than the induced inflow, it becomes Glauert's high speed approximation:

$$
\begin{aligned}
T & =2 \rho A v_{i} \sqrt{\left(V_{\infty} \cos \alpha\right)^{2}+\left(V_{\infty} \sin \alpha+v_{i}\right)^{2}} \\
& =2 \rho A v_{i} \sqrt{V_{\infty}^{2}+2 V_{\infty} v_{i} \sin \alpha+v_{i}^{2}} \\
& =2 \rho A V_{\infty} v_{h}
\end{aligned}
$$


Glauert proposed that the thrust of a rotor in high speed forward flight will produce the lift of an elliptically loaded wing of circular planform [28]. The high speed approximation relates this downwash to the rotors inflow as a function of advance ratio and thrust coefficient. For advance ratios exceeding $\mu_{x}=0.1$, Glauert's approximation that $\mu_{x}>\lambda$ fits the result derived from momentum theory and so Eq.3.67 becomes:

$$
\lambda_{i}=\frac{C_{T}}{2 \mu_{x}}
$$

To inflow ratio in Eq.3.4 is a combination of the induced and freestream inflow ratios and, therefore, are re-arranged to include the advance ratio parallel to the rotor, $\mu_{x}$, and the induced inflow ratio, in Eq. 3.67, so that:

$$
\begin{aligned}
\lambda=\frac{V_{A}+v_{i}}{\Omega R} & =\frac{V_{\infty} \sin \alpha}{\Omega R}+\frac{v_{i}}{\Omega R} \\
& =\mu \tan \alpha+\lambda_{i}
\end{aligned}
$$

Grouping the freestream and induced terms from Eqs.3.70 and 3.67 culminates in the final form of the forward flight inflow ratio which is:

$$
\lambda=\mu \tan \alpha+\frac{C_{T}}{2 \sqrt{\mu^{2}+\lambda^{2}}}
$$

Finding a solution to Eq.3.71 requires an iterative solution process. This is expidated by using the Newton-Raphson iteration method, for example as shown by Leishman [13]. This method makes use of a function's first derivative to determine its local gradient and, thus, over a number of iterations, converges on a minima. The Newton-Raphson convergence scheme is described as:

$$
\lambda_{n+1}=\lambda_{n}-\left[\frac{f(\lambda)}{f^{\prime}(\lambda)}\right]_{n}
$$

where $n$ is the iteration number. Re-arranging Eq. 3.71, into the form of $f(\lambda)=0$ gives: 


$$
f(\lambda)=\lambda-\mu \tan \alpha-\frac{C_{T}}{2 \sqrt{\mu^{2}+\lambda^{2}}}=0
$$

and differentiating with respect to $\lambda$ :

$$
f^{\prime}(\lambda)=1+\frac{C_{T}}{2}\left(\mu^{2}+\lambda^{2}\right)^{-3 / 2} \lambda
$$

The hover inflow ratio can be taken as an initial point, $\lambda_{0}=\lambda_{h}$, and the initial $C_{T}$ is calculated from blade element theory with $v_{i, 0}=0$. With the exception of cases where momentum theory is violated, the $\lambda_{h}$ starting point converges to a real solution for most angles of attack and forward flight speeds that are within a reasonable operating range, including the windmill brake state. Convergence is found when the difference between successive iterations, $\epsilon$, for both $\lambda$ and $C_{T}$ is less than: $\epsilon \leq 0.0005$, which is also suggested in Ref.13.

Foreseeable instances for which the solution does not converge and that are within the conceivable operating regime for a small sUAV multicopter, include some axial descent rates (i.e. entering into the vortex ring state) and the high negative rotor plane angles of attack experienced during an air braking manoeuvre. In both instances, the freestream component perpendicular to the rotor disc is small compared to the induced velocities, $V_{\infty} \sin \alpha<2 v_{i}$, and, thus, momentum theory is invalid.

For fixed blades, the inboard portion of the blade is difficult to model using momentum theory, even with the assumption that the flow in this region is strictly two dimensional. There are two cases for which convergence issues exist: when a blade section successively iterates between the normal working state and stall, and when multiple numerical solutions for the inflow are possible. Some of these issues are discussed for wind turbines in Refs.48 and 49. To ensure convergence of the present methodology, a maximum number of iterations are permitted for determining the inflow for the inboard portion of the blade. If the convergence limit is exceeded, an intermediate inflow ratio selected. Despite cases where this assumption may be unrepresentative of the section's physical inflow, the resultant velocities on the inboard blade are small and, therefore, their contribution to the overall thrust and torque is small. 


\subsubsection{Linear Inflow Models}

In forward flight, the rotor wake is swept backwards and is strongly dominated by the presence of tip vortices. Thus, the assumption of uniform inflow is no longer valid. In reality, the wake structure is highly complicated due to the interactions between the wake elements, in particular in the tip region, and with tip vortices interacting with the following passing blades. Modelling these interactions for a specific operating condition requires the use of more sophisticated methods and comes at much greater computational expense [50]. Therefore a number of linear inflow models are available which can estimate the azimuthal induced velocity distributions without having to explicitly model the wake.

The angle at which the rotor is swept back is known as the skew angle, $\chi$. The skew angle is a function of advance ratio and the thrust loading and increases with advance ratio ${ }^{1}$ :

$$
\tan \chi=\left(\frac{\mu_{x}}{\mu_{z}+\lambda_{i}}\right)
$$

Glauert suggested that the resulting inflow at the rotor disk can be represented using a first harmonic non-uniform model that varies longitudinally [28]. This model has since been extended to also include the lateral inflow variation as well:

$$
\lambda_{i}=\lambda_{0}\left(1+k_{x} r \cos \psi+k_{y} r \sin \psi\right)
$$

where $k_{x}$ and $k_{y}$ are the longitudinal and lateral weighing factors. $\lambda_{0}$ is the induced inflow ratio from uniform momentum theory. Attempts have been made using experimentation and vortex wake theories to determine the magnitude of these scaling factors such as in Refs.51-54. According to Refs.13 and 46, the nonuniform inflow models developed by Drees [52] and Pitt and Peters [54] agree best with experimental observations compared to most other models. These models are summarized in Table 3.2.

\footnotetext{
${ }^{1} \mathrm{~A}$ depiction of the skew angle is shown in Sec.3.4 in Fig.3.7b.
} 


\begin{tabular}{|c|c|c|}
\hline Model & $\boldsymbol{k}_{\boldsymbol{x}}$ & $\boldsymbol{k}_{\boldsymbol{y}}$ \\
\hline Drees [52] & $\frac{4}{3}\left(1-\cos \chi-1.8 \mu^{2}\right) / \sin \chi$ & $-2 \mu$ \\
\hline Pitt \& Peters [54] & $\frac{15 \pi}{23} \tan (\chi / 2)$ & 0 \\
\hline
\end{tabular}

Table 3.2: Estimates of $k_{x}$ and $k_{y}$ for the first harmonic inflow distribution models.

Although these models do not explicitly model the tip effects, the loss lift is often approximated by applying a slight reduction factor to the rotor disk's area [55]. Some of this behaviour can be approximated when summing the blade forces using Simpson's rule by imposing a zero thrust loading condition at the tip.

\subsubsection{Blade Section Aerodynamic Characteristics}

A robust method is needed to determine a section's aerodynamic characteristics. This includes the representation of blades sections operating at low Reynolds numbers and at angles of attack $\pm 180^{\circ}$. Although the flow field around the inboard retreating sections is significantly three dimensional, and thus difficult to model with a blade element approach, the sectional coefficients can provide an approximate estimate of the profile drag. The approach that was used in the herein presented research consists of a table-lookup scheme that is coupled with a post stall model.

The tables consist of sectional aerodynamic coefficients that were generated using experimental results [17], or predictions methods, such as XFOIL [56]. The coefficients are grouped by Reynolds number and sorted by angle of attack. The lift, drag and moment coefficients for an input angle of attack are found by cubic interpolation and are output along with an indication of whether they are below or above $c_{l, \max }$ or $c_{l, \min }$, respectively. If data is supplied for the stalled range, the lookup scheme defaults to using the tabulated data opposed to reverting to the stall model.

An example of the lift, drag and moment coefficients, for a representative sUAV rotor blade section airfoil are shown in Figs.3.3 through 3.5. Figure 3.3 shows that for several angles of attack, the lift coefficient deviates from the result predicted using a linear lift curve slope, which is indicated by the red line. This is also important when determining a section's drag coefficient, 
which when modelled using other approaches, such as a polynomial or canonical representation, may result in an under-prediction. For example, this is shown in Figs.3.4 and 3.5, which show outlier points of $c_{d}$ and $c_{m}$ for the $\operatorname{Re}=50,000$ curve due to a laminar separation bubble.

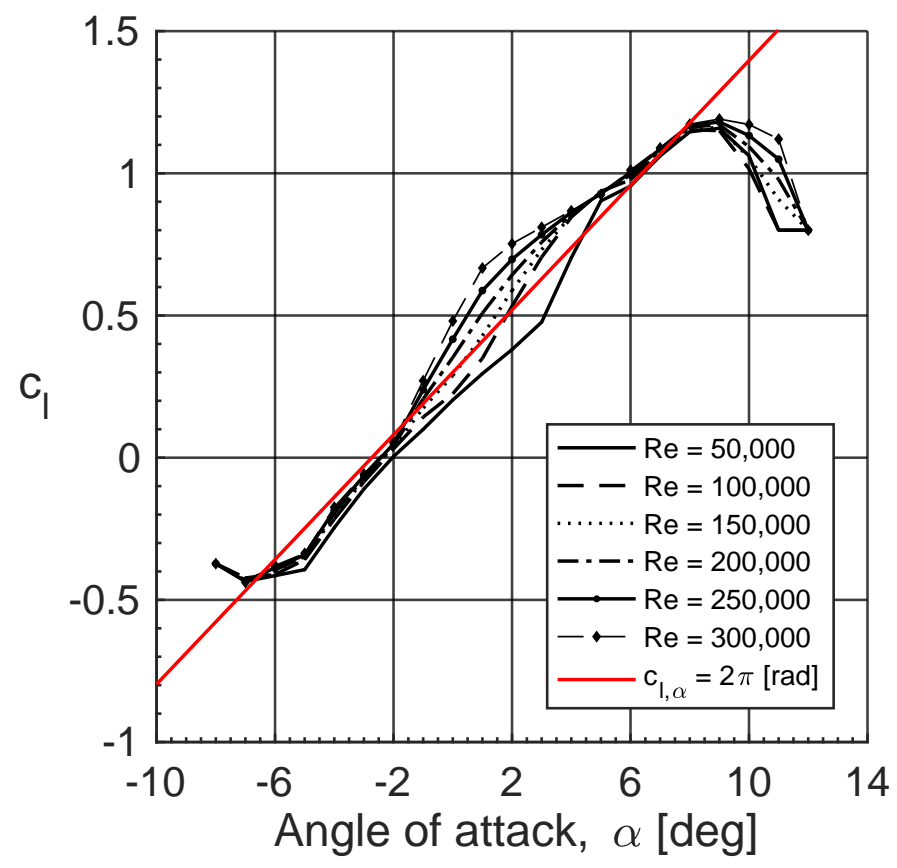

Figure 3.3: Lift coefficient vs. angle of attack for the MA409 airfoil 


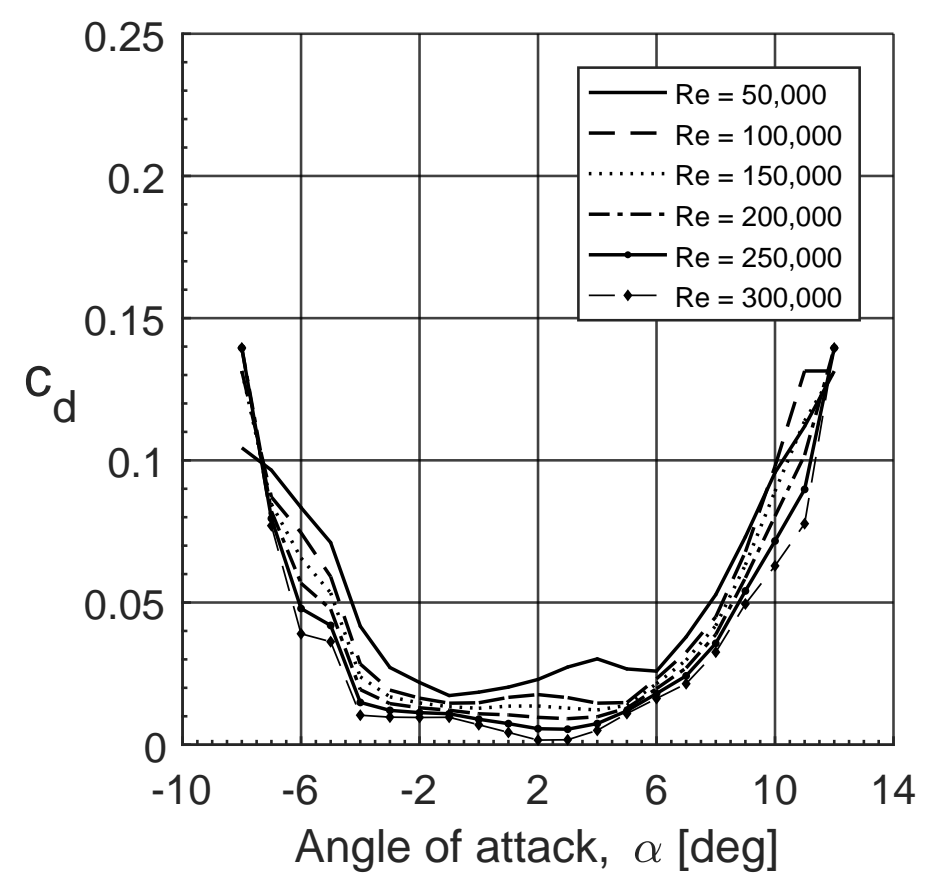

Figure 3.4: Drag coefficient vs. angle of attack for the MA409 airfoil

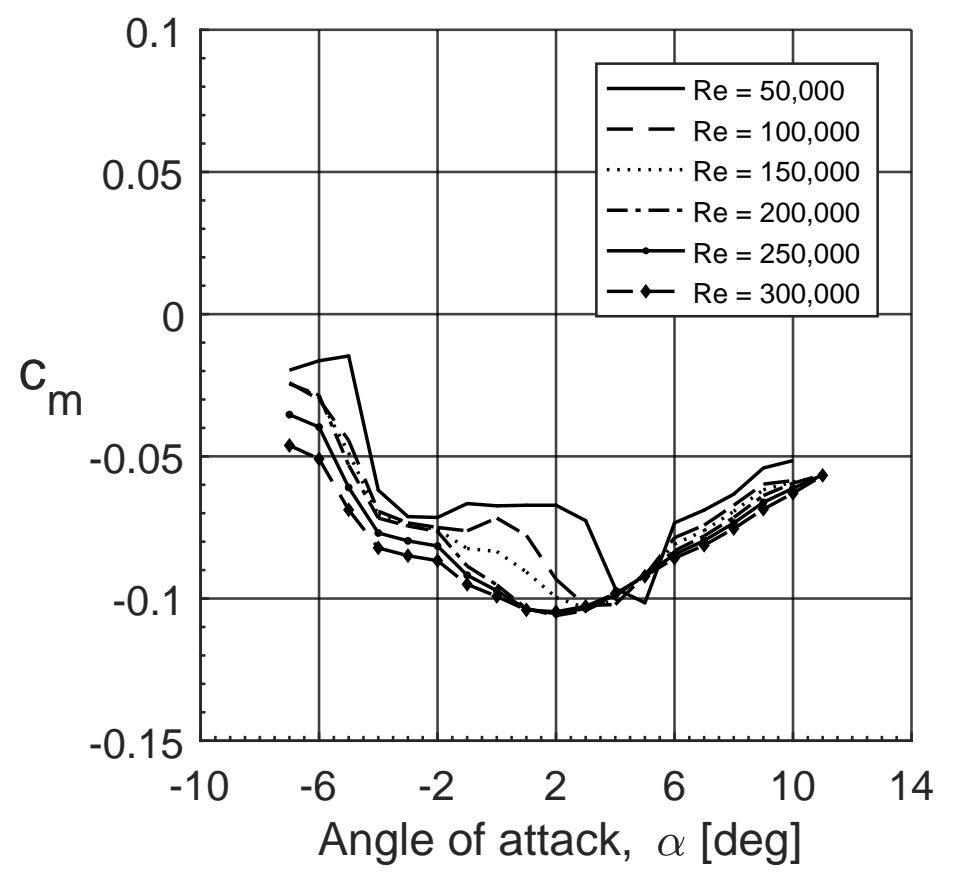

Figure 3.5: Moment coefficient vs. angle of attack for the MA409 airfoil 
Much of wind tunnel data used is measured for Mach numbers below the compressible range and therefore compressibility effects are neglected. As shown in Sec.5.2.3, the blade tips on the largest diameter rotor tested do not operate close to the drag divergence range, even for large advance ratios. Effects that dynamic stall, unsteadiness in the wake and radially induced swirl velocities have on the sectional performance are neglected.

A section's aerodynamic coefficients in stall are estimated using a series of empirical equations in Refs.57 and 58 that were developed from experimental data for wind turbine blades. Reference 44 demonstrated that this model could be applied to small propellers. The equations are based on the assumption that the flow is fully separated on one side of the airfoil so that, for airfoils of relatively small thickness, their lift and drag coefficients approach those of a flat plate as the effective angle of attack approaches $90^{\circ}$. Lift, drag, and moment coefficients over the entire range are plotted in Fig.3.6. The normal, tangential, lift, drag and moment coefficients are computed using the following relationships:

$$
\begin{aligned}
c_{n} & =c_{d, 90} \frac{\sin \alpha_{\mathrm{eff}}}{0.56+0.44 \sin \alpha_{\mathrm{eff}}} \\
c_{t} & =\frac{c_{d, 0} \cos \alpha_{\mathrm{eff}}}{2} \\
c_{l} & =c_{n} \cos \alpha_{\mathrm{eff}}-c_{t} \sin \alpha_{\mathrm{eff}} \\
c_{d} & =c_{n} \sin \alpha_{\mathrm{eff}}+c_{t} \cos \alpha_{\mathrm{eff}} \\
c_{m} & =-c_{n}\left[0.25-0.175\left(1-\frac{2 \alpha_{\mathrm{eff}}}{\pi}\right)\right]
\end{aligned}
$$

where $c_{n}$ and $c_{t}$ are the normal and tangential force coefficients, respectively, and $c_{d, 0}$ is the drag coefficient at $\alpha=0$. The coefficients above are taken from Ref.57, with the exception of the normal force coefficient, $c_{n}$, which is from Ref.59. The lift, drag and moment coefficients for angles of attack $\pm 180^{\circ}$ are shown in Fig.3.6.

Despite the aerodynamic force coefficients for the stalled region agreeing with the flat plate result at $\alpha=90^{\circ}$, there are a number of limitations to the model. For example, for some airfoils, there exists a discontinuity between the table look-up data and the stall model data for angles 
of attack just beyond the limits of the table data. This results in a shift between the coefficient values for consecutive angles of attack, where the stall model under-predicts the coefficient. To help blend the two, the coefficient at the endpoint from the table data is used until the stall model predicts a value exceeding this and corresponding to a larger angle of attack. This is shown in Fig.3.6 for the lift coefficient, between 10.5 to 22 degrees, and for the drag coefficient, between -8 to -12 degrees, which have transition areas where the coefficients are uniform. The second limitation of the model that some of the discrete features of the airfoil performance are not represented, such as taking into account the shifting of the aerodynamic center, and representing stall when the airfoil's trailing edge is pointed towards the freestream. Despite these limitations, this approach for representing the sectional aerodynamic coefficients in stall is acceptable, especially considering the coefficients for $\pm 180^{\circ}$ are represented. 

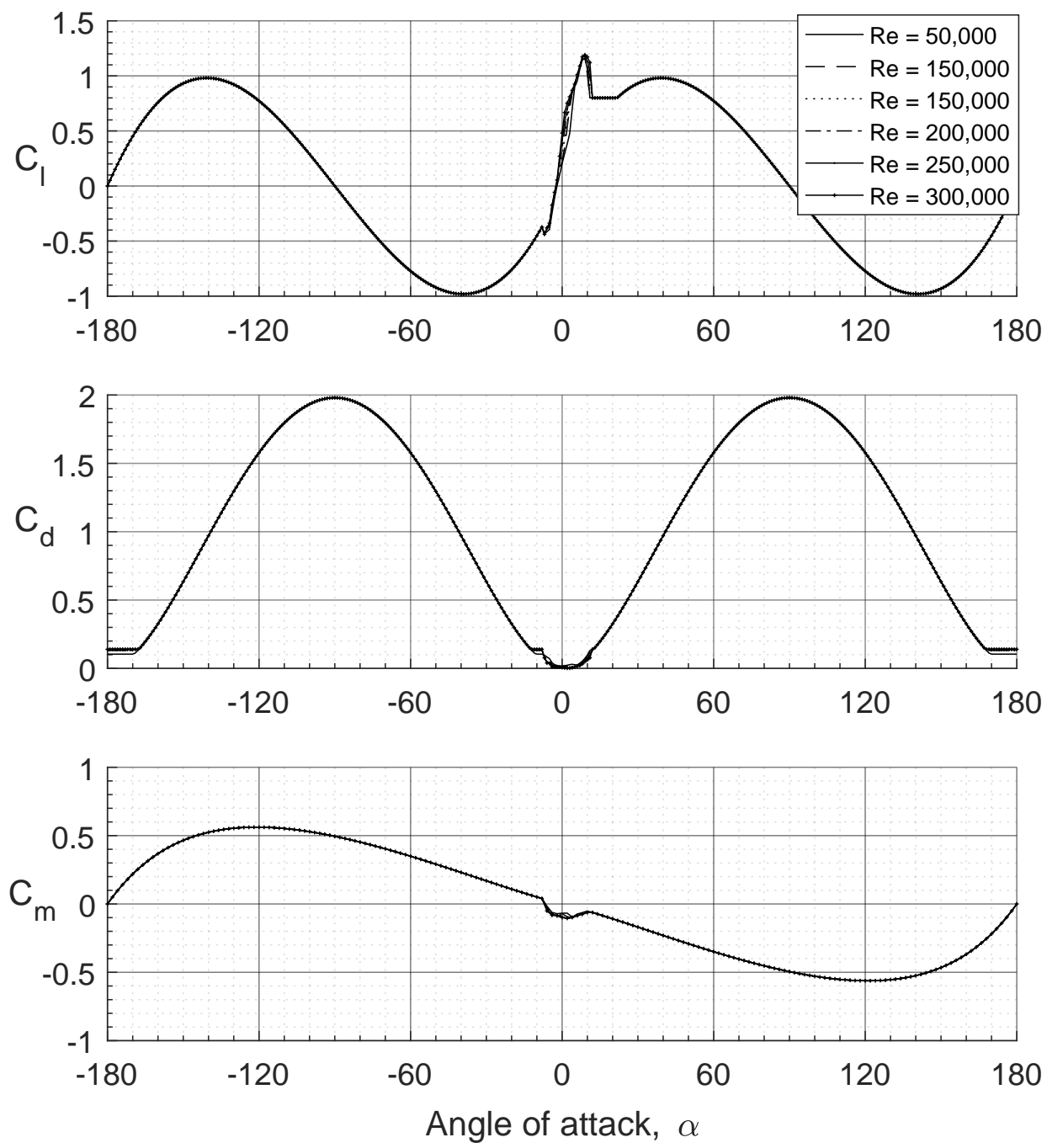

Figure 3.6: Sectional lift, drag and moment coefficients for the MA409 for a $\pm 180^{\circ}$ angle of attack sweep

\subsection{Vortex Wake Interaction Model}

The aerodynamic performance of multirotor sUAV s differ from conventional helicopters due to the wake interaction of adjacently positioned rotors. The choice of rotor configuration has an effect on the rotor's inflow distribution and typically results in an increase in induced power. One approach to determining the wake geometry is to use a potential flow based approach, where 
the rotor's tip vortices are modelled as series of discretized vortex line segments in the form of a stacked rings propagating downwards.

A similar wake interference model is presented in Ref. [60] that is implemented on a vehicle performance level. The difference between these models is that the aim of this approach is to model effect that adjacent rotors have on rotor's inflow distribution.

A wake rigid wake structure that is pre-defined and does not contract downstream is known as a prescribed fixed wake. The justification of using such a model is based on the following assumptions:

1. The rotor is lightly loaded and can be approximated by a fixed wake with negligible contraction.

2. The strength of the blade's bound circulation assumes it has constant induced velocity across the disk. This assumption largely holds for lightly loaded rotors that have near-ideal loadings.

3. The interaction between adjacent vortex wake elements and between the rotor blade and vortex elements are neglected.

4. Unsteadiness in the wake and lifting blade is neglected.

5. Viscous forces in the wake are negligible.

Using these assumptions, the circulation, $\Gamma$, of a shed tip vortex is calculated from the rotor's thrust, T, using:

$$
\Gamma=\frac{2 T}{N_{B} \rho \Omega R^{2}}
$$

In hover, the wake propagates vertically downward at a rate proportional to the far field induced velocity, which is assumed to be proportional to its inflow $\left(\lambda_{h}=\sqrt{C_{T} / 2}\right)$. In forward flight, the wake's direction is dependant on both the thrust loading and rotor's angle of attack 
and propagates at a skew angle, $\chi$. If it is assumed that $\lambda_{i} \approx \lambda_{h}$ at an equivalent thrust loading, then the skew angle is re-arranged to be:

$$
\chi=\tan ^{-1}\left(\frac{V_{\infty} \cos \alpha}{V_{\infty} \sin \alpha+\sqrt{\frac{T}{2 \rho A}}}\right)
$$

A wake element refers to a ring wake element formed for one full rotation of a single blade. A wake segment refers to the straight line segments that together form a ring wake element. The wake structure for a single rotor is therefore the collection of stacked ring wake elements. The vertical spacing between these ring elements depends on the disk loading, rotor's rotational speed, number of blades and the skew angle, and is given by:

$$
z_{v_{i}}=\frac{2 \pi}{N_{B} \Omega}\left(\sqrt{\frac{T}{2 \rho A}}+V_{\infty} \sin \alpha\right)
$$

where $z_{v_{i}}$ is oriented perpendicular to the rotor disk reference frame. The horizontal spacing is implicitly solved from the skew angle and vertical spacing.

\subsubsection{Wake Induced Velocities}

Evaluating the velocities induced by the wake structure on the rotor plane consists of an iterative routine that individually calculates the effect that individual vortex segments have on collocation points place strategically along the rotor disk. These collocation points are distributed at locations coinciding with the position of the blade elements.

The velocities induced at a collocation point, $\mathrm{P}$, by a single vortex segment is determined using the Biot-Savart law, and is shown in Fig.3.7a. Ref.61 presents a number of equations for finding this induced velocity, $v_{i, \text { seg }}$, given by:

$$
v_{i, \mathrm{seg}}=\frac{r_{1} \times r_{2}}{\left|r_{1} \times r_{2}\right|} \frac{\Gamma}{4 \pi d} \cdot\left(\cos \beta_{1}-\cos \beta_{2}\right)
$$

where

$$
d=\frac{\left|r_{1} \times r_{2}\right|}{\left|r_{0}\right|}
$$


and

$$
r_{0}=r_{1}-r_{2}
$$

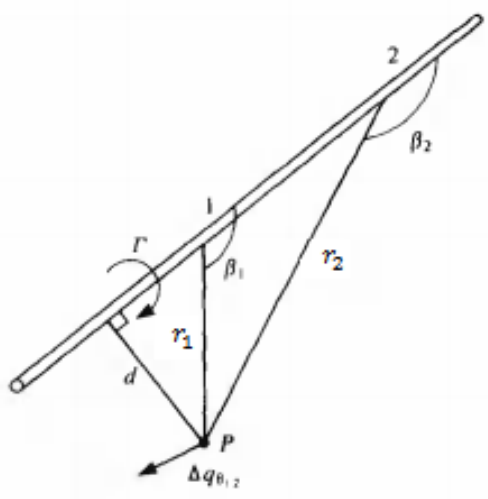

(a) Example of a vortex segment's induced effect on point [61].

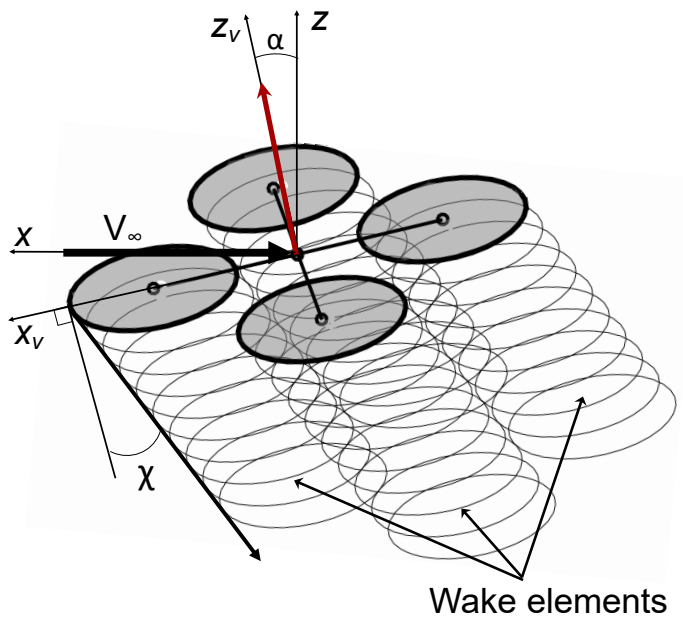

(b) Geometry of skewed wake structure.

Figure 3.7: Vortex segment and wake geometry

Often multirotors can incorporate support arm twist or cant into their design for more yaw authority or to induce a dihedral effect, respectively. A positive twist angle is defined as a counter clockwise rotation about $y$-axis on the rotor plane coordinate system whereas cant is defined as a counter clockwise rotation of the entire rotor disk about an orthogonal axis on the vehicle's $x y$ plane and passing through vehicle's center point. In these instances, the vertical induced velocity component must be transformed from the vehicle reference frame to the rotor reference frame for each individual rotor. The matrices below represent the transformations between coordinate systems where the matrices Eqs.3.90 and 3.91 represent the twist and cant rotation matrices from the rotor to vehicle coordinate systems. Included also are the transformations matrices from the global-fixed to vehicle-fixed reference frames for when the vehicle is collectively pitched and or rolled, respectively, using Eqs.3.90 and 3.91. 


$$
\begin{array}{ccc}
\mathbf{T}_{\mathrm{twist}} & =\left[\begin{array}{ccc}
1 & 0 & 0 \\
0 & \cos \varepsilon & \sin \varepsilon \\
0 & -\sin \varepsilon & \cos \varepsilon
\end{array}\right] \quad(3.88) & \mathbf{T}_{\text {cant }}=\left[\begin{array}{ccc}
\cos \gamma & 0 & -\sin \gamma \\
0 & 1 & 0 \\
-\sin \gamma & 0 & \cos \gamma
\end{array}\right] \\
\mathbf{T}_{\text {pitch }}=\left[\begin{array}{ccc}
\cos \alpha & 0 & -\sin \alpha \\
0 & 1 & 0 \\
\sin \alpha & 0 & \cos \alpha
\end{array}\right] \quad(3.90) & \mathbf{T}_{\text {roll }}=\left[\begin{array}{ccc}
1 & 0 & 0 \\
0 & \cos \Phi & -\sin \Phi \\
0 & \sin \Phi & \cos \Phi
\end{array}\right]
\end{array}
$$

where $\gamma$ and $\varepsilon$ are the cant and twist angles relative to the vehicles reference frame and $\alpha$ and $\Phi$ are the vehicle's pitch and roll angles relative to the global reference frame.

These matrices can also be used to transform the rotor forces and moments in Eqs.3.19 through 3.23 between the rotor and vehicle reference frames.

\subsection{Implementation}

\subsubsection{Performance Prediction Program}

The aerodynamic methodologies presented in the previous sections were implemented into a rotor performance prediction program. For single rotor performance, the program is organized similar to a conventional blade element momentum approach and the general algorithm is shown in Fig.3.8. Two of the three inflow models require explicit convergence routines to determine the inflow distribution, and are represented by the shaded boarders. The approach used for edgewise flight also requires an additional nested iteration scheme between the rotor's thrust and the inflow variation in order to incorporate the linear inflow model. The third uses MATLAB's built in non-linear equation solver function and is performed for each blade station. 


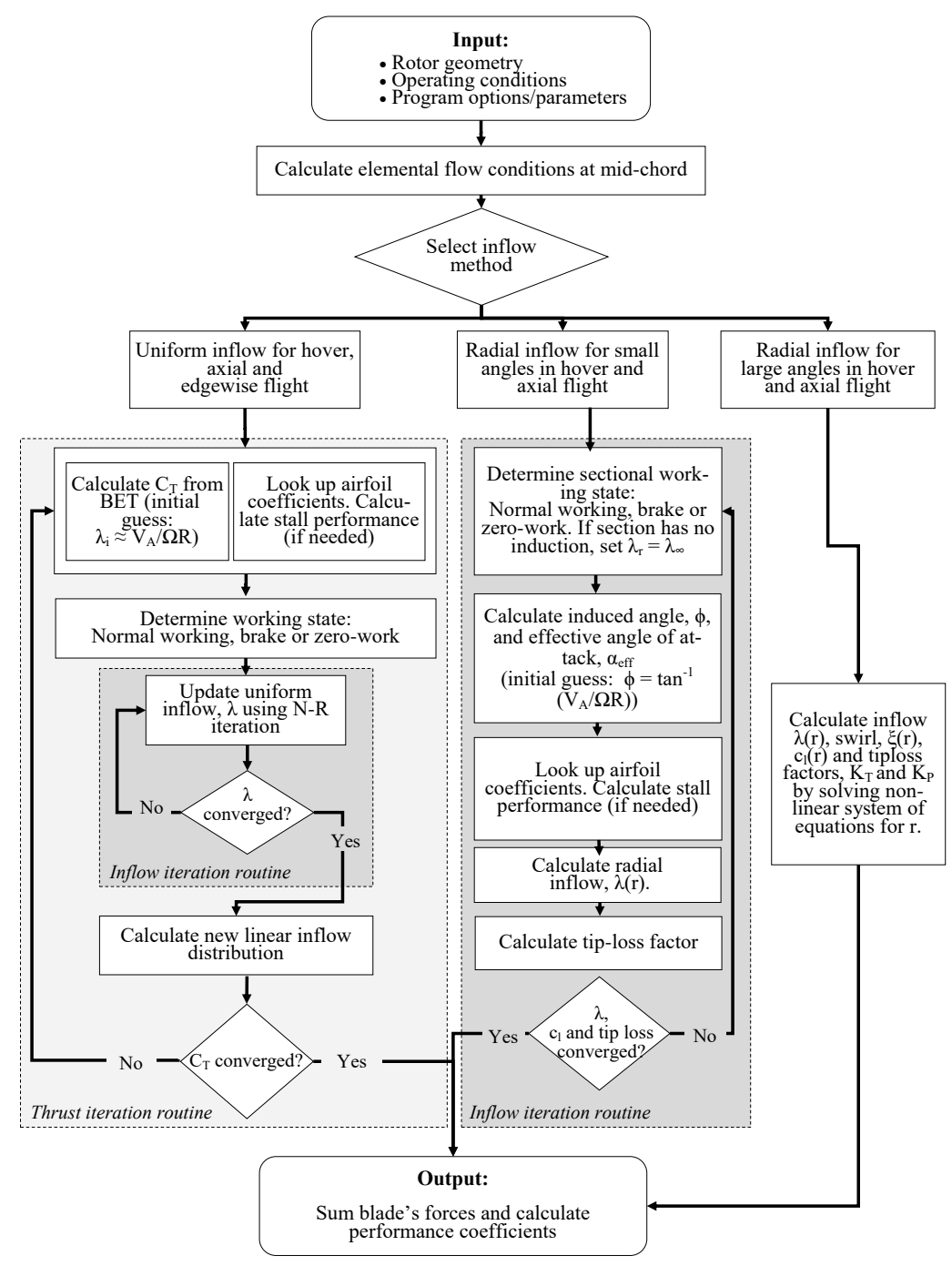

Figure 3.8: Inflow routine block diagram.

To calculate the performance of multiple rotors in configuration, two additional algorithms are developed and shown in Figs.3.9 and 3.10. The purpose of these is so that, on a vehicle level, the collective in-flight performance of multiple rotors and their resulting wake structures can be estimated. Both algorithms consist of the single rotor performance routine combined with the vortex wake routine into a general algorithm.

The first algorithm, shown in Fig.3.9, uses an input of rotor-shaft speed. The process iterates until convergence for each of the rotor's thrust is met or until the maximum number of iterations 
threshold is exceeded. For most flight conditions, convergence is relatively fast due to the similarities between the initial wake geometries compared with the final ones, which reflect the interference effects from the other wakes. This is because the wake induced velocities have a relatively small effect on the thrust produced for a given shaft speed. Using a similar approach, an investigation into the induced wake effects for several vehicle configurations and on a flight performance level is performed in Ref.60.

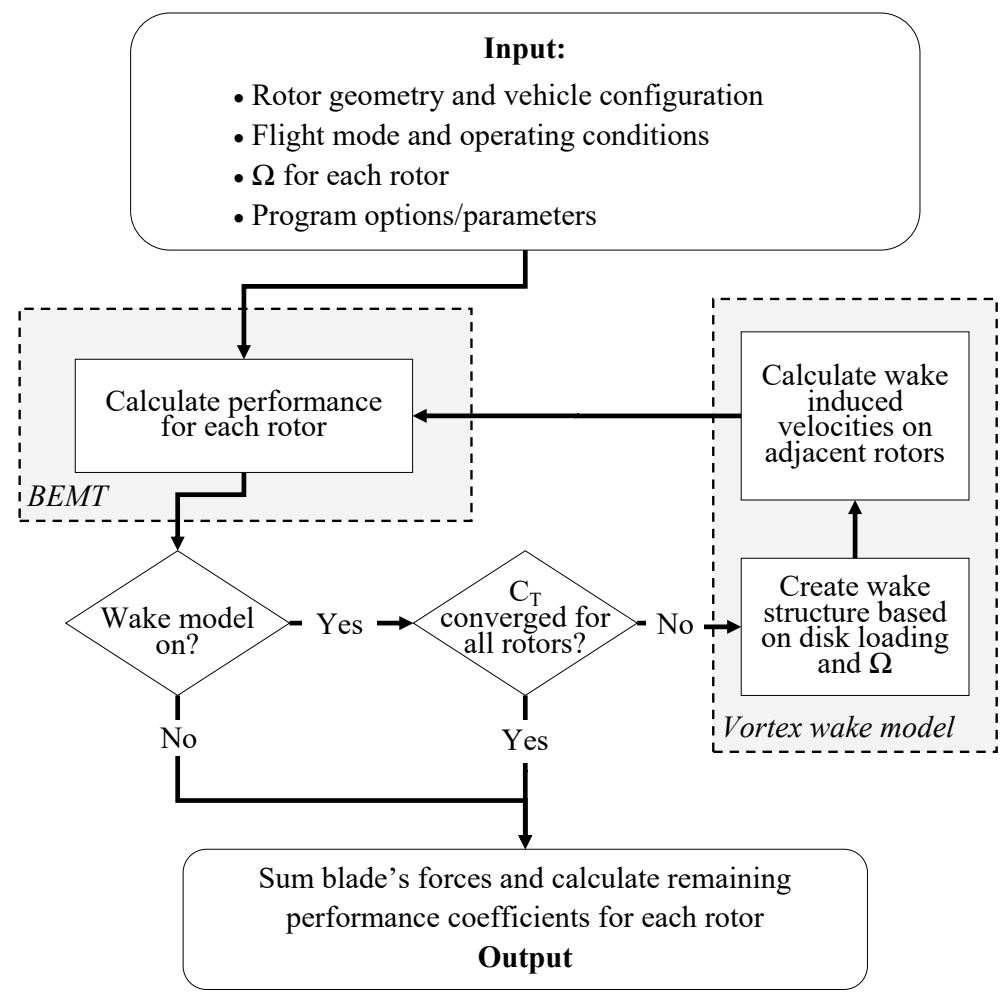

Figure 3.9: Flow chart outlining the routine for the multirotor aerodynamic performance program with input: $\Omega=\left[\Omega_{1}, \Omega_{2} \ldots \Omega_{n}\right]$.

The second algorithm, shown in Fig. 3.10, uses the input of the required thrust for each rotor. The trim subroutine, which determines the shaft speed that produced the required thrust, is implemented using MATLAB's non-linear least-squares solver. For the vehicle to maintain its orientation in steady flight, the cumulative forces and moments created by the rotors and the 
vehicle's airframe must sum to zero. An approach to trimming the entire vehicle is a considerable task on its own and is outside of the scope of this thesis, however, this approach can be used to investigate some of the induced wake effects when the required thrust of each rotor is already known.

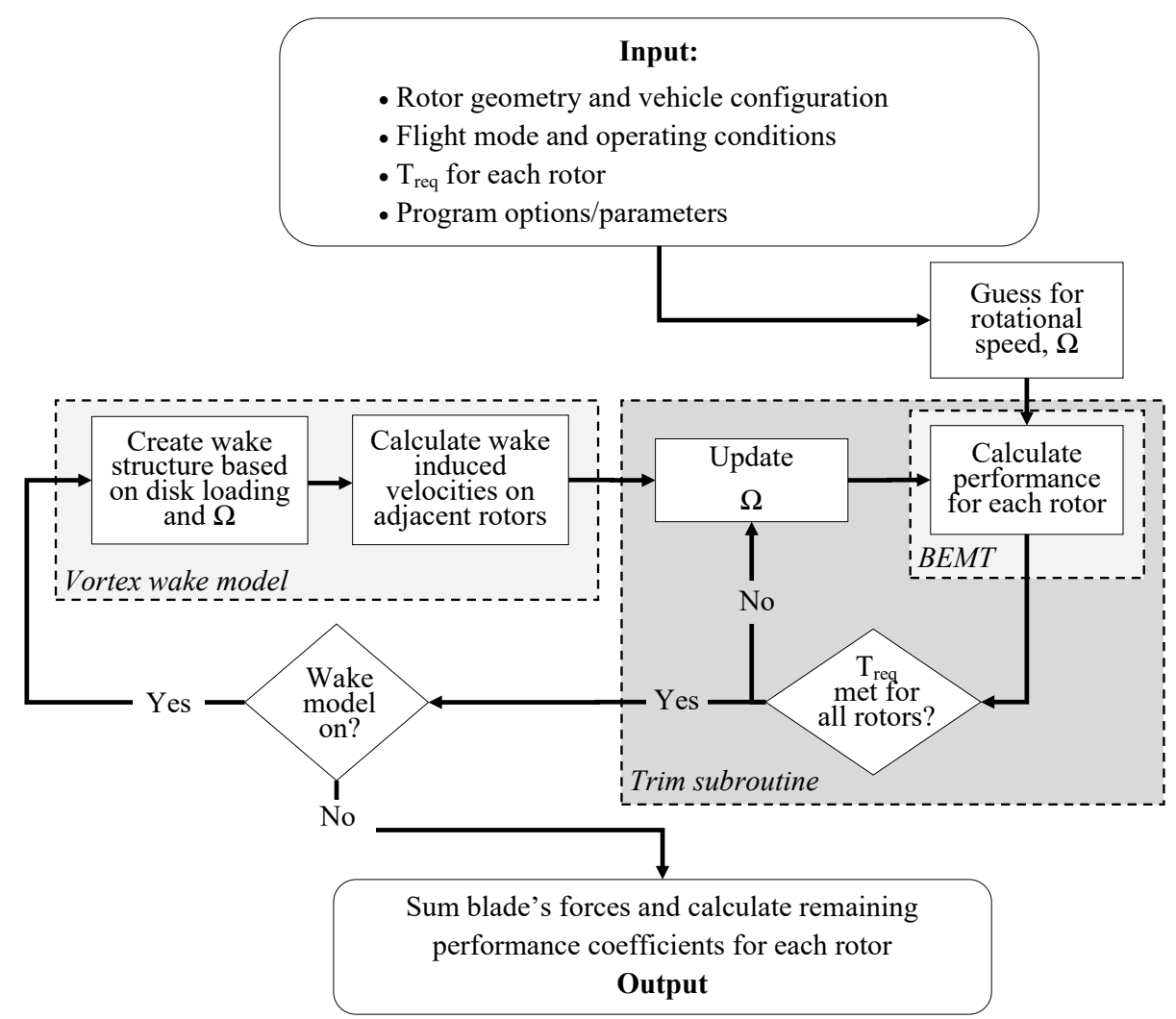

Figure 3.10: Flow chart outlining the routine for the multirotor aerodynamic performance program with input: $T_{\text {req. }}$. 


\subsubsection{Blade Geometry and Section Characteristics}

To determine the sectional characteristics of pre-fabricated blades, a laser scanner is used to create a three-dimensional CAD model. Fig.3.11 shows a model generated for a representative blade with a cut-away section outlined in red. To determine the sectional properties of a blade, the shapes from several of cut-away sections along the blade are extracted, normalized and smoothed. The section's thickness and camber are then compared to a data base of airfoils, many of which are from Ref.17. For shapes that do not have a match, their aerodynamic coefficients are computed using the theoretical prediction methods discussed in Sec.3.3.8.
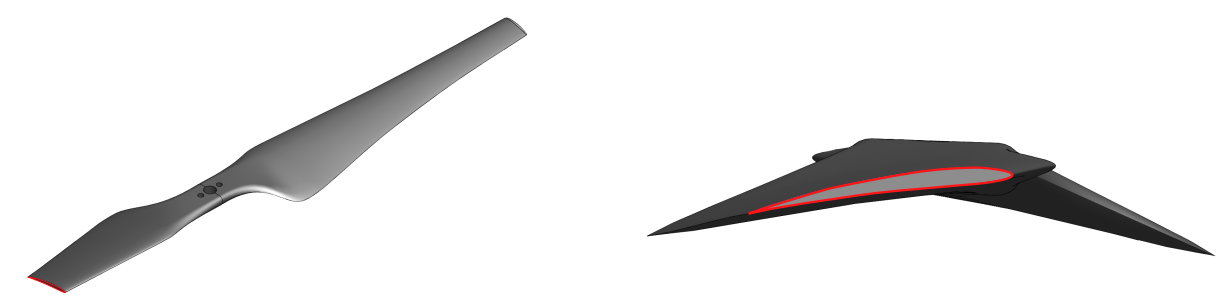

Figure 3.11: Three dimensional computer model of a representative rotor blade with cut-away section outlined in red. 


\section{Chapter 4}

\section{Experimental Determination of Small Rotor Performance}

\subsection{Experimental Set-up}

A three-degrees of freedom rotor/propeller test stand and data collection apparatus were built in order to determine the thrust, torque and pitching/rolling moment during in-flight conditions. The stand is located inside the test section of Ryerson University's subsonic closed-circuit wind tunnel. Fig.4.1 shows the test stand installed in the test section. The base of the test stand is secured to a turn table that rotates 360 degrees.

Referring to the labelled diagram in Fig.4.2, the motor is fastened to a firewall that is fixed on the end of an 8 in shaft. The shaft sits on an aluminium chassis and is held in place by two linear roller bearings. The shaft is attached to a load cell that measures the rotor-thrust force. A torque arm is attached to the shaft at its approximate midpoint. The torque arm is attached to another load cell that records the rotor torque. The entire thrust-torque assembly is mounted to the main support platform using a pivot and another load cell, which records the rolling moments that the rotor develops due to advancing/retreating blade effects. The sensor assembly on the main support platform is encompassed by a fiberglass cowling. The rotor's rotational speed is measured using an infrared sensor. 

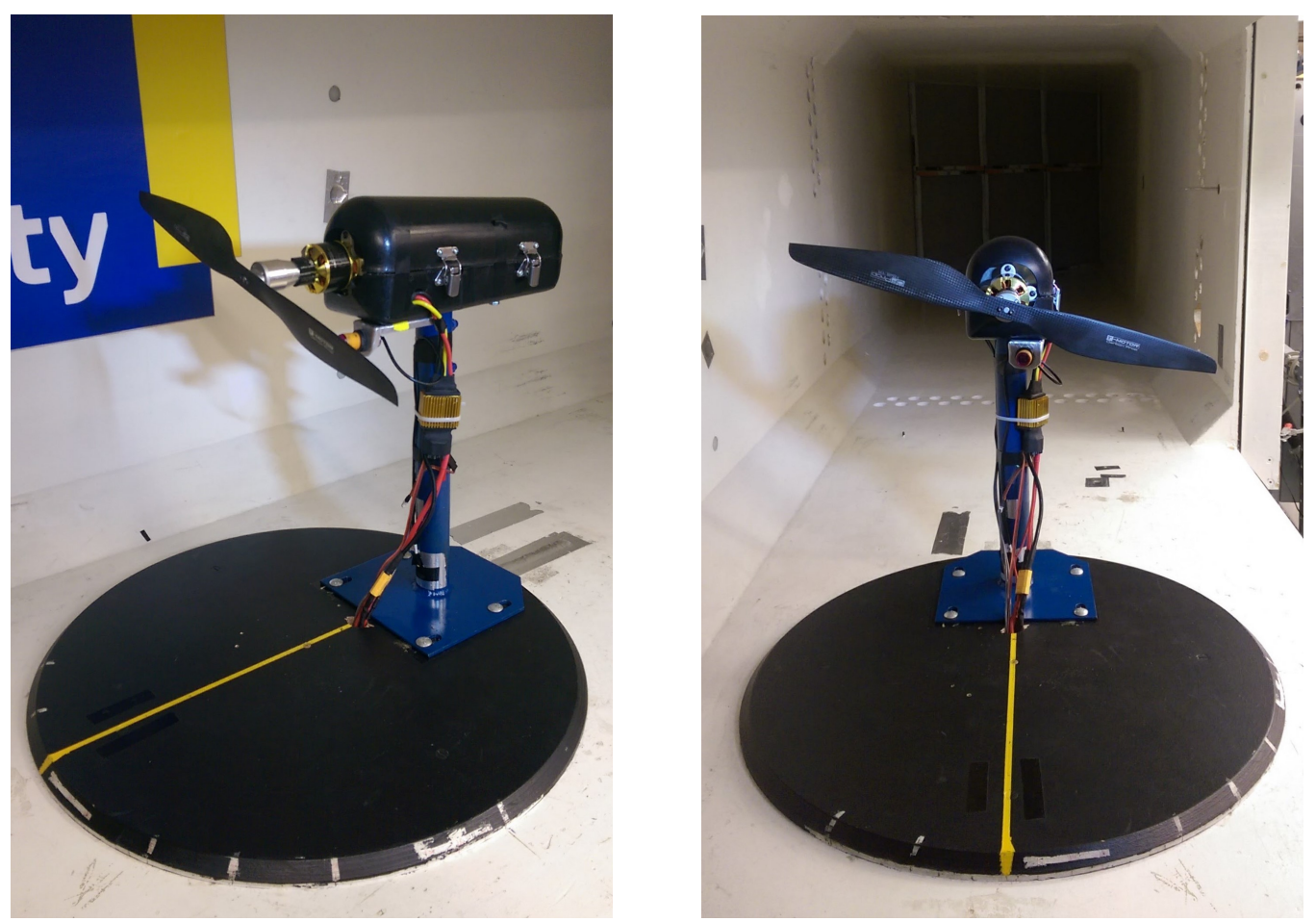

Figure 4.1: Rotor test stand installed in wind tunnel test section with fairing installed.

The electronic speed controller, also shown in Fig.4.2, is attached to the stand support arm with its heat fins directed towards the freestream to allow for adequate cooling. The wire connectors between the test stand components and the data acquisition system run internally within the stand's support shaft, whereas the wires responsible for delivering power through the electronic speed controller and to the motor are run along the outside of the stand. A quick connector is located at the base of the stand and can be easily disconnected when the apparatus requires adjustment. During testing, loose wires along the test stand are secured with tape. 


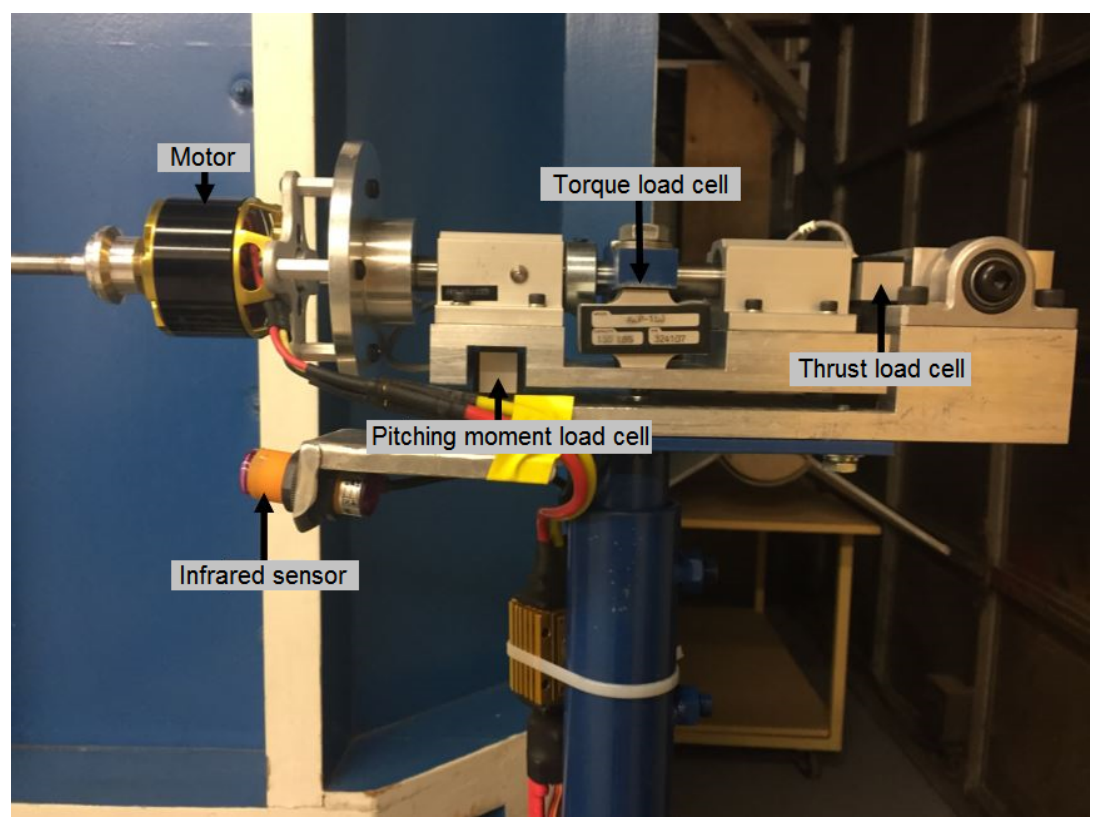

Figure 4.2: Test stand apparatus with components labelled

Power is supplied to the motor using an adjustable 1200 Watt, 50-Amp AC/DC power supply. The power draw is proportional to the throttle signal generated by an Arduino UNO microcontroller running a custom-made throttle control program written in the Arduino Integrated Development Environment (IDE). The micro-controller is connected to a laptop which supplies power and runs the IDE terminal allowing the user to input a throttle setting in percentage. The load cells are connected to load cell conditioners which are in turn connected to the LabJack T7 data acquisition system (DAQ). Each sensor has a calibration formula which is modified before each test to reflect the ambient conditions and the sensor zero-load tare. The DAQ is connected to the laptop which runs the LabJack LJLogM software at a sampling rate of $5000 \mathrm{~Hz}$. The calibrated readouts are stored in a log file, which is post-processed using a MATLAB script that reduces the measured thrust, torque and moment into coefficient form.

The components used for the stand and DAQ are listed in TableB.1.1 TableB.1.2. An uncertainty analysis was performed for the sensors used in addition to the measured performance coefficients. These are summarized in TablesB.1.3 and B.1.4. 


\subsubsection{Wind Tunnel Facility}

A schematic of the wind tunnel is shown in Fig.B.2.1. The test section has a square cross section measuring $0.9 \mathrm{~m} \times 0.9 \mathrm{~m} \times 1.5 \mathrm{~m}$. The wind tunnel is capable of producing airspeeds from $2.8 \mathrm{~m} / \mathrm{s}$ to $50 \mathrm{~m} / \mathrm{s}$ in the test section and is controlled by adjusting the rotational speed of a $1.23 \mathrm{~m}$ diameter fan powered using a 1500 horsepower AC motor. The flow velocity in the test section is determined by measuring the static pressure drop in the contraction section. A hand-held pitot-static tube was also used to verify the measurements made by the static ports. To improve flow quality, the tunnel is equipped with 3 anti-turbulence screens before the test section in addition to turning vanes positioned at the corners. The wind tunnel has a turbulence intensity level of $0.2858 \%$ that was determined using a hot-wire anemometer [62].

\subsubsection{Testing Procedure}

Prior to attaching the rotor, load cell measurements are taken at several airspeeds speeds in order to determine their base drag loads. These base-load measurements are used to correct the sensor loads that are measured when testing. The base-drag measurements are also corrected for the rotor's induced velocities using a momentum theory-based approach, where:

$$
v_{i}=\frac{1}{2}\left[-V_{\infty}+\sqrt{V_{\infty}^{2}+\frac{2 T}{\rho A}}\right]
$$

Eq.4.1 is used to approximate the drag force caused by the rotor wake impinging on the firewall and is subtracted from the measured thrust.

Ensuring that the rotor is properly secured, the throttle for the test stand motor is increased to a near-peak setting, held for approximately 10 seconds, and then slowly brought down to zero. The load cell calibration formula's are adjusted to reflect the tare weight. Performance data is collected over a range of advance ratios by holding the rotor's rotational speed constant and increasing the airspeed in the test section. Minor adjustments to the test stand motor's throttle must be made throughout the experiment to ensure that the rotor's rotational speed is maintained. This method is preferred over the method where the test section velocity is held constant for 
two reasons that were discovered during testing. The first is that making major adjustments to the rotor's rotational speed has a significant effect on the load cell calibration. Secondly, the rotational speeds which coincide with the harmonic resonance modes of the test stand, and thus have considerably more vibrations, can be avoided.

\subsection{Test Stand Validation}

To validate the test stand, the thrust and power measurements made using a Master Airscrew $11 \times 7$ electric were compared to the measurements made at another facility $[40,63]$. The MA11x7E is a hobby propeller designed for electric brushless motors and was selected due to it being representative of the sectional Reynolds numbers and rotational speeds expected for small multicopter rotors.

Figures 4.3 through 4.6 show the comparison between thrust and power measurements made at static conditions using the Ryerson facility and the one that is discussed in Ref.40. The two data sets, indicated by the respective lines, were collected at two different times, each requiring the stand to have been re-positioned and re-calibrated. The purpose of this was to check the repeatability of the measurements. 


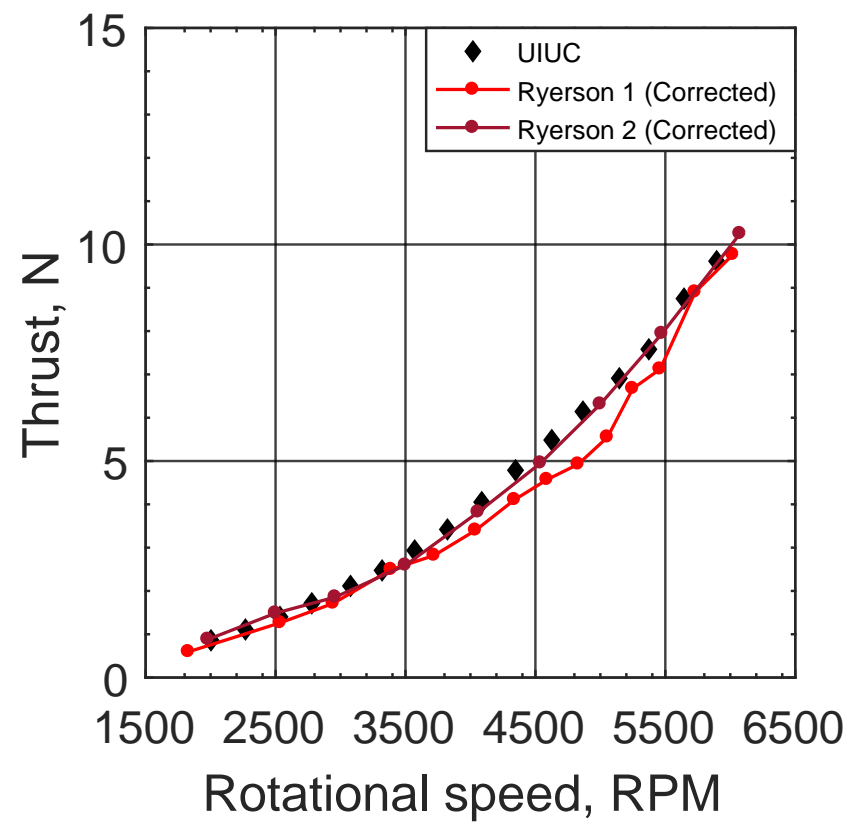

Figure 4.3: Thrust vs. rotational speed at static conditions measured using the Ryerson test stand and compared with Ref.40.

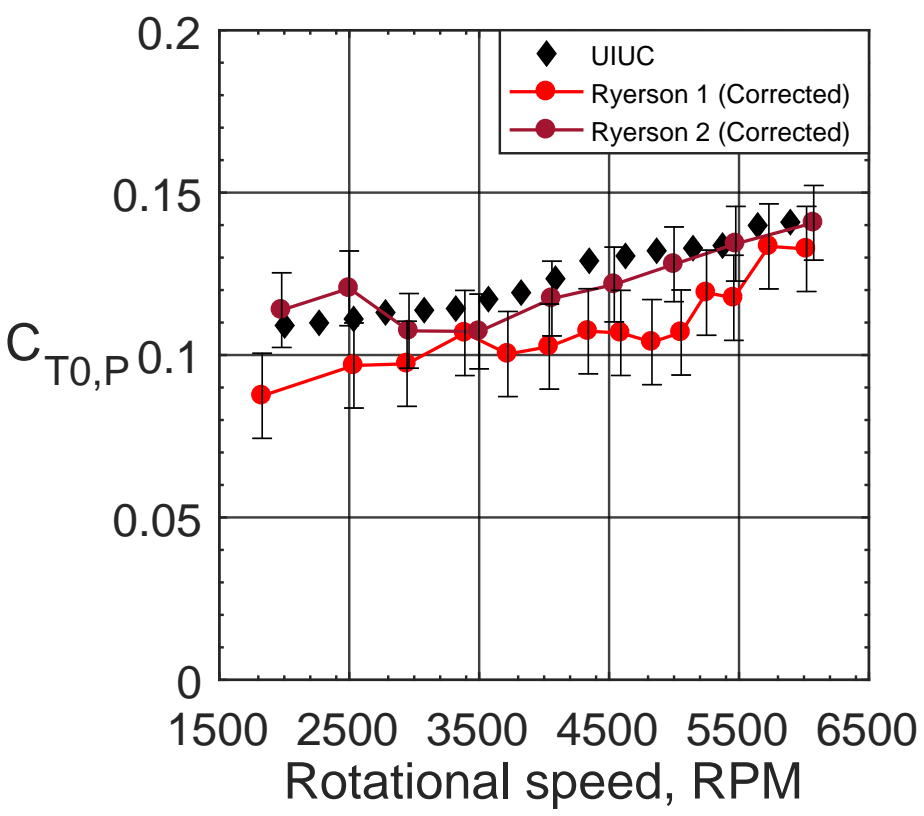

Figure 4.4: Thrust coefficient vs. rotational speed at static conditions measured using the Ryerson test stand and compared with Ref.40. 


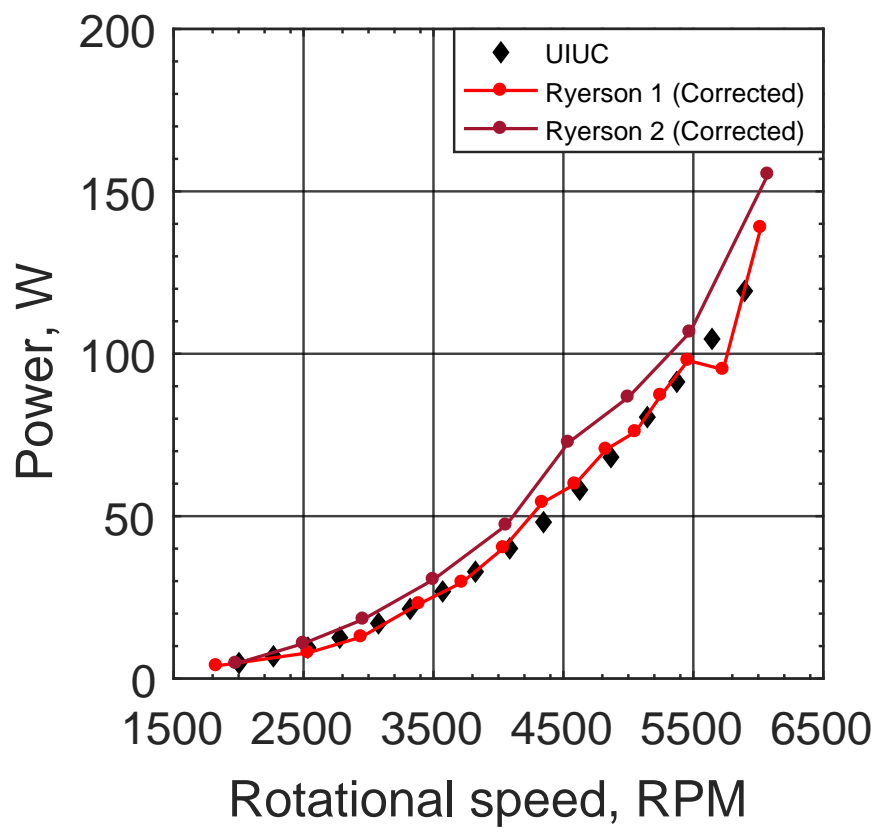

Figure 4.5: Power vs. rotational speed at static conditions measured using the Ryerson test stand and compared with Ref.40.

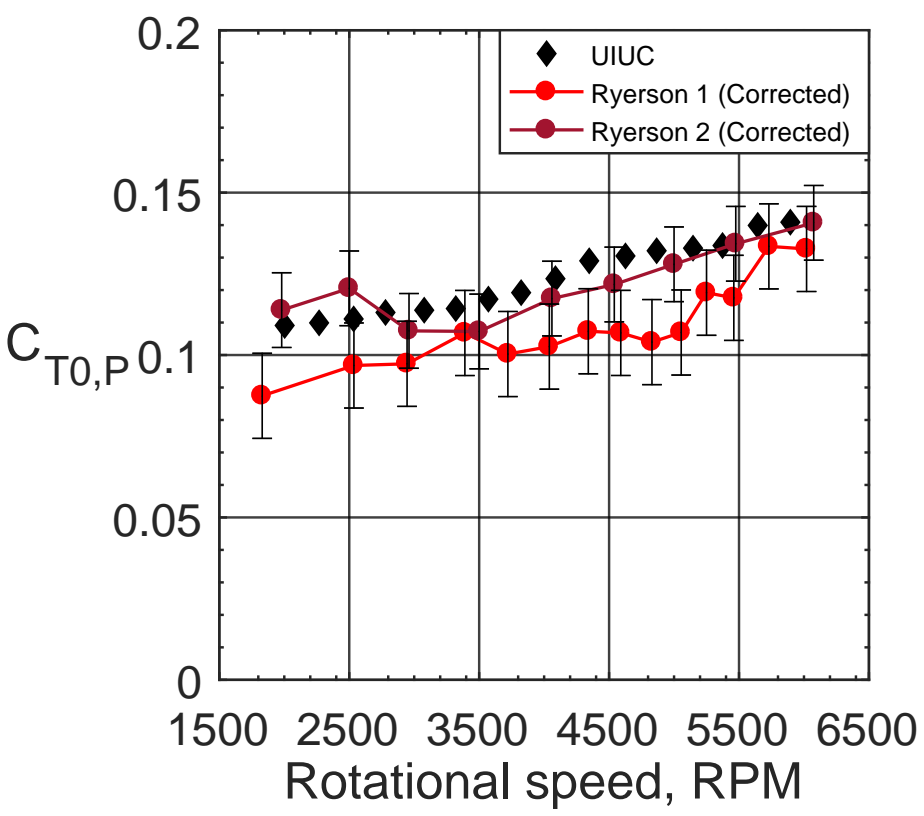

Figure 4.6: Power coefficient vs. rotational speed at static conditions measured using the Ryerson test stand and compared with Ref.40. 
The observed thrust and power follow comparable trends although the Ryerson test data have more scatter. An explanation for this is discussed in Sec.4.3. Despite some of the differences, much the measured data taken using the Ryerson facility is within one standard deviation of the the data from Ref.40, as shown by the error bars in Figs 4.4 and 4.6. In particular, the offset between the measured thrust and power is consistent between the two data sets, thus, the measurements from the Ryerson apparatus can be used to experimentally validate the rotor prediction methodology in Sec.3.3 for static conditions.

Figures 4.7 and 4.8 show the thrust and power coefficients for a range of advance ratios. The solid coloured lines represent the measurements made by the Ryerson test stand and correspond with the ones made using the apparatus in Ref.40 at the same rotational speeds. The results presented here were collected at the same time as run 2 for the static tests.

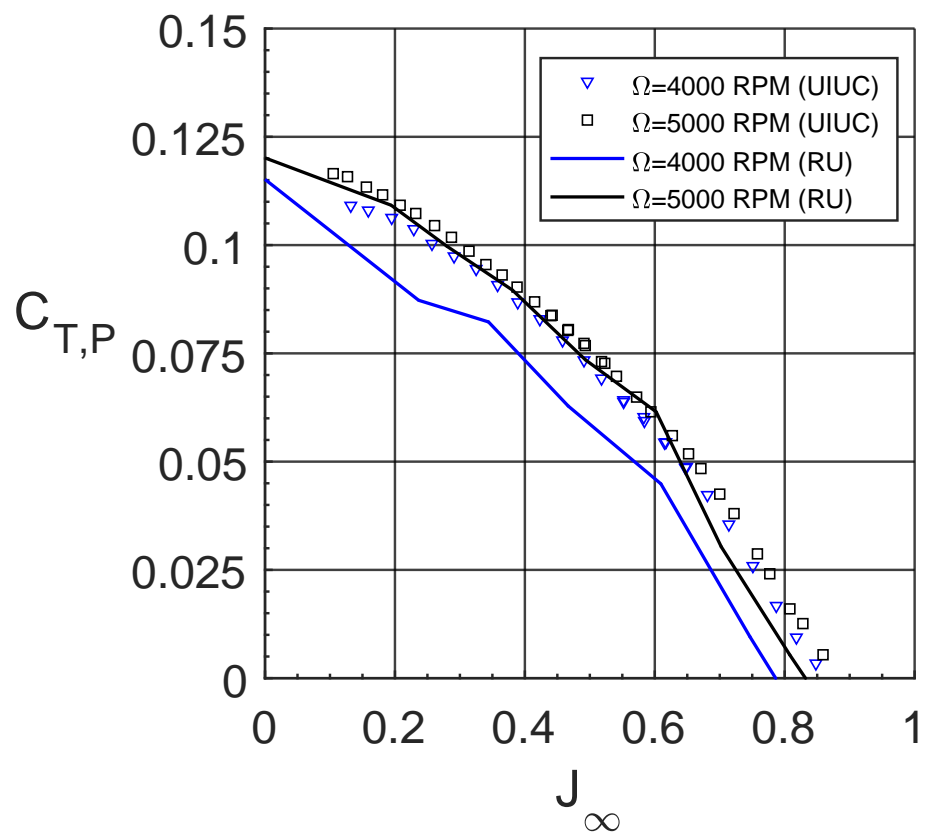

Figure 4.7: Thrust coefficient vs. advance ratio measured using the Ryerson test stand compared to measurements made in Ref.40.

There are several differences between the measurements made by the Ryerson test stand and the measurements in Ref.40. Most noticeably is the larger variance between different rotational 
speeds, and an over prediction in the power. The cause of these differences is also discussed in Sec.4.3. Despite this, the measurements from both facilities show acceptable agreement, in particular, the thrust and power when the propeller stalls is located at approximately the same advance ratio. Furthermore, both data sets measure a similar trend throughout the tested advance ratios. For these reasons, the test stand can be used with some degree of confidence to validate the rotor prediction methodology at forward ratios of advance.

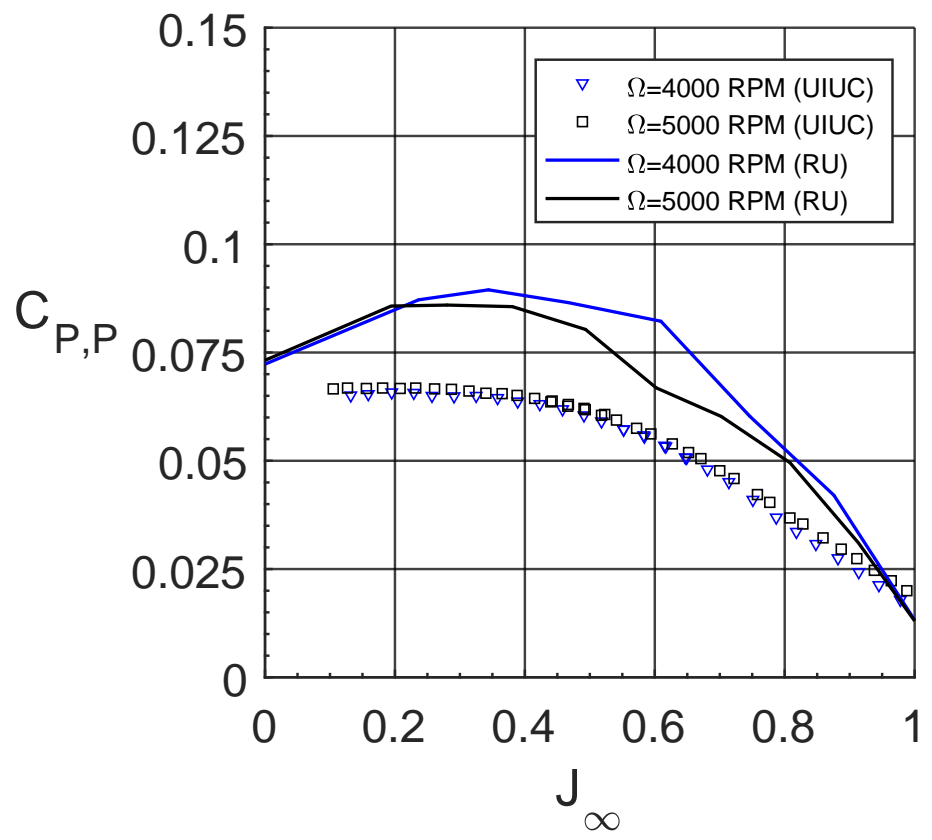

Figure 4.8: Power coefficient vs. advance ratio measured using the Ryerson test stand compared to measurements made in Ref.40.

\subsection{Systematic Sources of Error}

The scatter observed with the Ryerson apparatus is presumably caused by a number of systematic sources of error: The first is that the measured forces are small compared to the full-scale load of the load cells and, in some cases, are within an order of magnitude of their error specification. Referring to the uncertainty analysis in Table B.1.3, the percentage error for all three of the load cells is $0.6883 \%$. For the $50 \mathrm{lb}$-max thrust load cell, this translates to a full-scale load error of $\pm 1.53 \mathrm{~N}$ and $\pm 4.593 \mathrm{~N}( \pm 0.1167 \mathrm{Nm})$ for the $150 \mathrm{lb}$-max torque load cell. 
For this propeller, the smallest measured thrust is 2 to 3 times greater than the error of the load cell, however, the smallest measurement for torque is within the error specification. Moreover, for the highest torque measurements, they are only 1 to 2 times greater than the error. Sensors of this type, specifically the ones that are designed to sense over large ranges, are susceptible to non-linearities for small measurements, and thus, is likely a large contributing factor to the over-measurement of the power coefficient.

The second factor is related to the method by which torque is measured. The Ryerson set-up measures the force applied through a moment arm to a linear type load cell, whereas the apparatus in Ref.40 stand uses a torque transducer fastened directly to the motor housing. The Ryerson apparatus is more susceptible to compounding residual errors associated with having multiple moving components, each with their own tolerances, that are used in conjunction. As a consequence, test stand vibrations have a larger effect on the Ryerson set-up and is investigated in the following section. Another source of error is that each apparatus used separate motors, and therefore, small differences are possible in their efficiency characteristics, including differences in their internal frictions and moments of inertia.

Ultimately, resolving these systematic sources of error requires that some of the test stand components be replaced. For instance, torque should be measured with a torque transducer that has a smaller range and is located directly between the motor and the firewall. The shaft used to transmit the thrusting force can then be significantly shortened and constrained so that it only allowed to move in the axial direction. However, due to the limited time-frame and budget for this project, these improvements are not implemented in the present work.

\subsubsection{Signal Analysis and Vibrations}

To further investigate the performance of the test stand, a spectral analysis was performed on the raw output signals for each of the load cells. The purpose of this is to investigate the test stand vibrations. Figures 4.9 through 4.11 show representative raw signals for thrust, torque and pitch load cells, respectively. The data was collected for 6 seconds at a sampling rate of $5000 \mathrm{~Hz}$ and the propeller was operating in static conditions at a rotational speed of $3074 \mathrm{rpm}(51 \mathrm{~Hz})$.

Although the signals collected are relatively noisy, performing a fast Fourier transformation, 
shown in Figs.4.12 through 4.14, indicates that the dominant frequencies, for the thrust and pitching moment load cells, correspond to the rotational speed of the motor, as expected. However, the dominant frequency for the torque load cell is located at $60 \mathrm{~Hz}$. This indicates that the components associated with the torque load cell may have electromagnetic shielding issues. Furthermore, it is also subjected to considerably more vibrations, which is shown by the large number of erratically spaced vibration amplitudes, compared to the other two load cells. Thus, the measurements taken for torque and power are less reliable than for the thrust and pitching moment.

The frequencies observed for the pitching moment load cell, shown in Fig.4.14, provide a representation of the resonance frequencies of the test stand. This is because at static conditions, the propeller's pitching moment is negligible. In this case, the dominant are equally spaced at intervals of $51 \mathrm{~Hz}$ which corresponding to the motor's rotational speed.

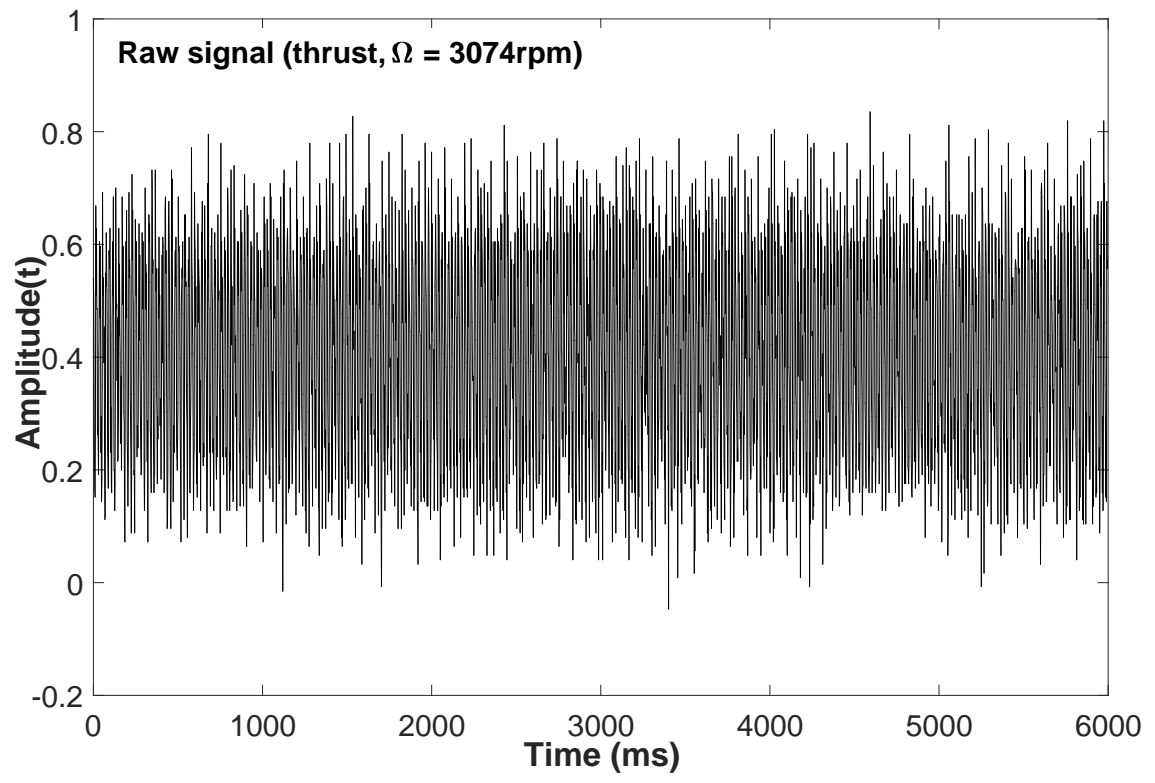

Figure 4.9: Representative raw signal for thrust load cell (MA11x7E, $n=3074 \mathrm{rpm}$ ). 


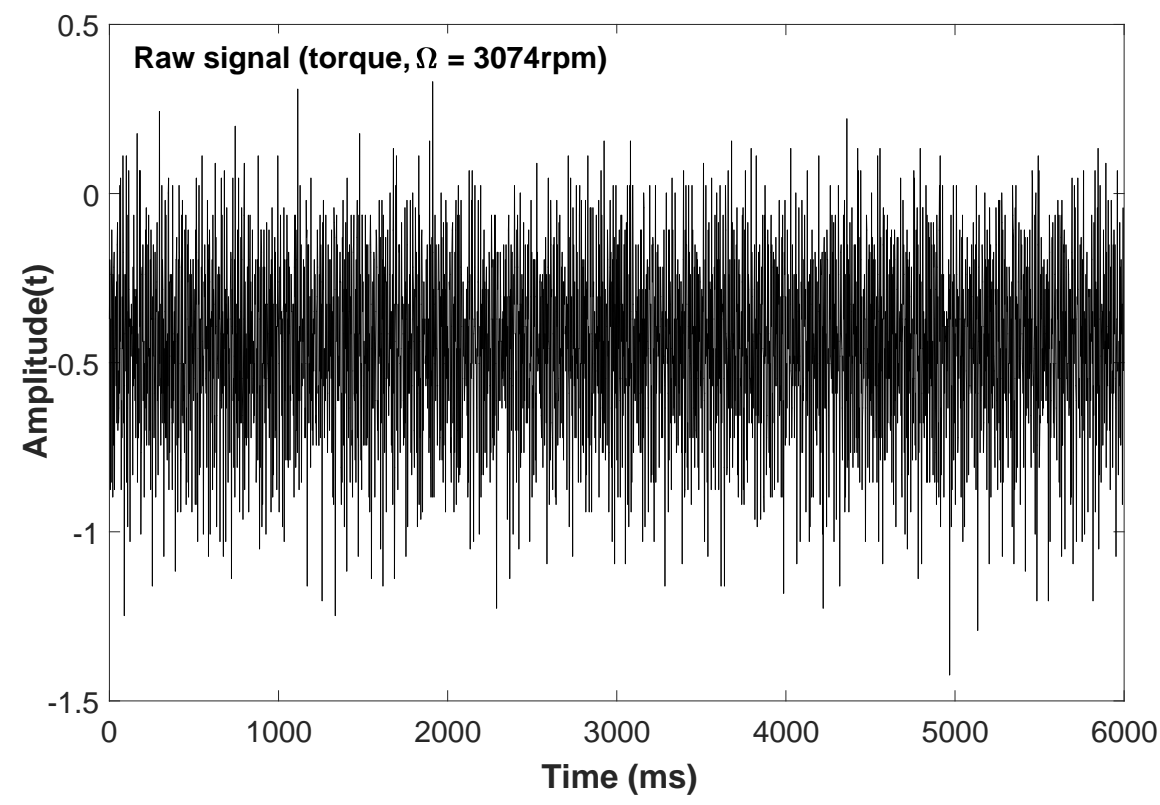

Figure 4.10: Representative raw signal for the torque load cell (MA11x7E, n=3074rpm).

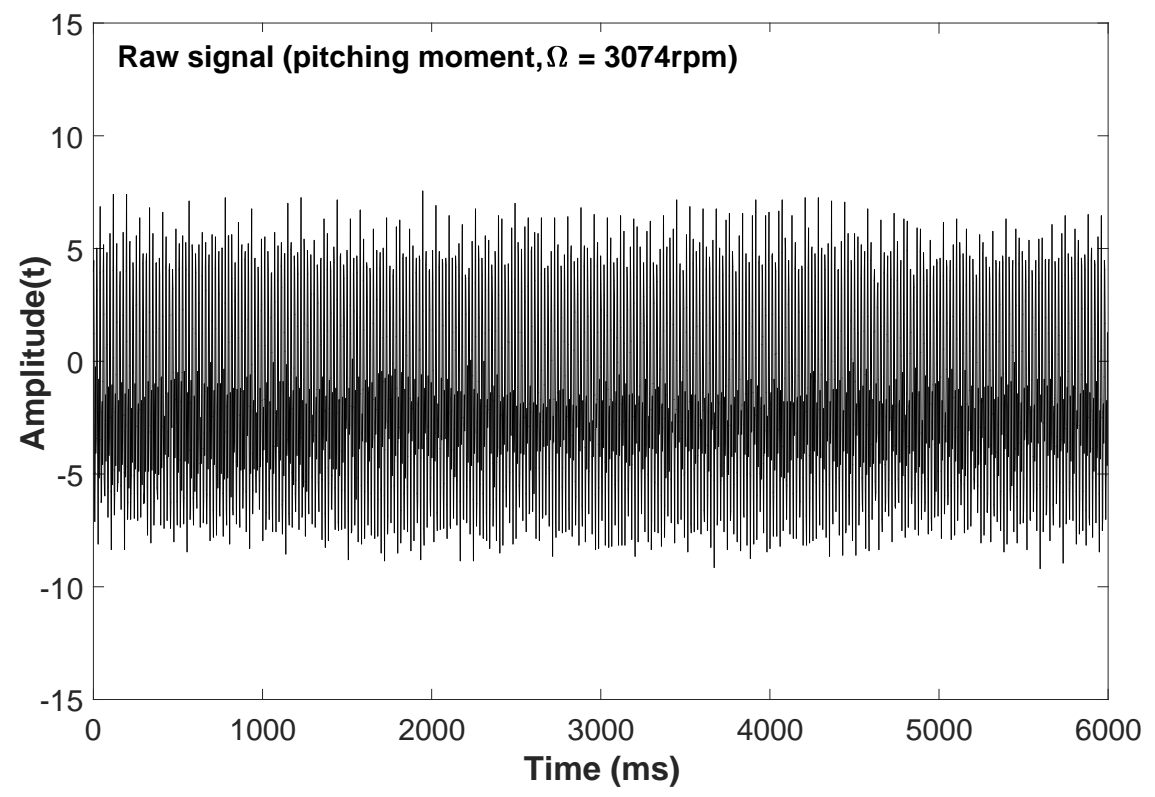

Figure 4.11: Representative raw signal for the pitching moment load cell (MA11x7E, $\mathrm{n}=3074 \mathrm{rpm})$. 


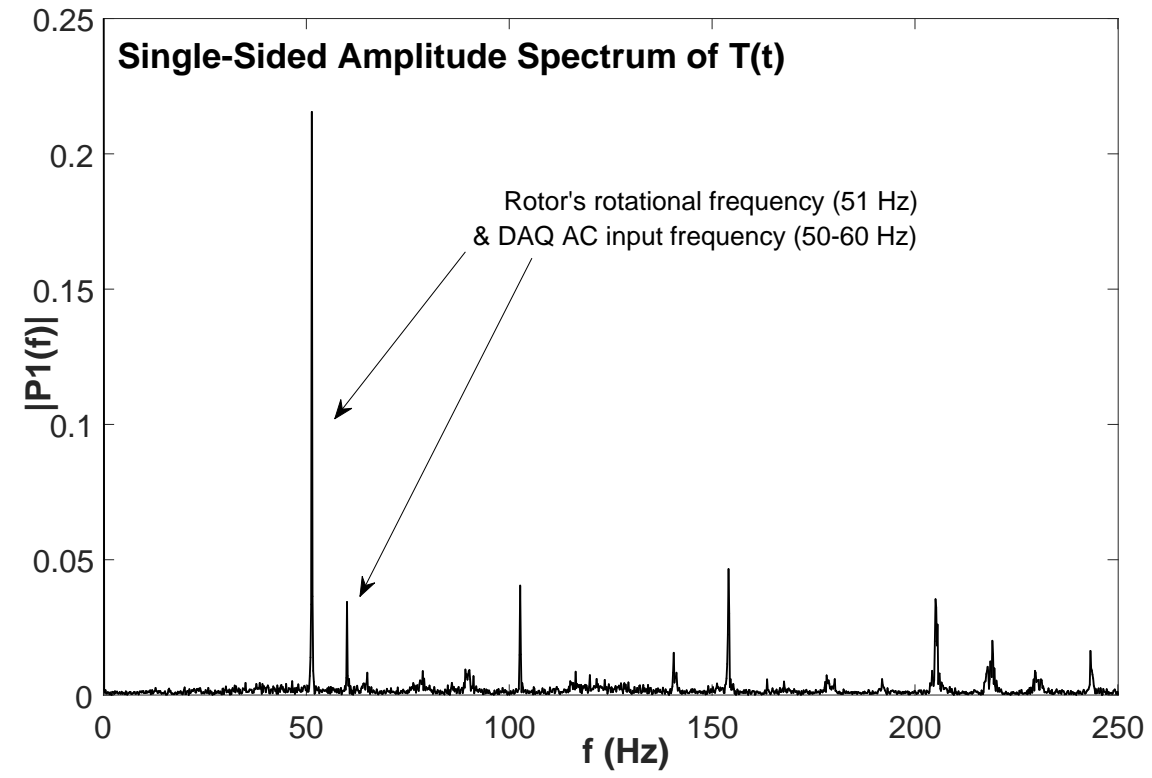

Figure 4.12: Harmonic vibration amplitudes observed by the thrust load cell.

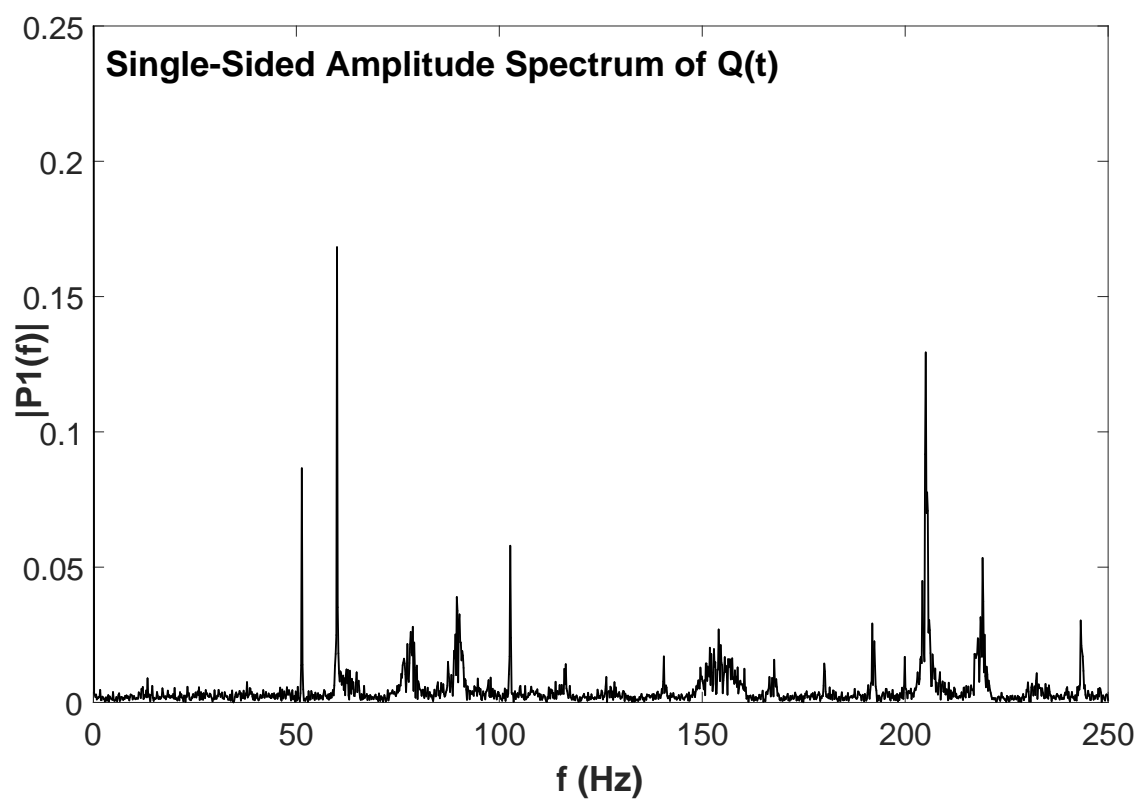

Figure 4.13: Harmonic vibration amplitudes observed by the torque load cell. 


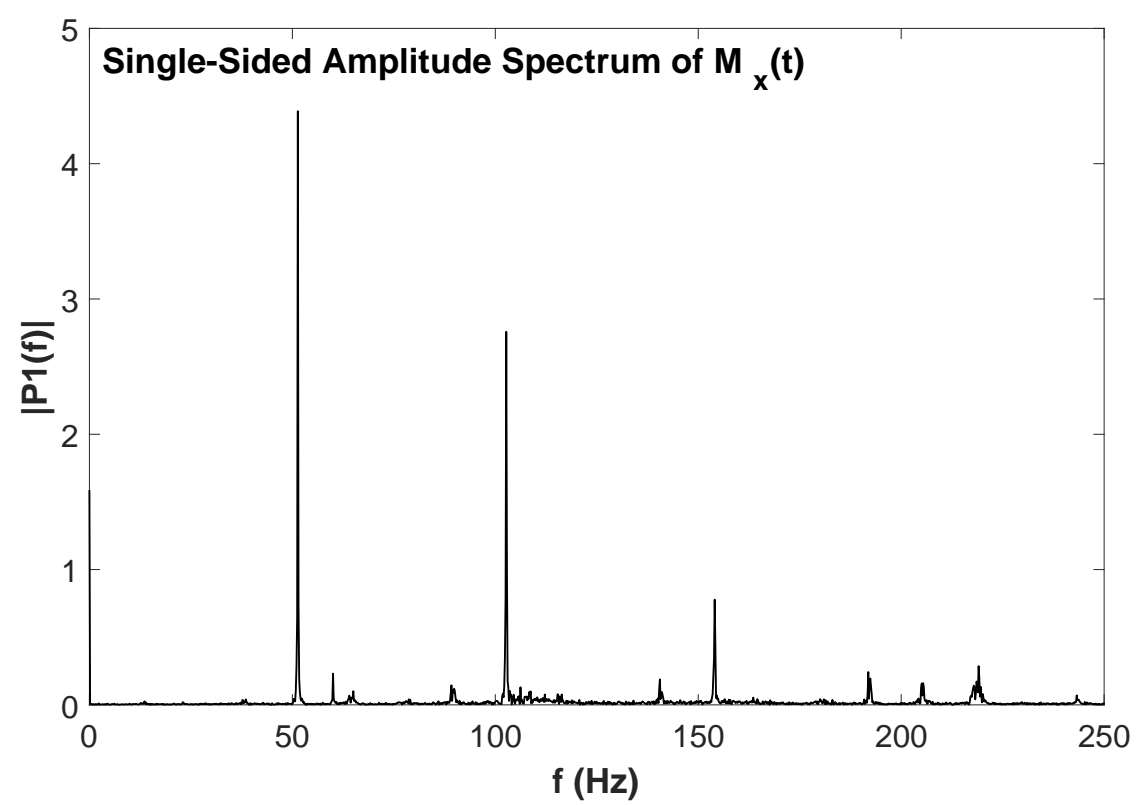

Figure 4.14: Harmonic vibration amplitudes observed by the pitching moment load cell.

\subsubsection{Wind Tunnel Wall Interference}

In edgewise flight, some of the rotor wake can impinge on the wind tunnel walls, as shown in Fig.4.15. For low freestream advance ratios, this results in an increase in measured thrust. This effect is analogous to the rotor operating in ground effect. It is suggested in Ref.13 that the minimum ground clearance for there to be no interference effects, for full-scale rotors, is approximately eqaul to the rotor diameter. Using this guideline and based on the position of the test stand in the test section, the maximum allowable diameter for there to be negligible interference effects is $0.45 \mathrm{~m}$ (17.7 in). Of the rotors and propellers tested in this thesis, the largest diameter used was 18 in. For small rotors, however, their wakes often contract at a much fast rate than full-scale rotors [13] and so in order to assess the influence of the wind tunnel walls, static tests were performed at several rotor orientations. 


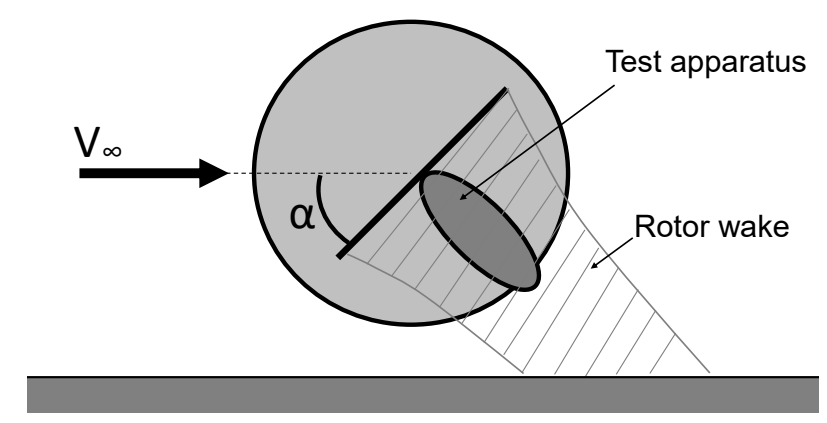

Figure 4.15: Representation of the rotor test stand oriented for edgewise flight testing in wind tunnel test section.

In practice, interference effects can be thought of as a reduction in required power for a given thrust. However, the power supplied to the brushless motor is a function of throttle setting, thus, wall interference results in an increase in thrust for a given throttle setting. The thrust coefficients measured for the T-motor $18 \times 6.1$ in static conditions at several angles of attack and rotational speeds are plotted in Fig.4.16.

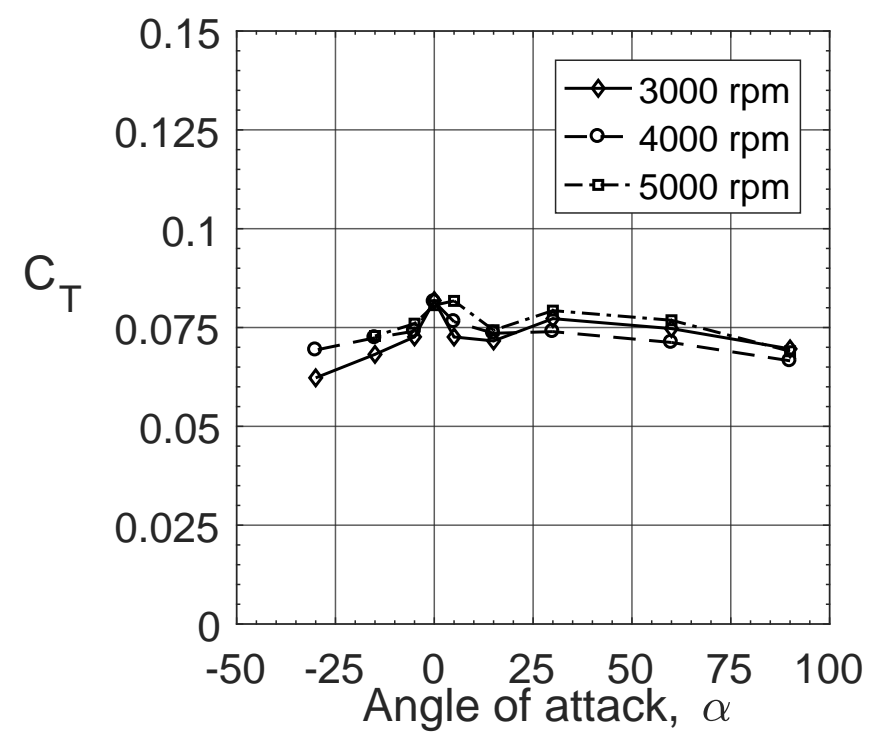

Figure 4.16: Thrust coefficient vs. test stand angle of attack for the T-motor $18 \times 6.1$ operating at static conditions. 
The highest thrust coefficient corresponds with $\alpha=0^{\circ}$ for all of the rotational speeds. The static thrust between the $90^{\circ}$ and $0^{\circ}$ orientations show there to be a difference of approximately 13.7\%. Thus, for this particular set-up, the minimum distance for which a small rotor observes no interference effects is slightly larger the guideline for full-scale rotors. Despite this, the wall interference effects become significantly reduced as the test section velocity is increased and so corrections for wall interference are neglected. 


\section{Chapter 5}

\section{Validation of the Rotor Performance Prediction Methods}

This section describes the validation of the single rotor performance model, and the verification of the vortex wake model using analytical results.

For the single rotor performance model, the predictions are compared with experimental measurements collected using the rotor test stand. While a great amount of experimental data is available for hobby propellers in axial flight and static conditions [40,63], there is limited performance data for small rotors in edgewise flight. As discussed in Sec.2, there is a need for experimental measurements that encompass the flight conditions associated with cruise. Therefore, as part of the validation process for this thesis, a considerable amount of experimental data was collected. Also included in this section is a comparison between the several approaches that are used to determine the rotor inflow in hover and axial flight, and a sensitivity study for the number of azimuth positions. Following this, an examination of the predicted sectional performance of the rotor blade is presented, in order to verify that the model predicts some of the retreating blade effects in forward flight.

For the vortex wake model, verification is performed using the analytical solution for the velocities induced by a vortex tube [53]. Finding the velocity distributions at the rotor disk plane through experimentation is a formidable task, that is well outside the scope of this thesis, and 
therefore the analytical solution is used for comparison.

\subsection{Rotor Performance Model Validation}

\subsubsection{Hover}

The single rotor performance methodology presented in Chapter 3 uses a number of approaches for prediction the induced velocities in hover and axial flight. Some of these use small angle approximations and neglect the influence of swirl velocities. To examine whether or not these simplifications have a significant effect on the performance predictions, comparisons with experimental measurements from Ref.40, for the static case, are presented in Fig.5.1.

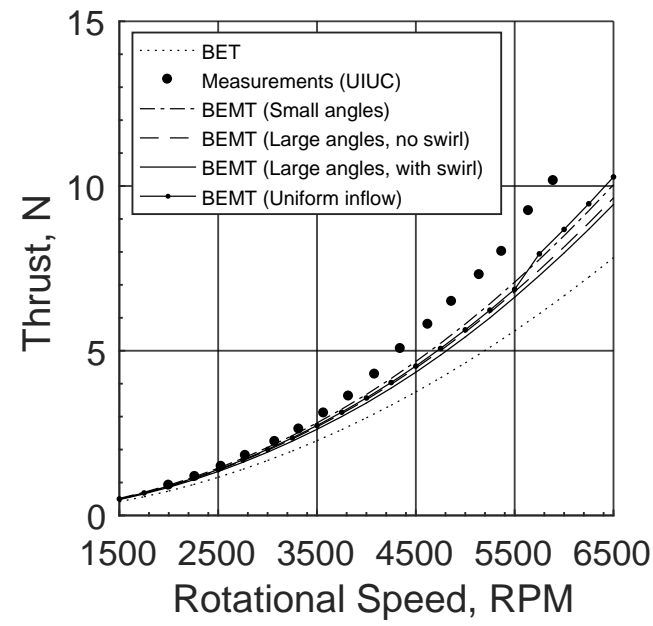

(a) Thrust in static conditions.

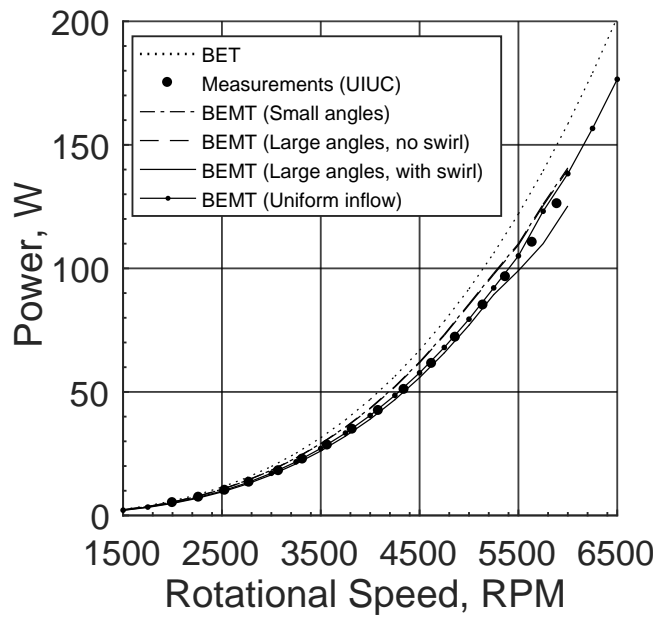

(b) Power in static conditions.

Figure 5.1: Effect of inflow models on the thrust and power predictions for the MA11x7E 2-bladed propeller.

The predicted thrust and power produce similar results for the methods listed in Fig.5.1, and thus, eliminating the small angle approximations and including swirl velocities has a relatively small effect on the predicted hover performance. Furthermore, the approximation of uniform inflow across the rotor disk also predicts similar performance. All of the inflow methods consistently under-predict the thrust for the higher rotational speeds, for which the model predicts that the inboard blade sections are stalled. For instance, at the highest measured rotational speed 
of $6000 \mathrm{rpm}$, the model predicts that $65 \%$ of the blade sections are stalled. Furthermore, the Reynolds numbers over the entire blade are less than $10^{5}$ and so particular features of the blade sections, such as its sharp leading edges, have an effect on the blades stalled behaviour. These effects are not extensively predicted with the sectional aerodynamic coefficient model. Despite this, the power predictions have good agreement throughout the entire range of rotational speeds and all of the inflow methods produce similar trends.

\subsubsection{Edgewise Forward Flight}

In order to validate the model in forward edgewise flight, the experimental performance characteristics of the T-motor 18x6.1 two-bladed rotor were collected for three rotational speeds and up to a maximum freestream advance ratio of approximately $\mu_{\infty}=0.36$. Measurements were taken for several rotor plane angles of attack. The sectional twist and chord distributions of the physical rotor are shown in Fig.A.2.2. The shape of the blade sections were extracted using the method described in Sec.3.5.2 and the sectional aerodynamic coefficients were determined using XFOIL [56].

For the model predictions, the longitudinal and lateral inflow weighing factors are taken from the Pitt and Peters model [54]. The blade forces are determined without using small angles approximations, despite the induced swirl velocities not being explicitly modelled, on the basis that the approximation that the sectional thrust points normal to the rotor plane does not always hold for sections on the inboard blade. The comparison between predictions and measurements are shown in Figs.5.2 through 5.4 for the thrust coefficient and Figs.5.5 through 5.7 for the power coefficient. The predicted thrust coefficients show very good agreement with the measurements, especially for inflow angles greater than $0^{\circ}$. Correlation between measurements and predictions increase with rotational speed and are within 10\% for the positive inflow angles at 5000 RPM. Non-linearities are observed for inflow angles less than $-15^{\circ}$ as the rotor is suspected to approach vortex ring state, in which case momentum theory is invalid until the windmill break state. Despite this, blade element theory provides an estimate of the rotor thrust and produces predictions that are within $15 \%$ of the measurements. 


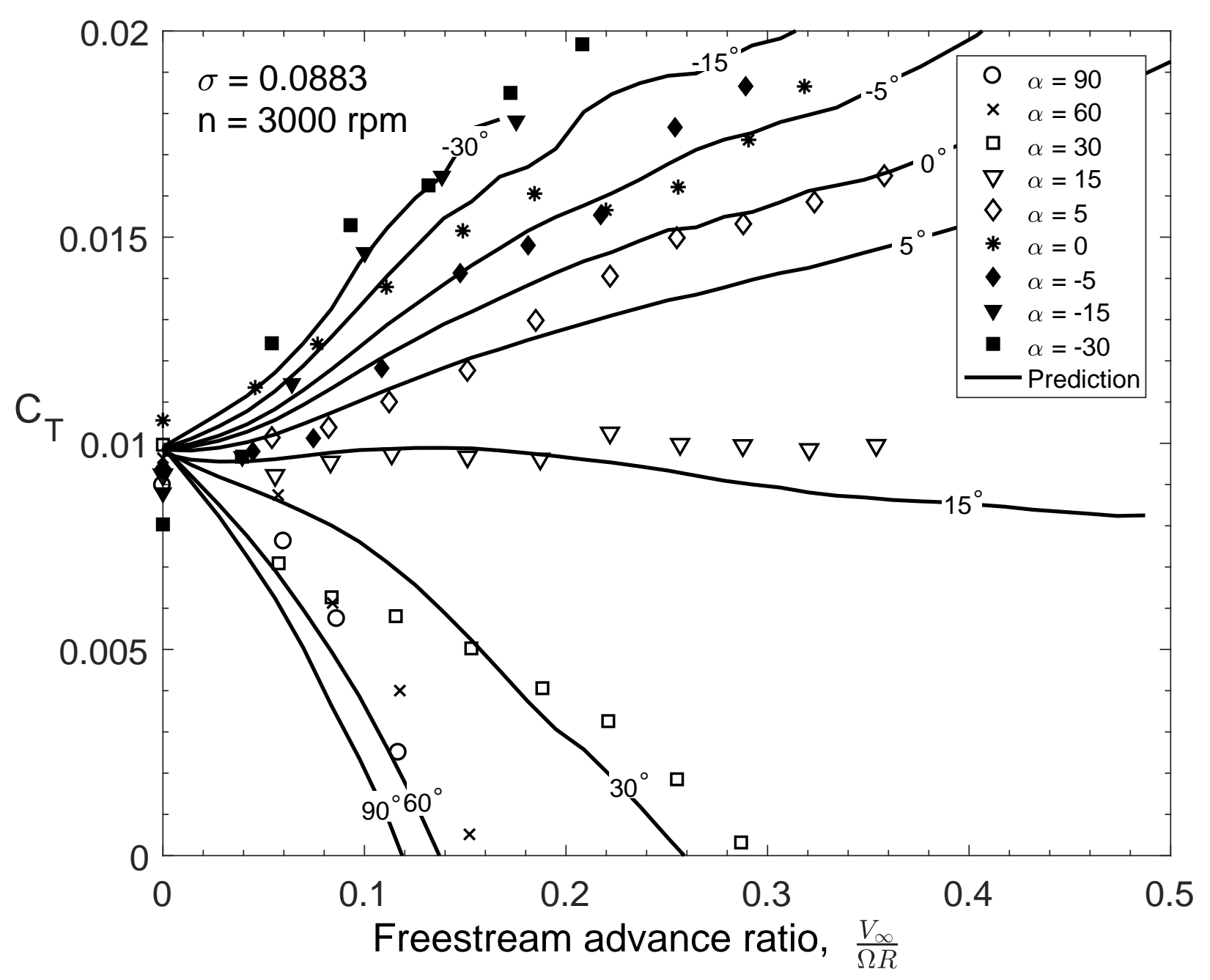

Figure 5.2: Thrust coefficient predictions versus advance ratio for the T-motor $18 \times 6.1$ for several angles of attack, RPM $=3000$. 


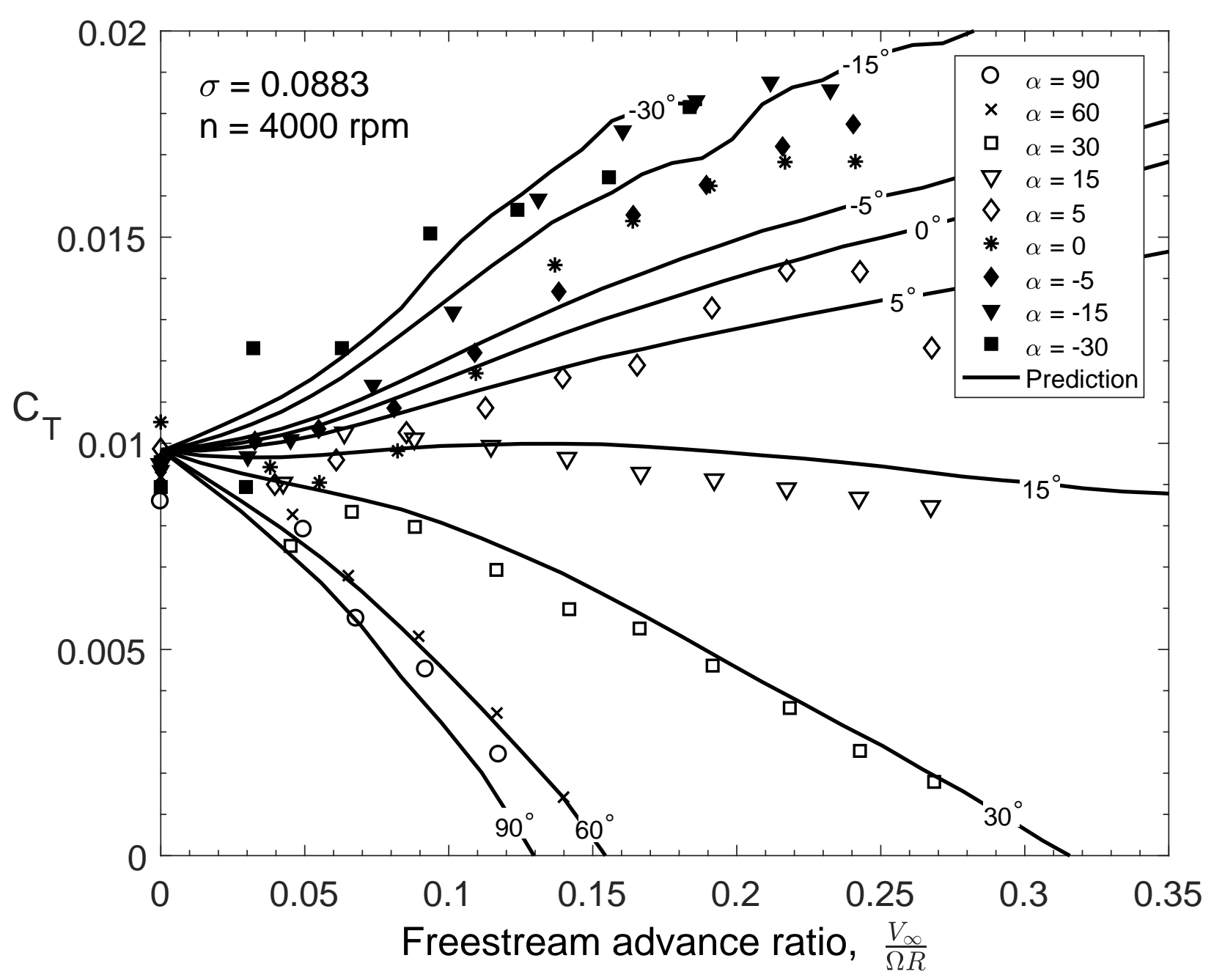

Figure 5.3: Thrust coefficient predictions versus advance ratio for the T-motor $18 \times 6.1$ for several angles of attack, $\mathrm{RPM}=4000$. 


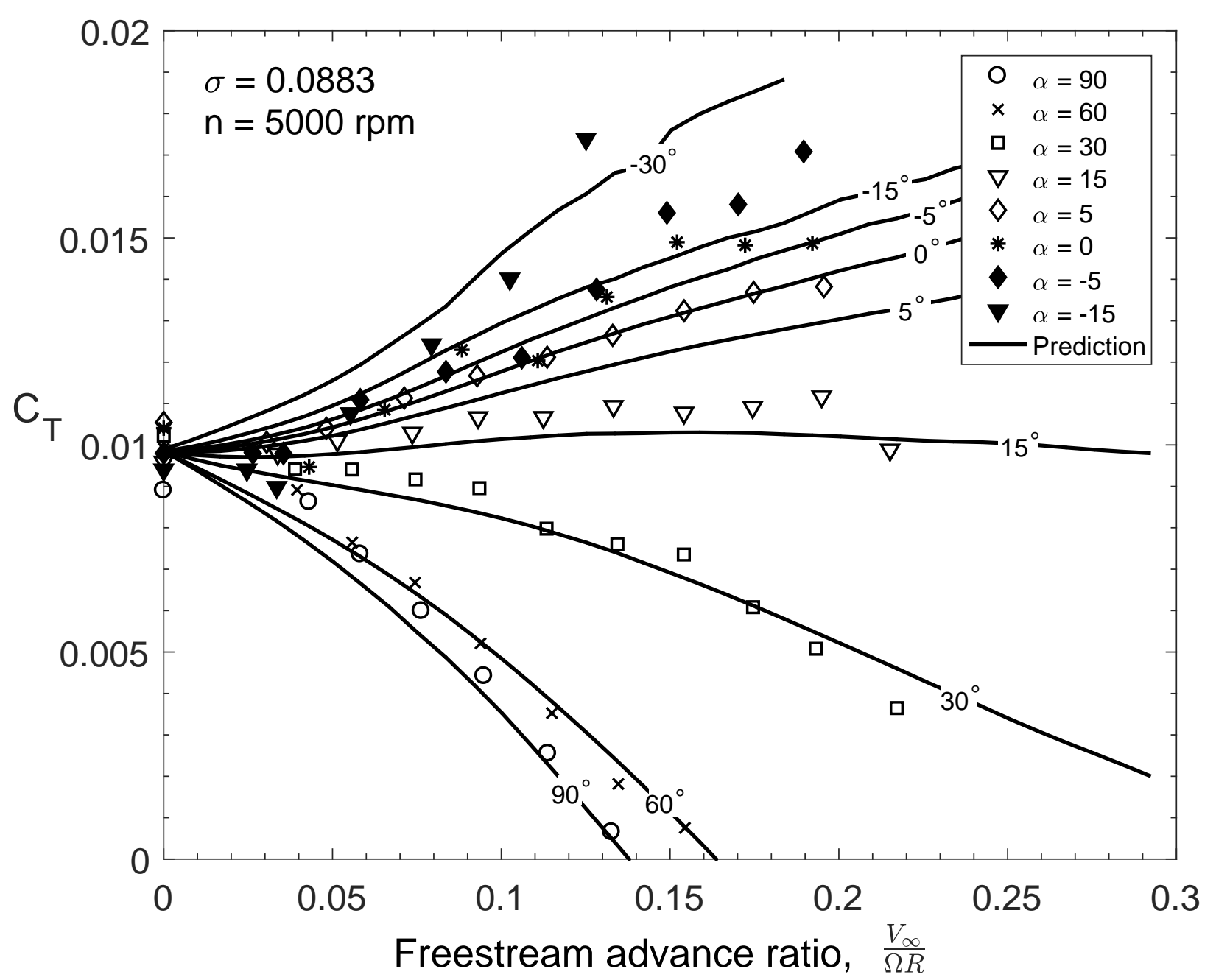

Figure 5.4: Thrust coefficient predictions versus advance ratio for the T-motor $18 \times 6.1$ for several angles of attack, RPM $=5000$. 
The power predictions, which are shown in Figs.5.5 through 5.7, are approximately 10-15\% less the measured power, and so a better comparison is made when organized by inflow angle. These are shown in Figs.5.8 through 5.10 for the angles of attack that encompass the operating conditions of a multirotor sUAV in cruise $\left(0^{\circ}\right.$ to $\left.90^{\circ}\right)$. These correspond to flight conditions which are temporary encountered by the vehicle, such as an air braking manoeuvre and are not sustained modes of flight. 


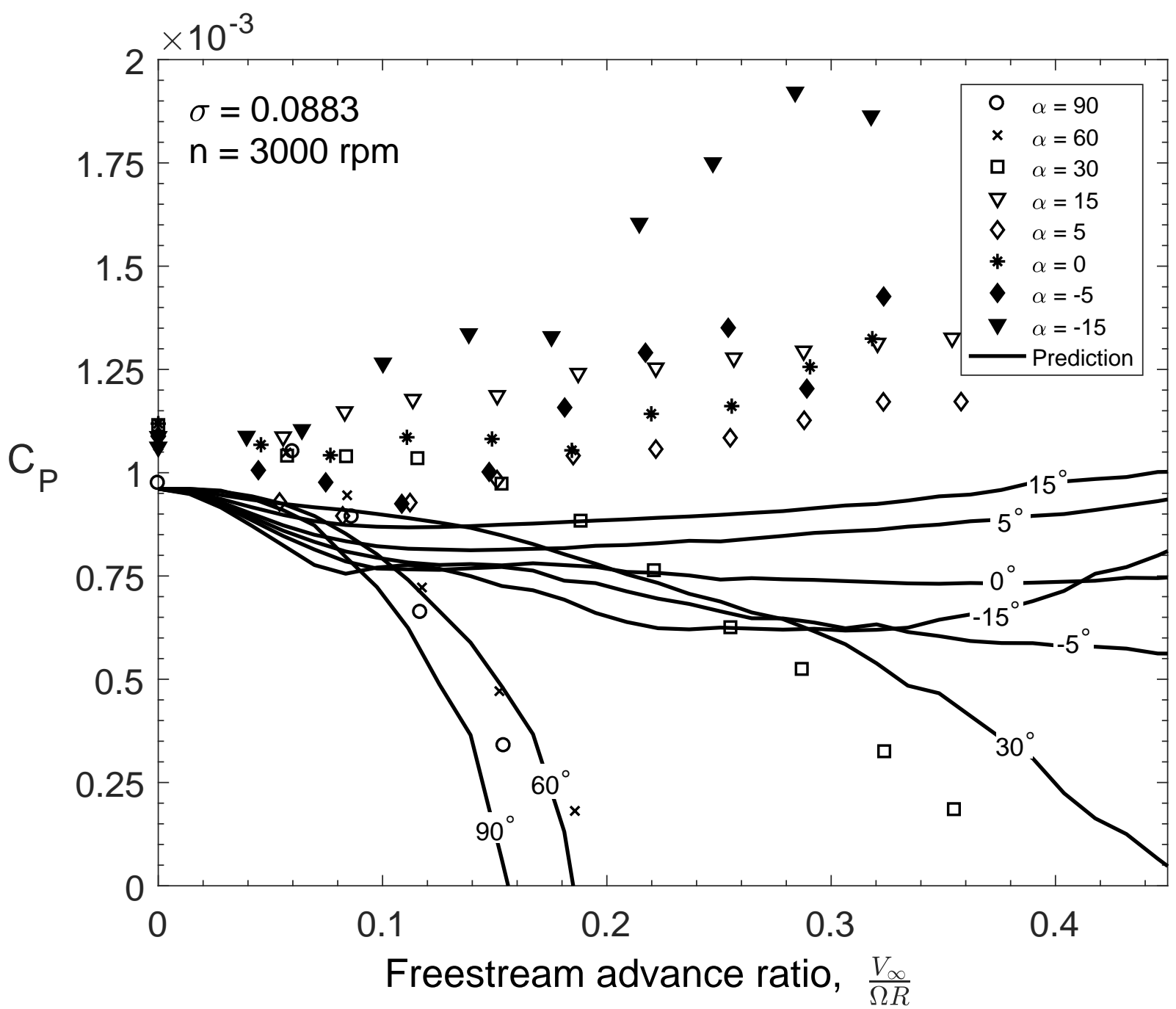

Figure 5.5: Power coefficient predictions versus advance ratio for the T-motor 18x6.1 for several angles of attack, $\mathrm{RPM}=3000$. 


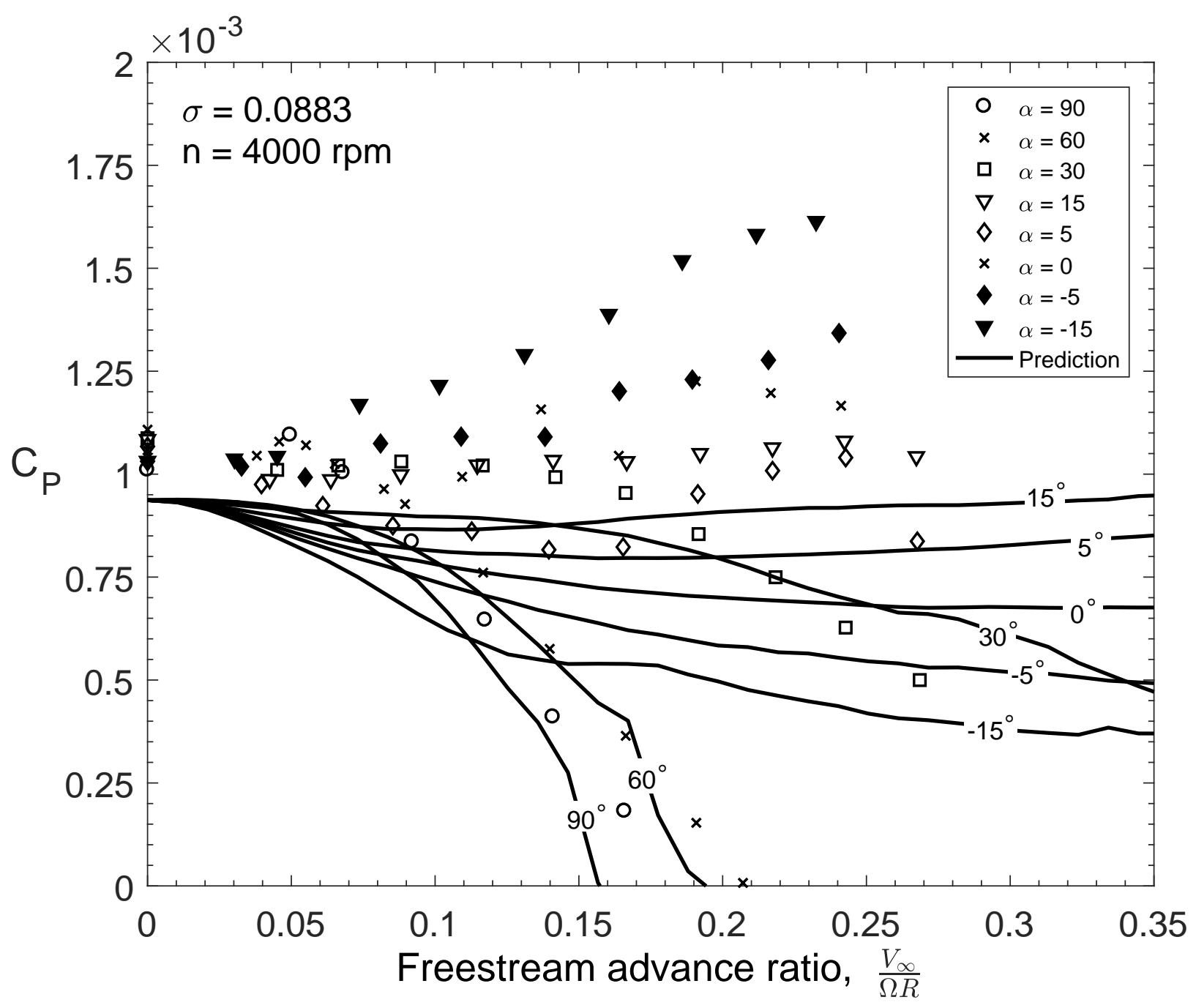

Figure 5.6: Power coefficient predictions versus advance ratio for the T-motor $18 \times 6.1$ for several angles of attack, $\mathrm{RPM}=4000$. 


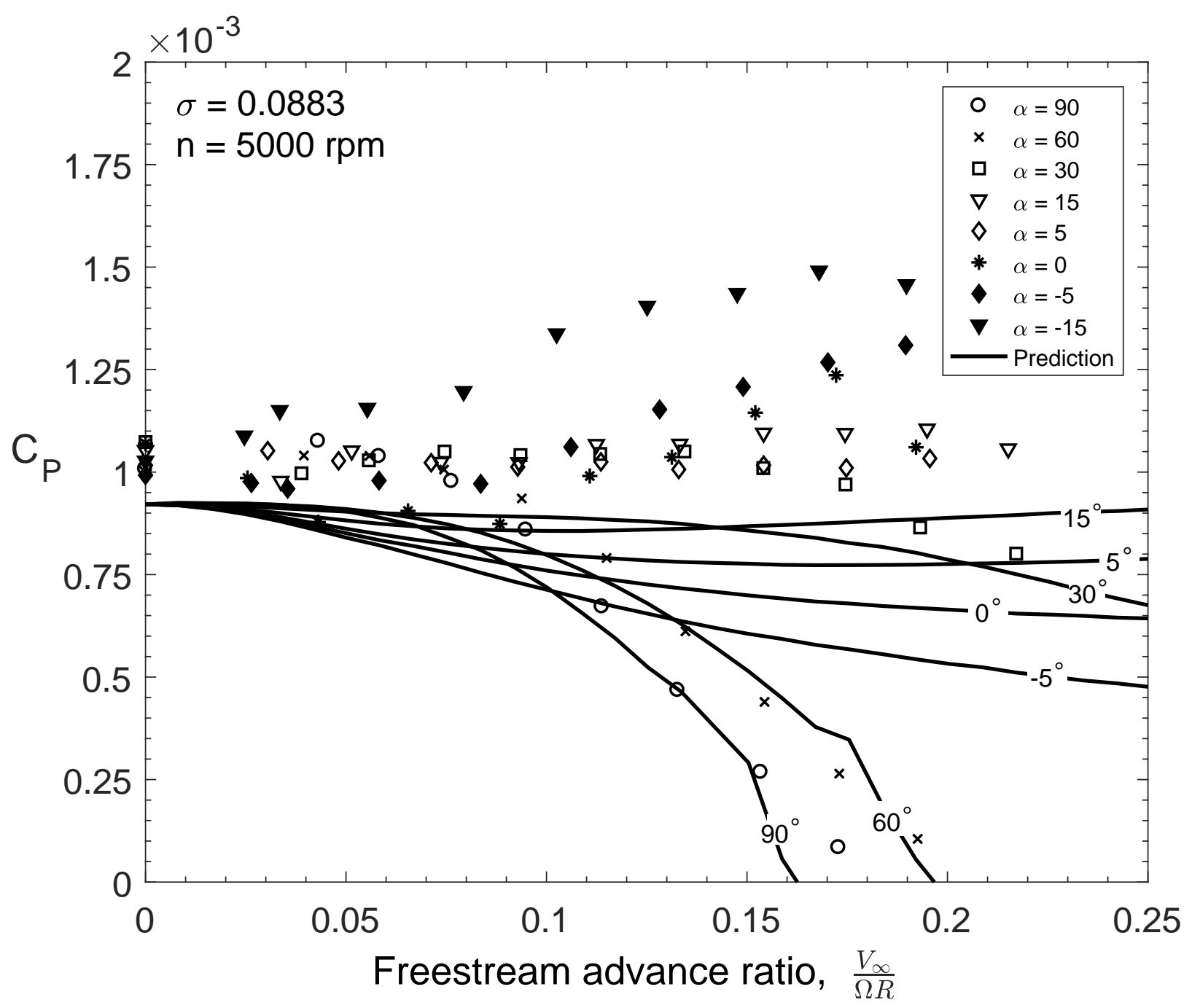

Figure 5.7: Power coefficient predictions versus advance ratio for the T-motor $18 \times 6.1$ for several angles of attack, RPM $=5000$. 
In Figs.5.8 through 5.10, similar trends are observed between predictions and experimental measurements for inflow angles greater than $0^{\circ}$. Part of this difference is attributed to the experimental sources of error discussed in Sec.4.3, particularly resulting from the over-measurement of power from the test stand.

The $90^{\circ}$ and $60^{\circ}$ angles of attack predictions agree within $15 \%$ of the measured power until windmilling. For the $60^{\circ}$ case, the less-smooth power predictions represent the increasing contribution of profile power to the total power as an increasing large portion of the blade stalls. This is evident for the $30^{\circ}$ case, shown in Fig.5.9, where there is a cross-over point between the measured and predicted results due to an over-prediction in profile power. A possible explanation for this behaviour is that stall on portions of the blade is partially delayed, in part due to the radial velocity component. This is shown with the induced power predictions in Fig.5.11, which predict the blade stalling at an advance ratio of 0.31 . This is also shown for the $15^{\circ}$ and $5^{\circ}$ predictions, which deviate an additional 13\%, from the initial $20 \%$ offset, at an advance ratio of 0.36 .

For the $0^{\circ}$ inflow angle, the power predictions begin to diverge from the experimental measurements at advance ratios above 0.1. For these cases, the more complicated inflow distributions are not captured using momentum theory. In such cases, the inflow is on the aft portion of the rotor disk is affected by the tip vortices shed from the leading portion of the rotor. It is well known that momentum theory is invalid for $-2 \leq \frac{V_{\infty} \sin \alpha}{v_{h}} \leq 0$ [13], or when using the freestream advance ratio, $-2 \leq \frac{\mu_{\infty} \sin \alpha}{\left(\sqrt{\frac{C_{T}}{2}}\right)} \leq 0$, though for the $\alpha=0^{\circ}$ case, the upper limit appears to be approximately at $\mu=0.1$. 


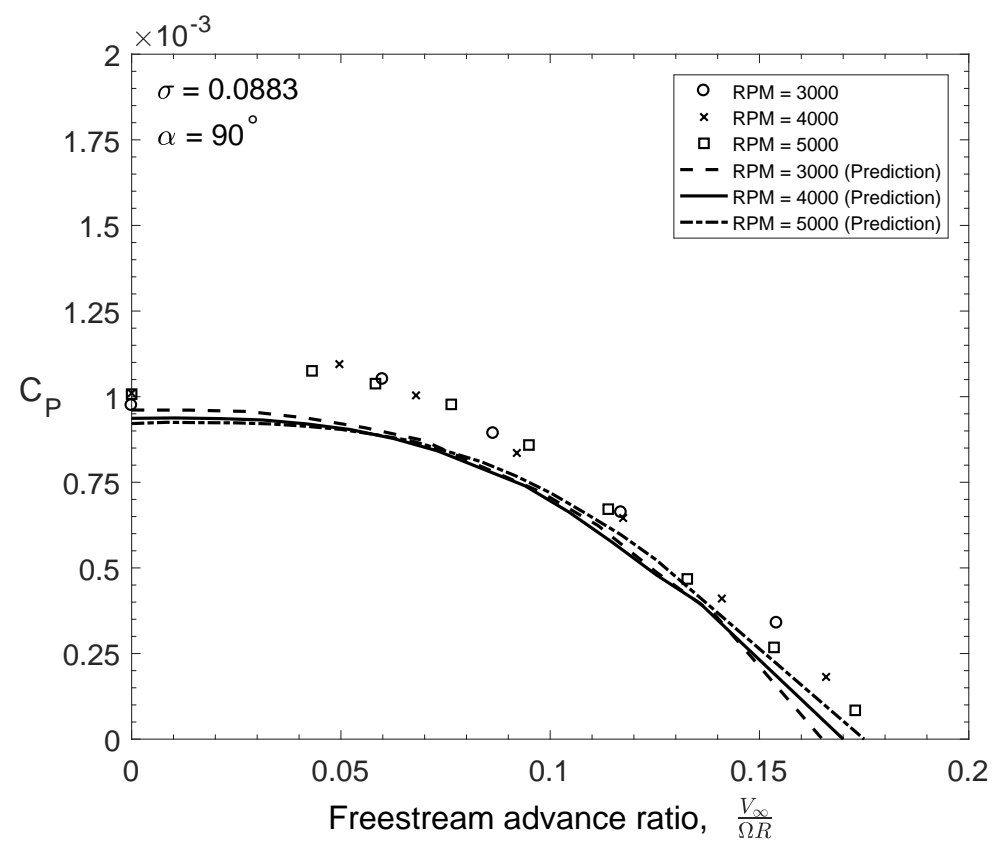

(a) $\alpha=90^{\circ}$.

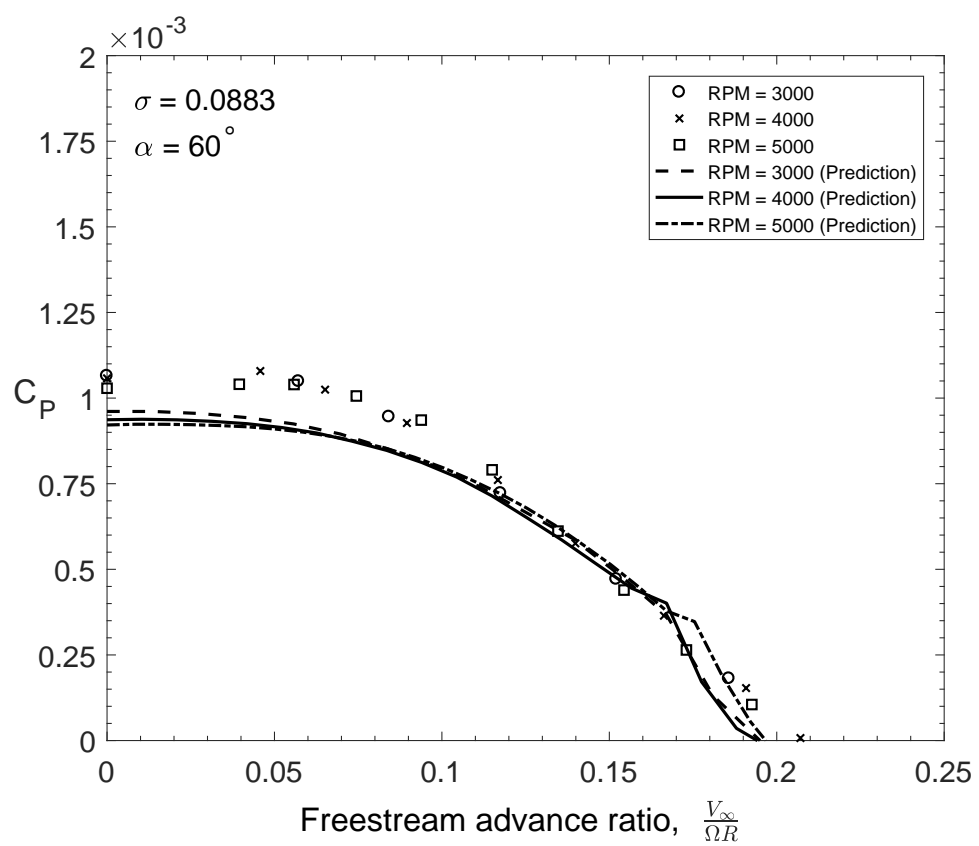

(b) $\alpha=60^{\circ}$.

Figure 5.8: Power predictions versus advance ratio for the T-motor $18 \times 6.1$ for $\alpha=90^{\circ}$ and $\alpha=60^{\circ}$. 


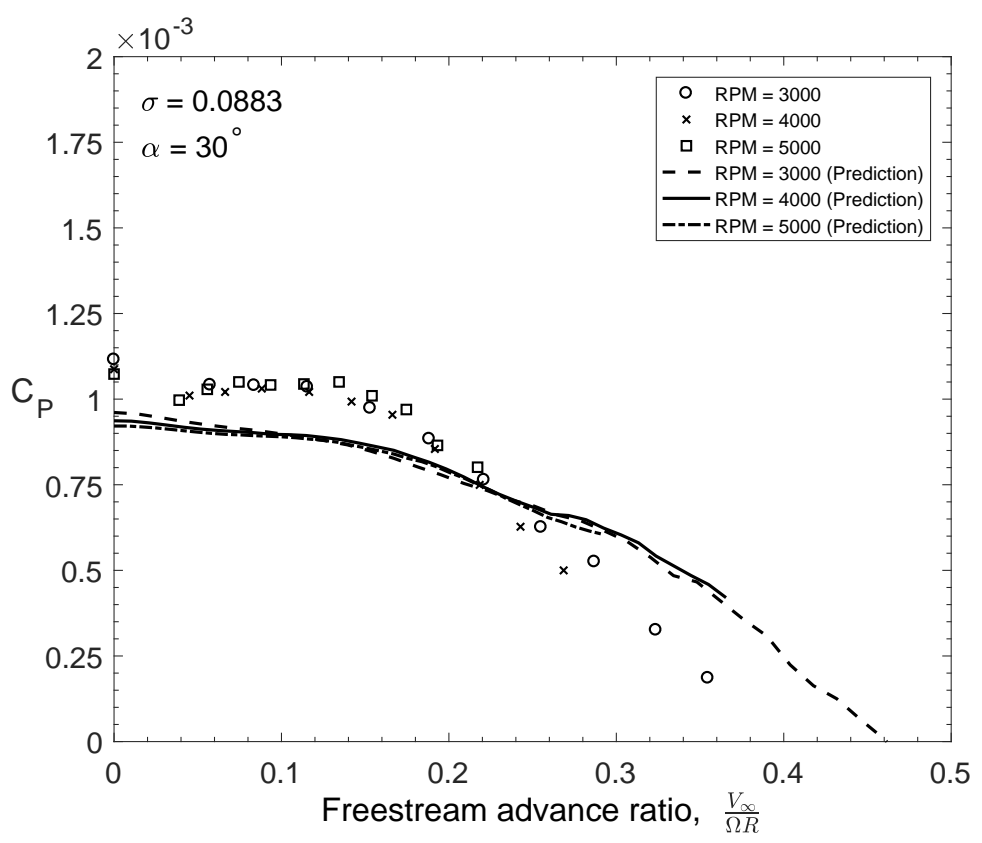

(a) $\alpha=30^{\circ}$.

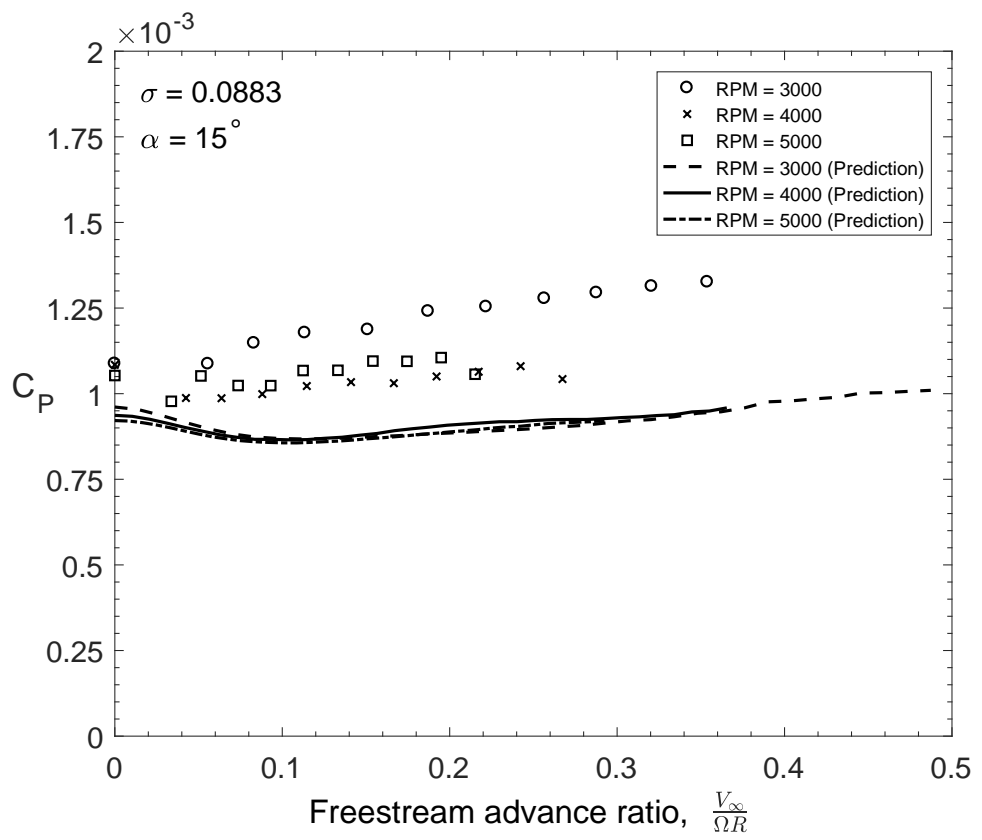

(b) $\alpha=15^{\circ}$.

Figure 5.9: Power predictions versus advance ratio for the T-motor $18 \times 6.1$ for $\alpha=30^{\circ}$ and $\alpha=15^{\circ}$. 


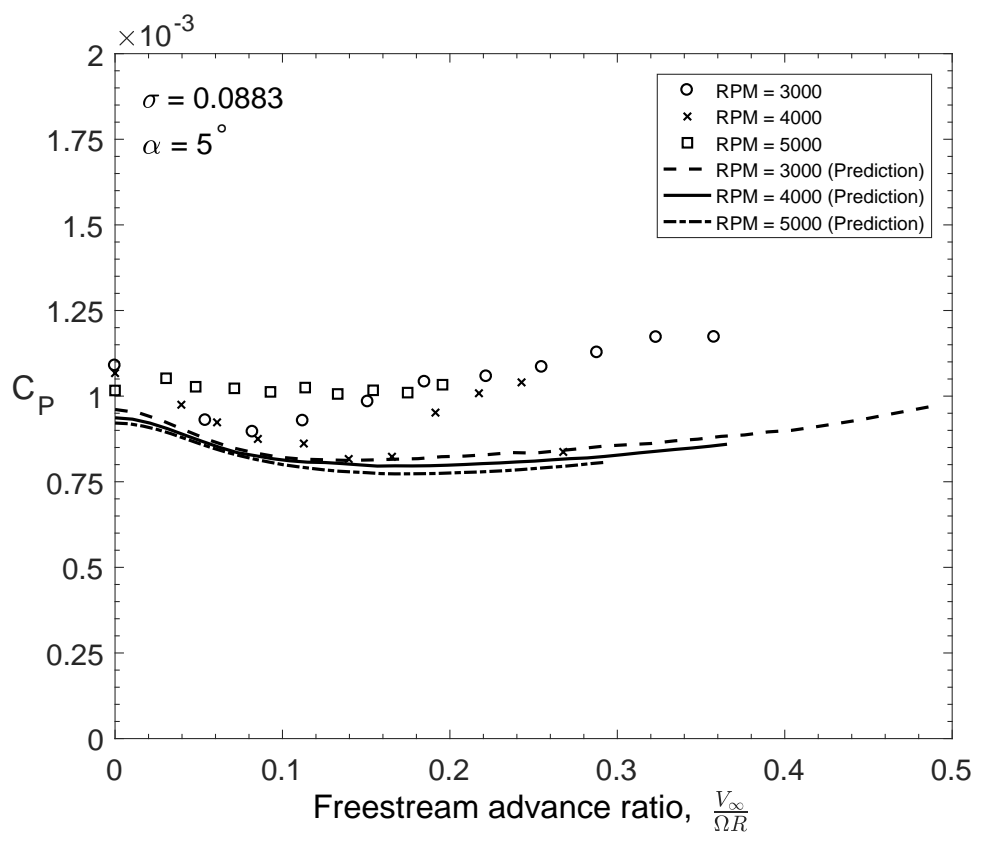

(a) $\alpha=5^{\circ}$.

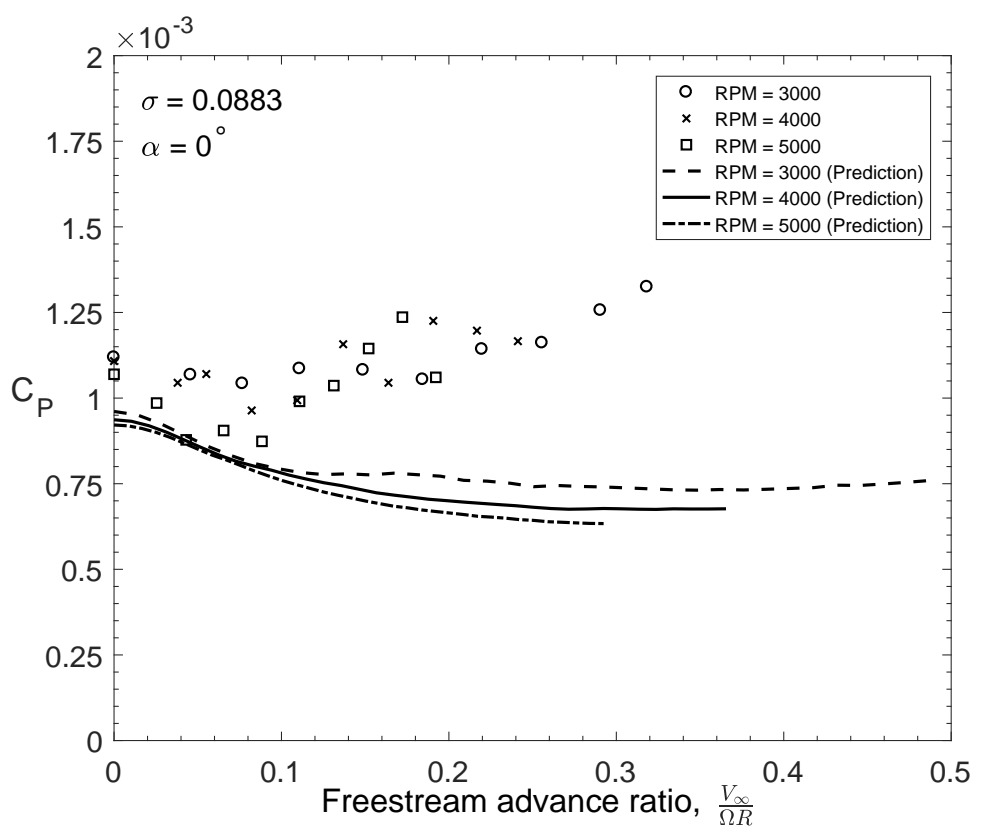

(b) $\alpha=0^{\circ}$.

Figure 5.10: Power predictions versus advance ratio for the T-motor $18 \times 6.1$ for $\alpha=5^{\circ}$ and $\alpha=0^{\circ}$. 


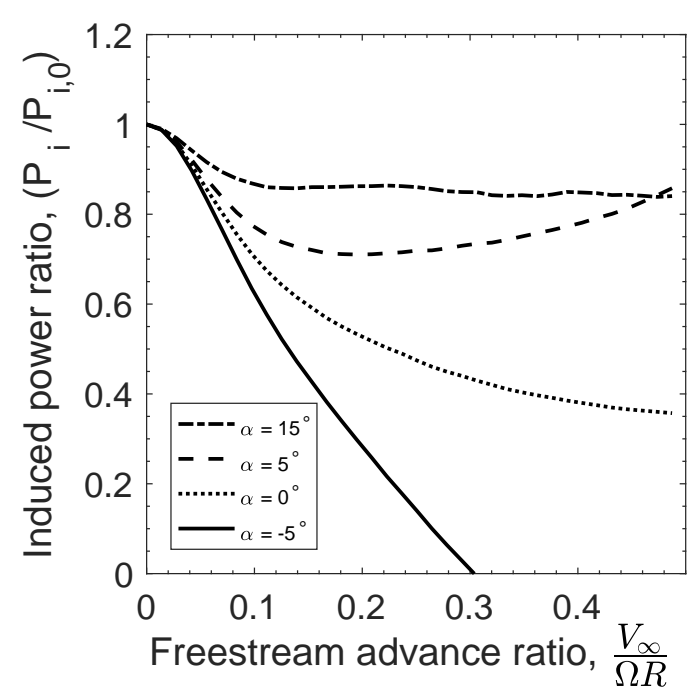

(a)

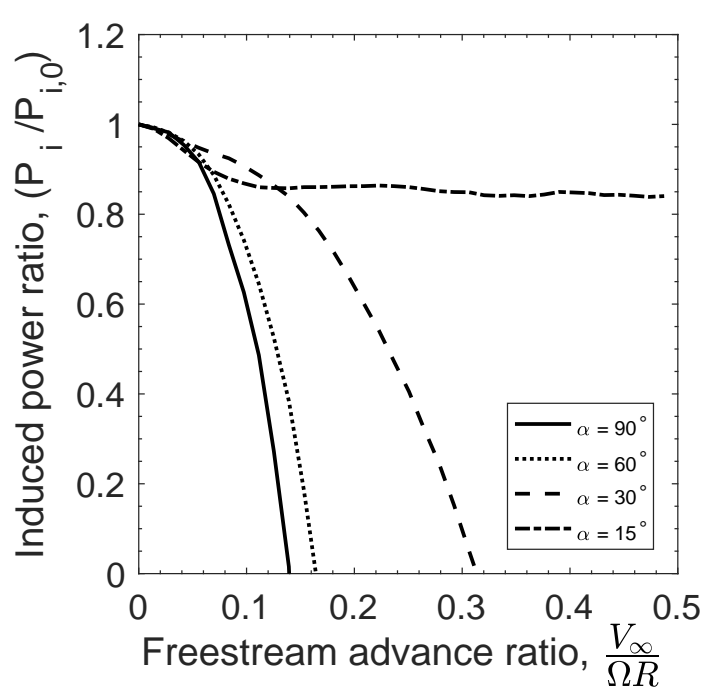

(b)

Figure 5.11: Induced power ratio versus advance ratio in forward flight.

\subsubsection{Azimuth Sensitivity}

The influence of the number of azimuth positions have on the predicted results is shown in Fig.5.12 for several advance ratios. The plot shows the percent difference in thrust coefficient compared with cases using up to 360 azimuth positions. For the advance ratios indicated, a percentage difference of less than $1.1 \%$ when 8 or more stations are used. Thus, based on these results, the predictions in this thesis were performed using 8 azimuth stations. 


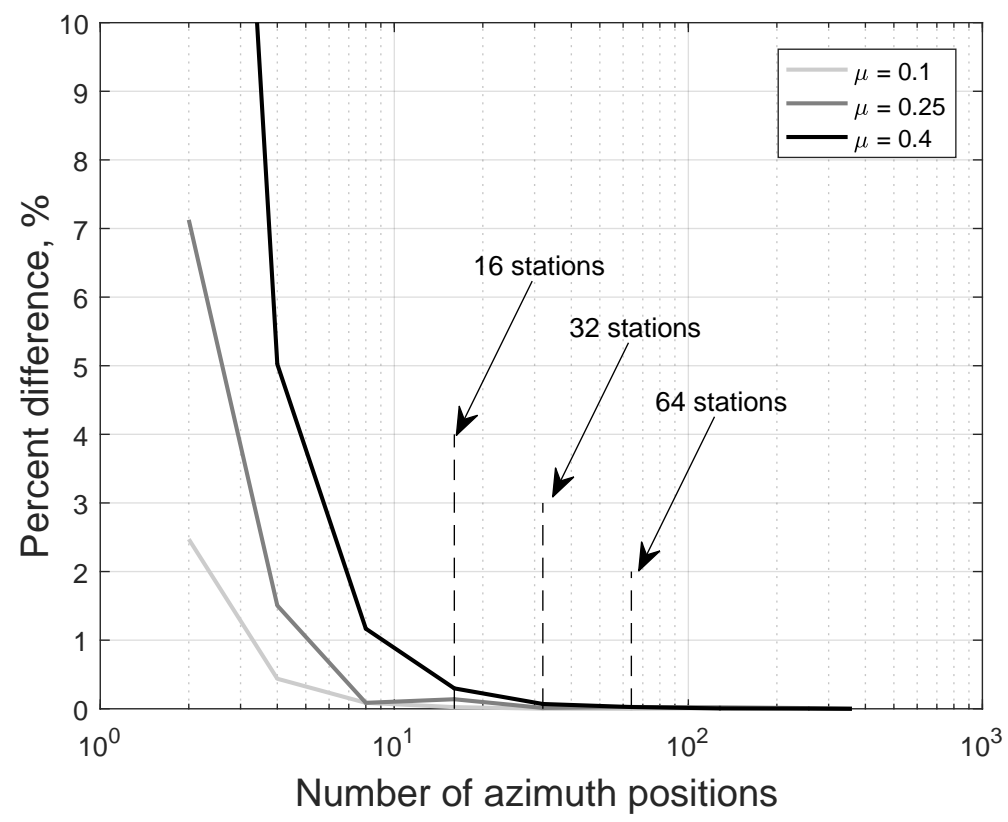

Figure 5.12: Azimuth station convergence behaviour on the thrust coefficient.

\subsection{Rotor Performance Model Verification}

To verify that the prediction model, in addition to capturing the rotor thrust and power, captures some of the aerodynamic effects described in Sec.3.3, the sectional aerodynamics along the blade are investigated in detail for several advance ratios.

\subsubsection{The Rotor Inflow Variation Due to Retreating/Advancing Blade Effects}

The motion of the retreating and advancing blade has the effect of changing the angle of attack distribution along the blade and results in the tilting of the lift vector, which is most pronounced on the retreating side. This creates a roll moment about the rotor hub and has a large impact on the cyclical torque. For large advance ratios, the inboard portion of the retreating blade may see no relative velocity or be operating close to reverse flow, which is when the section's trailing edge is pointed towards the freestream. Figures 5.13a and 5.13b depict the flow environment when the tangential velocity is zero and reversed, respectively. In these extreme 
cases, typically on the inboard blade, the sectional lift vector contributes to the torque and the drag vector is pointed in the direction of the thrust.

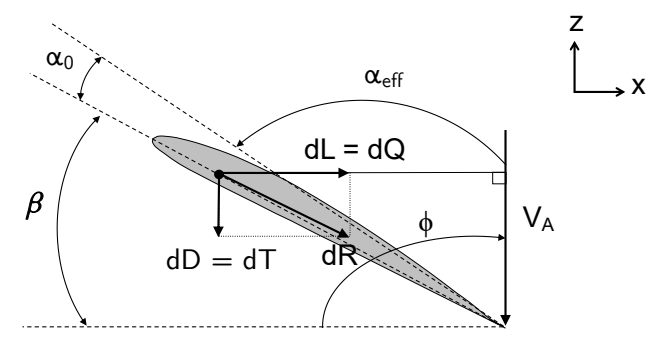

(a) $\phi=90$

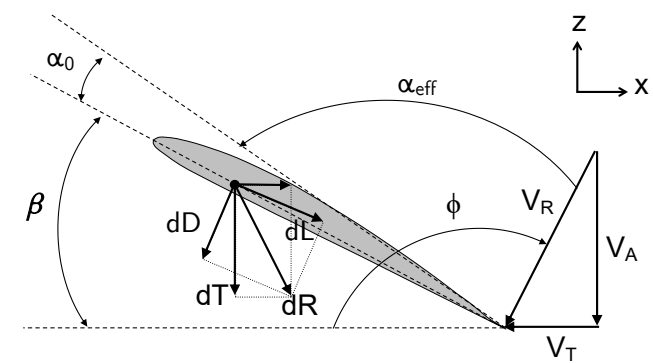

(b) $\phi>90$

Figure 5.13: Elemental flow environment in the reverse flow region.

The reverse flow region by definition is centred at $\phi=270^{\circ}$ and covers a circular area with diameter $\mu_{x}$. Figure 5.14 shows the inflow angle distribution for two advance ratios and shows where the local inflow angle is greater than $90^{\circ}$. For most rotors, $\mu_{x}=0.5$ is the limiting upper advance ratio since, as indicated in Fig.5.14b, a significant portion of the retreating blade is either completely reversed or is angled perpendicular to the inflow.

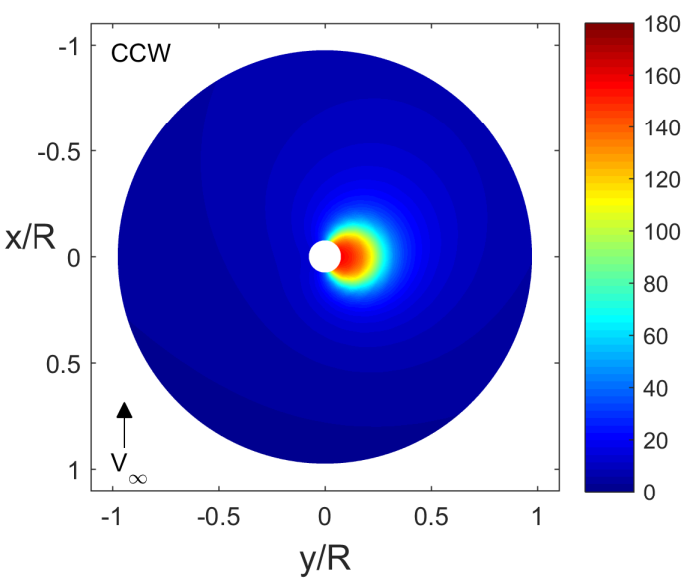

(a) $\mu_{x}=0.25\left(C_{T} / \sigma=0.1672\right)$.

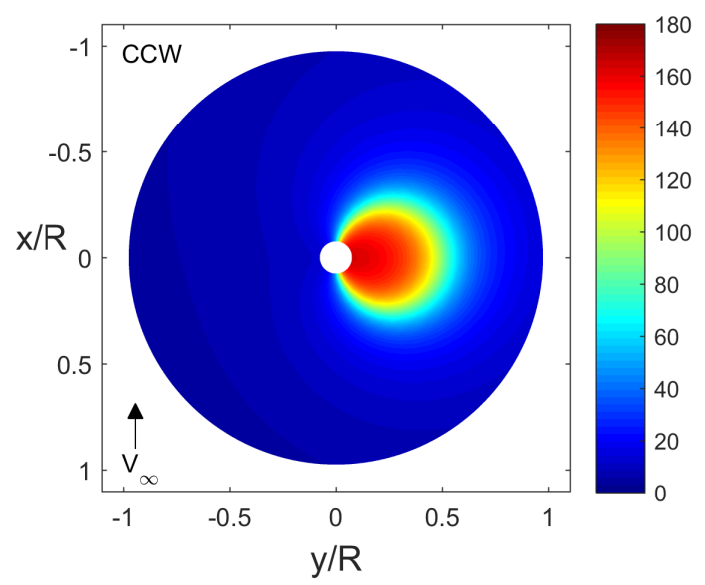

(b) $\mu_{x}=0.5\left(C_{T} / \sigma=0.1772\right)$.

Figure 5.14: Inflow angle, $\phi[\mathrm{deg}]$.

The effective angle of attack distribution is shown in Fig.5.15. For $\mu_{x}=0.25$, most of the 
blade sections, including those on the retreating side, are operating at positive angles of attack and are unstalled. For $\mu_{x}=0.5$, almost the entire retreating blade is operating at negative angles of attack, which is a consequence of the blade having a fixed pitch, and thus, maintaining level flight requires the reactionary trim from multiple rotors.

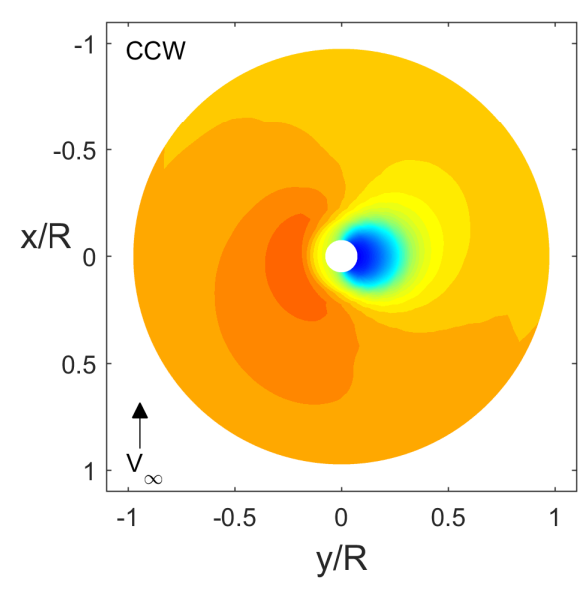

(a) $\mu_{x}=0.25\left(C_{T} / \sigma=0.1672\right)$.

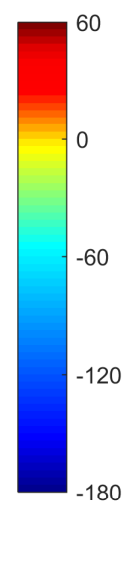

Figure 5.15: Effective angle of attack, $\alpha_{\text {eff }}[\mathrm{deg}]$.

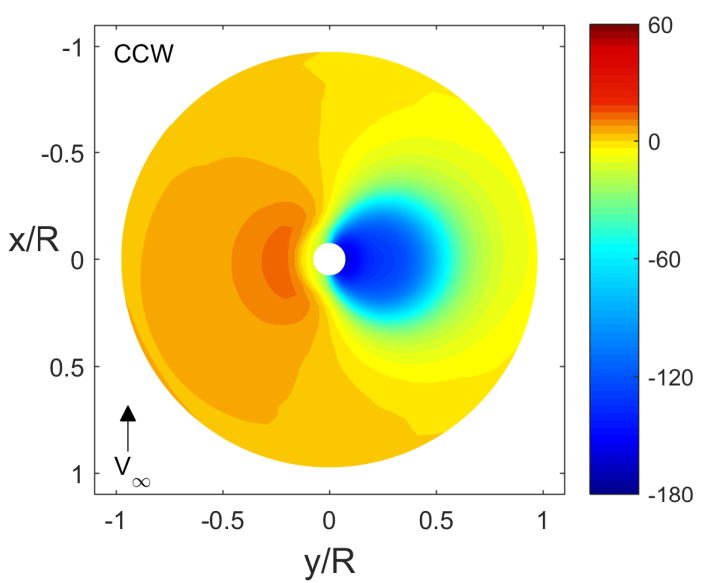

(b) $\mu_{x}=0.5\left(C_{T} / \sigma=0.1772\right)$.

\subsubsection{Sectional Lift and Drag Coefficients}

The lift coefficient distribution, shown in Fig.5.16, shows that even for the $\mu_{x}=0.25$ case, the portion of the retreating blade within the reverse flow region produces downward lift. The $\mu_{x}=0.5$ case shows that almost the entire retreating blade, not including the reverse flow region, produces downward lift. For both advance ratios, the lift coefficients inside the inner most portion of the reverse flow region are actually positive and in some cases are unstalled. This represents the cases where the section's trailing edge is pointing almost directly towards the flow. 


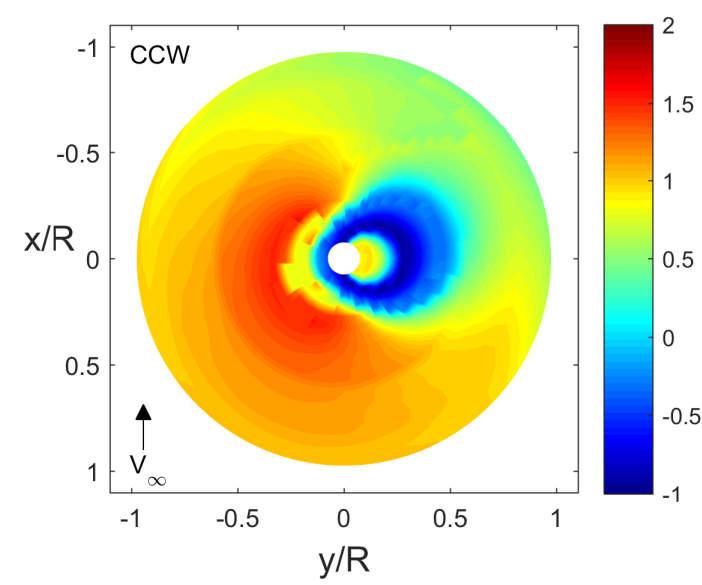

(a) $\mu_{x}=0.25\left(C_{T} / \sigma=0.1672\right)$.

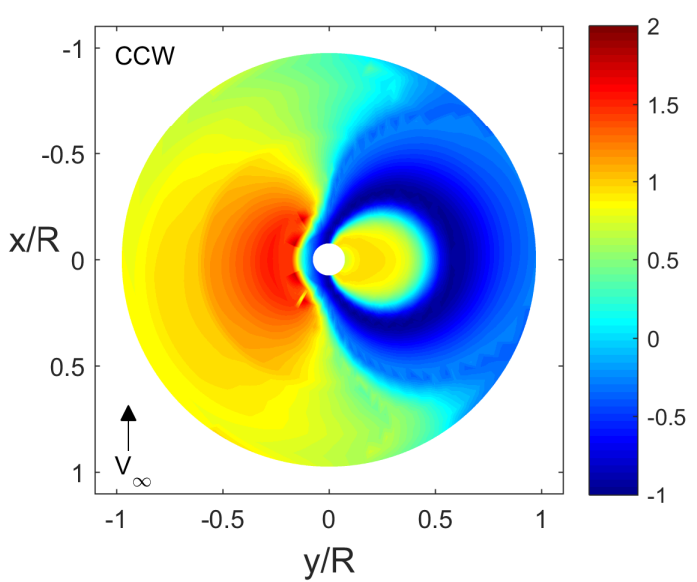

(b) $\mu_{x}=0.5\left(C_{T} / \sigma=0.1772\right)$.

Figure 5.16: Section lift coefficient, $c_{l}$.

Referring to Fig.5.17, the drag coefficients are highest in the regions that coincide with the areas in which the local inflow is perpendicular to the section, as expected. For $\mu_{x}=0.25$, a portion of the inboard region on the advancing side also has a higher drag coefficient since these sections are stalled.

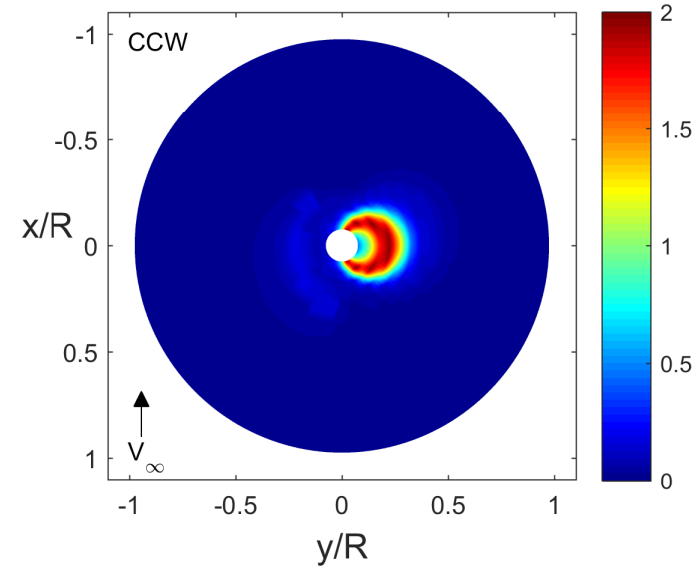

(a) $\mu_{x}=0.25\left(C_{T} / \sigma=0.1672\right)$.

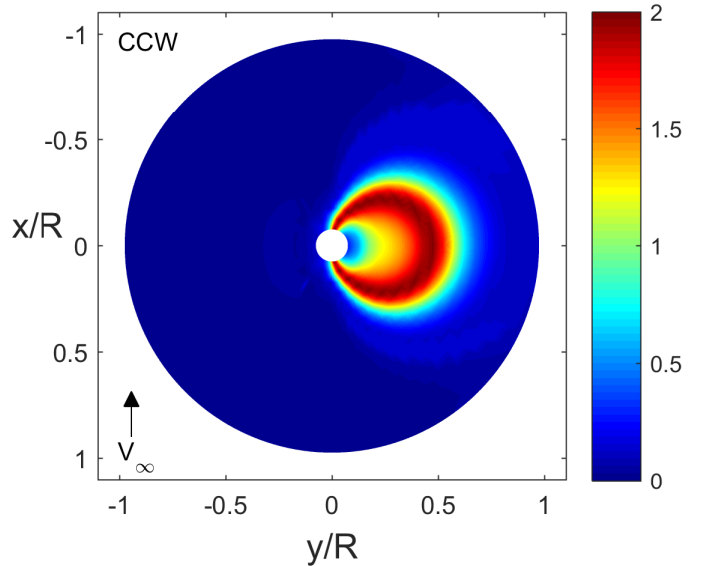

(b) $\mu_{x}=0.5\left(C_{T} / \sigma=0.1772\right)$.

Figure 5.17: Section drag coefficient, $c_{d}$. 


\subsubsection{Drag Divergence Mach Number and Compressibility}

On full scale rotor blades, drag divergence and compressibility effects have a considerable influence on rotor performance because the blade tip Mach numbers often approach the supersonic range. Large tip speeds come with two distinct characteristics; the advancing blade tips temporarily exceed the sound barrier, and the drag divergence threshold is exceeded for a portion of the blade. Reference 13 suggests that the onset of drag divergence occurs at a sectional Mach numbers of $M_{\mathrm{dd}}=0.85$. To determine whether the tips of the tested small rotor blades operate near the drag divergence range, the sectional Mach numbers are shown in Fig.5.18. Fig.5.18b shows that the maximum sectional Mach number observed for $\mu_{x}=0.5$ is slightly over 0.41 and less than $M_{\mathrm{dd}}$. Thus, the drag divergence threshold is not a limiting factor for the rotors investigated in this thesis.

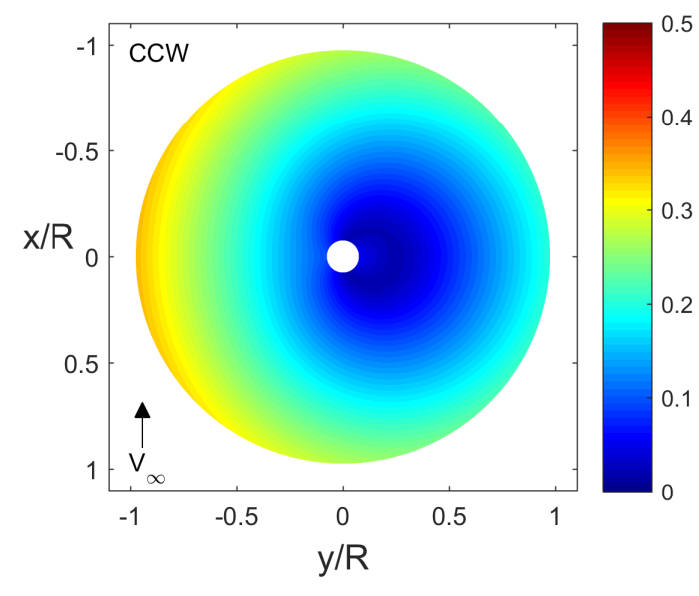

(a) $\mu_{x}=0.25\left(C_{T} / \sigma=0.1672\right)$.

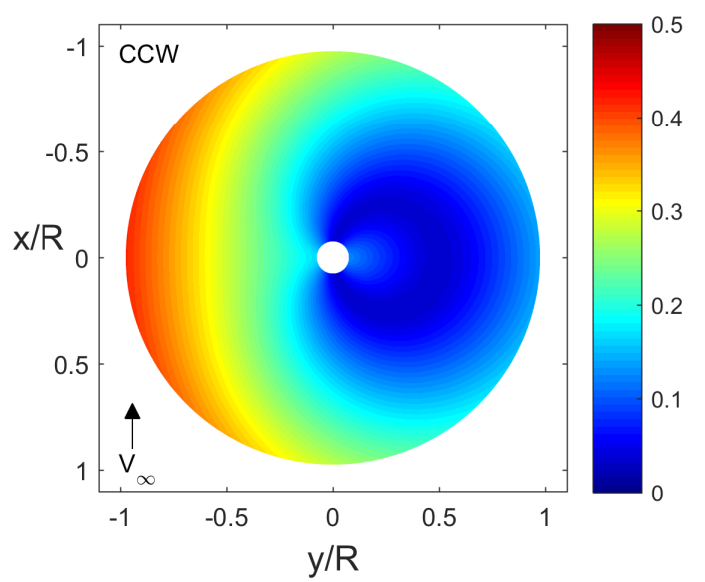

(b) $\mu_{x}=0.5\left(C_{T} / \sigma=0.1772\right)$.

Figure 5.18: Section Mach number, $M$.

\subsubsection{Thrust, Power and Induced Drag Distributions}

The thrust, power and induced drag distributions are shown in Figs.5.19 through 5.21. For both advance ratios, there is an observable reduction in thrust at the tips which is due to the local decrease in sectional chord and the imposing of the zero tip-thrust condition. Compared to full-scale rotor blades, a considerable amount of the thrust on the advancing side is produced 
by the inboard blade, starting at approximately $0.25 \mathrm{R}$, and is due to the corresponding large sectional chord and pitch angles of these sections.

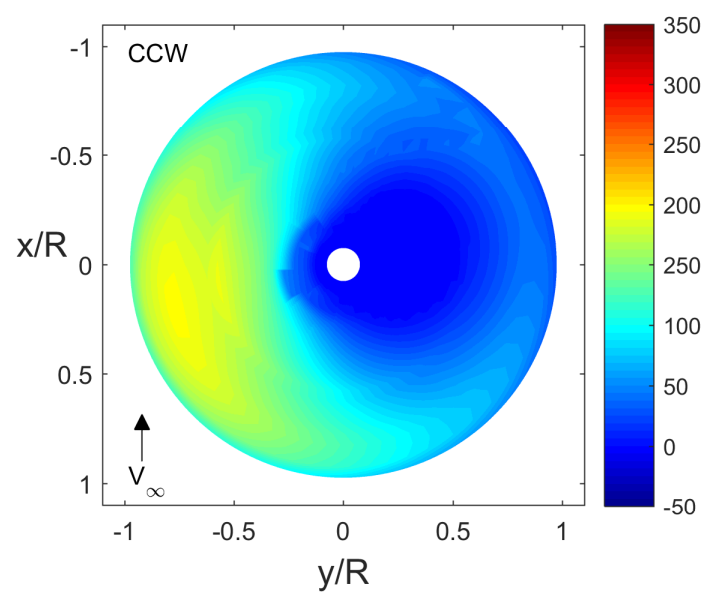

(a) $\mu_{x}=0.25\left(C_{T} / \sigma=0.1672\right)$.

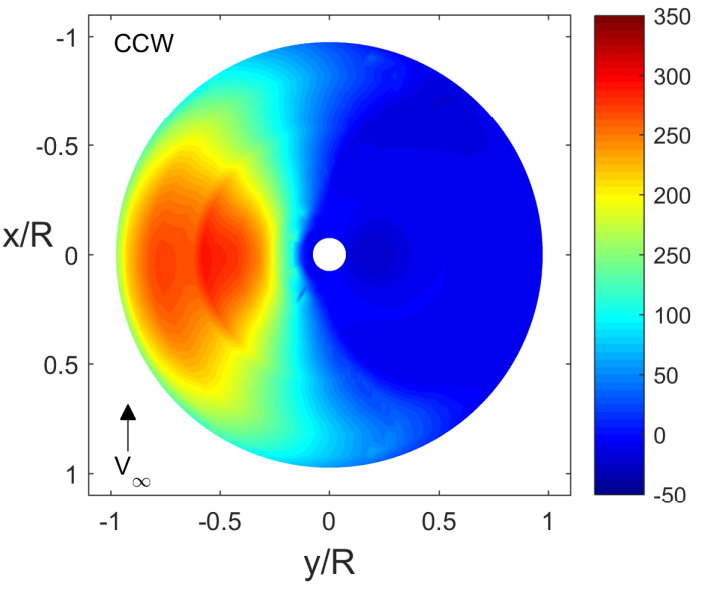

(b) $\mu_{x}=0.5\left(C_{T} / \sigma=0.1772\right)$.

Figure 5.19: Section thrust per unit span, $\frac{d T}{d r}\left[\frac{N}{m}\right]$.

Figure 5.20, shows the distributions of the total required power over the rotor plane. The power requirements on the retreating side are relatively low, compared to the advancing blade, due to the associated reduction in induced drag. This is also shown for the induced drag distributions in Fig.5.21. Interestingly, the inboard retreating blade for the $\mu_{x}=0.5$ advance ratio case has a localized increase in induced drag, coinciding with the reverse flow region, since the sections in this region are generating lift, despite being reversed. The maximum induced drag corresponds with the areas that produce the most thrust and have the largest pitch angle, and therefore corresponds with the inboard portion of the advancing blade. 


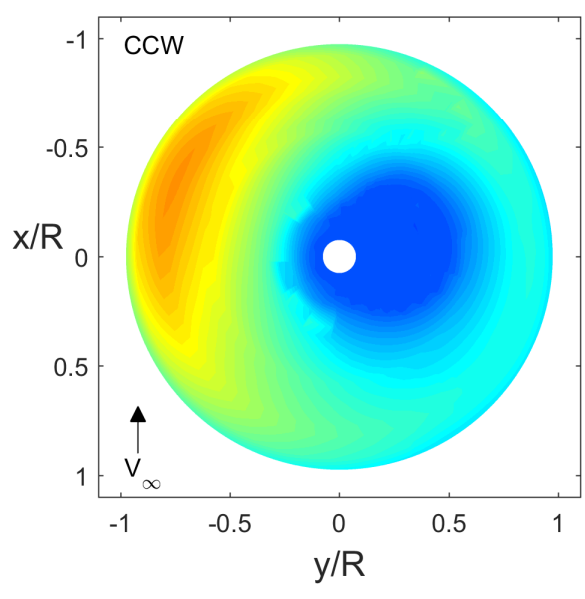

(a) $\mu_{x}=0.25\left(C_{T} / \sigma=0.1672\right)$.

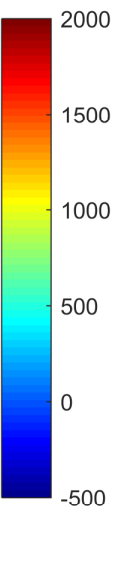

Figure 5.20: Section power per unit span, $\frac{d P}{d r}\left[\frac{W}{m}\right]$.

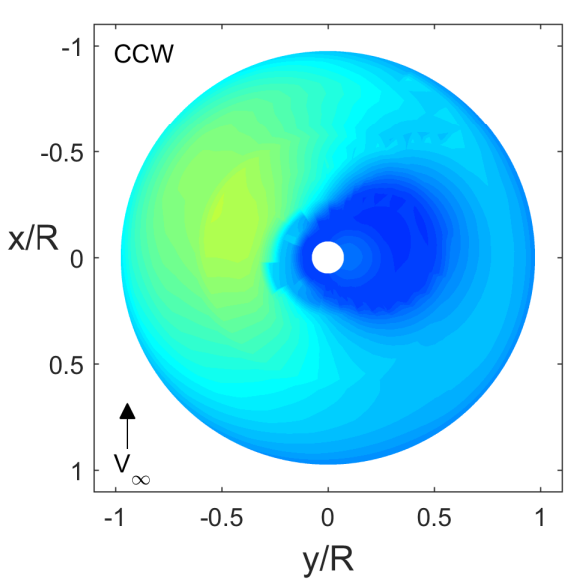

(a) $\mu_{x}=0.25\left(C_{T} / \sigma=0.1672\right)$.

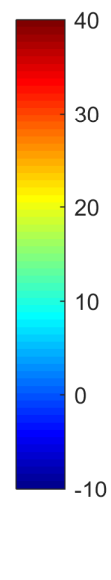

Figure 5.21: Section induced drag per unit span, $\frac{d D i}{d r}\left[\frac{N}{m}\right]$.

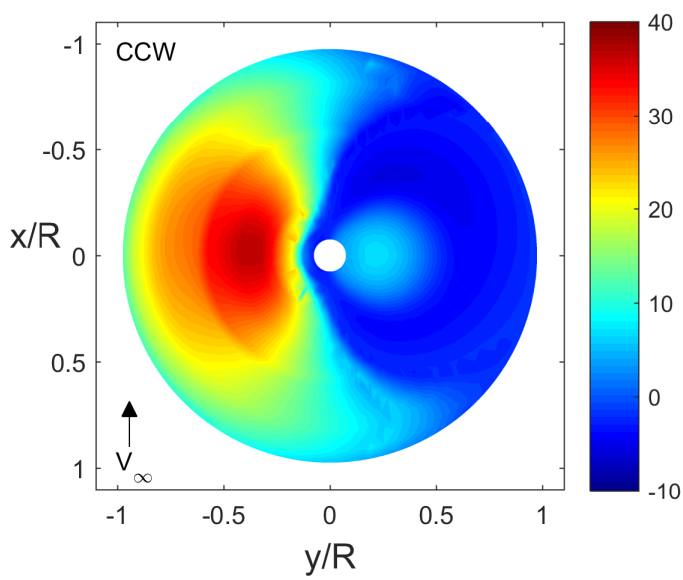

(b) $\mu_{x}=0.5\left(C_{T} / \sigma=0.1772\right)$.

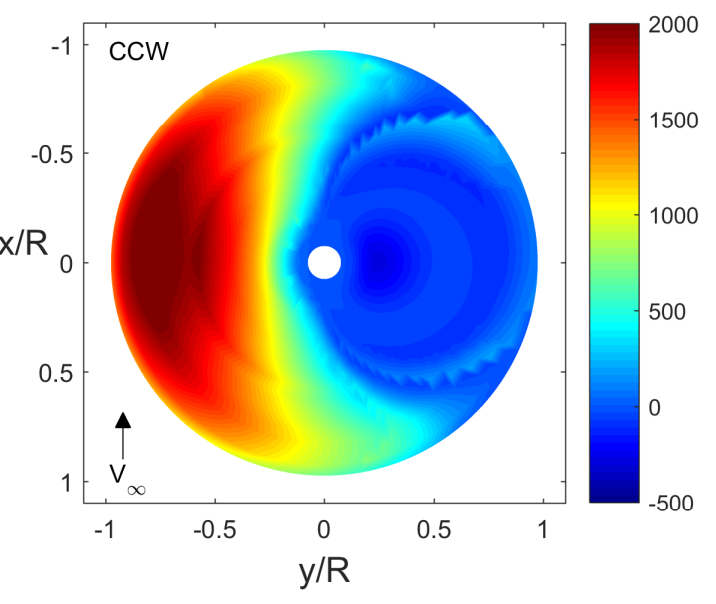

(b) $\mu_{x}=0.5\left(C_{T} / \sigma=0.1772\right)$. 
that determines the velocities induced by an infinite series of trailing ring vortices both at the the rotor plane and in the surrounding flow field. The model assumes that there are no mutual interactions between the wake elements themselves, and thus, it is a reasonable assumption that if the model simulates the flow field for a single rotor, it can be used to estimate the flow field of multiple rotors in configuration.

\subsubsection{Wake Length and Ring Segmentation Effects}

Determining the minimum number of rings and ring elements required to approximate the analytical solution is important for reducing the computational cost of the model. The performance of several wake geometries were compared to determine the influence that the number of ring wake elements and ring segments used has. Figures 5.22 and 5.23 show a comparison for the normal component of the induced velocity ratio at the rotor disk between the analytical solution and the various simulated cases.

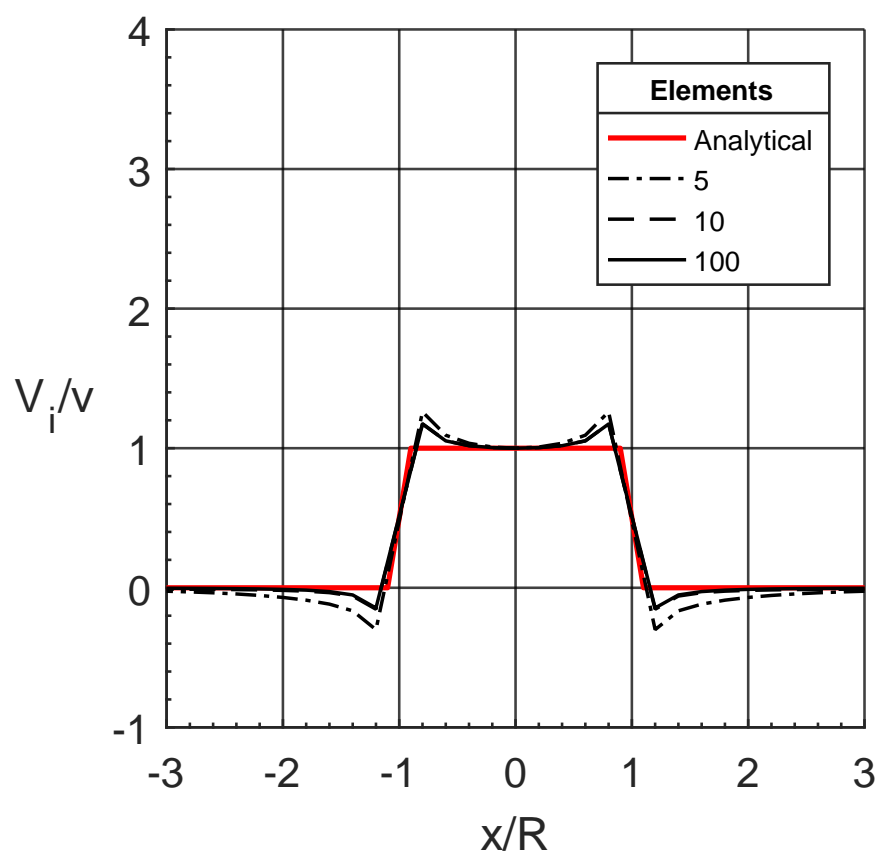

Figure 5.22: Normal component of the induced velocity ratio at the rotor plane for several ring wake elements (each having 64 vortex line segments). 
Figure 5.22 shows that the change in induced velocity ratio is relatively insensitive between the 10 and 100 ring cases. The shape of the ring elements, shown in Fig.5.23, has a more noticeable effect on the induced velocity ratio but becomes less influential beyond 16 segments. Therefore for a lightly loaded rotor, a minimum of 16 segments and 10 ring elements can be used with acceptable precision in hover. For the other flight conditions, using more than 50 rings elements is suggested.

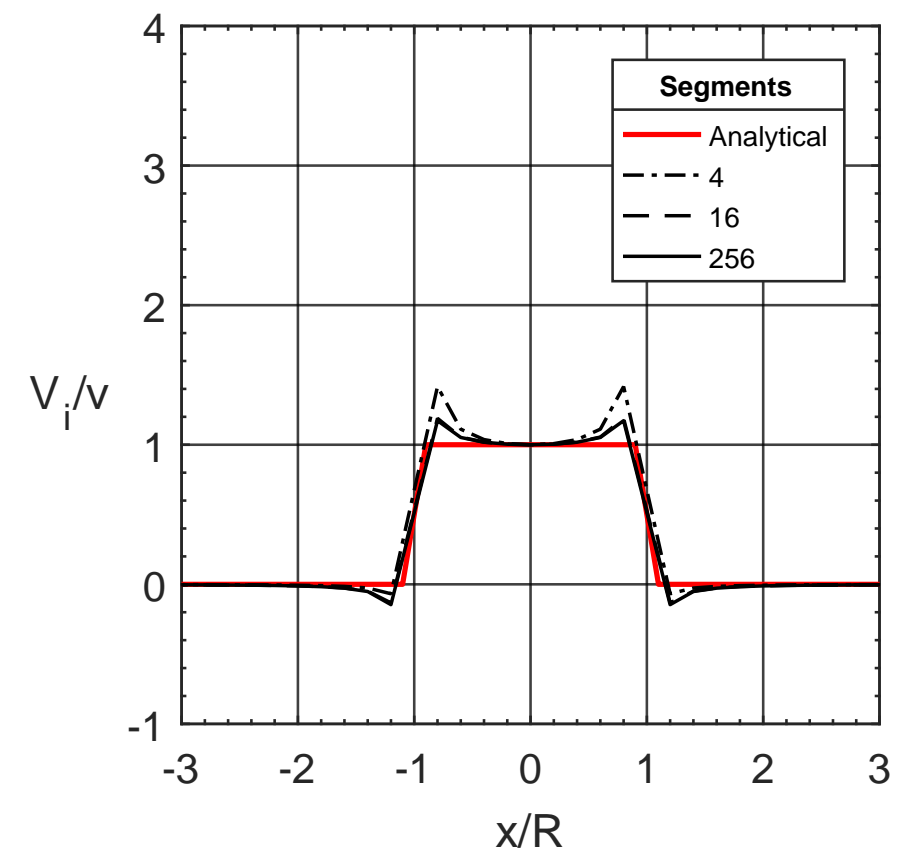

Figure 5.23: Normal component of the induced velocity ratio at the rotor plane for several ring segments (each wake consisting of 100 ring wake elements).

\subsubsection{Induced Velocities in Forward Flight}

The comparison between the vertical induced velocities obtained using the vortex wake method and the analytical result are shown in Figs 5.24 through 5.27 for several skew angles. For these predictions, 64 ring segments and 100 ring elements are used. The red lines represents the analytical solution and the black lines represent results generated from the wake model. 


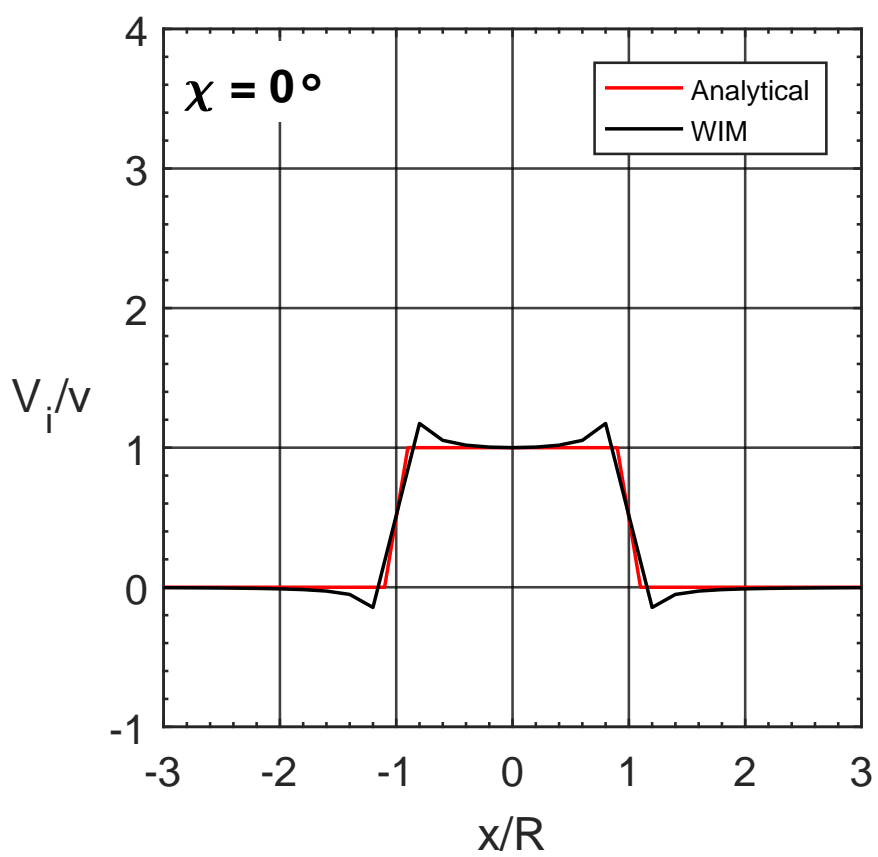

Figure 5.24: Induced velocity distributions along the rotor plane for $\chi=0^{\circ}$.

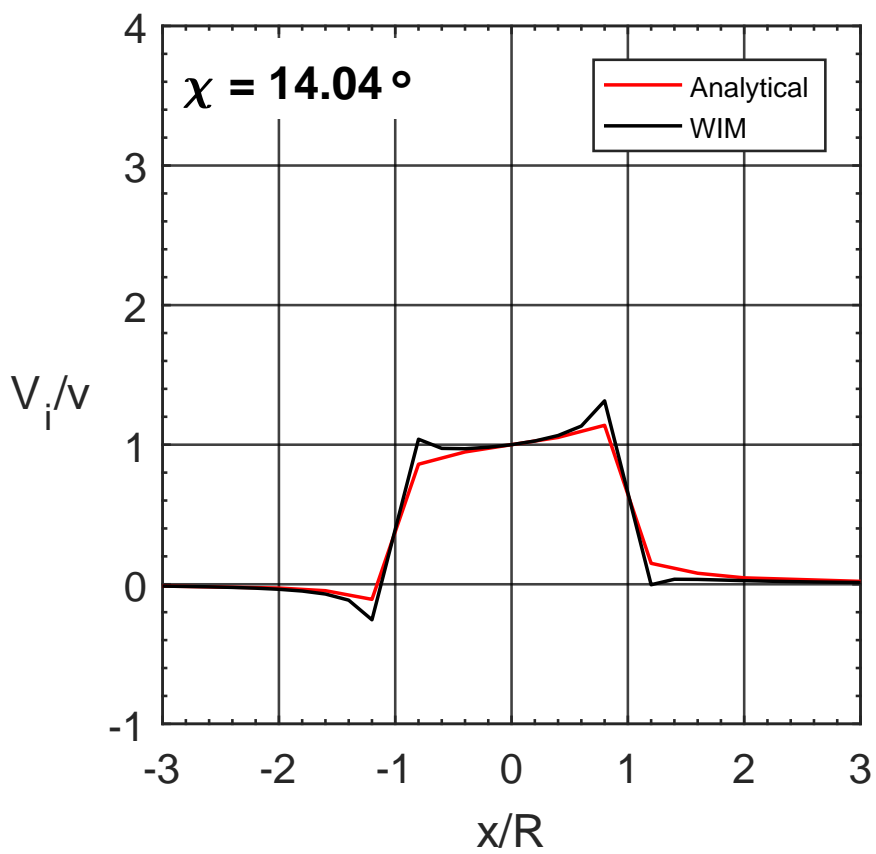

Figure 5.25: Induced velocity distributions along the rotor plane for $\chi=14.04^{\circ}$. 


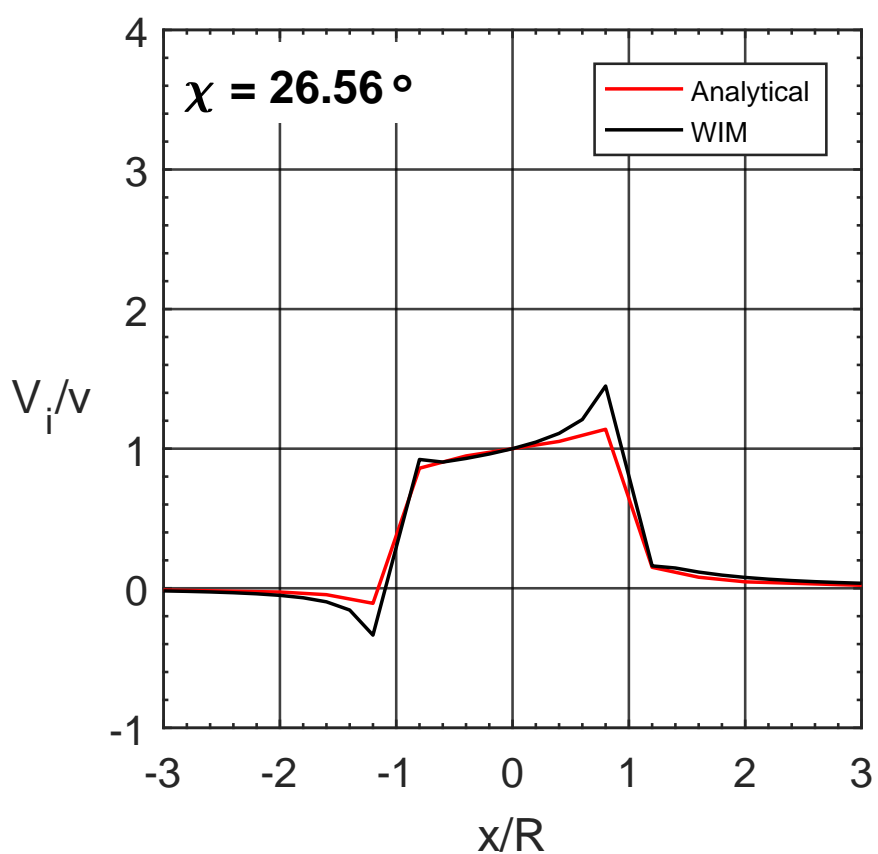

Figure 5.26: Induced velocity distributions along the rotor plane for $\chi=26.56^{\circ}$.

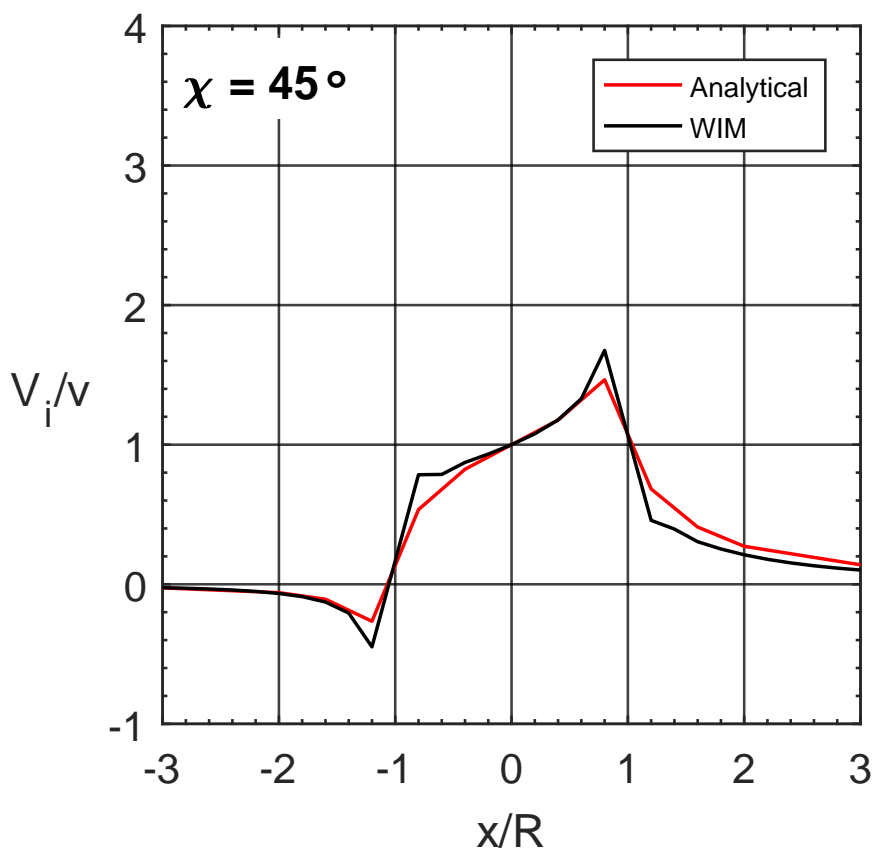

Figure 5.27: Induced velocity distributions along the rotor plane for $\chi=45^{\circ}$. 
The predictions from the vortex wake model agree with the analytical solution for the vertical velocity components induced over a significant portion of the rotor disk. The exception being the edge of the rotor disk, which exist as numerical singularities in the vortex wake model. Despite the induced effects at the tips of physical rotors, caution must be taken when using this approach to model the inflow at the tips because the numerical singularities do not represents the actual physical behaviour of the shed tip vorticity.

\subsubsection{Representation of the Single Rotor Flow Field}

The flow field within the vicinity of a rotor can be shown by plotting the contours of the normal component of the induced velocity ratio. These are shown for various skew angles in Figs.5.28 through 5.33 and show agreeable comparison with the analytical solutions from Ref.53, that are included in Appendix C.1. As the skew angle increases, the inflow over the rotor disk is redistributed towards its aft section, similar to what is predicted with the first harmonic inflow models in Sec.3.3.6. Concentrated contours occur in the vicinity of the wake elements, which progressively become more spaced out as the tip speed ratio increases. The model neglects any blade-vortex interactions and assumes that the wakes are fixed and do not contract. The former assumption is evident in Fig.5.28 which show the tip vortices descending vertically from the rotor plane. 


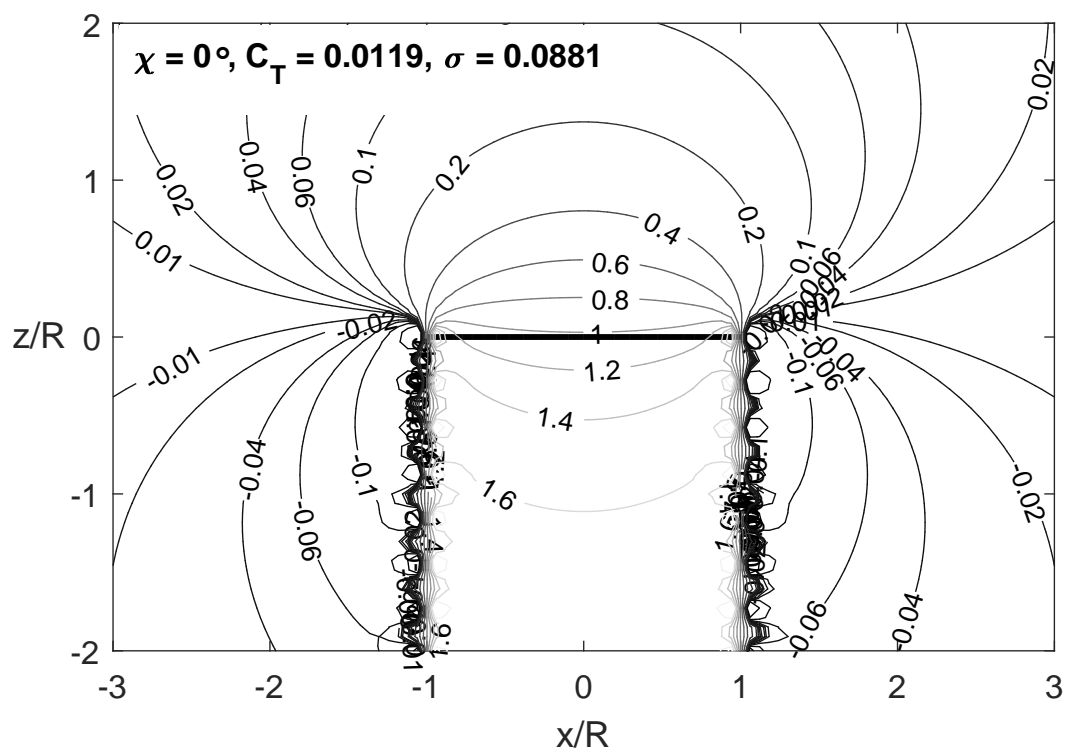

Figure 5.28: Lines of constant induced velocity for a single rotor flow field for $\chi=0^{\circ}$.

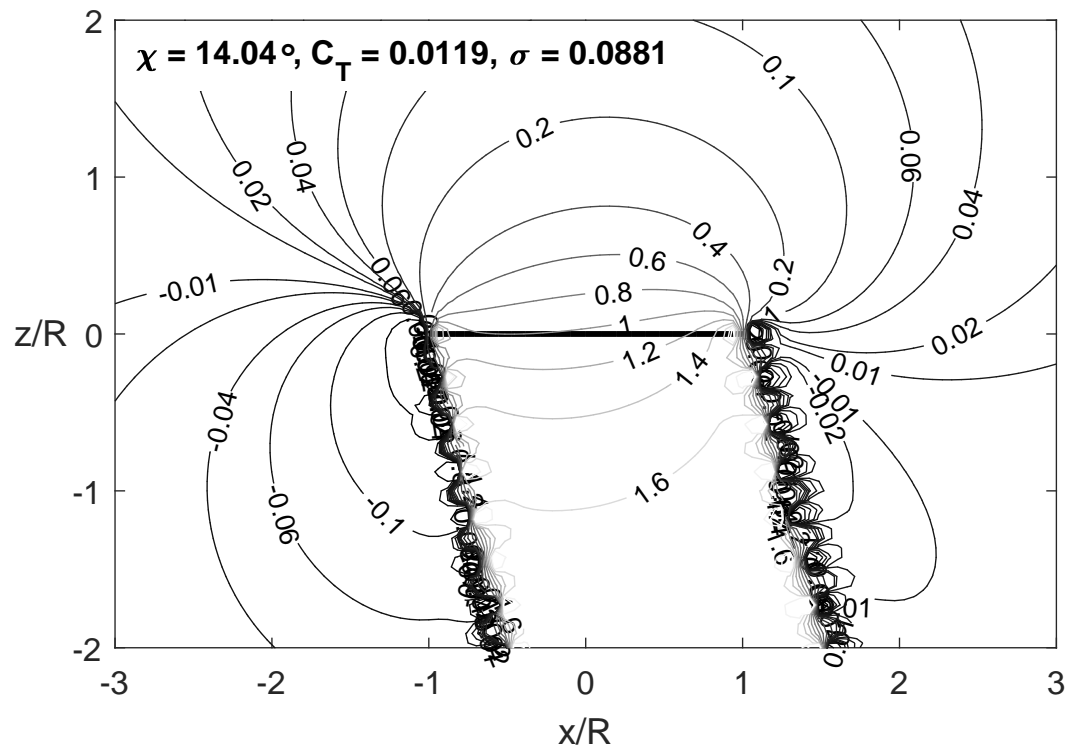

Figure 5.29: Lines of constant induced velocity for a single rotor flow field for $\chi=14.04^{\circ}$. 


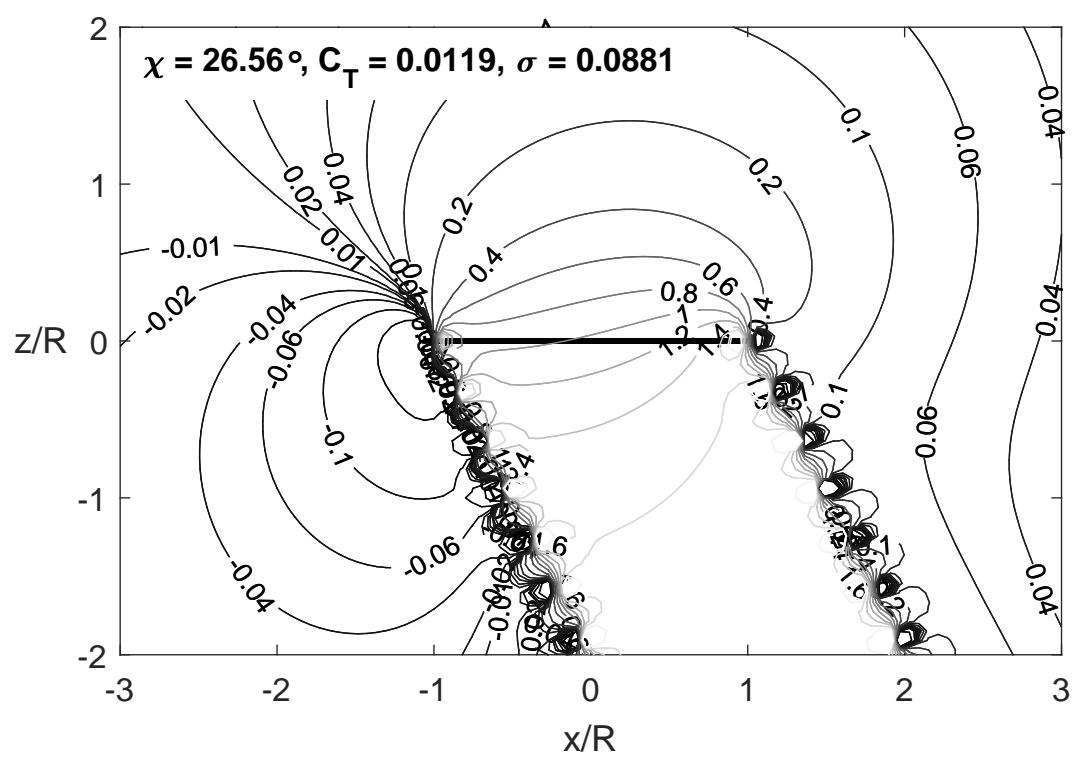

Figure 5.30: Lines of constant induced velocity for a single rotor flow field for $\chi=26.56^{\circ}$.

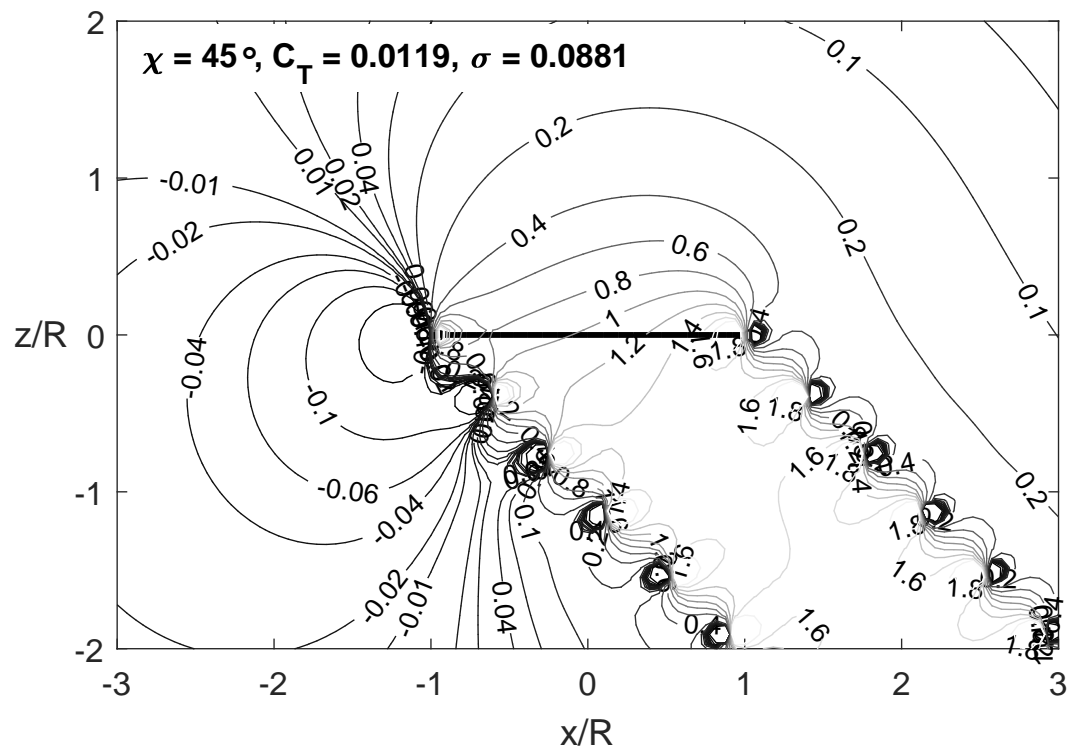

Figure 5.31: Lines of constant induced velocity for a single rotor flow field for $\chi=45^{\circ}$. 


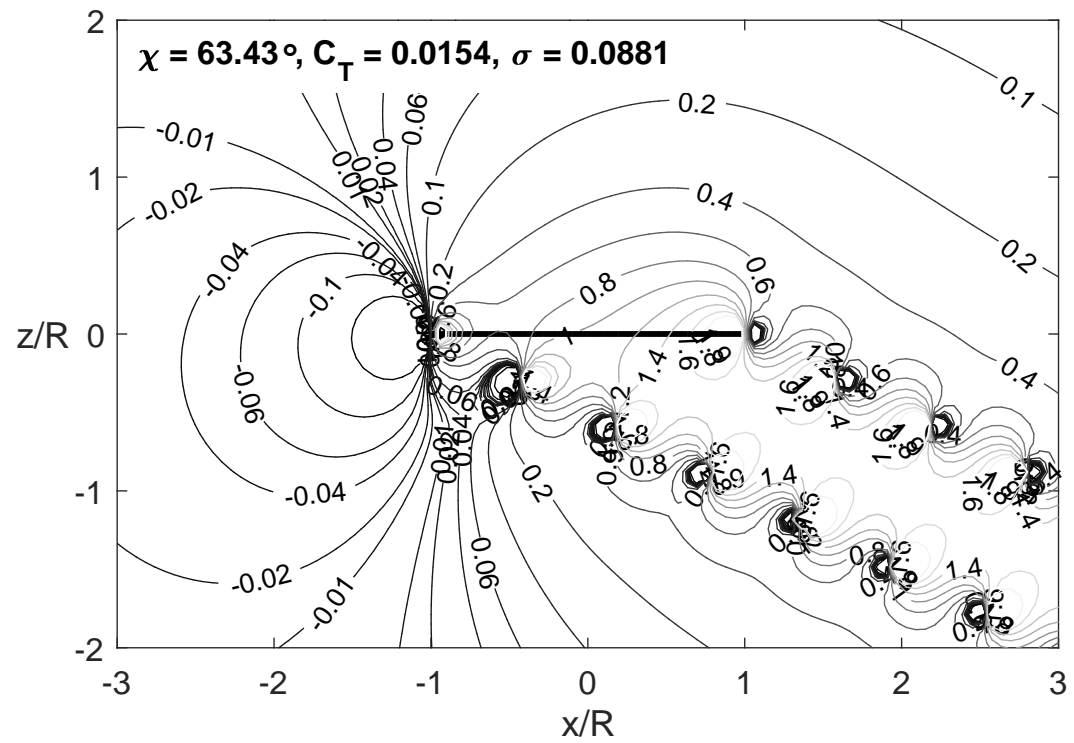

Figure 5.32: Lines of constant induced velocity for a single rotor flow field for $\chi=63.43^{\circ}$.

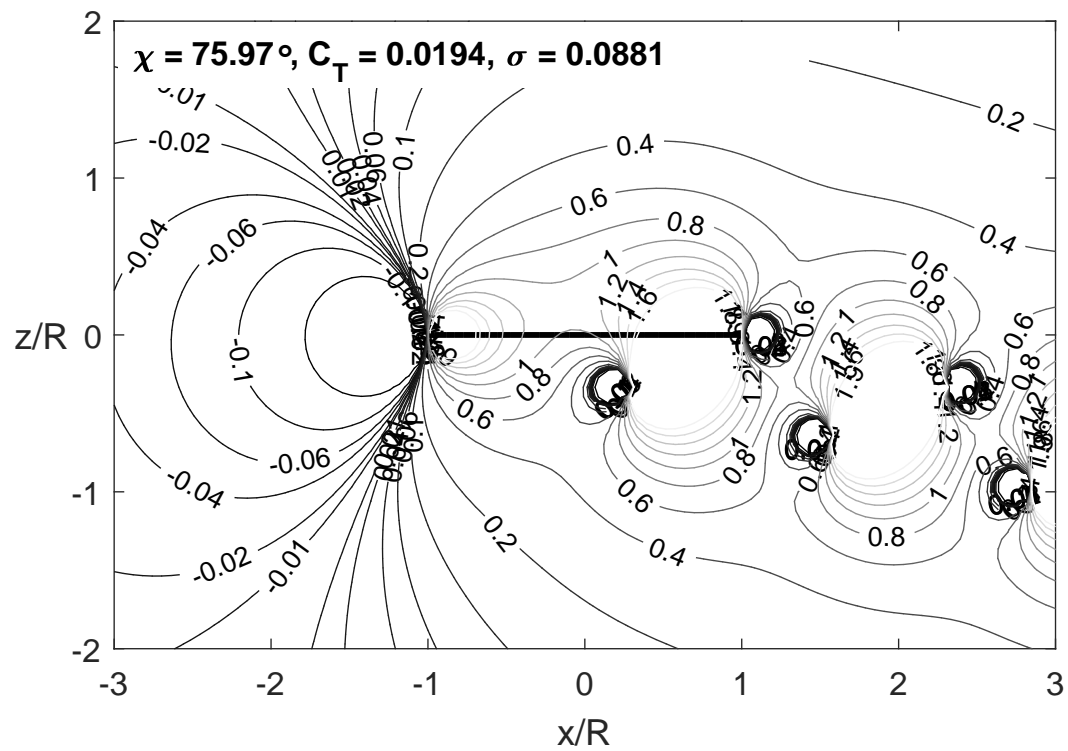

Figure 5.33: Lines of constant induced velocity for a single rotor flow field for $\chi=75.97^{\circ}$. 


\section{Chapter 6}

\section{Sample Applications}

This chapter consists of two sections that consist of applications for the models that were presented in Chapter 3. In the first section, a design methodology for rotors is presented that uses the single rotor performance model and a numerical optimization algorithm. The mathematical formulation of the rotor design problem is introduced that includes a discussion of the problem set-up, objective function, design variables and constraints. Two heuristic algorithms are considered, a genetic and a particle swarm method, and are evaluated with respect to their respective advantages for this type of problem. These were also used to also validate a design approach that uses a nested optimization algorithm instead of non-linear constraints. Following this, a number of example-design cases are presented for single and multi-point objectives in order to demonstrate the potential of this method as a design tool. In the second section, the wake induced velocities for a quad-configured SUAV are determined for several flight conditions, using the vortex wake model. The purpose of this is to investigate

whether these velocities contribute significantly to the rotor's inflow distributions in forward flight. 


\subsection{Rotor Design Using an Optimization Approach}

\subsubsection{Problem Formulation}

The effectiveness of a rotor design can be represented by an objective function, $f(X)$, which constitutes a metric that describes an aspect of its performance. For example, one can choose the power loading for a given thrust, when the design considers the aerodynamic efficiency of a single rotor. If $f(X)$ consists of variables, $X$, that represents characteristics of the rotor design, then the minimization problem is formulated as follows:

$$
\begin{gathered}
\min _{x \in \mathfrak{R}} f(X) \\
\text { subject to } A(X) \leq b \\
A_{e q}(X)=b \\
c(X) \leq 0 \\
c_{e q}(X)=0 \\
X_{L B} \leq X \leq X_{U B} \\
\text { where: } X=\left(x_{1}, x_{2}, \ldots, x_{n}\right) \\
X_{\mathrm{UB}}=\left[x_{\mathrm{UB}, 1}, x_{\mathrm{UB}, 2}, \cdots, x_{\mathrm{UB}, n}\right] \\
X_{\mathrm{LB}}=\left[x_{\mathrm{LB}, 1}, x_{\mathrm{LB}, 2}, \cdots, x_{\mathrm{LB}, n}\right]
\end{gathered}
$$

where $A(X)$ and $A_{e q}(X)$ are linear equality and inequality constraints, $c(X)$ and $c_{e q}(X)$ are non-linear equality and inequality constraints, and $X_{\mathrm{UB}}$ and $X_{\mathrm{LB}}$ are upper and lower bounds on the design variables.

The design parameters for the single rotor design case are grouped into three categories: the rotor geometry, trim and operating requirements, and the vehicle's flight dynamics. For the examples that are presented in this thesis, the vehicle's performance requirements, including its maximum weight and required thrust during hover and cruise, are given. The subsequent parameters which represent the vehicle's dynamic stability, are therefore kept constant. The 
remaining design variables and parameters are summarized in Table 6.1.

\begin{tabular}{|c|c|}
\hline Design Variables & Design Parameters \\
\hline \hline Radius & Vehicle operating conditions \\
\hline Number of blades & Atmospheric conditions \\
\hline Chord distribution & Required thrust \\
\hline Pitch distribution & Available power \\
\hline Sectional characteristics & Trim requirements \\
\hline Rotational speed & - \\
\hline
\end{tabular}

Table 6.1: Design variables and parameters for the generalized rotor design problem.

\subsubsection{Optimization Algorithm}

Most design optimization algorithms use either iterative or stochastic methods. Iterative solvers require a starting point and, for continuous objective functions, are proven to converge at the very least to a local minimum. Stochastic solvers, on the other hand, use heuristic searching methods, and hence, do not require an initial starting point. Although they are designed to find the global minimum, proof that they have converged on the global, and not on a local, minimum is not guaranteed. Despite this, stochastic solvers are capable of finding solutions to problems that have complex objective and constraint functions, such as those that are discontinuous or have numerous minima. Selecting an appropriate optimization algorithm depends on the scope of the problem, the starting criteria and the behaviour of the objective function.

Given the design variables in Table 6.1, which include integer design variables, the objective function is not guaranteed to be smooth and continuous. Furthermore, problems for which the objective function uses stochastic inputs are often referred to as "black box" problems, and often require the use of stochastic solvers. In particular, particle swarm and genetic algorithms, which are population based algorithms, are applicable to this type of problem and each have their own advantages $[64,65]$. The competing performance of both algorithms is discussed in Ref.66 which shows that generally particle swarm algorithms require fewer function evaluations 
compared to a genetic algorithm. However, the draw back in using a particle swarm approach is that its conventional implementation does not handle non-linear and integer constraints. Despite this, both approaches can be used for to problems that do not require non-linear constraints, and are both used herein in order to validate the optimization approach.

A disadvantage of stochastic methods is that they are less efficient in converging on a minimum compared to iterative methods, particularly steepest-descent methods, once they are in the region that contains the global minimum. Therefore, a hybrid optimizer is implemented in series and uses a candidate design from the heuristic method as its starting point. If the initial problem uses design variables or constraints that make the objective function discontinuous, there are two possible approaches to implementing the hybrid algorithm. One possibility is to fix the discontinuous variables from the heuristic search (i.e. number of blades and airfoil selection) so that a gradient based method can be used. Alternatively, a derivative-free method can be used that is able to handle the same variables and constraints such as the pattern search algorithm. In the present work, the second approach has been selected.

\subsubsection{Implementation of the Optimization Approach}

For most of the the rotor and propeller optimization approaches that are reviewed in Chapter 2 , the rotors tip speed is held constant and a non-linear equality constraint is used to ensure that the rotor produces a given thrust coefficient. For small fixed pitched rotors, however, this eliminates a large number of potentially feasible solutions that will be possible if the rotor is allowed to trim to a required thrust, irrespective of $C_{T}$. For this reason, an algorithm has been formulated, as shown in Fig.6.1, where the objective function also includes a nested-optimization scheme for the rotor's trim. The trim method consists of a single variable bounded minimization problem using a non-linear least-squares algorithm in order to determine the required rotational speed to produce a required thrust. A by-product of adding the trim subroutine is that all non-linear constraints are eliminated. 


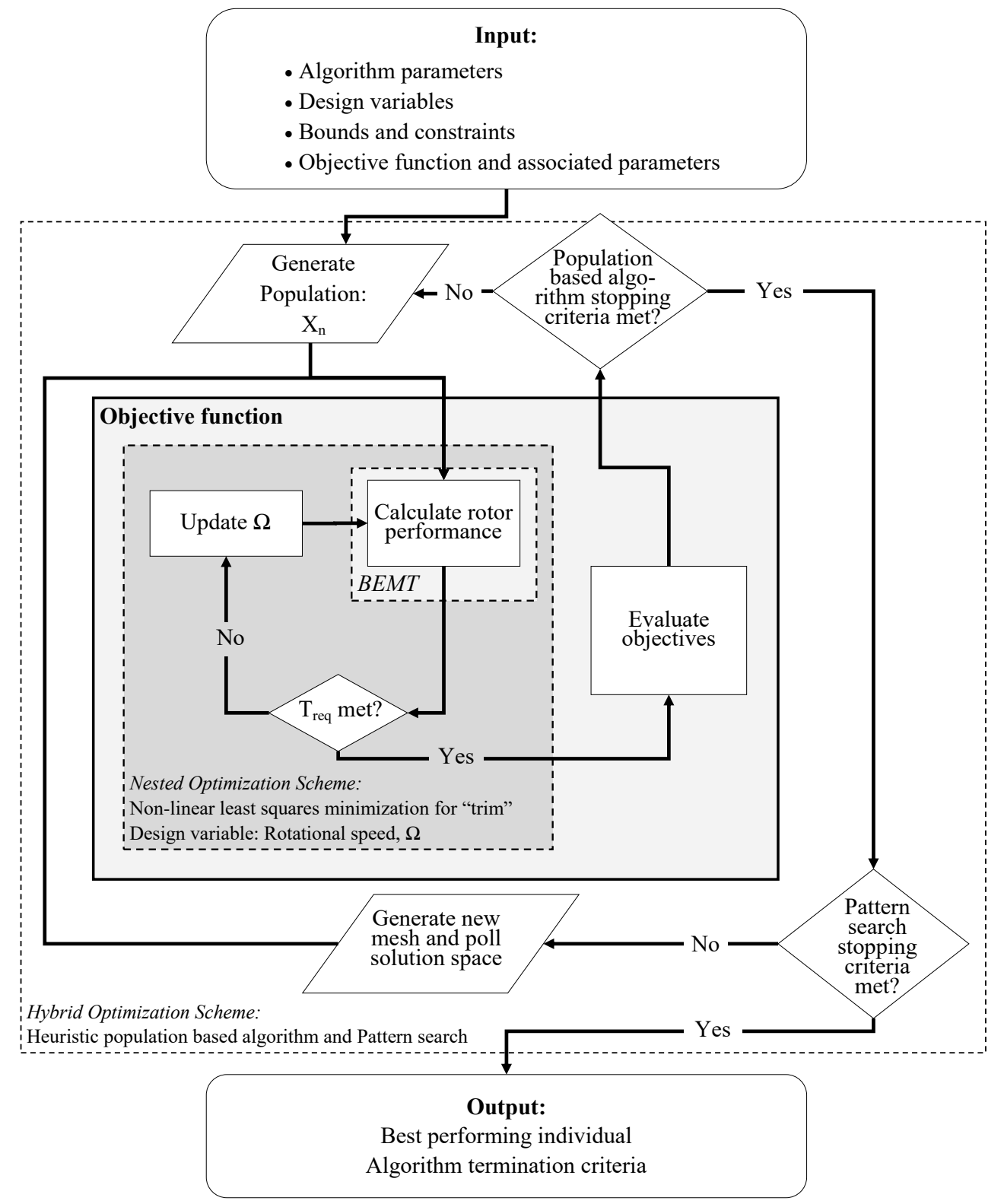

Figure 6.1: Optimization approach flow chart.

\subsubsection{Blade Parametrization}

The radial chord and twist distributions are represented using control points that define the shape of a piecewise cubic hermite interpolating polynomial (pchip spline), as is shown in Fig.6.2. The advantage of using this approach is that the first derivatives of the chord and pitch 
distributions, $\frac{d \frac{c}{R}}{d r}$ and $\frac{d \beta}{d r}$, are continuous. First-derivative continuity implies that the generated blade design is smooth and therefore manufacturable. If more strict manufacturing constraints are required, a cubic spline can be used instead, with an inequality constraint on its second derivative. Another advantage of using pchip splines is that they result in no overshoot, and so the bounds that are applied to the control points also apply to the twist and chord distributions within the regions neighbouring the control points. In order to translate these control points into radial chord and twist distributions, the phcip spline is evaluated for 25 span-wise stations with the following radial distributions:

$$
r_{n+1}-r_{n}= \begin{cases}0.05, & r<0.8 \\ 0.02, & r \geq 0.8\end{cases}
$$

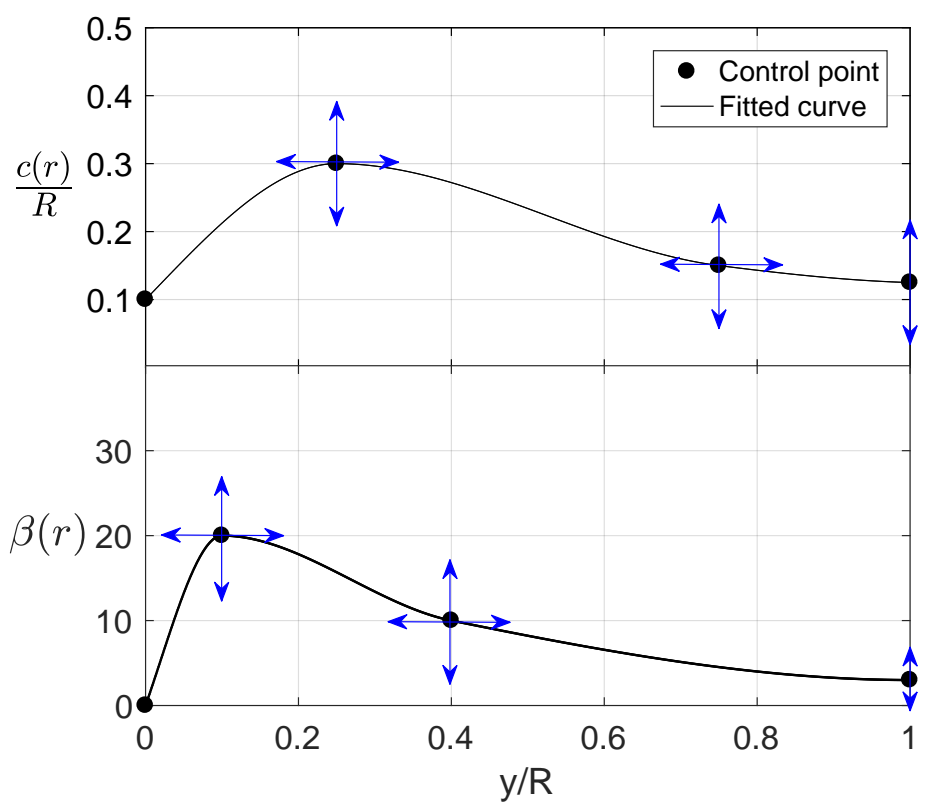

Figure 6.2: Blade chord and twist distributions. 


\subsection{Validation of the Approach}

\subsubsection{The Ideally Twisted Blade for Hover}

In order to validate the design methodology that is described in the previous section, the twist distribution required for minimum induced power in hover was computed for a inviscid, constant-thickness rectangular rotor operating at a constant thrust loading. As was discussed in Chapter 2, this coincides with the analytical findings that the optimized blade has a uniform inflow distribution, which requires a hyperbolic radial twist distribution when using small angle approximations and neglecting tip-losses. The results were obtained using the nested optimization approach of Fig.6.1 in addition to an approach that assumes a constant tip speed and, therefore, uses a non-linear constraint on the required thrust. Both cases use the genetic algorithm and are compared to the analytical result. The design parameters used are summarized in Table 6.2.

\begin{tabular}{|c|c|c|}
\hline Required thrust & 5.375 & {$[\mathrm{~N}]$} \\
\hline Radius & 0.22 & {$[\mathrm{~m}]$} \\
\hline Blades & 2 & - \\
\hline Solidity & 0.0868 & - \\
\hline Airfoil & NACA 0012 & - \\
\hline Rotational speed & $3000-8000$ & {$[\mathrm{RPM}]$} \\
\hline
\end{tabular}

Table 6.2: Design variables for the validation of the optimization approach.

Figures 6.3 and 6.4 below show the twist distributions obtained for the nested optimization and constrained optimization schemes, respectively. The objective function for both cases was to minimize the induced power required in hover. The nested scheme was run first, and then the rotational speed that was selected for this design was used as the rotational speed for the constant tip-speed approach. 


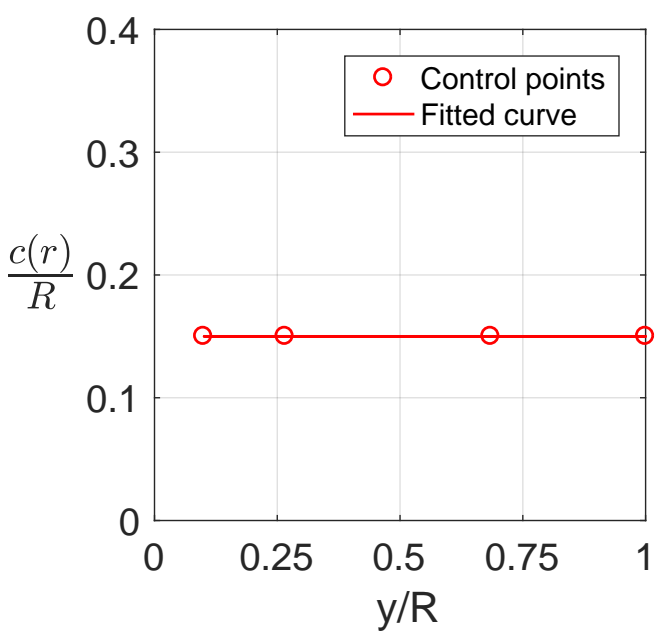

(a) Chord distribution

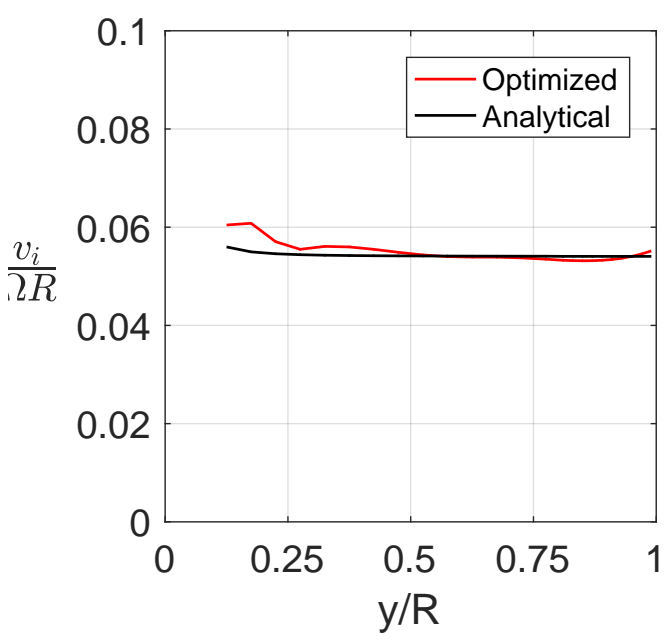

(c) Inflow distribution

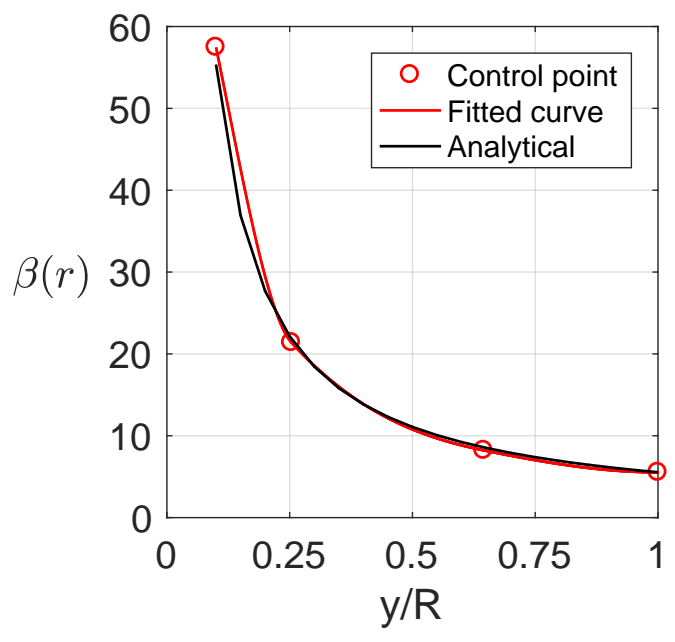

(b) Twist distribution (degrees)

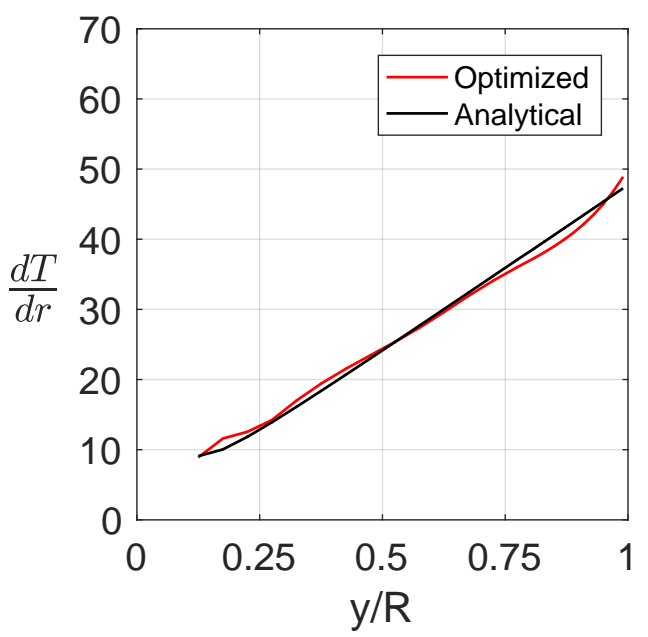

(d) Thrust distribution

Figure 6.3: Comparisons between the optimized and analytical twist distributions for a hovering rotor using the nested trim method 


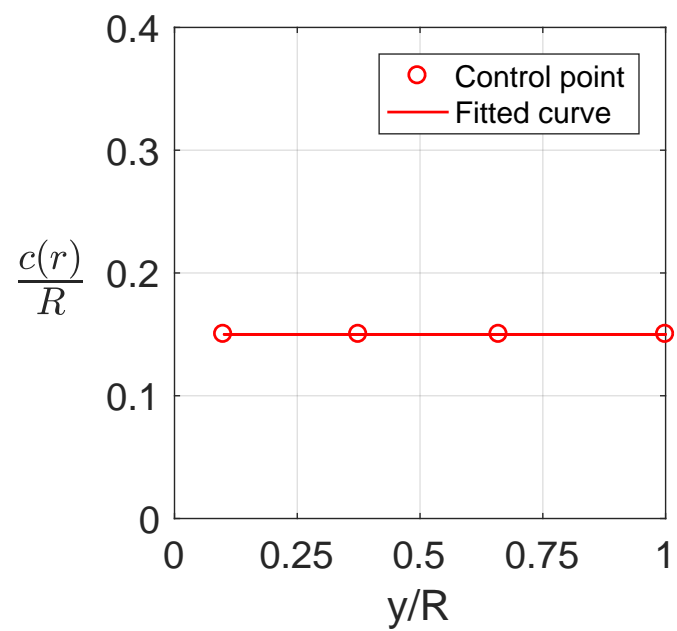

(a) Chord distribution

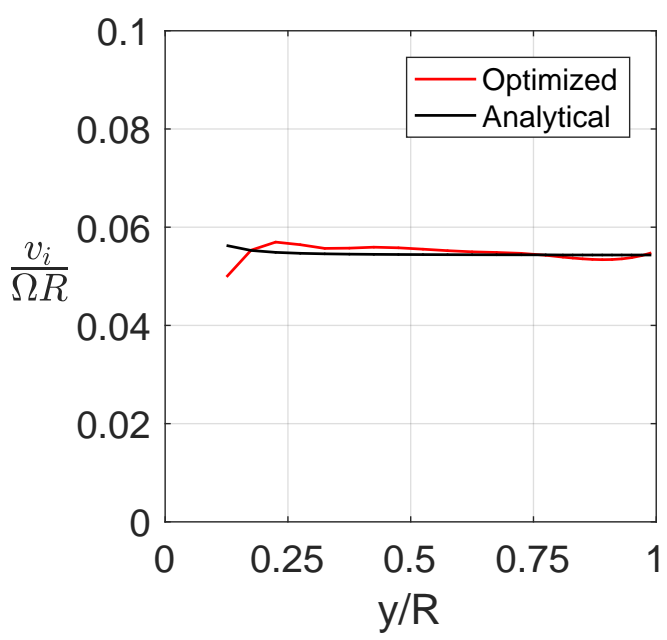

(c) Inflow distribution

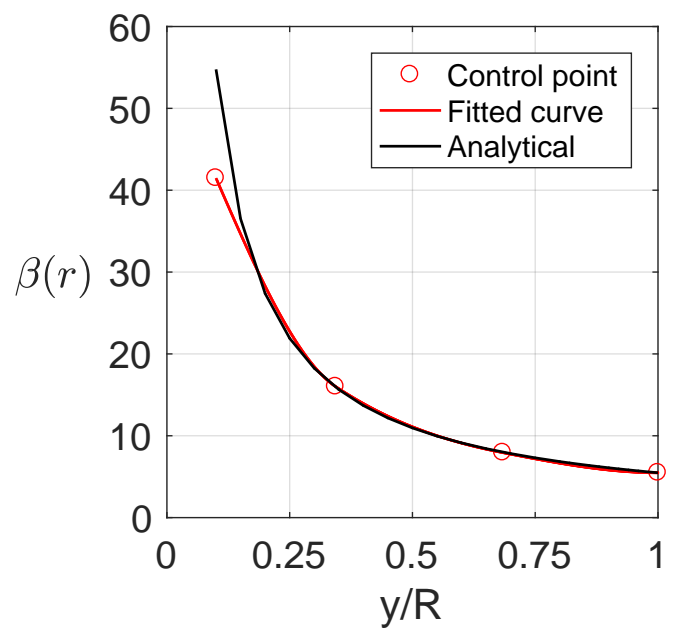

(b) Twist distribution (degrees)

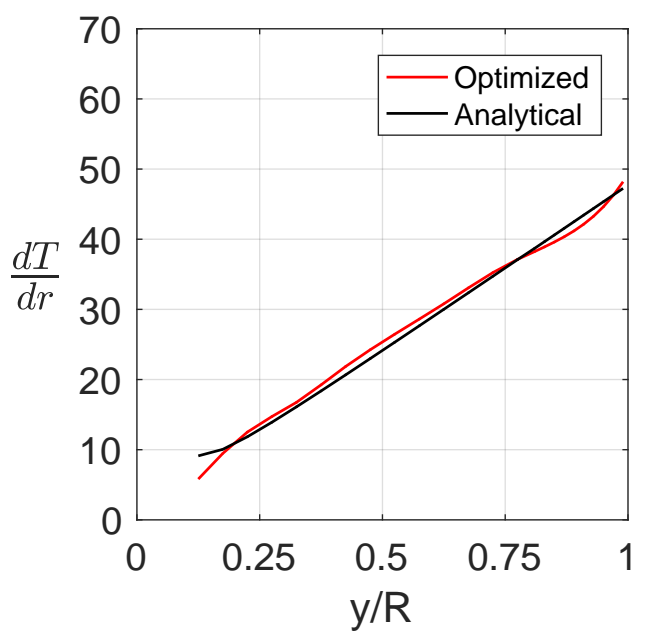

(d) Thrust distribution

Figure 6.4: Comparisons between the optimized and analytical twist distributions for a hovering rotor using the constrained tip-speed method

In both instances, the radial twist distributions closely match the ideal twist distributions of the analytical results and both produce relatively uniform downwash. Minor differences exist close to the hub as a result of these sections having a small contribution to the cumulative induced power and partially because the small angle approximations assume that the section lift is equal to the thrust. Furthermore, based on the problem's stopping criteria, minor changes to 
the twist distribution at the root have a negligible effect on improving the objective function and, therefore, the algorithm determines that the problem has converged.

For the above two cases, the genetic algorithm was implemented using identical solver characteristics, notwithstanding the non-linear thrust constraint, to verify the repeatability of the results. In order to validate that these results are also repeatable when using a different solver, the nested optimization scheme was implemented using a particle swarm algorithm. Figure 6.5 compares the radial twist distributions that were obtained using the particle swarm algorithm, in addition to the results from the genetic algorithm. Table 6.3 compares the induced power coefficients obtained using each approach. For the three cases, the particle swarm algorithm required slightly less computational time.

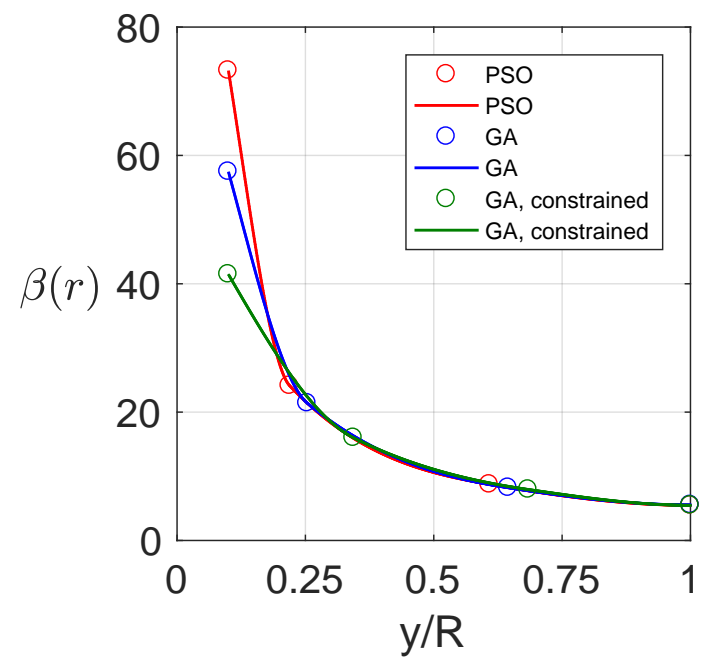

Figure 6.5: Optimized twist distributions generated by GA and PSO algorithms

\begin{tabular}{|c|c|c|c|}
\hline Algorithm & $\mathbf{C}_{\mathbf{T}}$ & $\mathbf{C}_{\mathbf{P i}}$ & $\boldsymbol{\Omega}[\mathrm{RPM}]$ \\
\hline \hline Genetic Algorithm & 0.006 & 0.0003267 & 3000 \\
\hline Constrained Genetic Algorithm & 0.006 & 0.0003277 & 3000 \\
\hline Particle Swarm & 0.006 & 0.0003264 & 3000 \\
\hline
\end{tabular}

Table 6.3: Comparison of results obtained using several optimization approaches. 


\subsection{Example Designs}

In this section, three design cases are presented using single and multipoint design objectives. A secondary purpose of these cases is also to investigate the performance of rotors having multiple blades. The operating conditions considered are representative of a small quadconfigured sUAV having a typical operating weight of 3.75 kilograms, with payload, and a cruise speed of 35 knots. A vehicle of this type is designed to have maximum endurance for missions that require a small payload, for example, a small video camera for aerial surveillance. The maximum rotor radius is $0.228 \mathrm{~m}$, which is limited by the airframe configuration given by the length of the rotor-support arms. The maximum continuous power available for each rotor is $275 \mathrm{~W}$. A commercially available rotor with the same radius is used as a baseline to compare the performance of the designs generated using the optimization methodology. The baseline rotor operates at a rotational speed of approximately $445 \mathrm{rad} / \mathrm{s}$ at full throttle. In hover, the rotor performance model predicts that the power requirement for the baseline design is $45 \mathrm{~W}$ and coincides with a rotational speed of $254 \mathrm{rad} / \mathrm{s}$ for a $40-50 \%$ throttle setting. The optimized rotor designs are assumed to have the same airfoil and thickness distribution as the baseline geometry. For these cases, the genetic algorithm is selected over the particle swarm algorithm due to the inclusion of integer constraints that represent the number of blades.

\subsubsection{Hover}

For the first design, the objective function is the minimum required power during hover. Table 6.4 summarizes the bounding constraints that were placed on the design variables. Four control points were used to represent twenty-five blade stations that were placed along the blade radius. Two designs were generated with two and three blades, respectively. For the threebladed design, the bound constraint on the minimum number of blades was increased to 3 . The performance of both geometries are summarized in Table 6.5. 


\begin{tabular}{|c|c|c|c|}
\hline Design Variable & Upper Bound & Lower Bound & Units \\
\hline \hline Radius, $R$ & 0.228 & 0.128 & {$[\mathrm{~m}]$} \\
\hline Blades, $N_{b}$ & 2 & 5 & - \\
\hline Control point position & {$[0.1,0.30,0.80,1]$} & {$[0.1,0.20,0.35,1]$} & - \\
\hline Section pitch, $\beta(r)$ & {$[2,25,15,15]$} & {$[2,10,5,5]$} & {$[\mathrm{deg}]$} \\
\hline Section chord, $c(r) / R$ & {$[0.08,0.25,0.20,0.15]$} & {$[0.08,0.10,0.10,0.05]$} & - \\
\hline Rotational speed, $\Omega$ & 445 & 209.4 & {$[\mathrm{rad} / \mathrm{s}]$} \\
\hline
\end{tabular}

Table 6.4: Bound constraints on the design variables.

Compared to the baseline rotor, the two-bladed rotor performs similarly, whereas the threebladed rotor requires $10 \%$ more power. For both designs, the primary driver in minimizing the required power comes from reducing the rotational speed and increasing the rotor solidity. For instance, in Fig.6.6, the larger solidity is achieved by increasing the blade planform through collectively increasing the radial chord distribution. For the second design, the solidity is increased by using another blade. For both designs, the blades are slightly more twisted than the baseline and have an increased inflow distribution along the blade. Despite this, the optimized designs also produce inflow distributions which resemble uniform downwash for a portion of the outer blade. Comparing the two- and three-bladed designs demonstrates the trade-off between the power savings obtained by lowering the rotor's rotational speed versus having blade planforms that minimize the profile and induced drag. Using the three-bladed rotor as an example, once the intermediary candidate designs that are generated by the algorithm more or less coalesce at the minimum allowable rotational speed, they receive no additional benefit from increasing their solidity. The algorithm therefore explores designs that instead minimize the profile losses, while generating designs that maintain the lowest rotational speed. Thus, a chord distribution similar to the idealized chord distribution for minimizing profile and induced losses in Ref.29 is obtained. For the two-bladed case, the larger planform is needed to generate the required thrust for a minimum rotational speed, and so in order to obtain this condition, the chord length was maximized at two of the control point positions. 


\begin{tabular}{|c|c|c|c|c|}
\hline Design & Power loading, $\frac{T}{P}[\mathbf{N} / W]$ & $\Omega[\mathbf{r a d} / \mathbf{s}]$ & Radius [m] & Blades \\
\hline \hline Baseline & 0.2149 & $254.4(2429.3 \mathrm{RPM})$ & 0.228 & 2 \\
\hline Design 1 & 0.2184 & $209.5(2000 \mathrm{RPM})$ & 0.228 & 2 \\
\hline Design 2 & 0.1935 & $209.5(2000 \mathrm{RPM})$ & 0.228 & 3 \\
\hline
\end{tabular}

Table 6.5: Characteristics of two- and three-bladed rotor designs in hover.

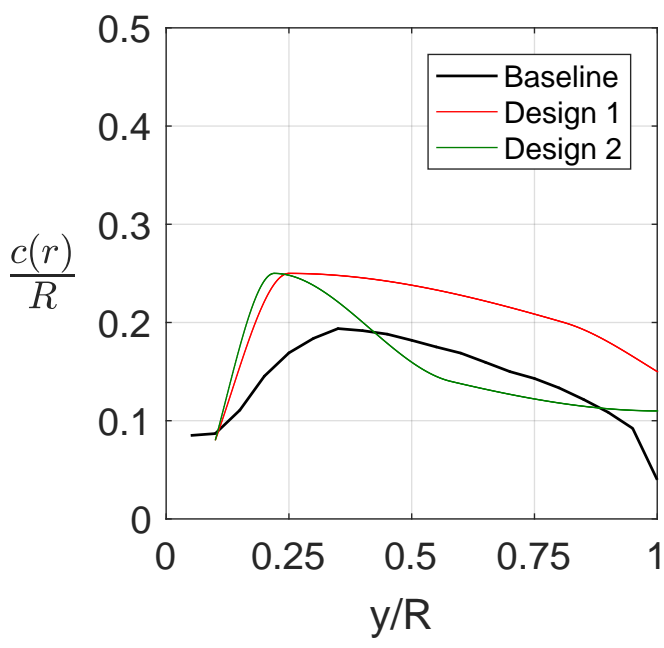

(a) Chord distribution

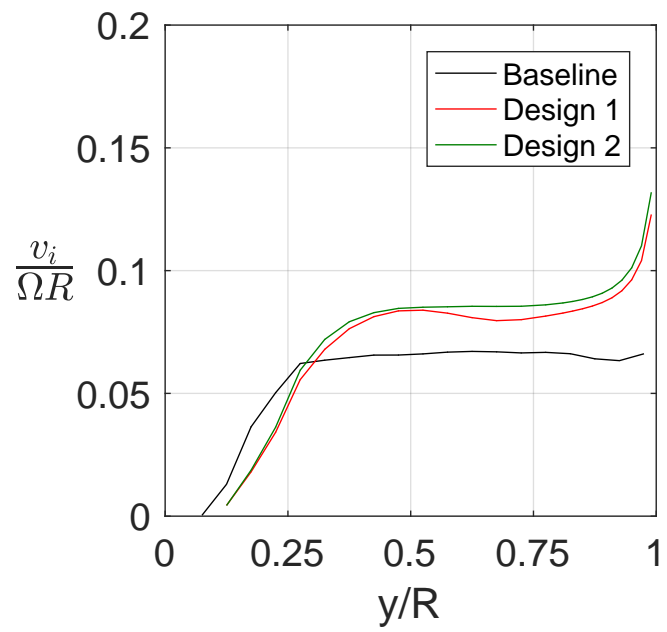

(c) Inflow distribution

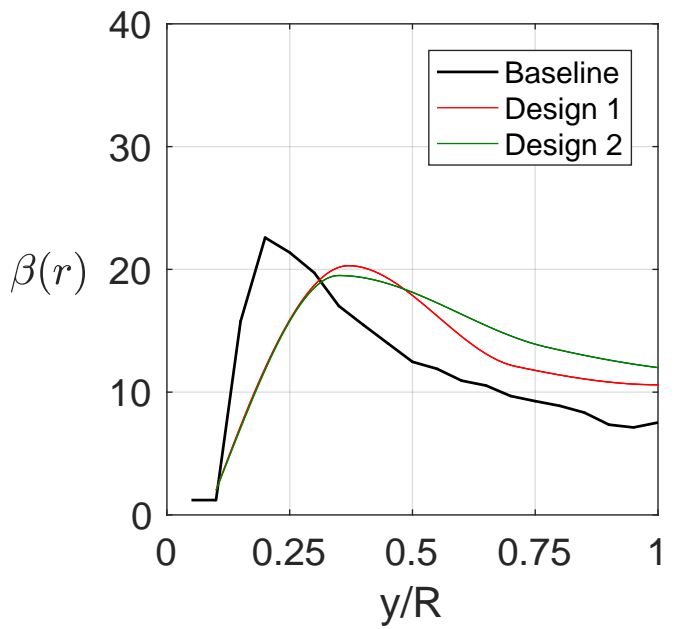

(b) Twist distribution (deg)

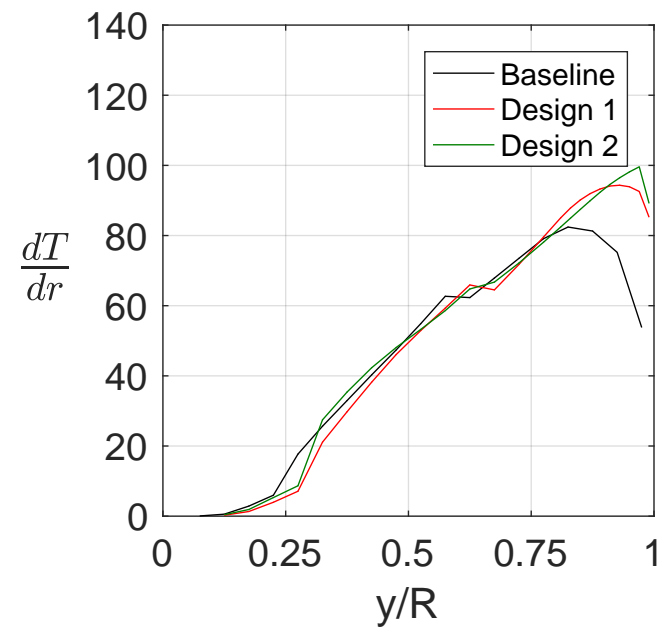

(d) Thrust distribution

Figure 6.6: Comparisons between the baseline and optimized designs for a hovering rotor. 


\subsubsection{Edgewise Flight}

To determine a rotor geometry that is designed specifically for edgewise flight, a single performance point optimization was performed for the cruise condition. The computational expense for the edgewise flight condition is larger than for the hovering case due to the solution requiring simultaneous iteration routines for both the rotor's thrust and inflow distribution. For this reason, only a single design was completed to demonstrate the potential of the design methodology for this flight regime.

A representation of the forces acting through the rotor hub is shown in Fig. 6.7. This case assumes that the cumulative effect from the pitching moments and side forces of all the vehicle's rotors and UAV's airframe are in equilibrium. In particular, the forces are thrust, $T$, the in-plane rotor force, $F_{x}$, body drag, $D$, and weight, $W$. Also included in the graph is the lift force produced by the fuselage.

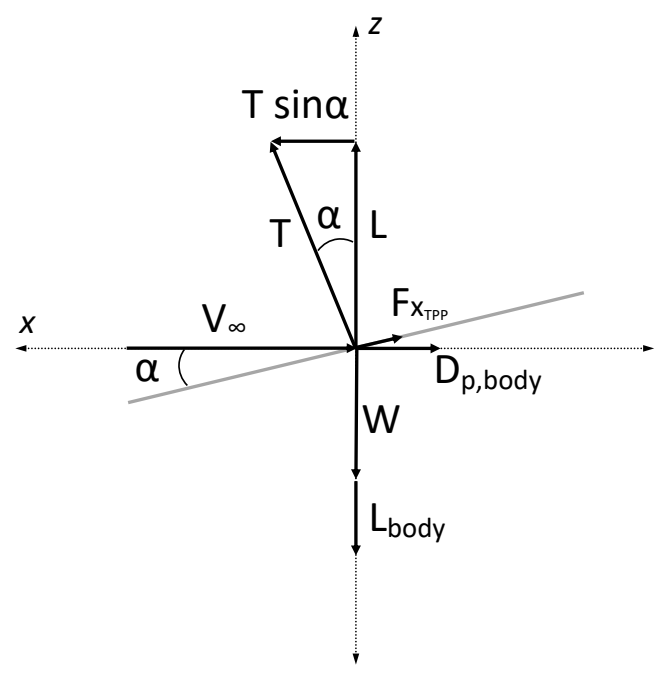

Figure 6.7: Rotor trim in forward flight.

The forces about the hub can be resolved into lift and drag components: 


$$
\begin{array}{r}
T \sin \alpha-F_{x_{\mathrm{TPP}}} \cos \alpha-D_{\mathrm{p}, \text { body }}=0 \\
T \cos \alpha-F_{x_{\mathrm{TPP}}} \sin \alpha-W-L_{\text {body }}=0
\end{array}
$$

The forces resulting from the vehicle cruising at $10 \mathrm{~m} / \mathrm{s}$ are summarized in Table 6.6 below. The limits on the design variables are similar to the ones listed in Table 6.4 for the hover case with the exception being that the allowable sectional chord for the first control point is increased to 0.3. The objective function is the minimum the power required for the required thrust in cruise.

\begin{tabular}{|c|c|c|}
\hline Thrust, T & 10.1 & {$[\mathrm{~N}]$} \\
\hline$D_{\text {p,body }}$ & 2.65 & {$[\mathrm{~N}]$} \\
\hline$L_{\text {body }}$ & 2.25 & {$[\mathrm{~N}]$} \\
\hline Angle of attack, $\alpha$ & 15 & Deg. \\
\hline
\end{tabular}

Table 6.6: Additional vehicle forces in edgewise cruising flight.

The operating conditions and geometry of the optimized blade is summarized in Table 6.7 and Figs.6.8, respectively. The optimized blade geometry shows a power savings of approximately $15 \%$, which similar to the hover case, is accomplished by lowering the tip speed and increasing the solidity. Both the twist and chord distributions for the edgewise flight case, shown in Fig.6.8, are similar to the idealized hyperbolic distributions, although less twist is used compared to the hover case. In addition to the optimized blade's larger solidity, the radial chord distribution within the tip region follows a different trend, shown in Fig.6.8a, for which the baseline rotor has rounded tips. An explanation for the difference in tip geometry, notwithstanding the reasons related to manufacturing or damage tolerance, is as a result of the tip effects not being modelled in detail using the linear inflow approximations. Although implementing tip designs that use sweep, variable planform and anhedral can impact rotor performance [13], capturing the full tip effects during edgewise flight and generating designs that consider the performance of optimized blade tips, requires the use of a more comprehensive models. 
Figures 6.9 through 6.11 show the distributions of the sectional thrust, power and induced power. Compared to the baseline design, the optimized rotor generates more thrust on the inboard advancing blade in addition to more being distributed inboard. The induced drag, which is the largest contributor to the total power, is also reduced on the retreating side as a result of the reduced thrust. Although the optimized rotor design requires less power for this particular flight condition, there are two drawbacks that, insinuated from Figs 6.9 through 6.11, become an issue at higher forward flight speeds. First, at higher advance ratios a large portion of the retreating blade operates near or well within the stall region. Second, an increased rolling moment arises from the uneven lateral thrust distribution. Subsequently, trimming the vehicle can become an issue. Although not done for the herein presented results, these effects can be included as design parameters, for example by limiting the amount of blade radius being stalled.

\begin{tabular}{|c|c|c|c|c|}
\hline Case & Power loading, $\frac{T}{P}[\mathbf{N} / \mathbf{W}]$ & $\Omega[\mathbf{r a d} / \mathbf{s}]$ & Radius $[\mathbf{m}]$ & Blades \\
\hline \hline Baseline & 0.1868 & $286.2(2733 \mathrm{RPM})$ & 0.228 & 2 \\
\hline Optimized & 0.2170 & $211.5(2019.7 \mathrm{RPM})$ & 0.228 & 3 \\
\hline
\end{tabular}

Table 6.7: Characteristics of a three-bladed design for edgewise flight.

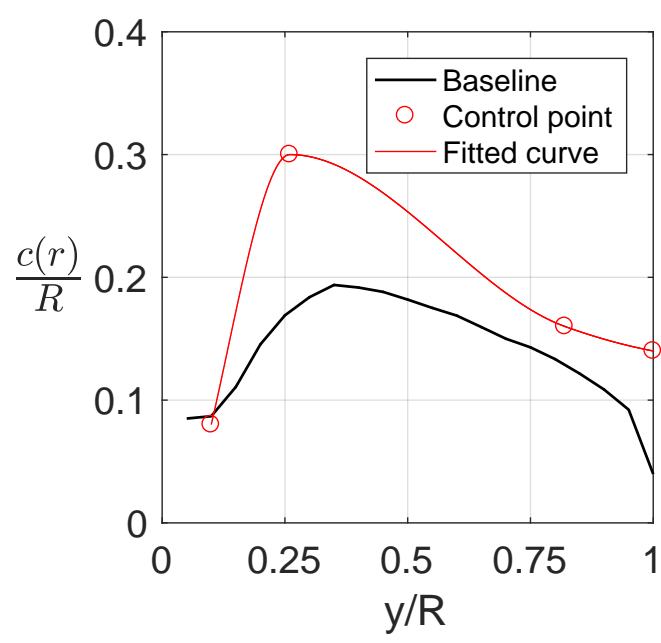

(a) Normalized chord distribution.

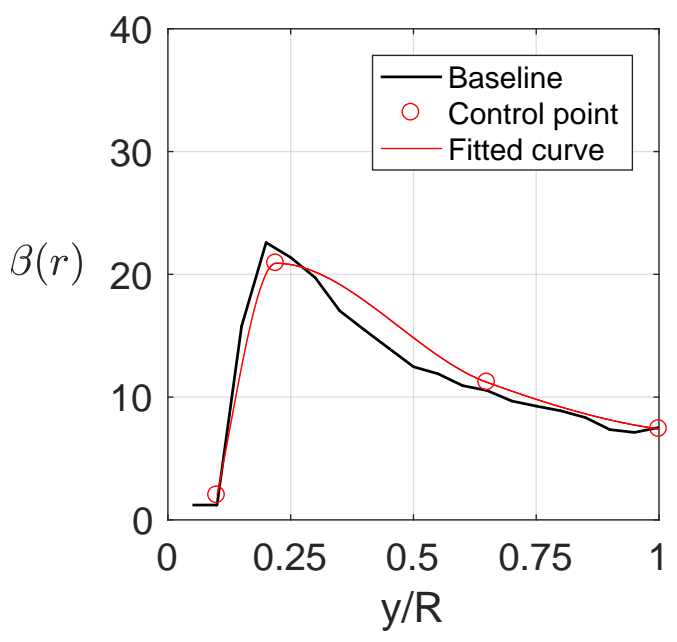

(b) Twist distribution (deg).

Figure 6.8: Comparisons between the baseline and optimized geometries for a rotor designed for edgewise flight. 


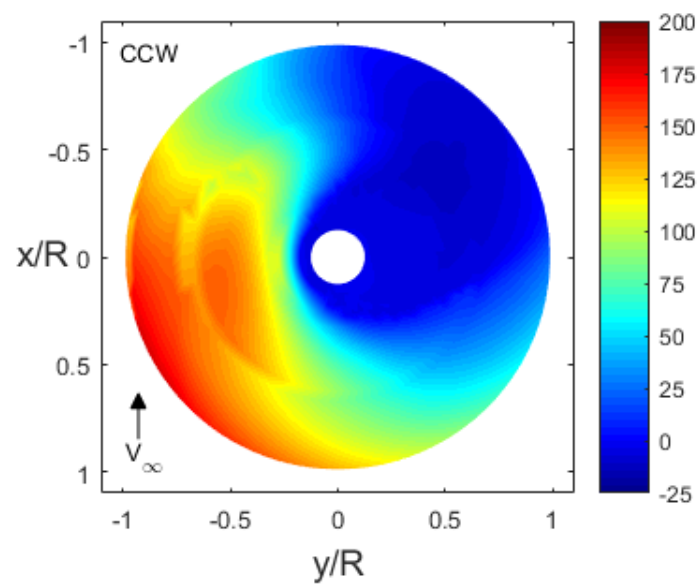

(a) Optimized.

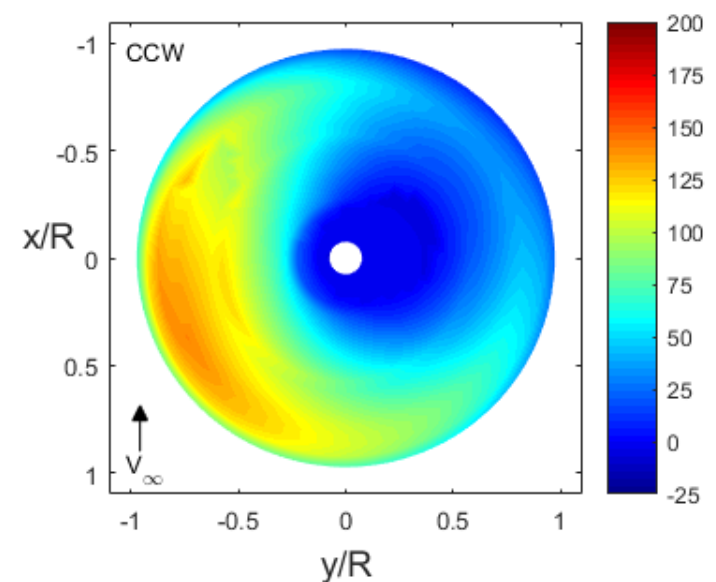

(b) Baseline.

Figure 6.9: Section thrust per unit span, $\frac{d T}{d r}\left[\frac{N}{m}\right]$ (rotor spins counter-clockwise).

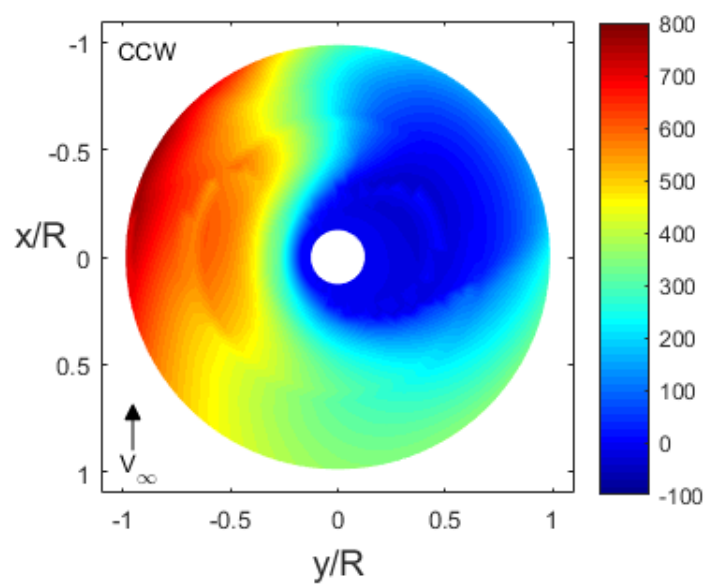

(a) Optimized.

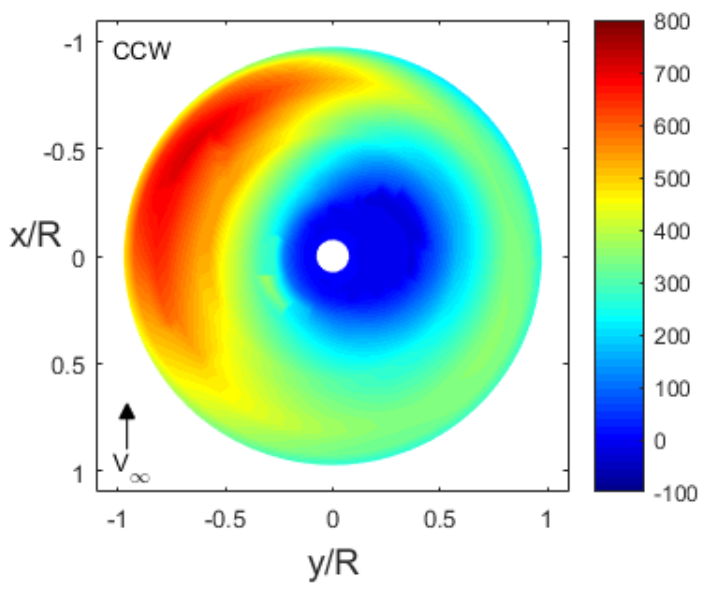

(b) Baseline.

Figure 6.10: Section power per unit span, $\frac{d P}{d r}\left[\frac{W}{m}\right]$ (rotor spins counter-clockwise). 


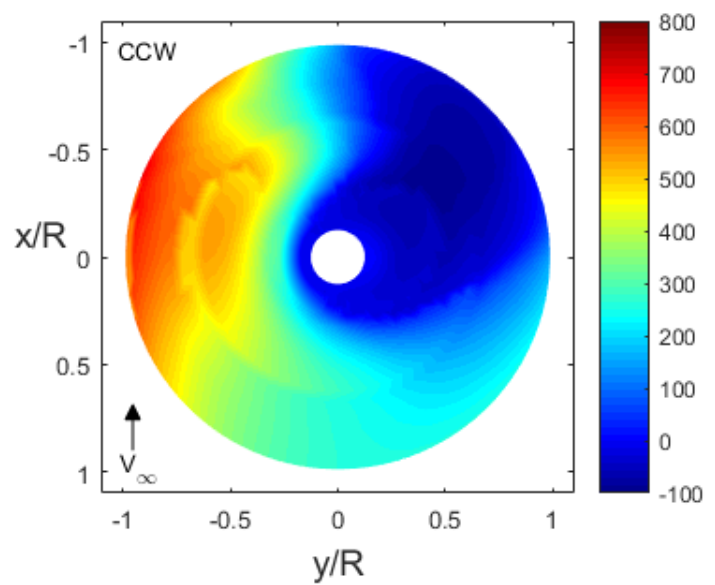

(a) Optimized.

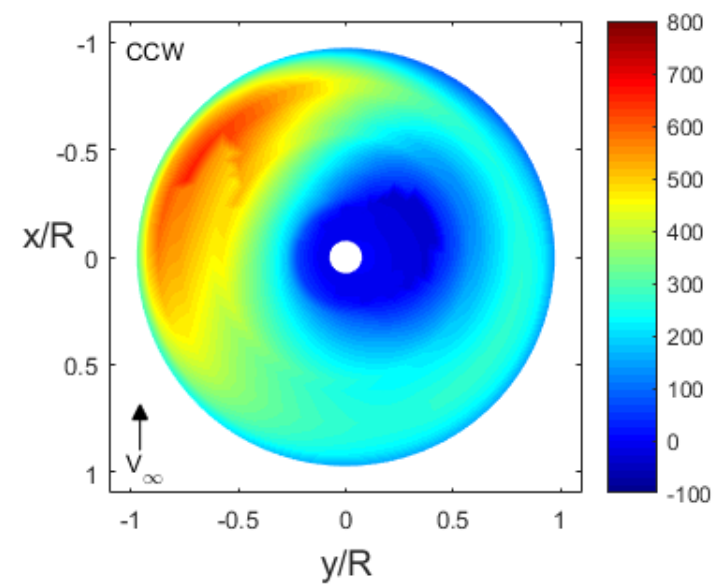

(b) Baseline.

Figure 6.11: Section induced power per unit span, $\frac{d P_{i}}{d r}\left[\frac{W}{m}\right]$ (rotor spins counter-clockwise).

\subsubsection{Multi-objective Hover and Edgewise Flight}

The designs that were generated for the hover and edgewise flight condition differ in their respective solidity and twist distributions. Thus, several designs that combine these conditions are presented using an objective function that consists of the weighting of the required power in hover and in edgewise flight. The design variables and operating parameters from the previous two cases are used and the vehicle is assumed to spend an equal time in hover and cruise/loiter. The objective function is the combined thrust loading between each flight condition and is given by:

$$
f(x)=\frac{T_{\text {Hover }}+T_{\text {Edgewise }}}{P_{\text {Hover }}+P_{\text {Edgewise }}}
$$

As listed in Table 6.8, compared to the baseline, the three- and two-bladed designs show improvements of $4.9 \%$ and $13.9 \%$ respectively. The net increase in solidity for both designs is similar, where the first case accomplishes this by adding an additional blade and the second has a larger planform. Both twist distributions follow a trend similar to that of the baseline 
geometry, which again, resembles the hyperbolic twist distribution. Both cases yield similar inflow distributions to the baseline blade for the hover case, shown in Fig.6.13, which is uniform for the outer portion of the blade.

\begin{tabular}{|c|c|c|c|c|c|}
\hline Case & $\frac{T}{P}[\mathbf{N} / \mathbf{W}]$ & $\Omega_{\mathbf{H}}[\mathbf{r a d} / \mathbf{s}]$ & $\Omega_{\mathbf{E}}[\mathbf{r a d} / \mathbf{s}]$ & Radius $[\mathbf{m}]$ & Blades \\
\hline \hline Baseline & 0.20 & $254.4(2429.3 \mathrm{RPM})$ & $286.2(2733 \mathrm{RPM})$ & 0.228 & 2 \\
\hline Design 1 & 0.21 & $215.6(2058.8 \mathrm{RPM})$ & $241.3(2304.3 \mathrm{RPM})$ & 0.228 & 3 \\
\hline Design 2 & 0.22 & $213.7(2040.7 \mathrm{RPM})$ & $246.7(2355.8 \mathrm{RPM})$ & 0.228 & 2 \\
\hline
\end{tabular}

Table 6.8: Characteristics of two- and three-bladed designs for hover and edgewise flight.

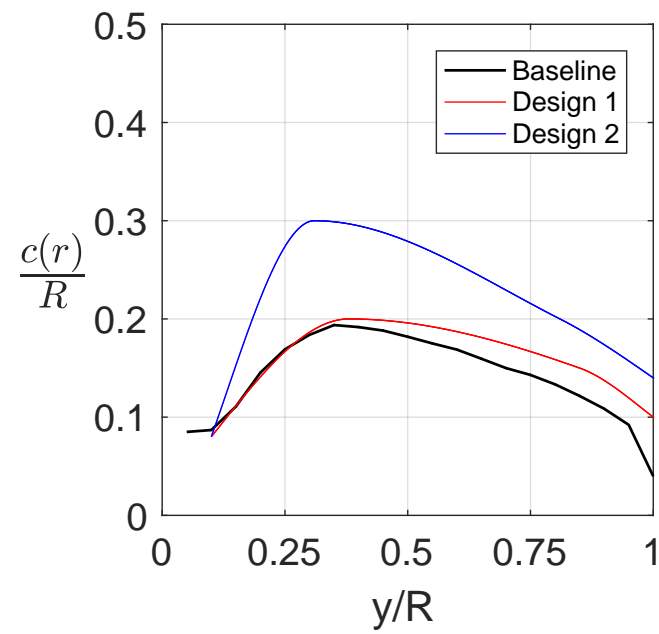

(a) Normalized chord distribution

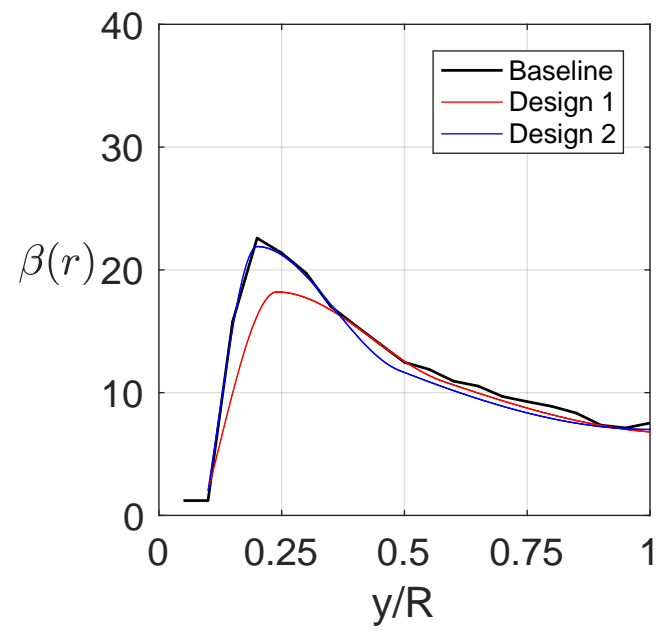

(b) Twist distribution (deg)

Figure 6.12: Comparisons between the baseline and optimized rotor geometries for the multipoint design case. 


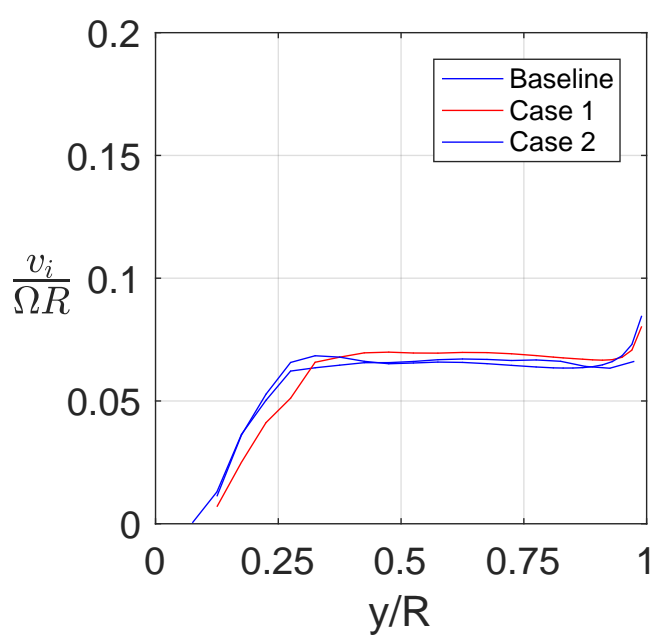

Figure 6.13: Comparisons of the inflow distributions for the multi-point designs in hover.

The power savings are achieved primarily due the reduction of the induced power on the retreating blade, as shown in Figs.6.15 and 6.16 for the three- and two-bladed designs, and for the baseline in Figs.6.10 and 6.11. Comparing the two-bladed and three-bladed designs, a reduction in induced power is achieved by the two-blade design by having a more smoothly distributed thrust loading on the advancing side. Although both rotors operate at similar rotational speeds, the two-bladed design requires approximately $9 \%$ less power. In addition to the induced losses, a small portion of the power reduction is also attributed to the the reduced viscous losses associated with the inboard blade sections operating at higher Reynolds numbers due to the larger chord lengths. 


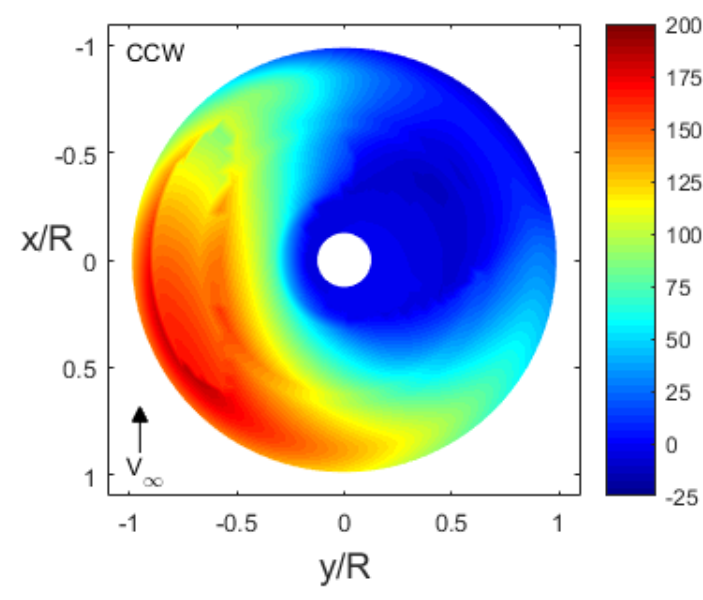

(a) Design 1 (3 blades)

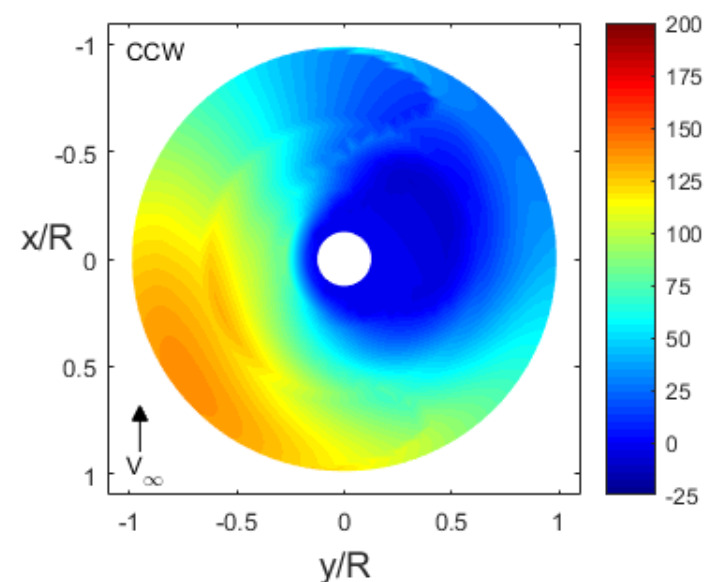

(b) Design 2 (2 blades)

Figure 6.14: Section thrust per unit span, $\frac{d T}{d r}\left[\frac{N}{m}\right]$

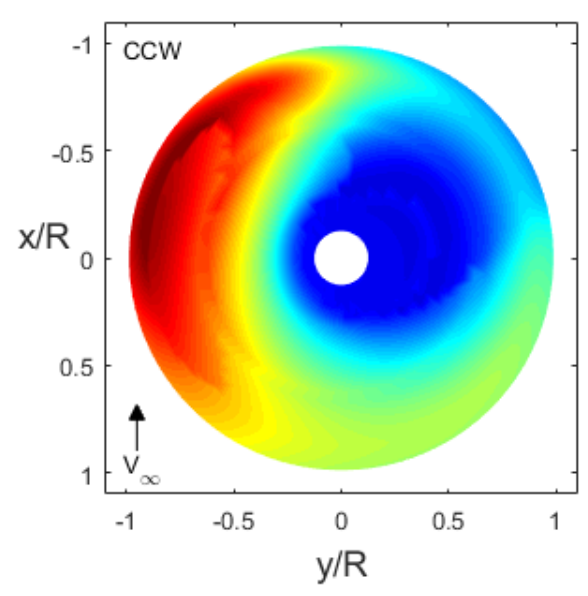

(a) Design 1 (3 blades)

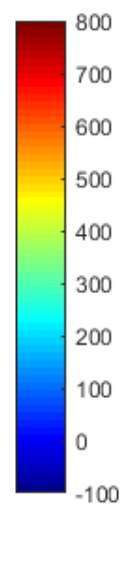

Figure 6.15: Section power per unit span, $\frac{d P}{d r}\left[\frac{W}{m}\right]$

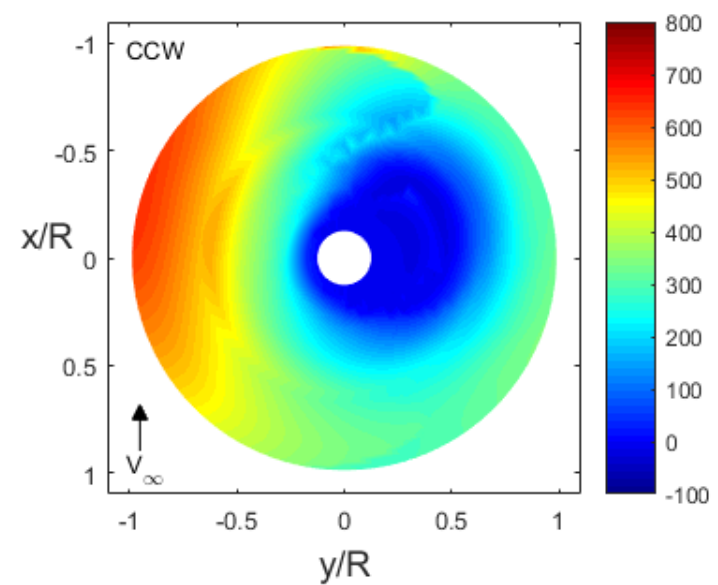

(b) Design 2 (2 blades) 


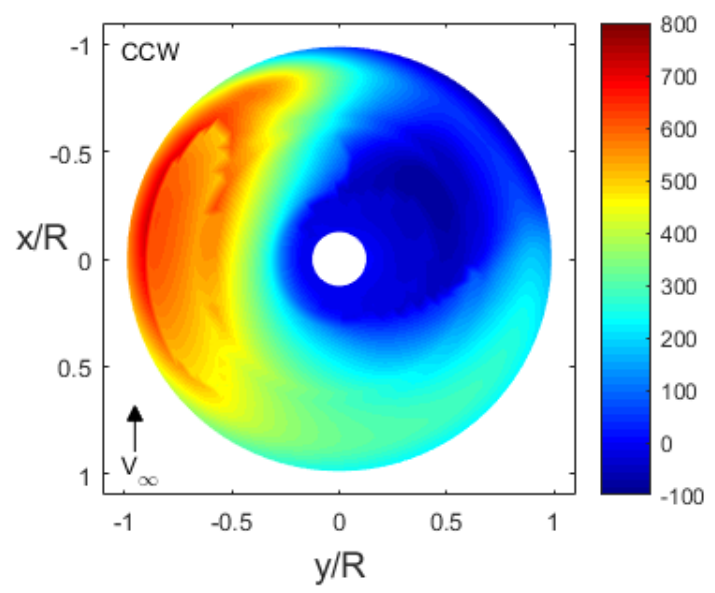

(a) Design 1 (3 blades)

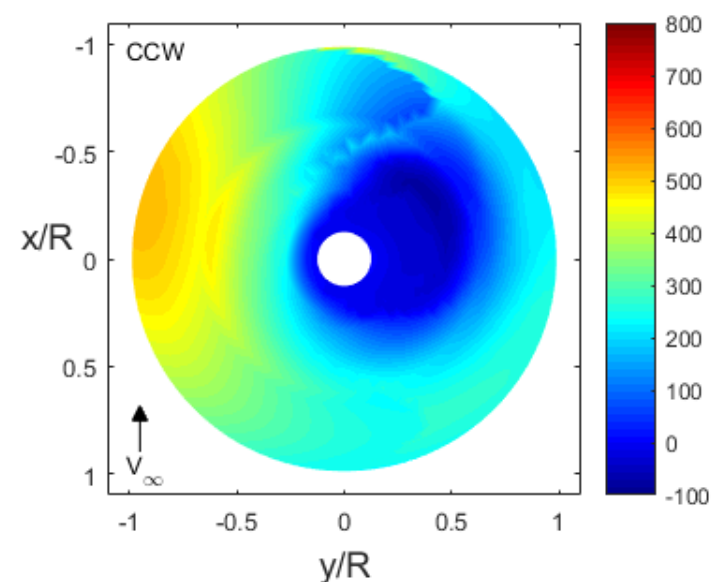

(b) Design 2 (2 blades)

Figure 6.16: Section induced power per unit span, $\frac{d P_{i}}{d r}\left[\frac{W}{m}\right]$

Referring to Table 6.9, the multi-point designs show an approximately even compromise between performances at the single operating points for both the two- and three-bladed multi-point designs. The largest factor affecting these differences, is the compromise in rotational speeds between the two operating points which was slightly increased for each operating condition. The single point designs from the previous single-point cases are also evaluated with respect to their multi-point objective performance and are listed in Table 6.10. The reduced performance of the single-point objective designs, that have power loadings which range between $4.8-30 \%$ lower, demonstrates the importance of including multiple objectives in the design scheme.

\begin{tabular}{|c|c|c|}
\hline Design & $\frac{T}{P}$ for Hover [N/W] & $\frac{T}{P}$ for Cruise [N/W] \\
\hline \hline Baseline & 0.21 & 0.19 \\
\hline Multi-point design 1 & 0.23 & 0.19 \\
\hline Multi-point design 2 & 0.22 & 0.20 \\
\hline
\end{tabular}

Table 6.9: Single point performance for the multi-point designs. 


\begin{tabular}{|c|c|c|}
\hline Design & Multi-point $\frac{T}{P}[\mathbf{N} / \mathbf{W}]$ & Off-point $\frac{T}{P}[\mathbf{N} / \mathbf{W}]$ \\
\hline \hline Hover Design 1 & 0.20 & 0.1924 \\
\hline Hover Design 2 & 0.17 & 0.1754 \\
\hline Forward Flight Design & 0.20 & 0.1850 \\
\hline
\end{tabular}

Table 6.10: Performance of the single-point designs for the multi-point and off-point objectives.

\subsection{Modelling the Wake of a Quad-rotor}

Using the example sUAV platform and operating parameters that are described in Sec.6.3, the velocities that are induced from adjacent rotors are investigated in this section. The purpose of this exercise is to explore whether the wake induced velocities acting in the normal and in-plane directions constitute a significant portion of the rotor's inflow and also to highlight some of the challenges associated with prediction the rotor performance of configured rotors.

The normalized velocities induced by the adjacent rotors are shown in Figs.6.17 through 6.34 for several skew angles and two rotor orientations. These skew angles encompass the edgewise flight capabilities of the representative example vehicle in the previous section, that produces a wake skew angle of $51.8^{\circ}$ in cruise. The rotors for this example are separated by a distance of $0.25 R$ and the interactions between the rotor wakes and the vehicle body and support arms are neglected. The calculated induced velocities are normalized with respect to the rotor's self-induced velocity, which is approximated as being uniform, and is an assumption that largely holds for lightly loaded blades. The induced velocities are oriented with respect to the global coordinate system reference frame.

In hover, the wake induced velocities that are normal to rotor plane are less than $8 \%$ of the rotor's self-induced velocities for most of the rotor disk area. As the rotor wakes progressively become more swept back, portions of the rotor disk, especially those which are located directly downstream from other rotors, are subject to wake induced velocities that have a magnitude of 5 to $10 \%$ of the rotor's self induced velocity. Conversely, rotors that operate directly adjacent to another rotors wake encounter an upwash. This includes a large portion of the forward-most rotor, which for skew angles exceeding $45^{\circ}$, has an upwash component that is approximately 
$10 \%$ of the self-induced velocity for a large portion of its disk area. The in-plane velocities, though remaining relatively constant in the y-direction for most skew angles, decrease in the $\mathrm{x}$-direction as the skew angle increases. For these low skew angle cases, a large portion of the rotor disk operates with lateral induced velocities exceeding 10 to $20 \%$ and therefore should be also considered when modelling the inflow of adjacent hovering rotors. These examples demonstrate the importance of including the wake interference velocities when modelling the complete vehicle performance. 


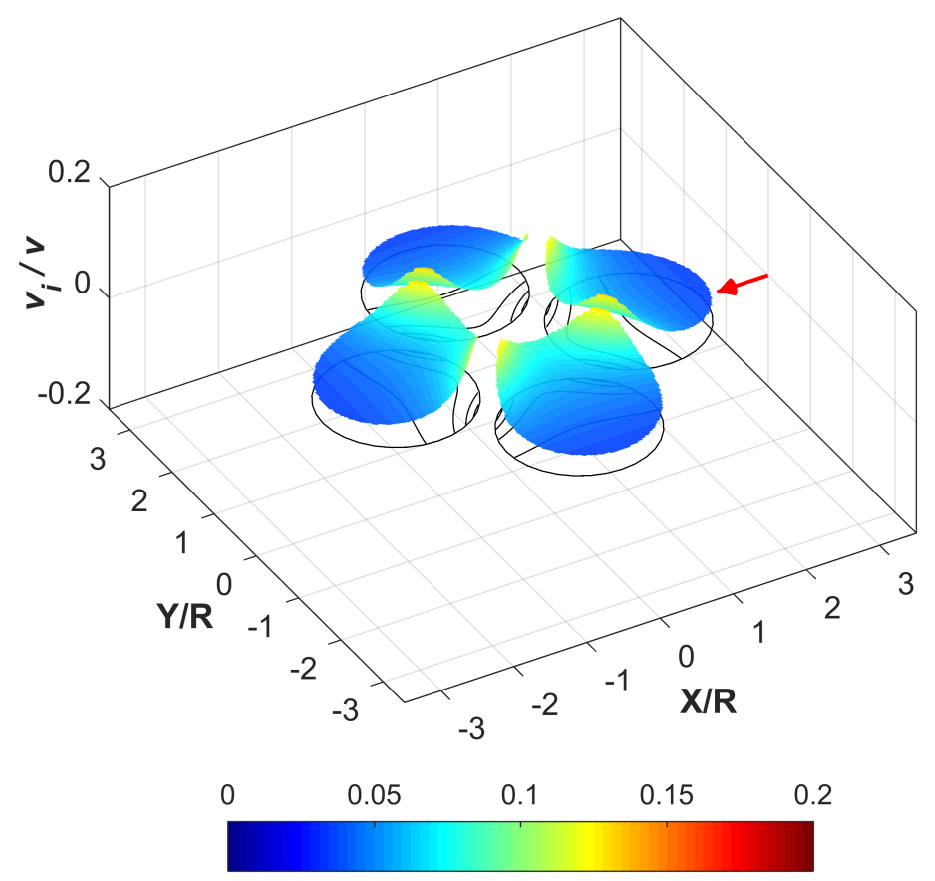

Figure 6.17: Induced velocity ratio, $\frac{v_{i}}{v}$, from adjacent rotors for $\chi=0^{\circ}$ (diamond).

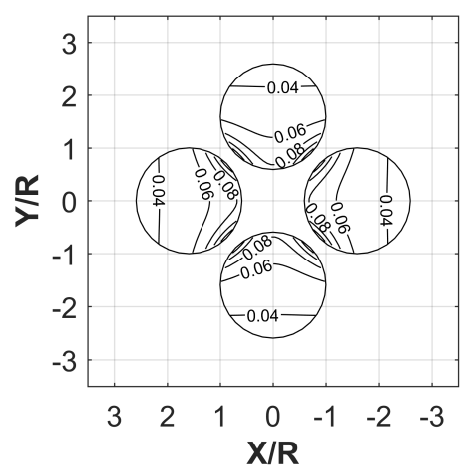

(a) $\frac{V_{i, z}}{v}$

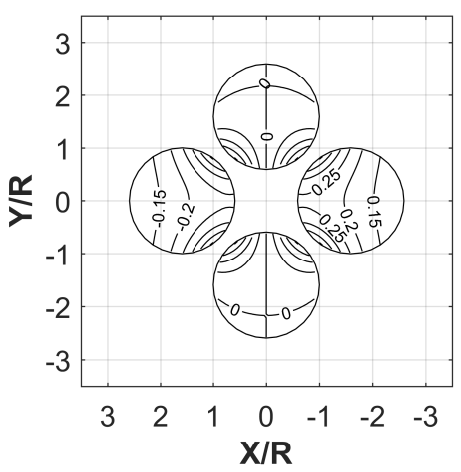

(b) $\frac{V_{i, x}}{v}$

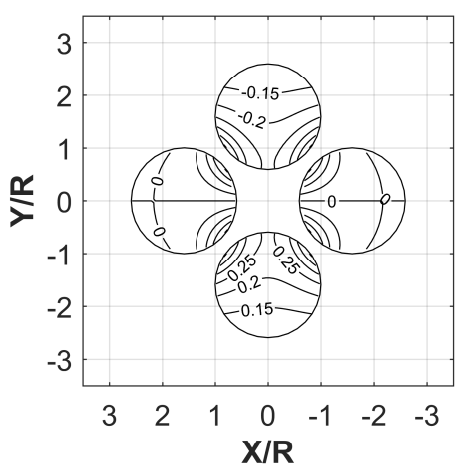

(c) $\frac{V_{i, y}}{v}$

Figure 6.18: Normalized induced velocity ratio contours at the rotor plane for $\chi=0^{\circ}$ for the diamond configuration. 


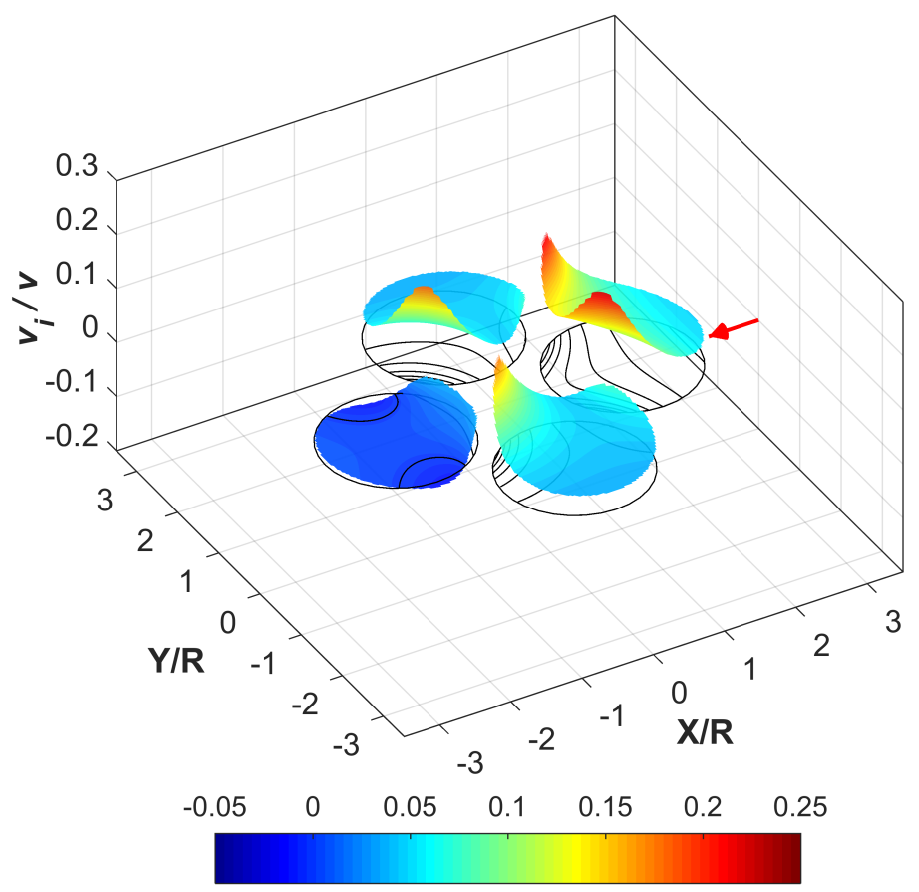

Figure 6.19: Induced velocity ratio, $\frac{v_{i}}{v}$, from adjacent rotors for $\chi=$ $14.04^{\circ}$ (diamond).

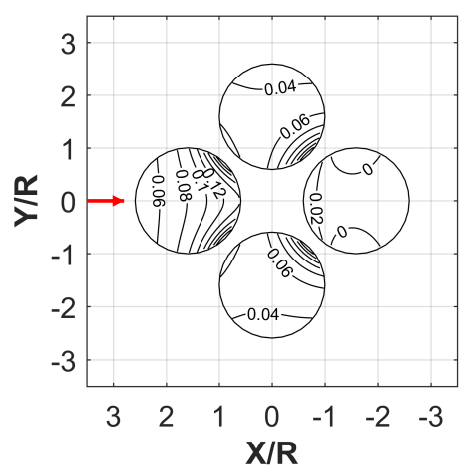

(a) $\frac{V_{i, z}}{v}$

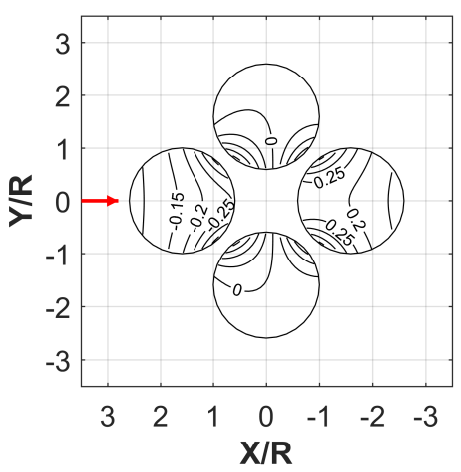

(b) $\frac{V_{i, x}}{v}$

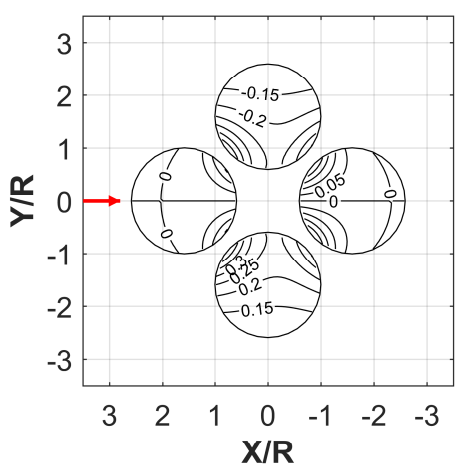

(c) $\frac{V_{i, y}}{v}$

Figure 6.20: Normalized induced velocity ratio contours at the rotor plane for $\chi=14.04^{\circ}$ for the diamond configuration. 


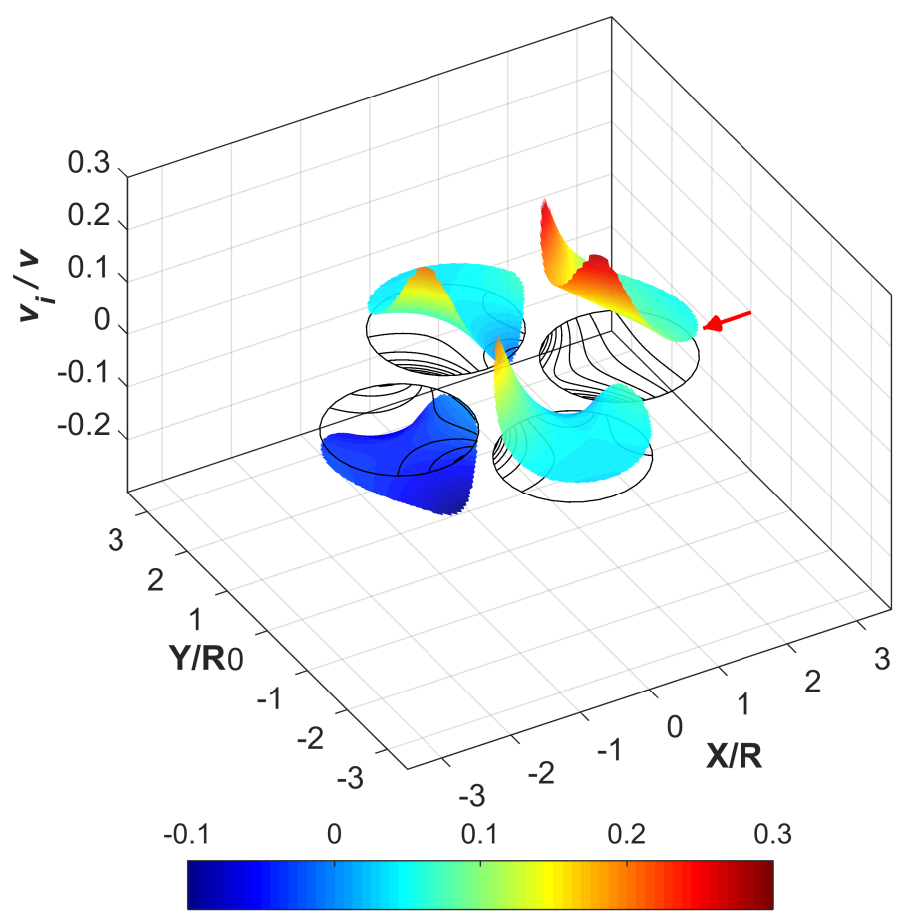

Figure 6.21: Induced velocity ratio, $\frac{v_{i}}{v}$, from adjacent rotors for $\chi=$ $26.56^{\circ}$ (diamond).

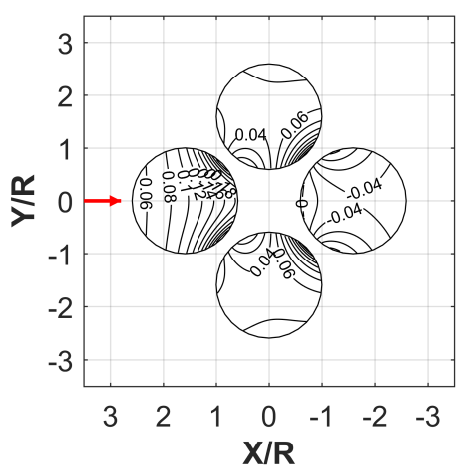

(a) $\frac{V_{i, z}}{v}$

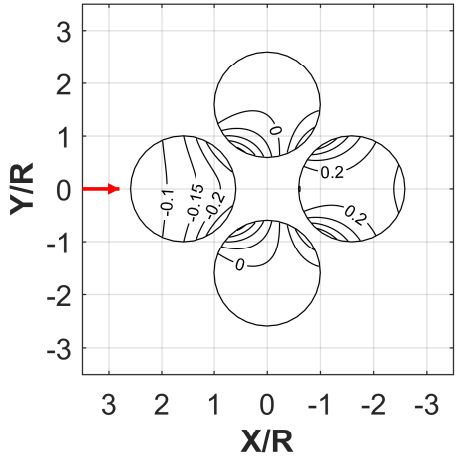

(b) $\frac{V_{i, x}}{v}$

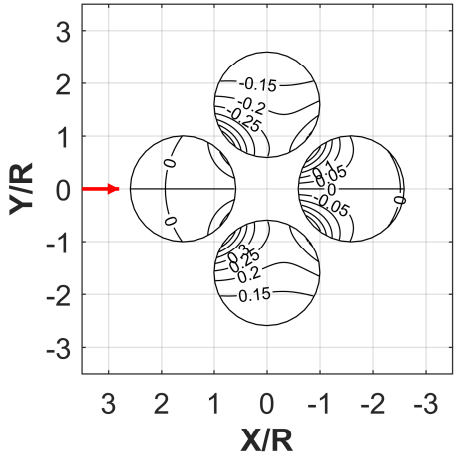

(c) $\frac{V_{i, y}}{v}$

Figure 6.22: Normalized induced velocity ratio contours at the rotor plane for $\chi=26.56^{\circ}$ for the diamond configuration. 


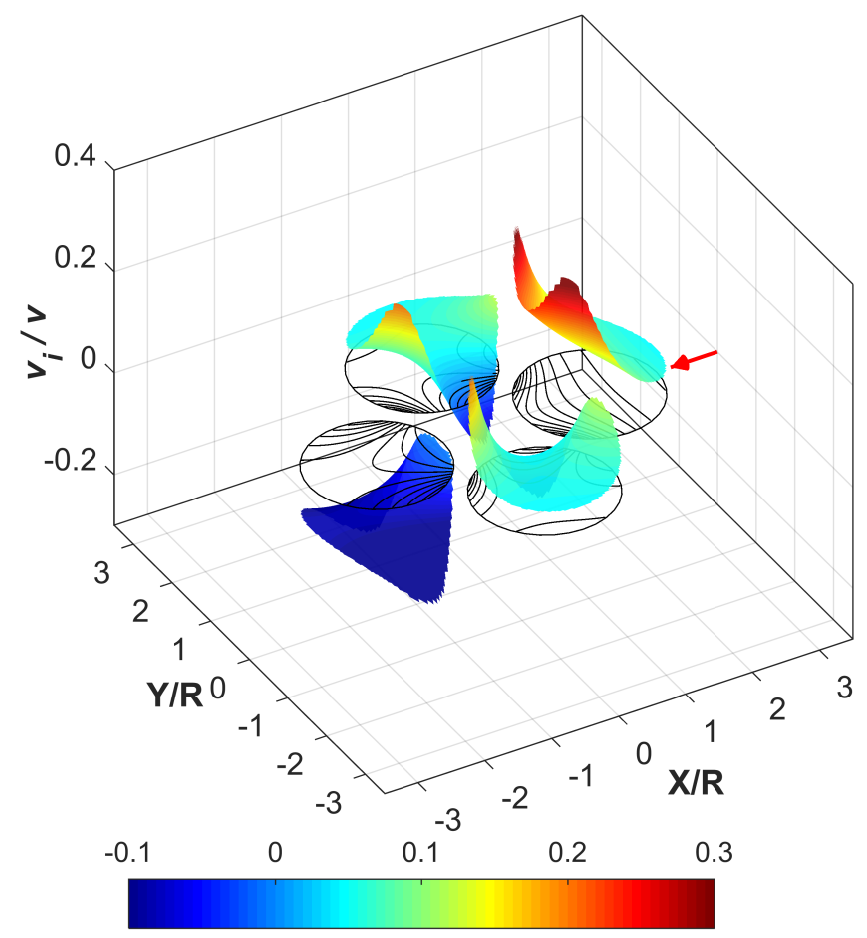

Figure 6.23: Induced velocity ratio, $\frac{v_{i}}{v}$, from adjacent rotors for $\chi=45^{\circ}$ (diamond).

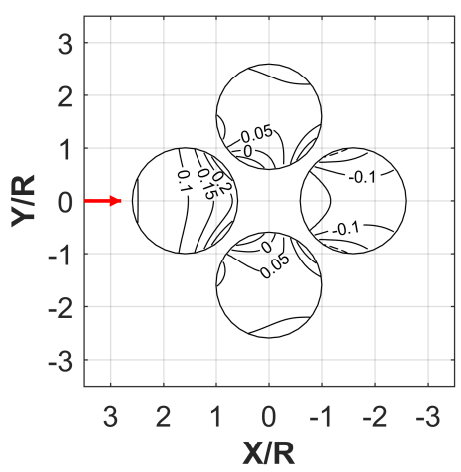

(a) $\frac{V_{i, z}}{v}$

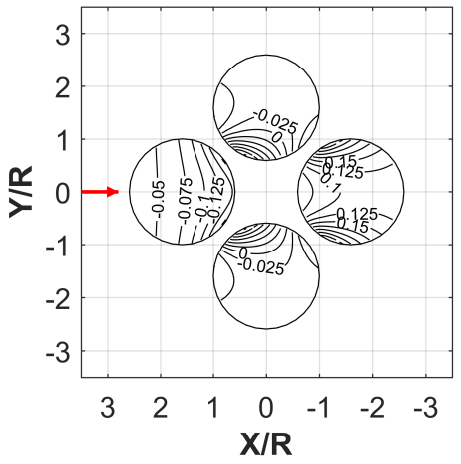

(b) $\frac{V_{i, x}}{v}$

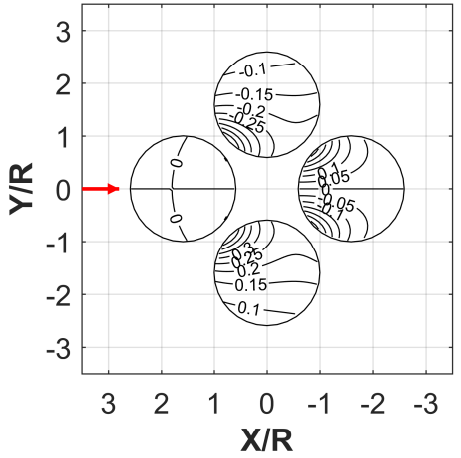

(c) $\frac{V_{i, y}}{v}$

Figure 6.24: Normalized induced velocity ratio contours at the rotor plane for $\chi=45^{\circ}$ for the diamond configuration. 


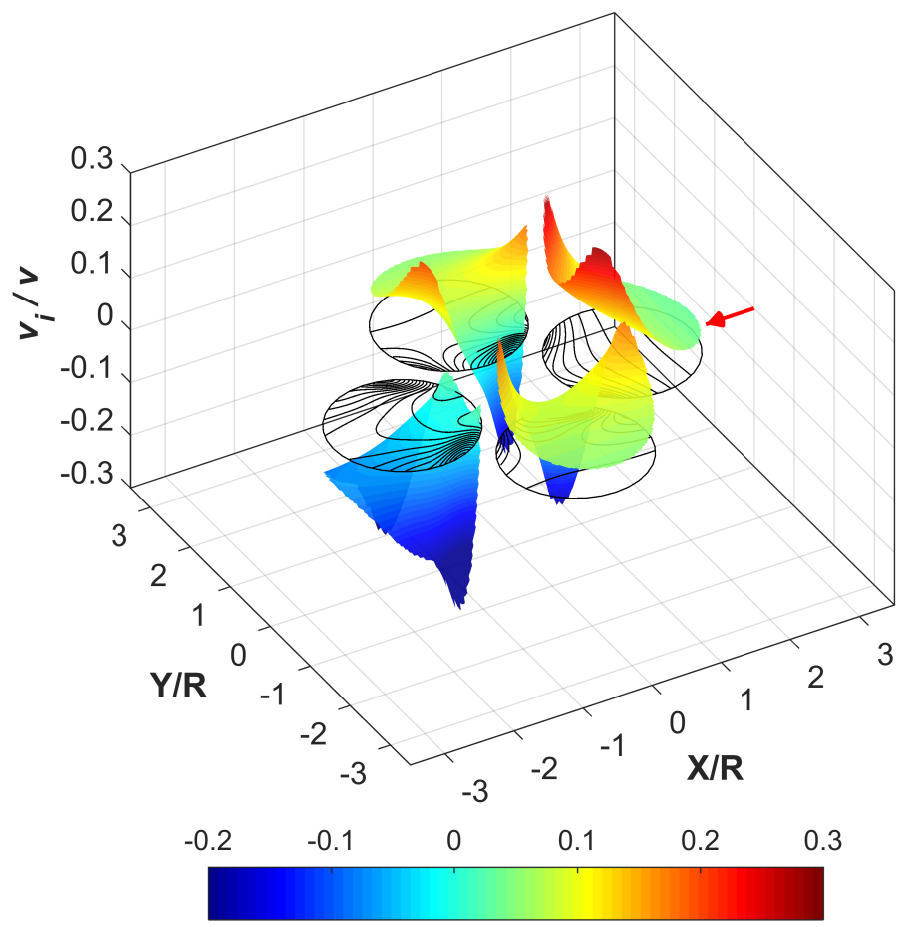

Figure 6.25: Induced velocity ratio, $\frac{v_{i}}{v}$, from adjacent rotors for $\chi=$ $63.43^{\circ}$ (diamond).

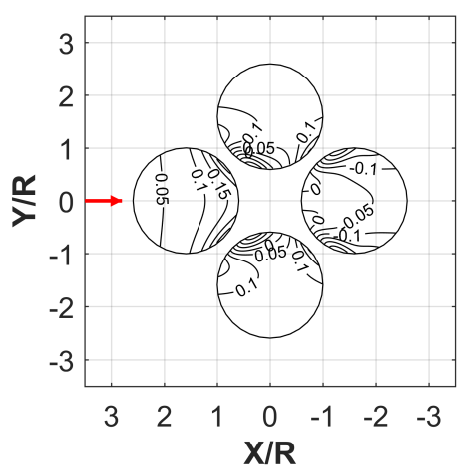

(a) $\frac{V_{i, z}}{v}$

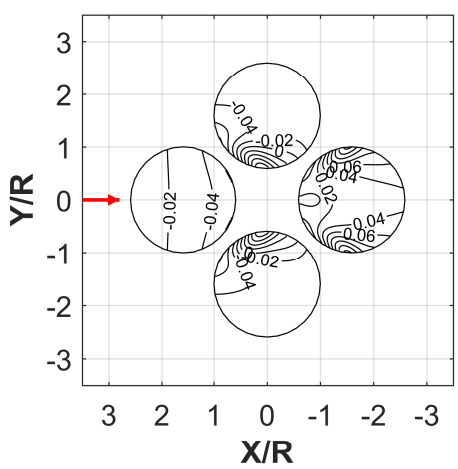

(b) $\frac{V_{i, x}}{v}$

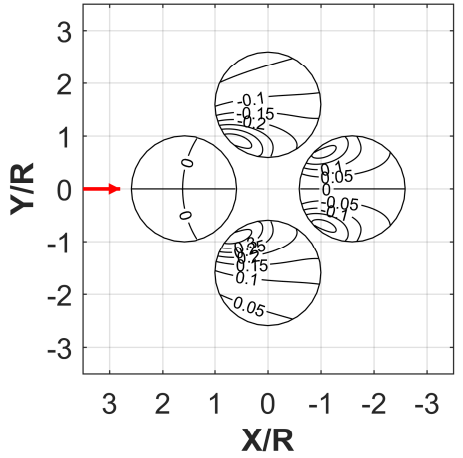

(c) $\frac{V_{i, y}}{v}$

Figure 6.26: Normalized induced velocity ratio contours at the rotor plane for $\chi=63.43^{\circ}$ for the diamond configuration. 


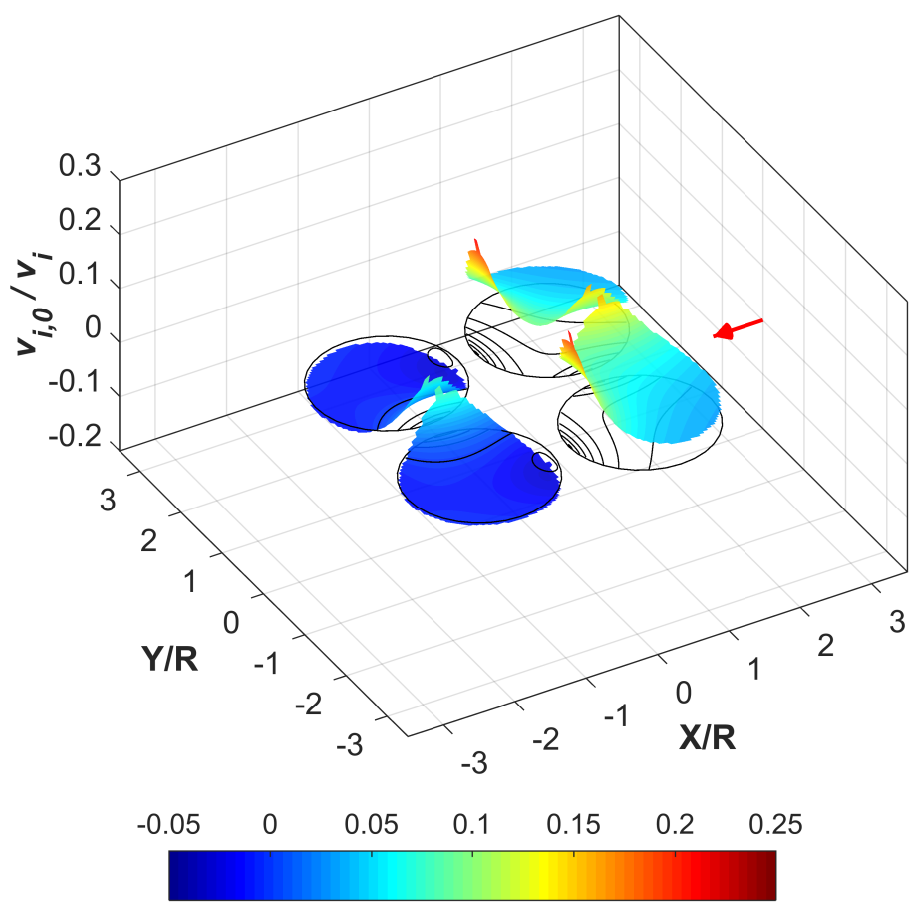

Figure 6.27: Induced velocity ratio, $\frac{v_{i}}{v}$, from adjacent rotors for $\chi=$ $14.04^{\circ}$ (square).

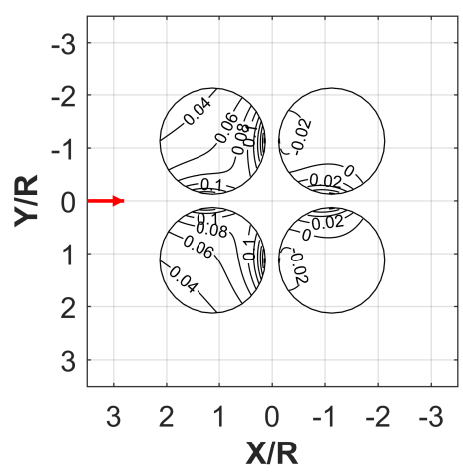

(a) $\frac{V_{i, z}}{v}$

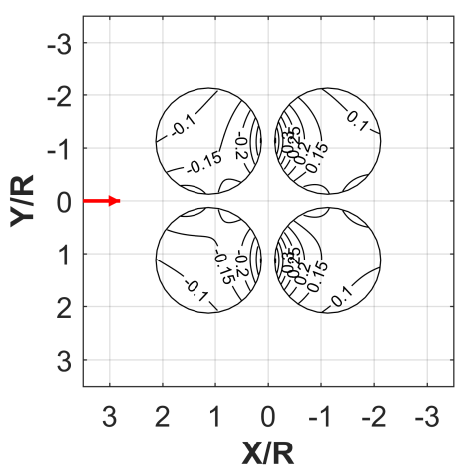

(b) $\frac{V_{i, x}}{v}$

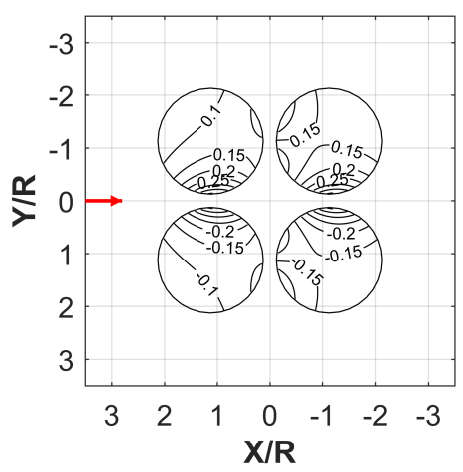

(c) $\frac{V_{i, y}}{v}$

Figure 6.28: Normalized induced velocity ratio contours at the rotor plane for $\chi=14.04^{\circ}$ for the square configuration. 


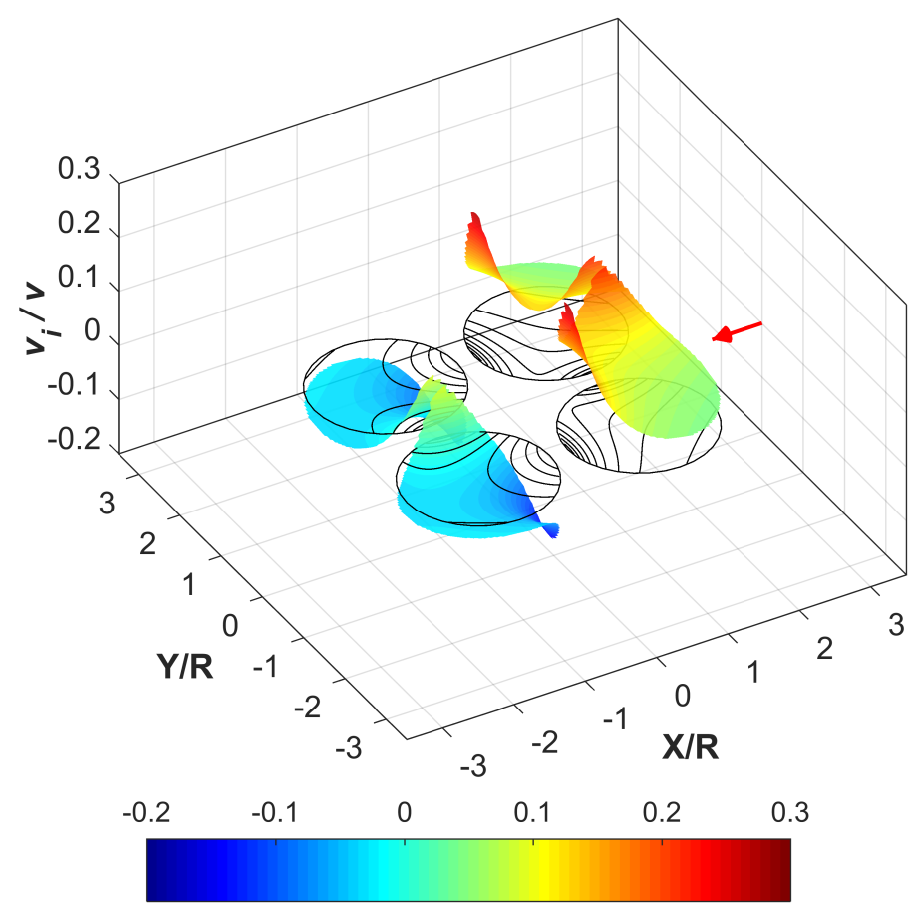

Figure 6.29: Induced velocity ratio, $\frac{v_{i}}{v}$, from adjacent rotors for $\chi=$ $26.56^{\circ}$ (square).

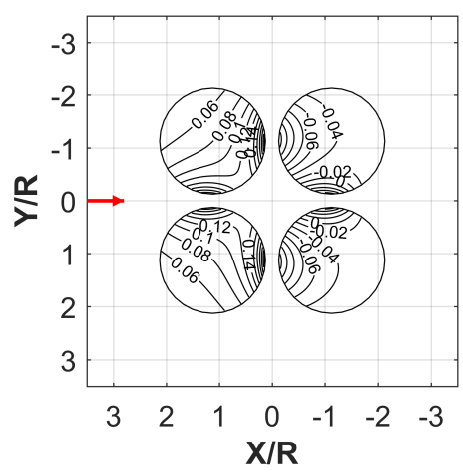

(a) $\frac{V_{i, z}}{v}$

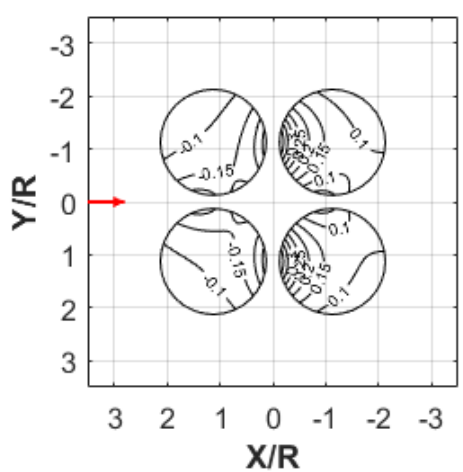

(b) $\frac{V_{i, x}}{v}$

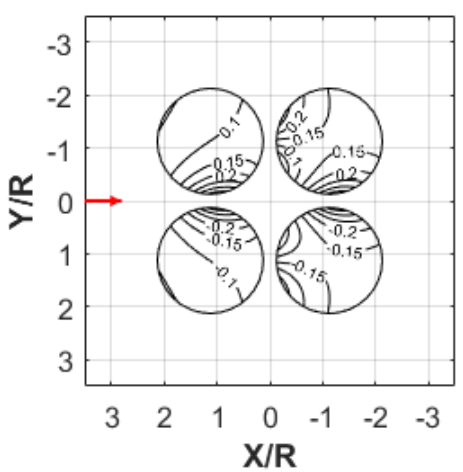

(c) $\frac{V_{i, y}}{v}$

Figure 6.30: Normalized induced velocity ratio contours at the rotor plane for $\chi=26.56^{\circ}$ for the square configuration. 


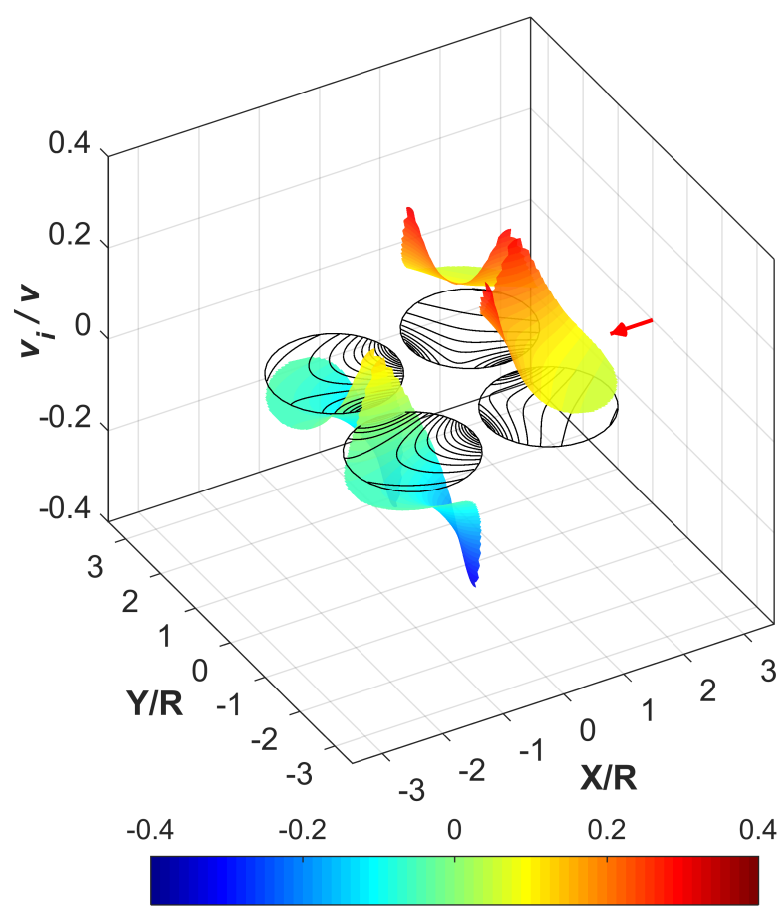

Figure 6.31: Induced velocity ratio, $\frac{v_{i}}{v}$, from adjacent rotors for $\chi=45^{\circ}$ (square).

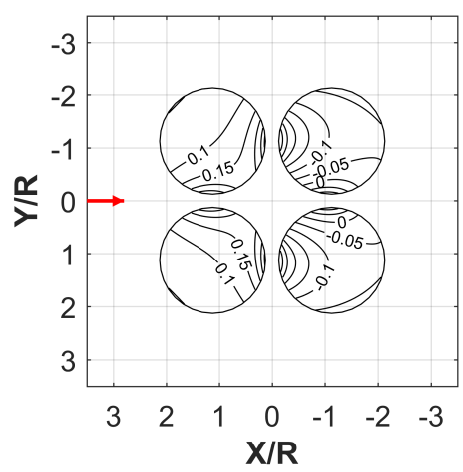

(a) $\frac{V_{i, z}}{v}$

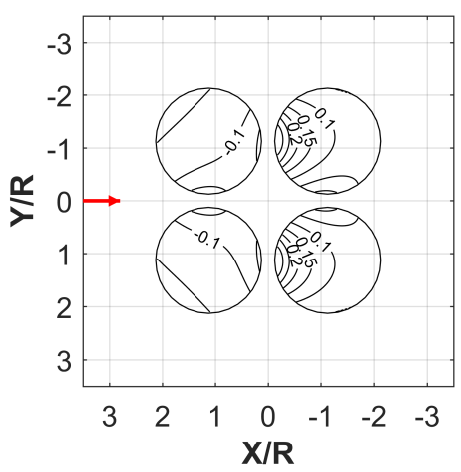

(b) $\frac{V_{i, x}}{v}$

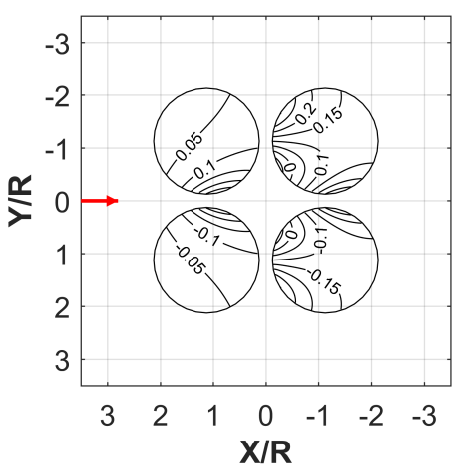

(c) $\frac{V_{i, y}}{v}$

Figure 6.32: Normalized induced velocity ratio contours at the rotor plane for $\chi=45^{\circ}$ for the square configuration. 


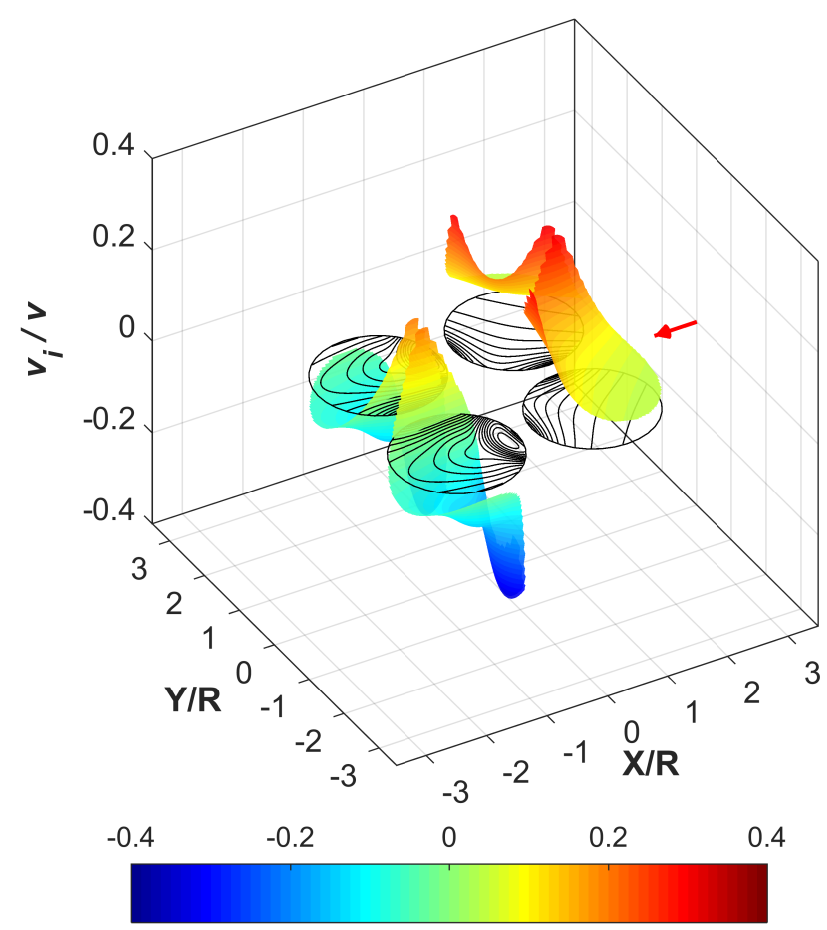

Figure 6.33: Induced velocity ratio, $\frac{v_{i}}{v}$, from adjacent rotors for $\chi=$ $63.43^{\circ}$ (square).

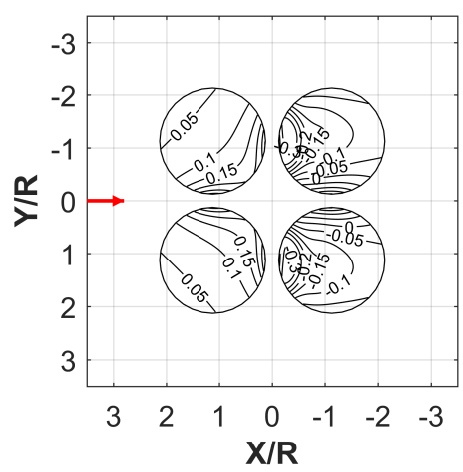

(a) $\frac{V_{i, z}}{v}$

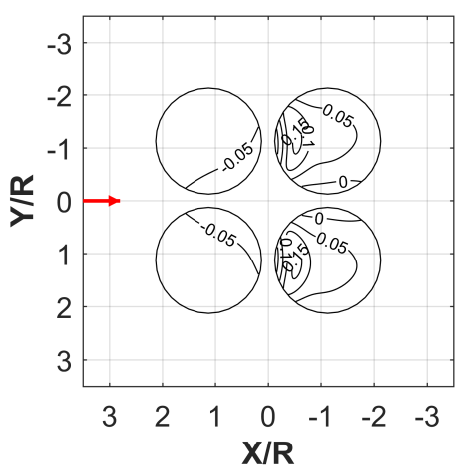

(b) $\frac{V_{i, x}}{v}$

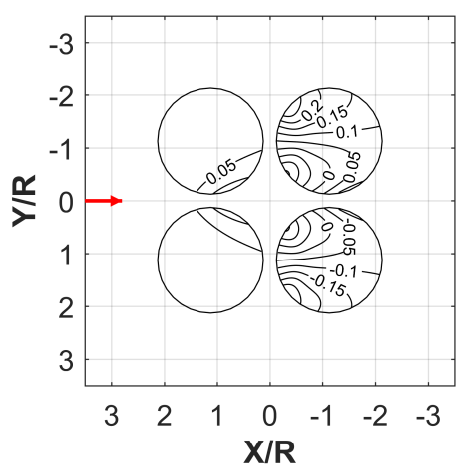

(c) $\frac{V_{i, y}}{v}$

Figure 6.34: Normalized induced velocity ratio contours at the rotor plane for $\chi=63.43^{\circ}$ for the square configuration. 


\section{Chapter 7}

\section{Conclusions}

\subsection{Conclusions}

A rotor aerodynamic performance prediction model, testing apparatus and design methodology are introduced in order to be used in support of a design-build-test stream for the rotors of small unmanned aerial vehicles. Considerable effort was applied to modelling the rotor's performance characteristics across several operating conditions, particularly in edgewise flight, and developing an associated design methodology that encompasses these conditions. A wake interaction model that uses a potential flow approach, is also presented for the purpose of modelling the induced wake effects that adjacent rotor wakes have on the rotor inflow. Both models are implemented into a computer program that predicts the rotor performance and wake geometries of configured vehicles, given a number of input operating conditions.

The rotor performance model is based on a blade element momentum theory approach that is formulated specifically for small rotors. The method considers retreating blade effects and uses an empirical post-stall aerodynamic model. Multiple approaches are presented for modelling the rotor inflow that consist of methods for hover and axial flight that are formulated with and without small angle approximations, and a method for edgewise flight that uses the first harmonic linear inflow models.

One of the more significant aspects of this research is that the predictions of edgewise flight performance are validated against experimental measurements to a more comprehensive degree 
than to what has been published in the existing literature. To generate these experimental results, a variable pitch rotor test stand was built and used to measure the performance of a commercially available rotor at several advance ratios and inflow angles. The thrust predictions show good agreement with measurements for both positive and negative inflow angles. A large portion of the thrust predictions fall within 5 to $15 \%$ of measurements for the inflow angles that are within the cruise capabilities of current day small multirotor vehicles. The predicted power, despite a constant under-prediction of approximately 10 to $20 \%$, agrees with the trends observed for measurements for inflow angles that range from fully edgewise to fully axial flows, excluding the negative inflow angles. Part of this difference was due to systematic errors associated with the method in which power is measured using the test stand. For both thrust and power, differences between measurements and the predictions decrease with increasing blade section Reynolds numbers. One of the largest difficulties associated with predicting the power of small rotors is determining the viscous drag of sections that operate near to, or in, stall. For hover, correctly capturing blade-section characteristics, that is, the drag and lift coefficients, has a larger effect on the accuracy of the performance predictions compared to using inflow models that include swirl velocities. Similar thrust and power predictions were obtained using the approaches that assume uniform inflow and radial inflow distributions.

The rotor performance prediction model is implemented into a design scheme that uses a numerical optimization approach. The objective of the design scheme is to determine blade geometries that satisfy the performance objectives associated with single- and multi-point operating conditions. The optimization approach uses a nested optimization algorithm, that consists of a rotational speed trimming routine as part of the objective function. The combined optimization algorithm and rotor-prediction model methodology is validated using the classical analytical case for the ideal twist required to minimize induced losses for a hovering rotor. Both a genetic and particle swarm algorithm were used to ensure that the method generates results that are reproducible with different solvers.

Designs for several rotor geometries that operate at single and multiple operating conditions are presented. The objective of these designs is to minimize the power loading for a given rotor thrust requirement. The operating conditions consisted of hover and edgewise flight at 
speeds coinciding with the vehicle's cruise speed. For all of the designs, a reduction in the rotational speed, and subsequent increase in solidity, is the primary factor for improving the power loading. All of the designs, including the one for forward flight, have radial twist distributions that resemble the idealized hyperbolic twist distribution for the ideal hovering rotor. Although, the rotor designed for hover has approximately 4 degrees of additional collective pitch. Comparing the two- and three-bladed designs for the multi-point case, rotors having fewer blades and larger sectional chords perform marginally better than the blades with more blades. However, designs where the reduction in rotational speed is achieved by adding more blades also perform marginally better compared to the baseline rotor. To compromise for the multiple flight requirements, the multi-point designs operate at slightly higher rotational speeds in hover than the single-point designs. For comparison sake, the two-bladed multi-point design resembled the geometry of the baseline commercially available two-bladed rotor, but had a slightly larger planform and operated at a $15 \%$ reduced tip-speed. For the forward flight operating condition, the reduction in power between the optimized and baseline designs primary came by reducing the induced power on the retreating blade, and by re-distributing the thrust slightly towards the inboard portion of the blade. In some cases, this comes at the expense of a slight increase in the profile drag on the inboard blade sections. The performance of these designs suggest that the limits of reducing the tip-speed for small multirotor vehicle rotors may be driven by the low-speed efficiency limits of the motor, rather than by the rotor's tip-speed requirements in edgewise flight.

Finally, the rotor wake model is used to demonstrate the effects that the adjacent rotor wakes have on a rotor's inflow. The wake induced velocity distributions resulting from a quadconfigured rotor arrangement for several skew angles are presented. The adjacent wakes have a small effect on the vertical inflow of the surrounding rotors in hover, for which the normal component of the wake interference velocities are between 4 to $8 \%$ of the rotor's self-induced velocity for a large portion of the rotor disk. As the wakes become progressively more skewed, the magnitude of the wake induced velocities that are normal to the rotor disk increase to being 10 to $15 \%$ of the self-induced velocity for portions of the rotor that are positioned either up- or down-stream from an adjacent wake. The in-plane velocities are also determined and show that 
for increasing skew angle, the lateral induced velocities remain relatively constant whereas the longitudinal velocities steadily decrease.

\subsection{Future Work}

There are a number of lessons learned from this thesis that are discussed here and provide a foundation for which future work can be based:

- The validation of the rotor performance model in edgewise flight showed that the model inaccurately predicts the required power for inflow angles that are less than 0 degrees, including those that correspond with the vortex ring state. This is caused by more complicated inflow distributions that are affected by the interactions between discrete features in the wake and other effects such as blade-vortex interactions. In these instances, momentum theory is substituted with either empirical trends derived from experimentation or more advanced wake models. Therefore, either a more comprehensive wake models, such as the free-vortex wake method, or experimental trends determined from more small rotor testing are needed to improve the prediction capabilities of the model.

- There is also a need to extend the validation efforts to the additional forces and moments generated by the rotor blades in edgewise flight. The trimming forces required for the vehicle to remain stable become significantly more pronounced with increasing flight speed, and thus, need to be modelled to capture their influence on the operating and design requirements of the rotor.

- The optimization approach can be extended to other disciplines in order to capture some of the factors peripheral to the aerodynamic aspects of the design. This includes the implementation of structural, aeroelastic and aeroacoustic constraints, in addition to design factors that would affect the vehicles configuration, such as a more advanced vehicle trim model. An immediate example of this is the investigation into using thinner blade sections with respect to the structural requirements of the blade. 
- The design approach would immediately benefit from the inclusion of a brushless motor and battery model that captures the motor efficiency characteristics with changing rotational speed. Implementing such a model allows for the exploration of the lower tip-speed limits associated with maximizing the rotor power loading.

- The numerical optimization approach uses a heuristic method and, therefore, absolute proof that the optimized geometries are global minimums is not guaranteed. Although the computational effort required for running the forward flight cases is relatively expensive, re-running the cases using additional methods, such as the global search with multiple starting points, may help in verifying that a global minimum has been found.

- Using control points to parameterize the blade has the disadvantage that some nearidentical radial chord and twist distributions can be obtained using several control point positions. Other approaches, such as the class-shape-transformation technique, may be useful for reducing the number of iterations required by the optimizer since each shape is unique to a single combination of design variables.

- There are limitations on how the model handles unique features on the blade, specifically ones designed to modify the wake structure. These include tip-vortex generators, dihedral and swept tips, and more advanced hub designs. Although these design features must be investigated using more advanced prediction methods or experimental testing, determining if some of these can be retro-fitted onto an existing blade designs and still provide a performance benefit, despite not being explicitly modelled, warrants investigation. 
Appendices 


\section{Appendix A}

\section{A.1 Force and Moment Coefficients of the Rotor and Propeller Con- ventions}

The relationship between the rotor and propeller coefficients can be found through rearrangement with $\Omega=2 \pi n$, where $\Omega$ is the rotational speed in radians per second and $n$ is the rotational speed in revolutions per second.

$$
\begin{aligned}
C_{T} & =\frac{T}{\rho A(\Omega R)^{2}}=\frac{T}{\rho 4 \pi^{3} n^{2}\left(\frac{D}{2}\right)^{4}}=\left(\frac{16}{4 \pi^{3}}\right) \frac{T}{\rho n^{2} D^{4}} \\
& =\left(\frac{4}{\pi^{3}}\right) C_{T, P} \\
C_{Q} & =\frac{Q}{\rho A \Omega^{2} R^{3}}=\frac{Q}{\rho \pi^{3} 4 n^{2}\left(\frac{D}{2}\right)^{5}}=\left(\frac{32}{4 \pi^{3}}\right) \frac{Q}{\rho n^{2} D^{5}} \\
& =\left(\frac{8}{\pi^{3}}\right) C_{Q, P} \\
C_{P} & =\frac{P}{\rho A(\Omega R)^{3}}=\frac{P}{8 \rho \pi^{4} n^{3}\left(\frac{D}{2}\right)^{5}}=\left(\frac{32}{8 \pi^{4}}\right) \frac{P}{\rho n^{3} D^{5}} \\
& =\left(\frac{4}{\pi^{4}}\right) C_{P, P}
\end{aligned}
$$

Conversion factors in Eq.A.1 and Eq.A.2 also apply, respectively, to the remaining forces and moments shown in Table 3.1.

In propeller theory, the advance ratio, $J$, assumes that the entirety of the inflow is axial and 
is defined as:

$$
J=\frac{V}{n D}
$$

Relating the rotor and propeller advance ratios for the axial inflow condition gives the following relationship:

$$
\mu_{z}=\frac{V_{\infty} \sin \alpha}{\Omega R}=\frac{V_{\infty}}{2 \pi n R}=\frac{1}{\pi} J
$$




\section{A.2 Rotor and Propeller Geometries}

The geometric characteristics of the rotors and propellers tested in this thesis are shown in Figs. A.2.1 and A.2.2 below:

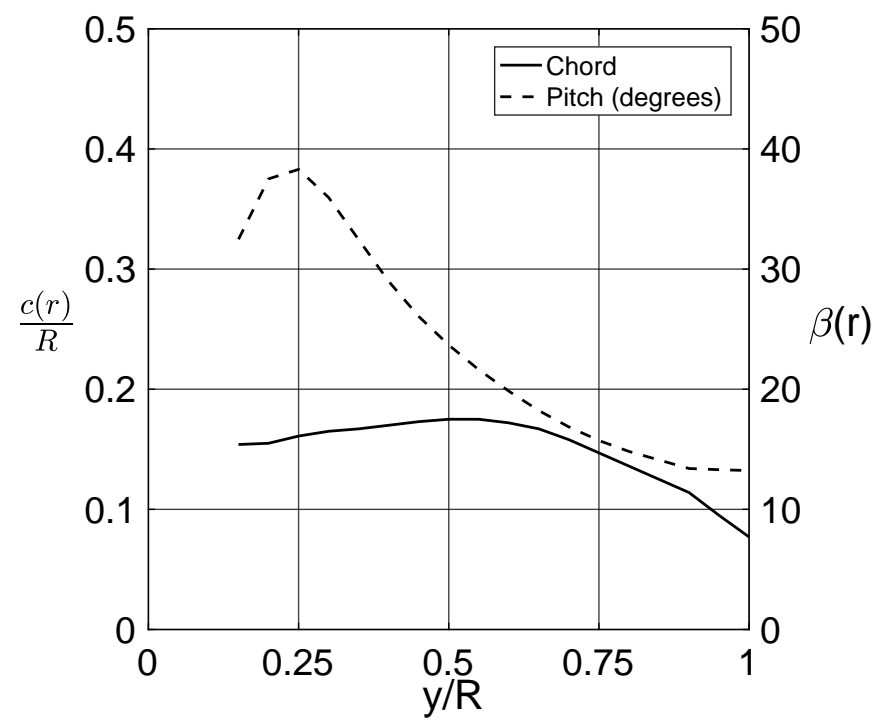

Figure A.2.1: Sectional chord and pitch distributions for the MA11x7E

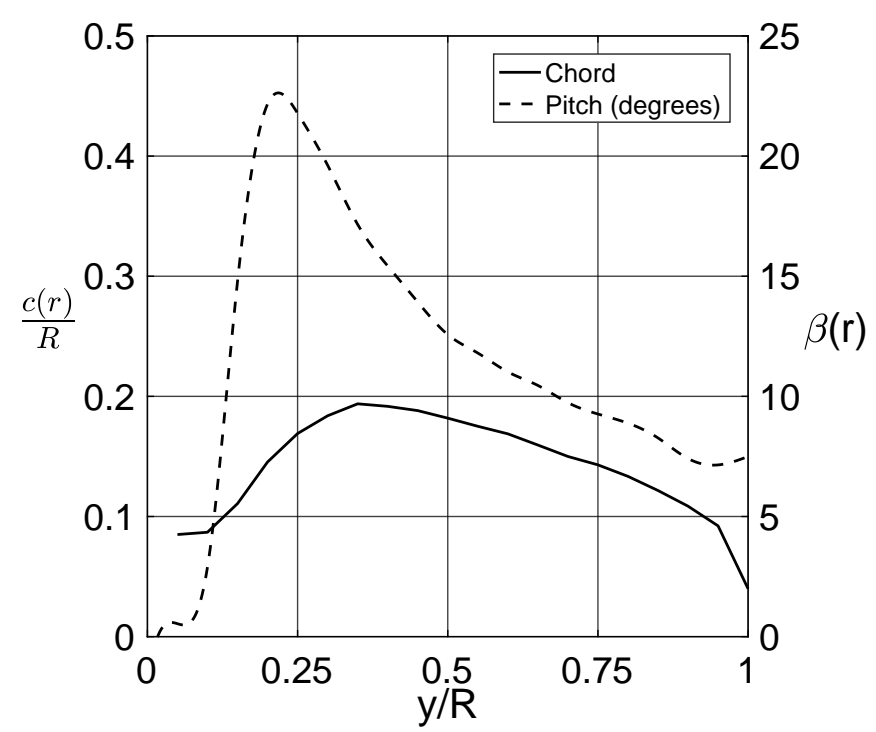

Figure A.2.2: Sectional chord and pitch distributions for the T-motor 18x6.1 


\section{A.3 Rotor Test Data}

This appendix consists of the complete test data generated by the RU test stand for the T-motor $18 \times 6.1$ rotor.

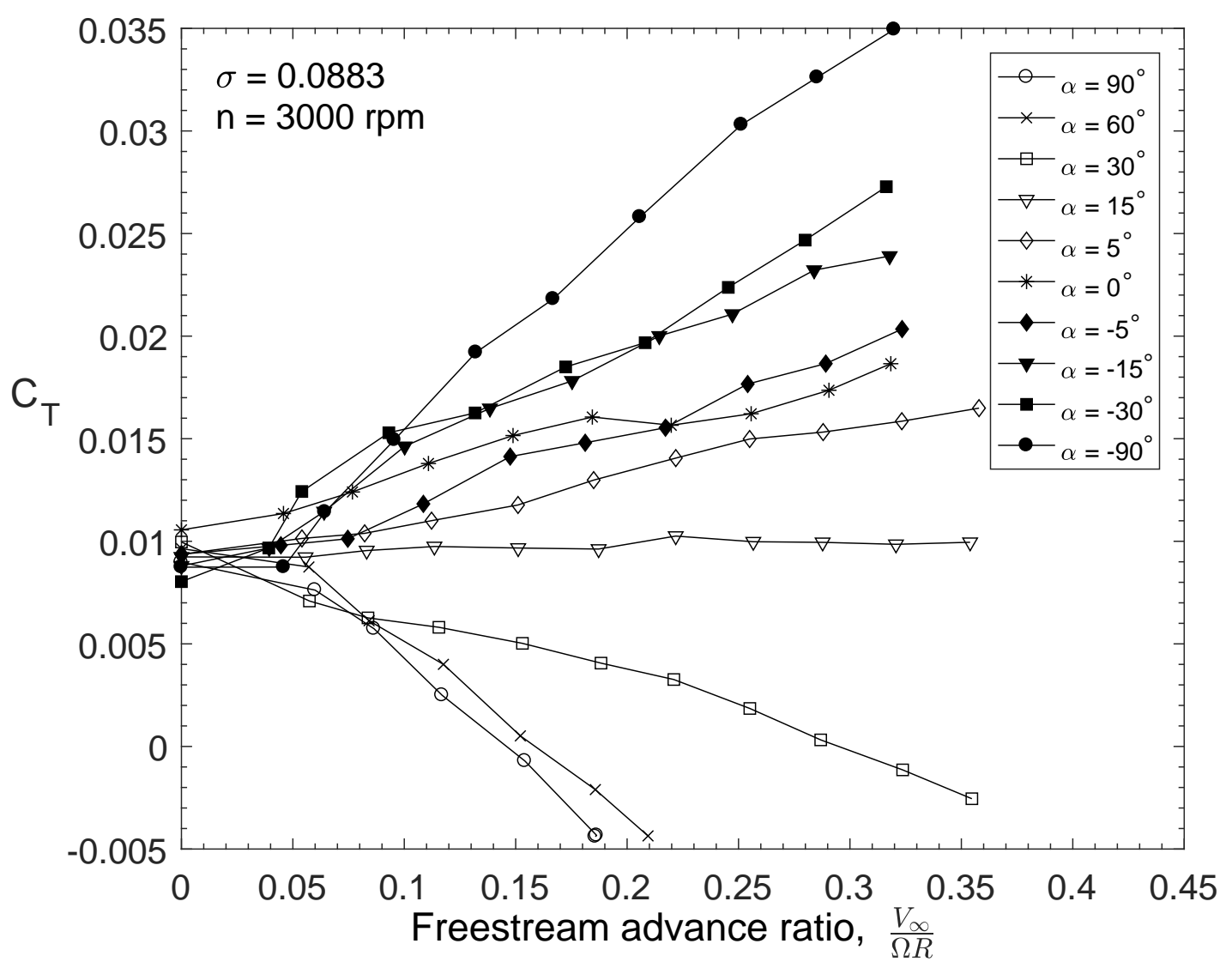

Figure A.3.1: $C_{T}$ versus advance ratio for the T-motor $18 \times 6.1, \mathrm{RPM}=3000$ 


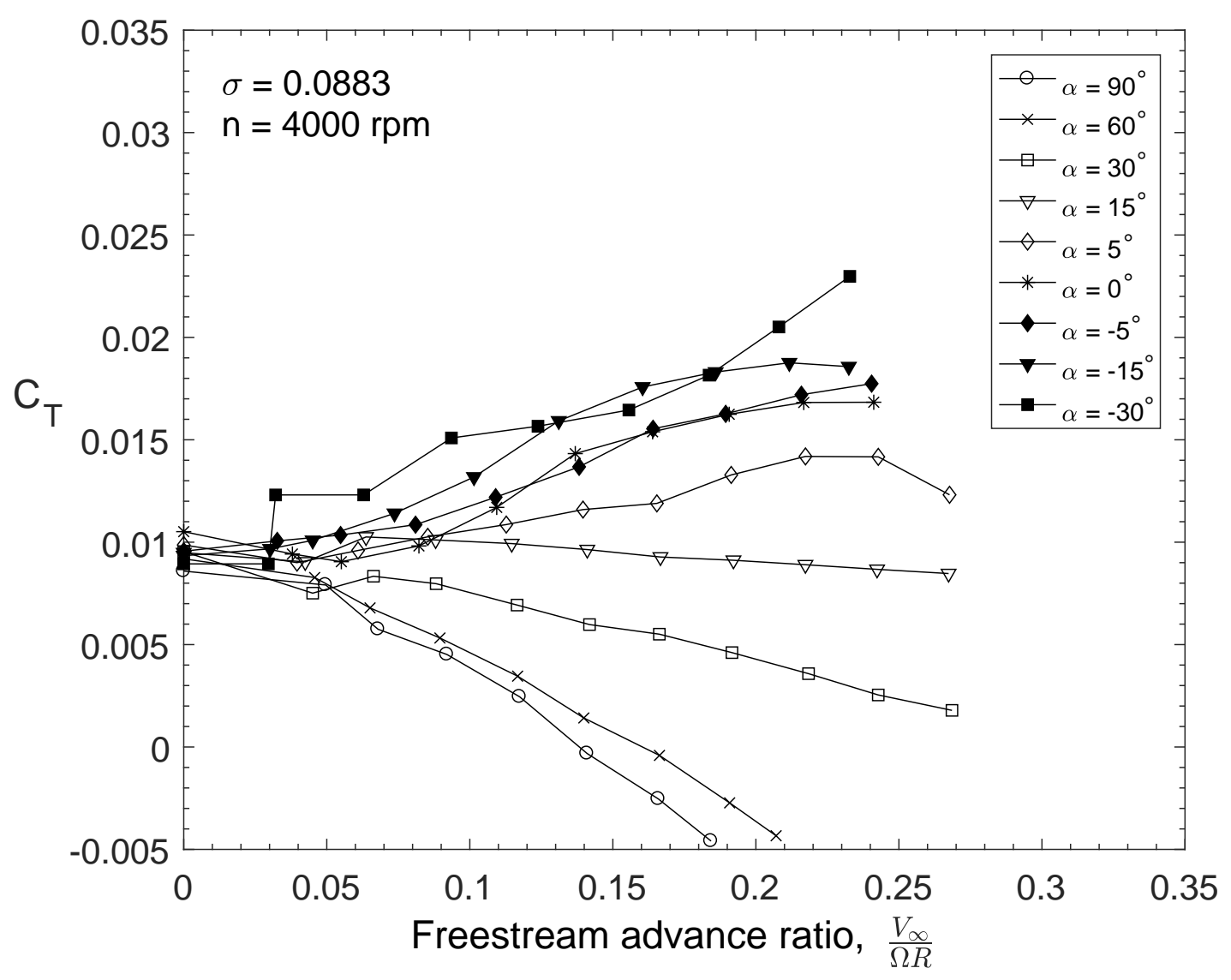

Figure A.3.2: $C_{T}$ versus advance ratio for the T-motor $18 x 6.1, \mathrm{RPM}=4000$ 


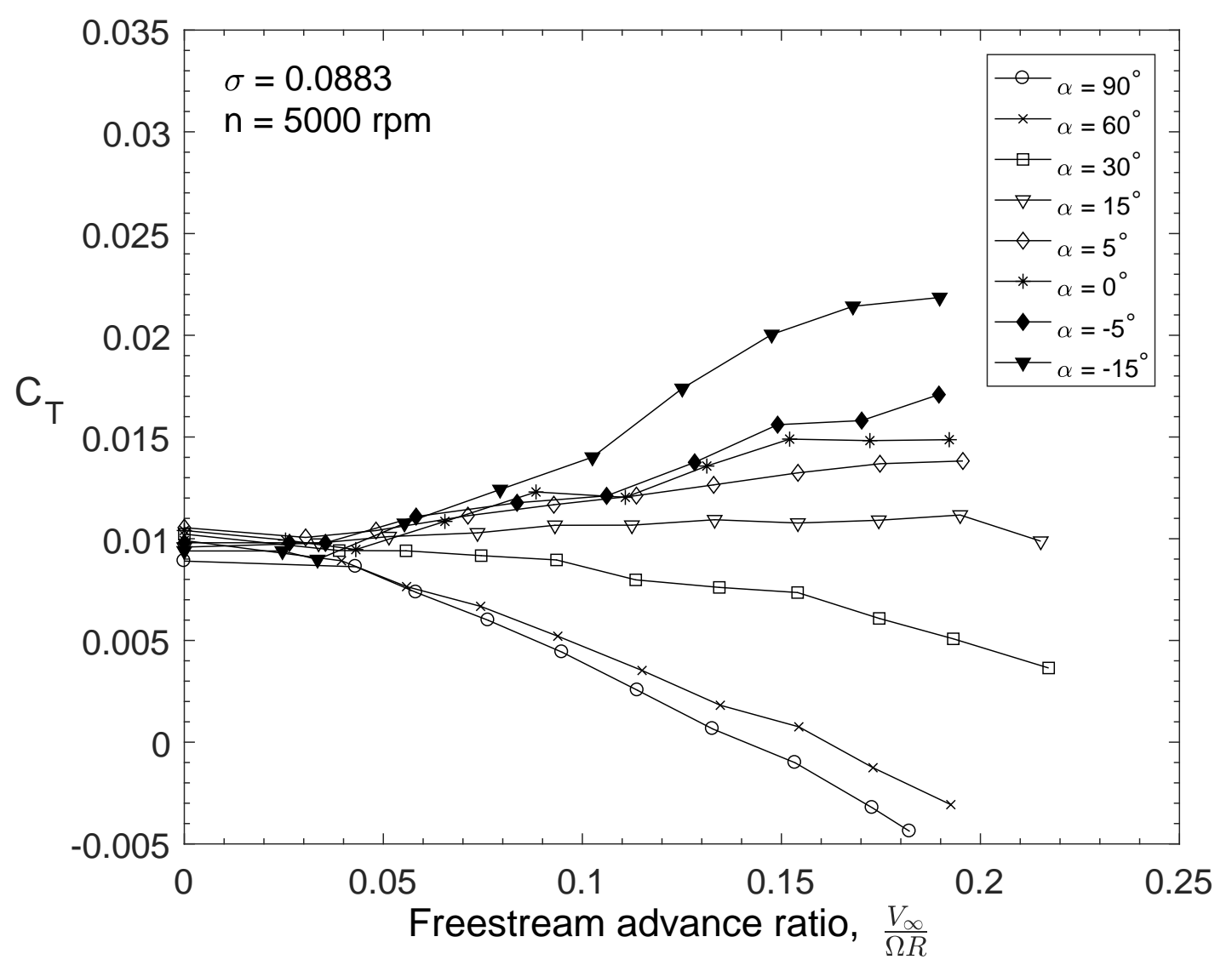

Figure A.3.3: $C_{T}$ versus advance ratio for the T-motor $18 x 6.1, \mathrm{RPM}=5000$ 


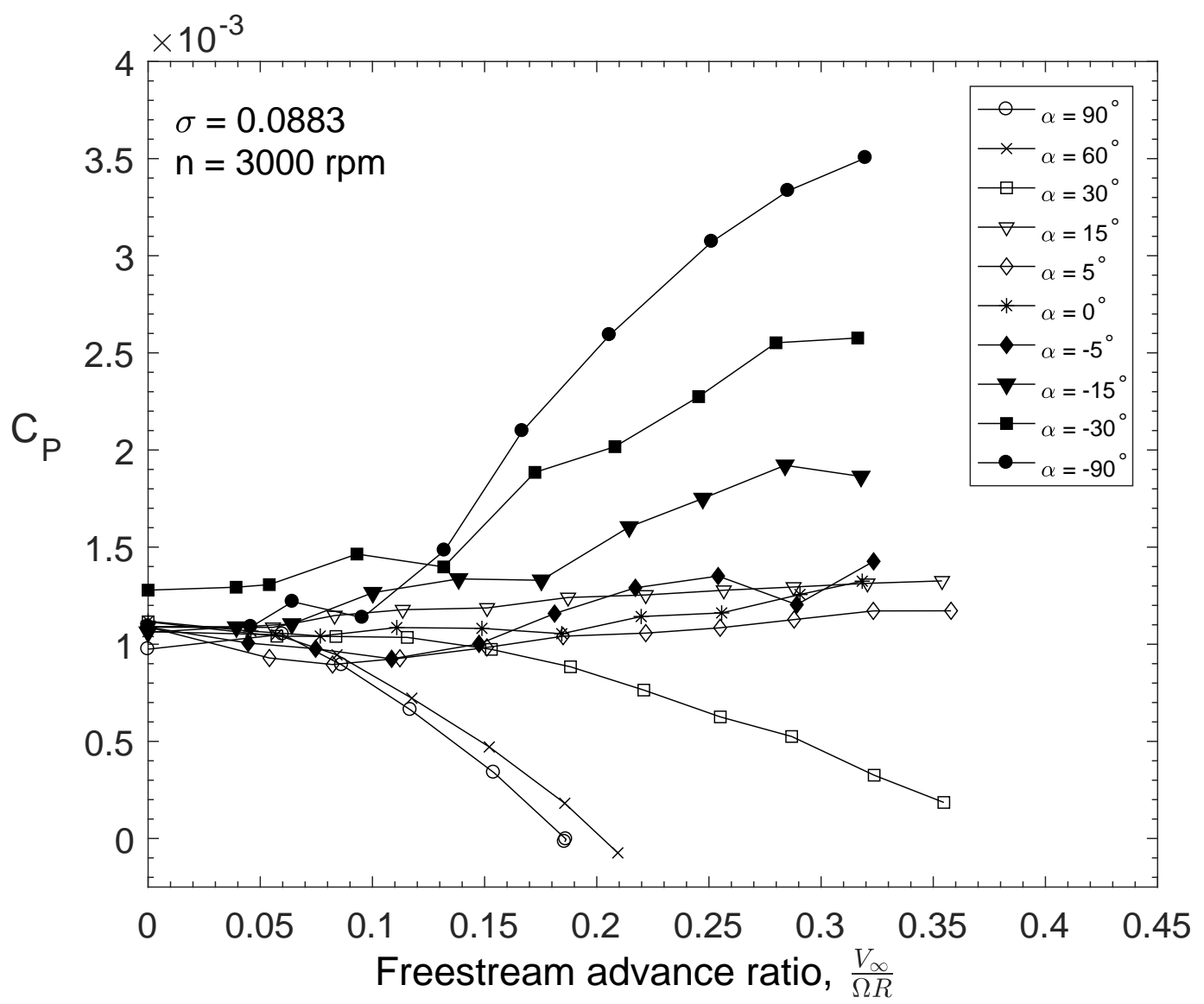

Figure A.3.4: $C_{P}$ versus advance ratio for the T-motor $18 x 6.1, \mathrm{RPM}=3000$ 


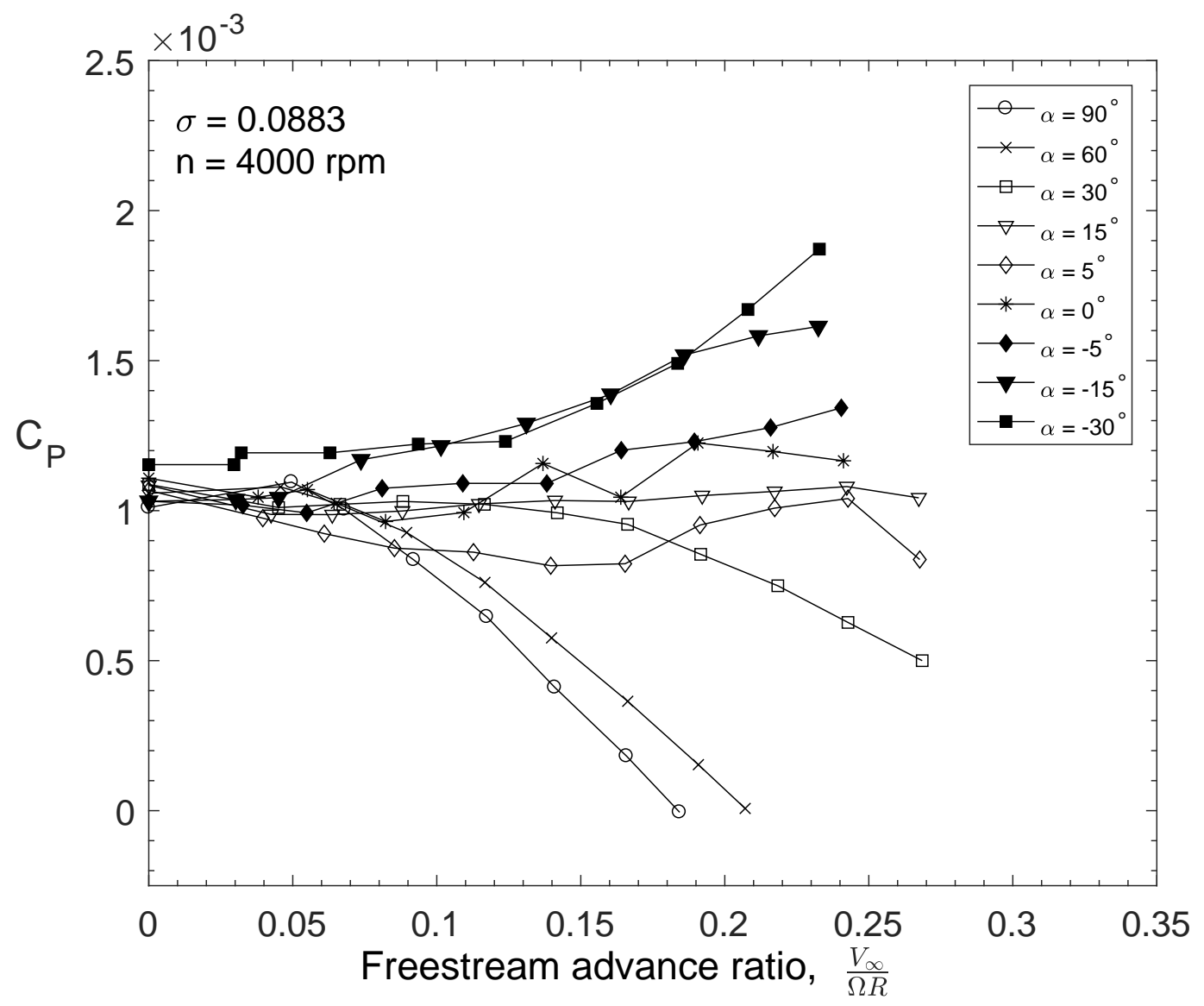

Figure A.3.5: $C_{P}$ versus advance ratio for the T-motor $18 x 6.1, \mathrm{RPM}=4000$ 


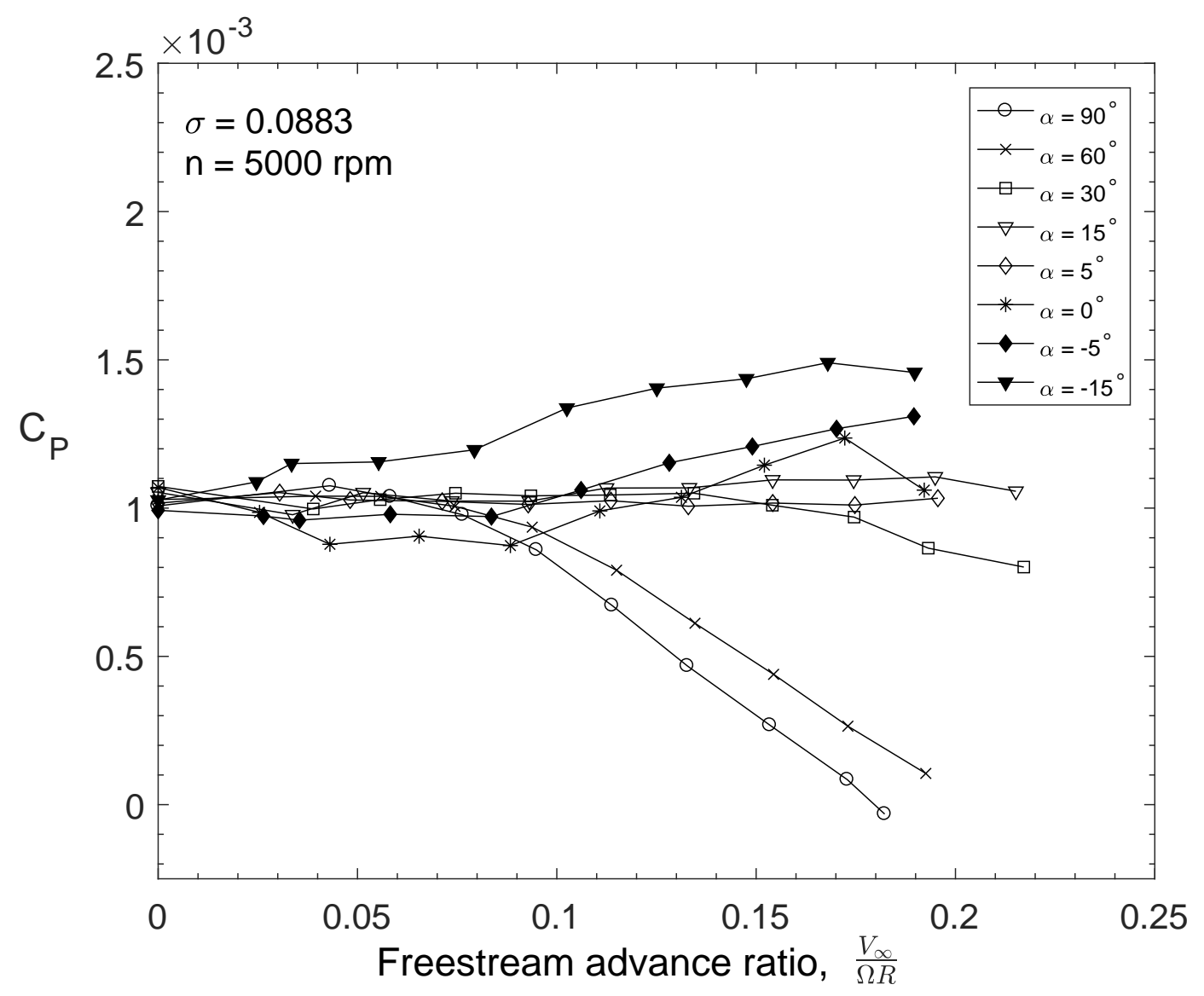

Figure A.3.6: $C_{P}$ versus advance ratio for the T-motor $18 x 6.1, \mathrm{RPM}=5000$ 


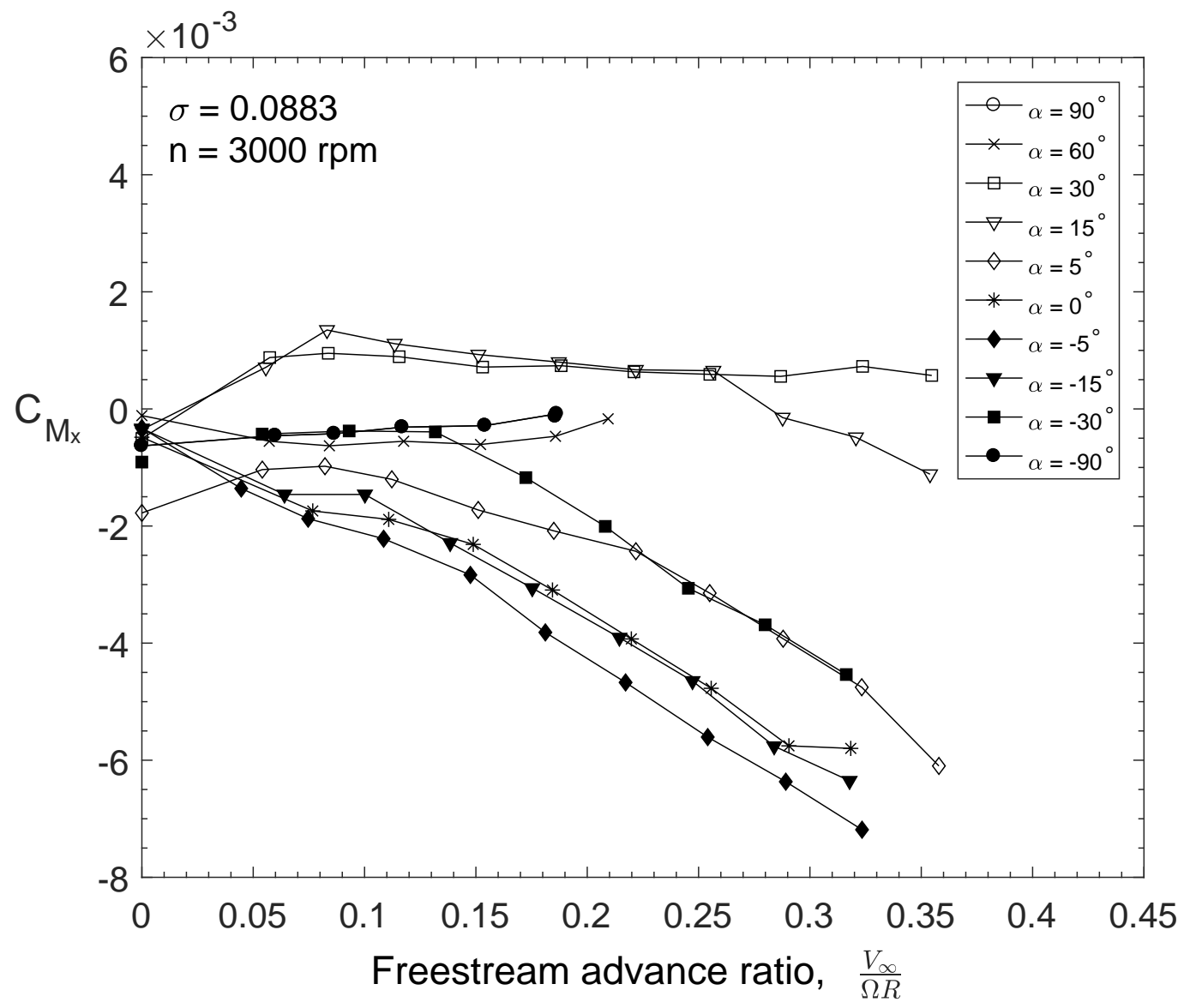

Figure A.3.7: $C_{M_{x}}$ versus advance ratio for the T-motor 18x6.1, RPM $=3000$ 


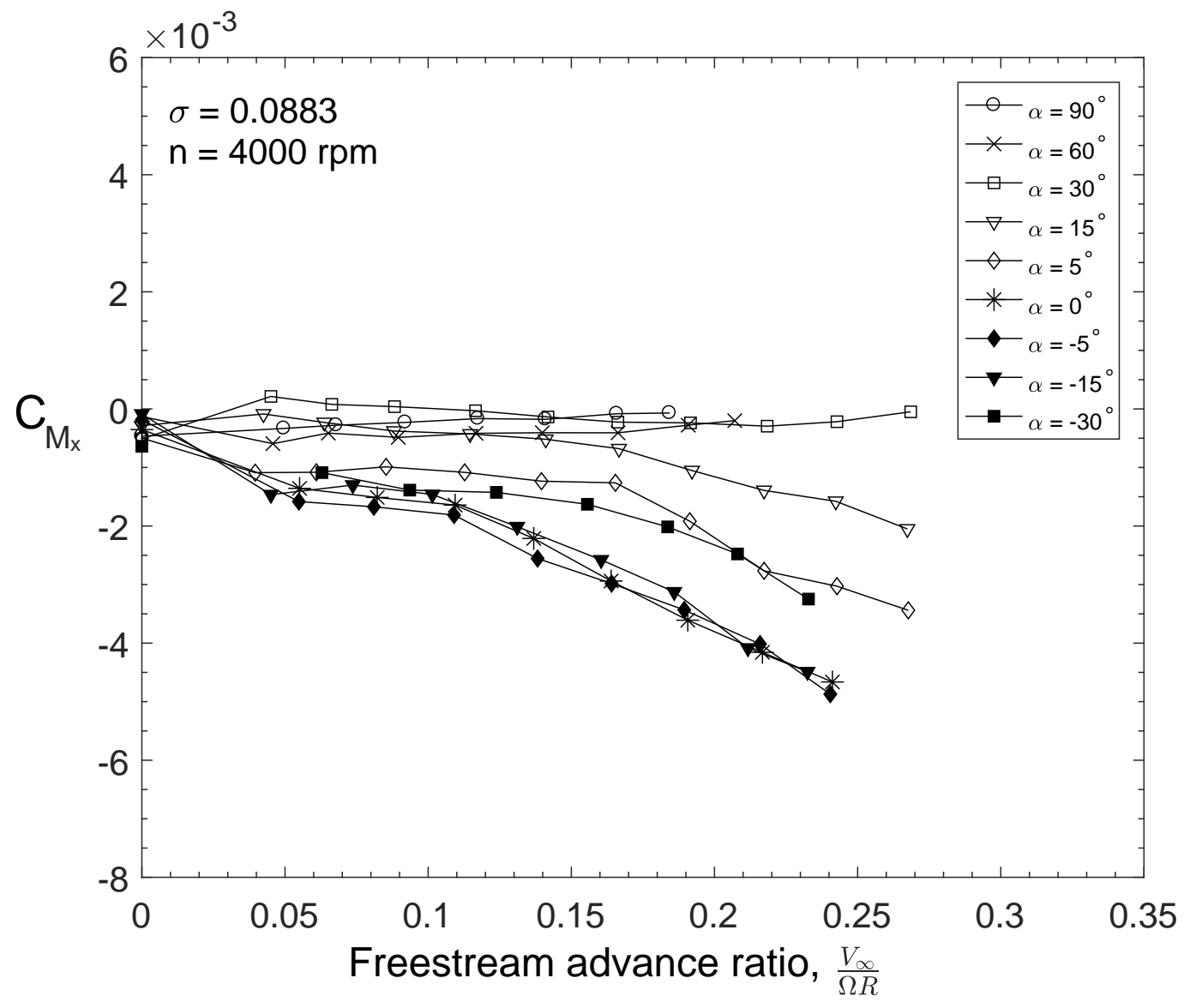

Figure A.3.8: $C_{M_{x}}$ versus advance ratio for the T-motor 18x6.1, RPM $=4000$ 


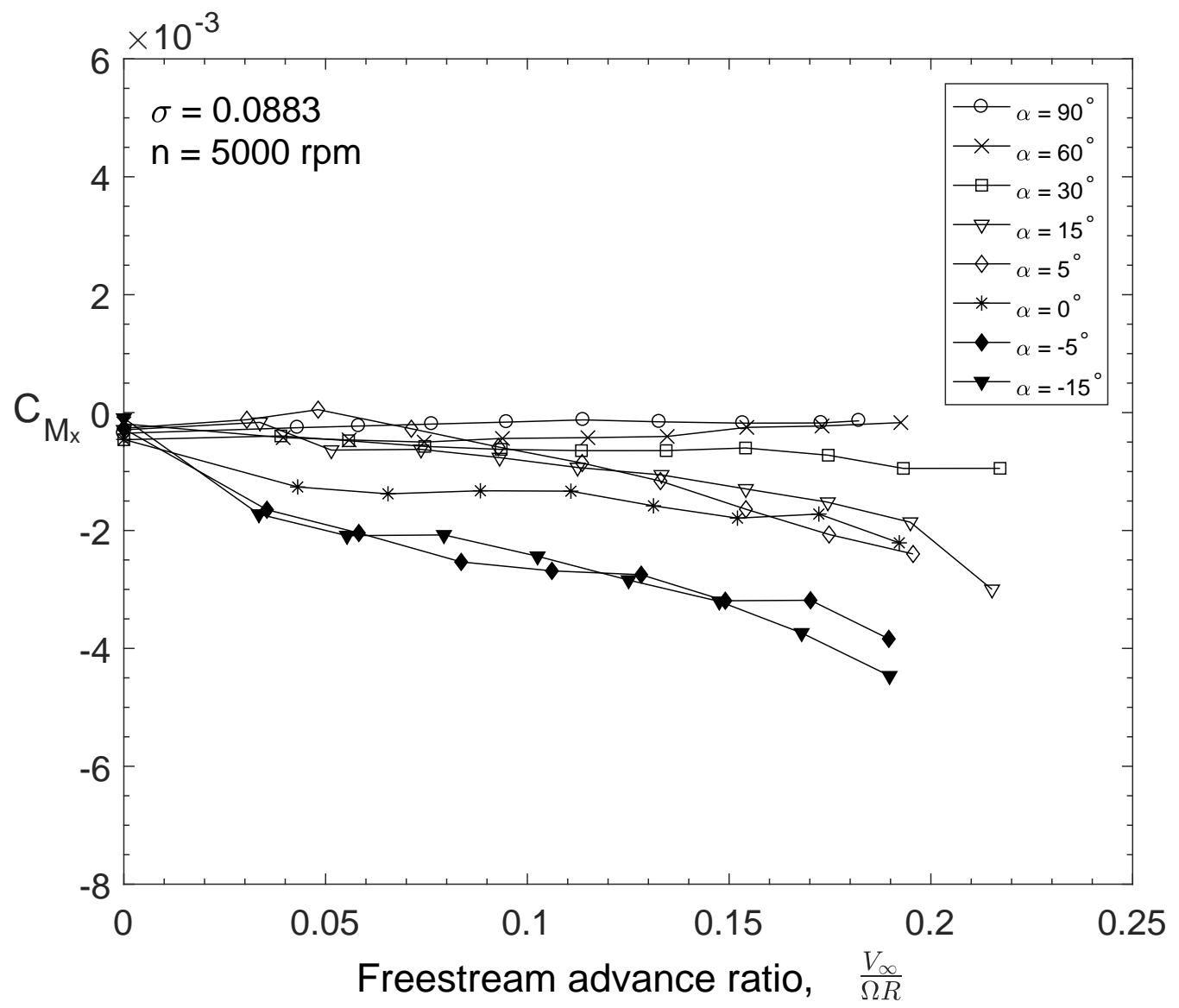

Figure A.3.9: $C_{M_{x}}$ versus advance ratio for the T-motor 18x6.1, RPM $=5000$ 


\section{Appendix B}

\section{B.1 Test Stand Components}

The components used for the experimental apparatus are listed in Tables B.1.1 and B.1.2.

\begin{tabular}{|c|c|c|}
\hline Component Function & Qty & Component Name and Description \\
\hline Power supply & 1 & $\begin{array}{l}\text { Hitec ePowerbox 15-30V 1200W 50A AC/DC } \\
\text { power supply }\end{array}$ \\
\hline Electronic speed controller & 1 & Scorpion Commander 50V 90A ESC (OPTO) \\
\hline Brushless motor & 1 & Scorpion 511-4020-420KV $1500 \mathrm{~W}$ \\
\hline Load cell (150lbs) & 1 & A-Tech MLP-150 Low-profile load cell \\
\hline Load cell (50 lbs) & 2 & A-Tech MLP-50 Low-profile load cell \\
\hline Load cell conditioner & 3 & $\begin{array}{l}\text { Omega DMD-460 Bridge amplifier/signal } \\
\text { conditioner (including power chord and rail } \\
\text { mounting kit DIN400) }\end{array}$ \\
\hline $\begin{array}{l}\text { Differential pressure } \\
\text { transducer }\end{array}$ & 1 & $\begin{array}{l}\text { Omega Px277-0.1D5V differential pressure } \\
\text { transmitter with selectable ranges }\end{array}$ \\
\hline $\begin{array}{l}\text { Absolute pressure } \\
\text { transducer }\end{array}$ & 1 & $\begin{array}{l}\text { Valindyne P55A General purpose pressure } \\
\text { transducer and transmitter }\end{array}$ \\
\hline Infrared sensor & 1 & $\begin{array}{l}\text { E18-D80NK 5V DC diffuse reflective type infrared } \\
\text { sensor }\end{array}$ \\
\hline Current and Voltage Sensor & 1 & $\begin{array}{l}\text { AttoPilot SEN-10644 Voltage and Current Sense } \\
\text { Breakout - 180A }\end{array}$ \\
\hline Temperature probe & 1 & LabJack EI 1034 Temperature probe \\
\hline Terminal board & 1 & LabJack CB37 Terminal board \\
\hline
\end{tabular}

Table B.1.1: Components used for the rotor test stand. 


\begin{tabular}{|l|c|l|}
\hline Component & Qty & Component Name and Description \\
\hline \hline Data Acquisition System & 1 & $\begin{array}{l}\text { LabJack T7 USB/Ethernet multifunction analog } \\
\text { DAQ }\end{array}$ \\
\hline $\begin{array}{l}\text { Microcontroller and Digital/Analog } \\
\text { Data Acquisition System }\end{array}$ & 1 & $\begin{array}{l}\text { Arduino UNO Multifunction microcontroller and } \\
\text { DAQ }\end{array}$ \\
\hline
\end{tabular}

Table B.1.2: Components used for the rotor test stand data acquisition system.

\section{B.1.1 Test Stand Uncertainty Analysis}

An uncertainty analysis was performed on the test stand components and is summarized in Tables B.1.3 and B.1.4. The uncertainty analysis is courtesy of Dylan Krcmarov.

\begin{tabular}{|l|l|}
\hline Component & Uncertainty \\
\hline \hline Atmospheric Pressure & $0.2172 \%$ \\
\hline Differential Pressure & $1.5031 \%$ \\
\hline Load Cells & $0.6883 \%$ \\
\hline RPM Sensor & $0.4911 \%$ \\
\hline Thermocouple & $1.3018 \%$ \\
\hline
\end{tabular}

Table B.1.3: Uncertainty analysis summary for the test stand sensors.

\begin{tabular}{|l|l|}
\hline Measurement & Uncertainty \% \\
\hline \hline Air Density & $1.3198 \%$ \\
\hline Air Temperature & $1.3018 \%$ \\
\hline Rotational Speed & $0.4911 \%$ \\
\hline Wind Tunnel Airspeed & $1.0001 \%$ \\
\hline Thrust/Torque/Pitch & $0.6883 \%$ \\
\hline Power & $0.8455 \%$ \\
\hline Thrust/Torque/Pitch Coefficient & $1.7834 \%$ \\
\hline Power Coefficient & $2.1511 \%$ \\
\hline Efficiency & $3.0082 \%$ \\
\hline
\end{tabular}

Table B.1.4: Uncertainty analysis summary for measured quantities. 


\section{B.2 Ryerson University's Low Speed Wind Tunnel}

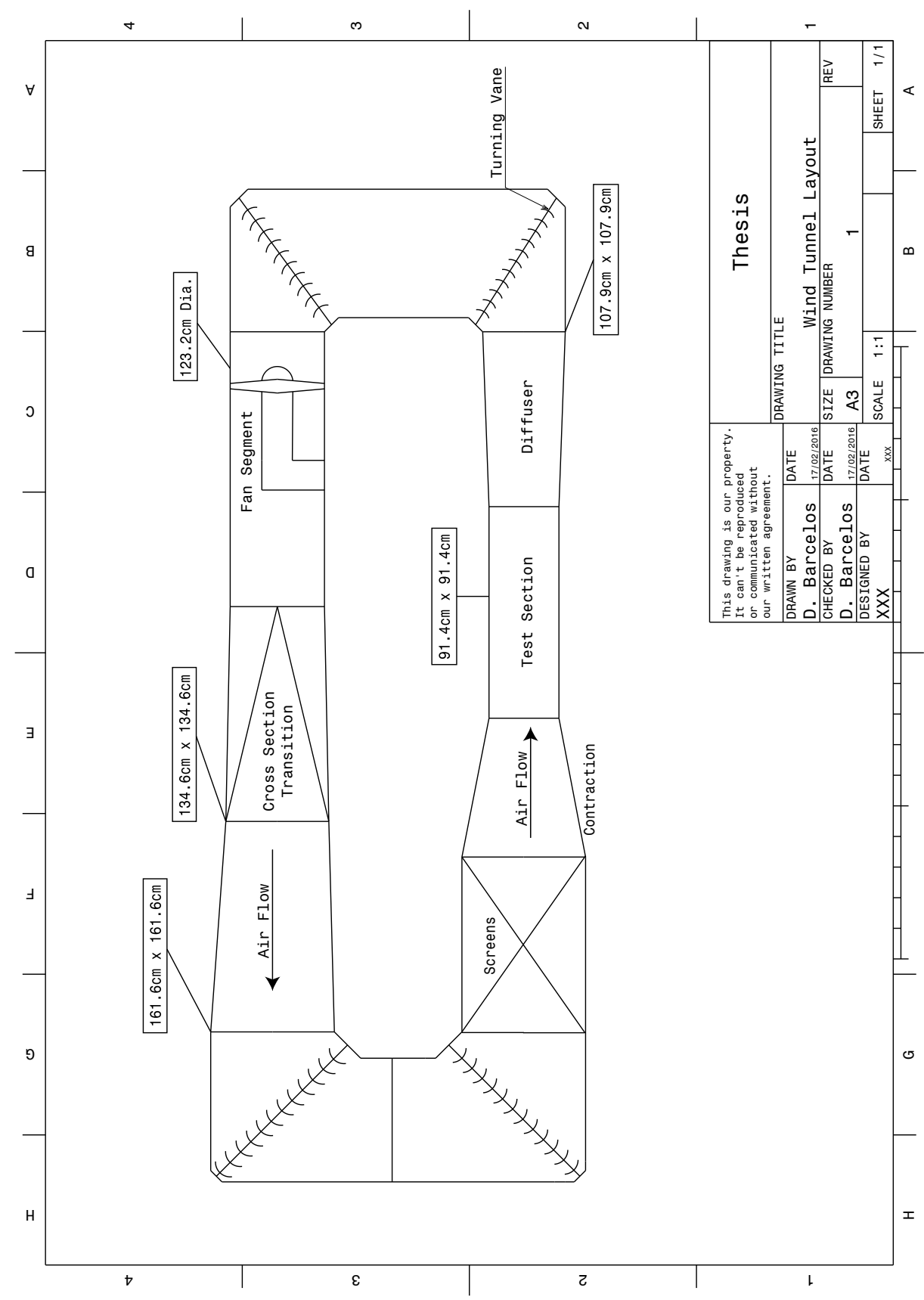

Figure B.2.1: Schematic of the RU closed circuit wind tunnel. (Courtesy of Devin Barcelos) 


\section{Appendix C}

\section{C.1 Analytical Solution to the Single Rotor Flow Field}

The velocities induced by a vortex tube for a single rotor flow field, from the analytical solution using elliptical integrals in Ref.53, are shown in Figs.C.1.1 through C.1.6.

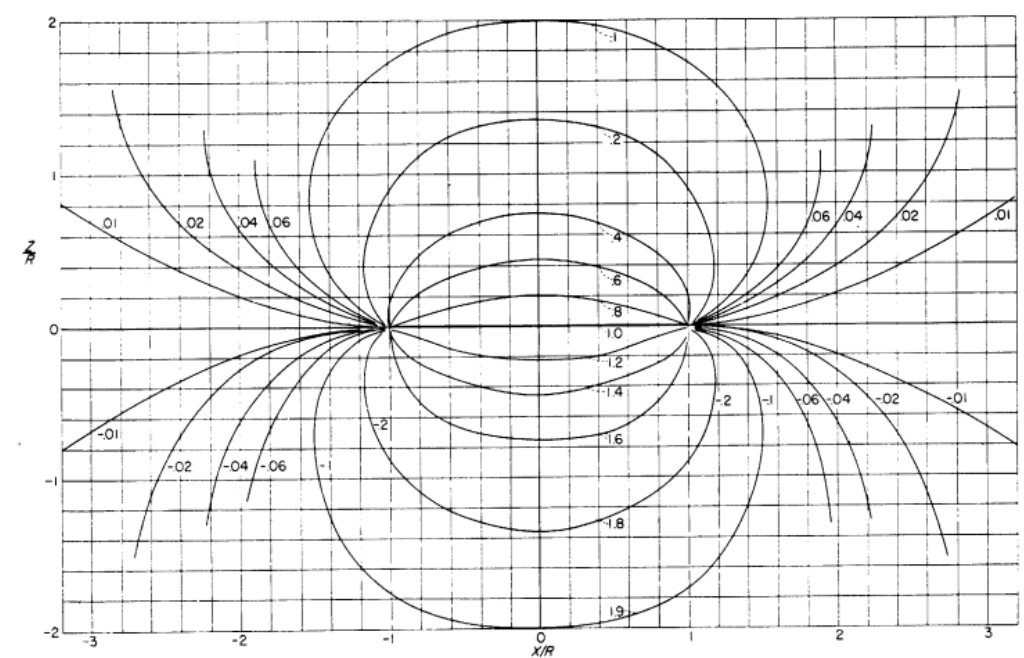

Figure C.1.1: Lines of constant induced velocity ratio $\frac{V_{i}}{v}$ for $\chi=0^{\circ}$ using the analytical solution in Ref.53. 


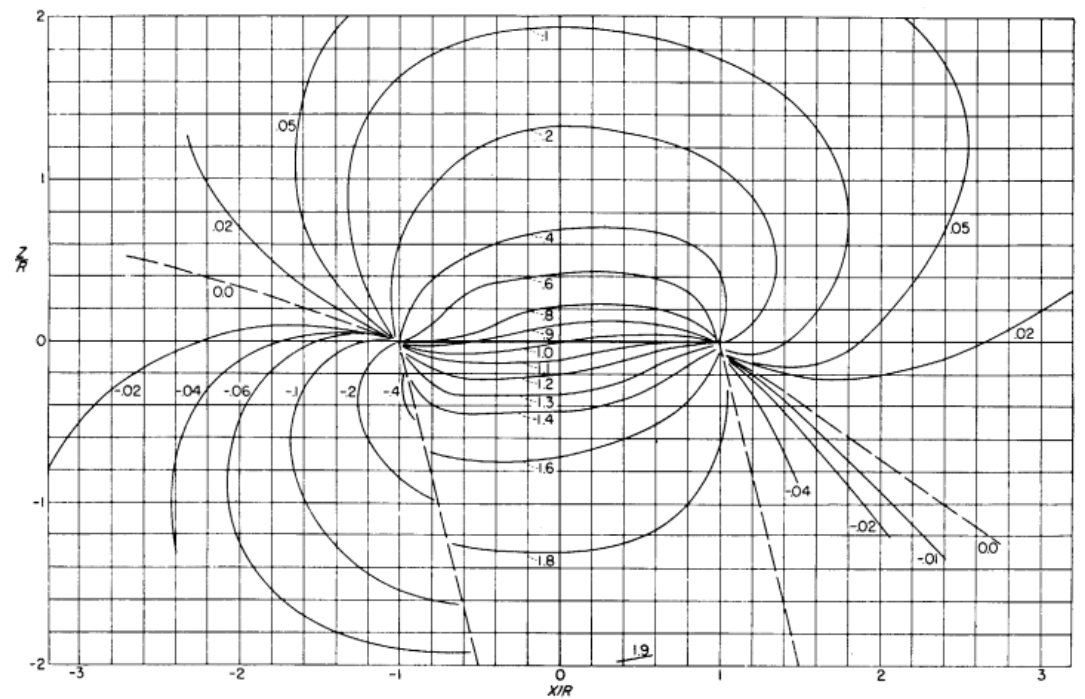

Figure C.1.2: Lines of constant induced velocity ratio $\frac{V_{i}}{v}$ for $\chi=14.04^{\circ}$ using the analytical solution in Ref.53.

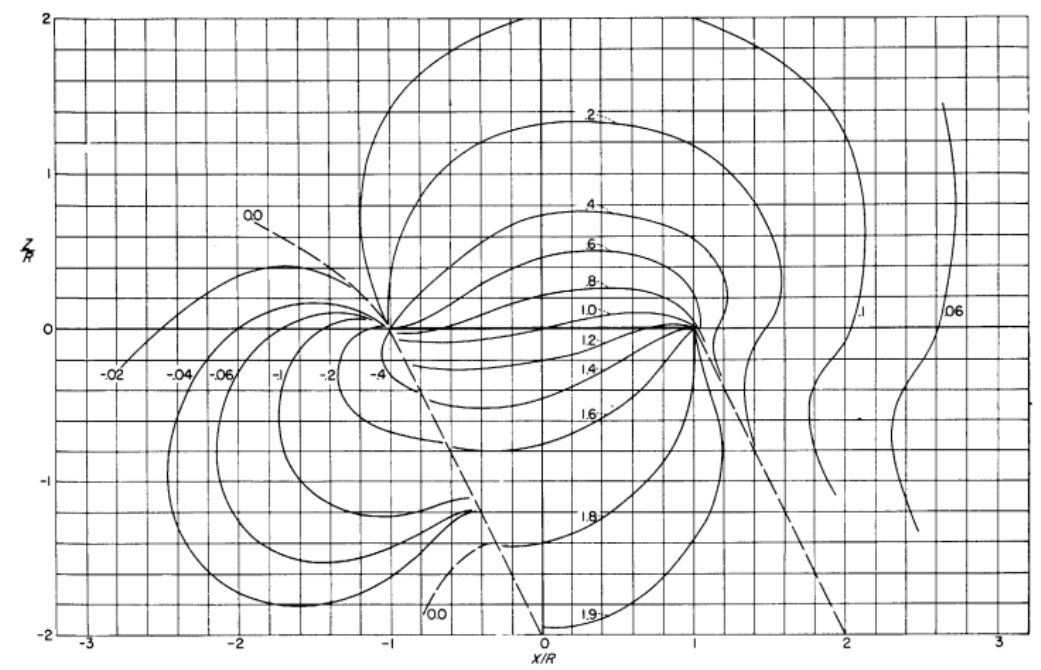

Figure C.1.3: Lines of constant induced velocity ratio $\frac{V_{i}}{v}$ for $\chi=26.56^{\circ}$ using the analytical solution in Ref.53. 


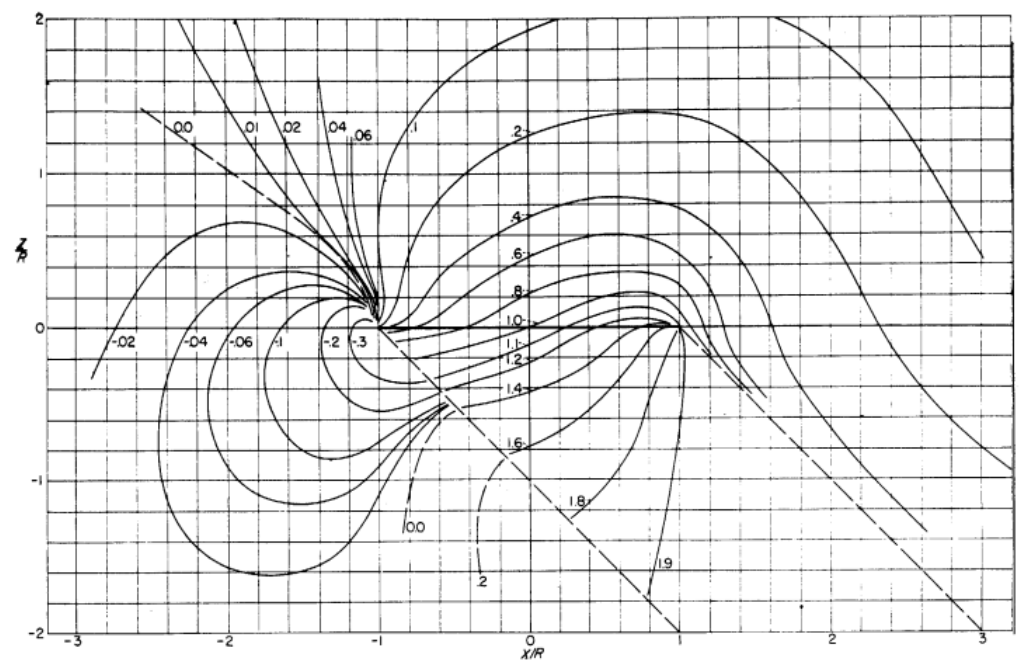

Figure C.1.4: Lines of constant induced velocity ratio $\frac{V_{i}}{v}$ for $\chi=45^{\circ}$ using the analytical solution in Ref.53.

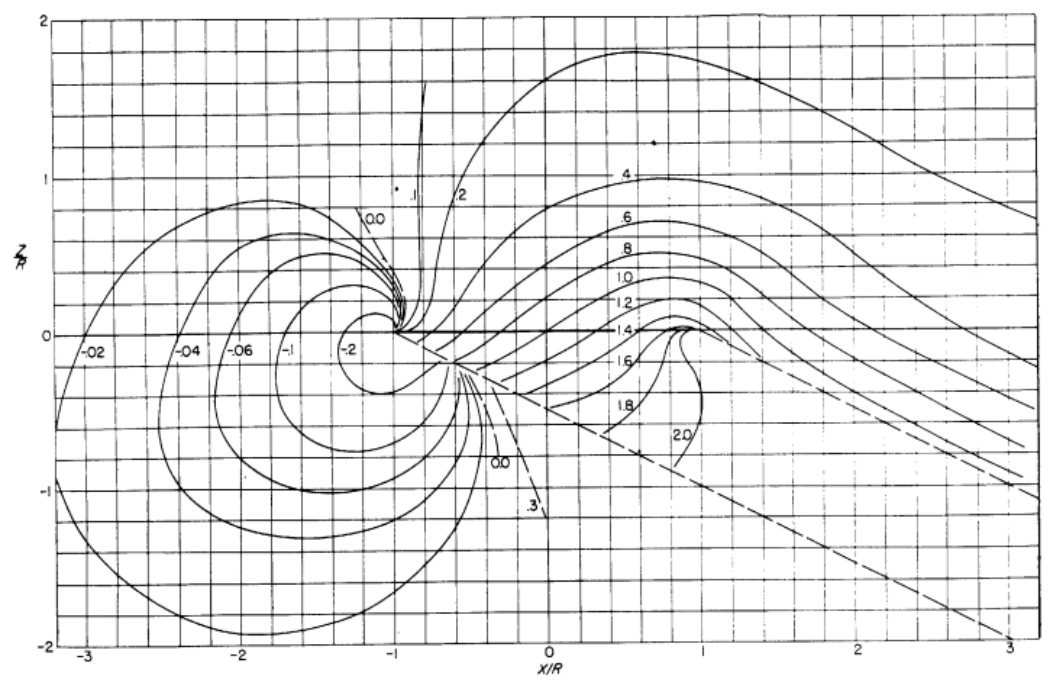

Figure C.1.5: Lines of constant induced velocity ratio $\frac{V_{i}}{v}$ for $\chi=63.43^{\circ}$ using the analytical solution in Ref.53. 


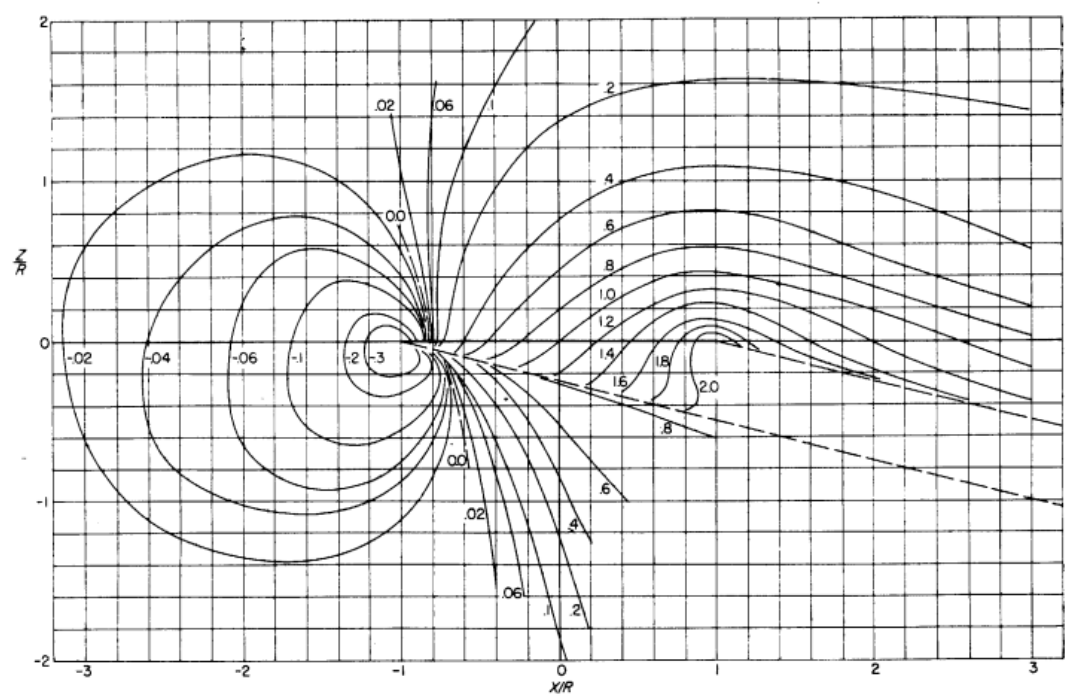

Figure C.1.6: Lines of constant induced velocity ratio $\frac{V_{i}}{v}$ for $\chi=75.97^{\circ}$ using the analytical solution in Ref.53. 


\section{References}

[1] Logan, M. J., Chu, J., Motter, M. A., Carter, D. L., Ol, M., and Zeune, C., "Small uav research and evolution in long endurance electric powered vehicles," in AIAA Infotech @ Aerospace 2007 Conference and Exhibit, vol. 2730, 2007, pp. 7-10.

[2] Gupte, S., Mohandas, P. I., and Conrad, J. M., "A survey of quadrotor unmanned aerial vehicles," in Southeastcon, 2012 Proceedings of IEEE, 2012, pp. 1-6.

[3] Aeryon Labs Inc., "Aeryon Skyranger," Retrieved from https://www.aeryon.com/wpp/ wp-content/files/brochures/Aeryon-SkyRanger-Brochure.pdf, 2016, online; accessed 7 June 2016.

[4] Gustafson, F.B., and Gessow, A., "Effect of rotor-tip speed on helicopter hovering performance and maximum forward speed," National Advisory Committee for Aeronautics ACR L6A16, Tech. Rep., 1946.

[5] Keys, C., Tarzanin, F., and McHugh, F., "Effects of twist on helicopter performance and vibratory loads," in 13th European Rotorcraft Forum., Arles, France, 1987.

[6] Yang, Z., Sankar, L. N., Smith, M. J., and Bauchau, O., "Recent improvements to a hybrid method for rotors in forward flight," Journal of Aircraft, vol. 39, no. 5, pp. 804-812, 2002.

[7] Liiva, J., "Unsteady aerodynamic and stall effects on helicopter rotor blade airfoil sections," Journal of Aircraft, vol. 6, no. 1, pp. 46-51, 1969.

[8] Chattopadhyay, A.,McCarthy, T.R., and Pagaldipti, N., "Multilevel decomposition procedure for efficient design optimization of helicopter rotor blades," AIAA Journal, vol. 33, no. 2, pp. 223-230, 1995.

[9] User:Commanderbryce/Wikimedia Commons/CC-BY-SA-3.0, "Minion 220 racing multirotor drone by RotorGeeks," Retrieved from https://commons.wikimedia.org/wiki/File: Racing_Drone.jpg, 2016, online; accessed 2 October 2016. 
[10] Aiken, E. W., Ormiston, R. A., and Young, L., A., "Future directions in rotorcraft technology at ames research center," in 56th Annual Forum of the American Helicopter Society, Virginia Beach, VA, 2000.

[11] Pines, D. J., and Bohorquez, F., "Challenges facing future micro-air-vehicle development," Journal of Aircraft, vol. 43, no. 2, pp. 290-305, 2006.

[12] Young, L. A., Aiken, E.W., Johnson, J.L., Demblewski, R., Andrews, J., and Klem, J., "New concepts and perspectives on micro-rotorcraft and small autonomous rotary-wing vehicles," in AIAA 20th Applied Aerodynamics Conference, 2002.

[13] Leishman, G. J., Principles of Helicopter Aerodynamics. Cambridge University Press, 2006.

[14] Bohorquez, F., "Rotor hover performance and system design of an efficient coaxial rotary wing micro air vehicle," Ph.D. dissertation, University of Maryland, College Park, 2007.

[15] Bohorquez, F., Pines, D., and Samuel, P. D., "Small rotor design optimization using blade element momentum theory and hover tests," Journal of Aircraft, vol. 47, no. 1, pp. 268-283, 2010.

[16] Sodja, J., Drazumeric, R., Kosel, T., and Marzocca, P., "Design of flexible propellers with optimized load-distribution characteristics," Journal of Aircraft, vol. 51, no. 1, pp. 117-128, 2014.

[17] M. S. Selig et al., Summary of Low Speed Airfoil Data. Virginia, VA: SoarTech Publications, 1995, vol. 1-5.

[18] R. Randall, C.-A. Hoffmann, and S. Shkarayev, "Longitudinal aerodynamics of a vertical takeoff and landing micro air vehicle," Journal of Aircraft, vol. 48, no. 1, pp. 166-176, 2011.

[19] Tsadok, T., "Thruster modeling for small unmanned aerial vehicles with coaxial-rotors," Master's thesis, McGill University, Mechanical Engineering Dept., Montreal, QC, 2014.

[20] Betz, A., "Schraubenpropeller mit geringstem energieverlust. mit einem zusatz von 1. prandtl," vol. 2, p. 193-217, 1919, Screw Propeller with Minimum Energy Loss, Technical Translation 736, translated by D. A. Sinclair, National Research Council Library, Canada, 1958.

[21] Munk, M., "The minimum induced drag of aerofoils," NACA Report 121, 1923. 
[22] Goldstein, S., "On the vortex theory of screw propellers," Proceedings of the Royal Society of London. Series A, Containing Papers of a Mathematical and Physical Character, pp. 440-465, 1929.

[23] Theodorsen, T., Theory of propellers. McGraw-Hill New York, 1948, vol. 3.

[24] Moffitt, R.C., and Bissell, J. R., "Theory and application of optimum airloads to rotors in hover and forward flight," in Proceedings of the 38th of the Annual Forum of the American Helicopter Society, 1982.

[25] Landgrebe, A. J., "An analytical and experimental investigation of helicopter rotor hover performance and wake geometry characteristics," U.S. Army Air Mobility Research and Development Laboratory, USAAMRDL Technical Report 71-24, June 1971.

[26] Froude,W., "On the elementary relation between pitch, slip and propulsive efficiency," Transactions Institute of Naval Architects, no. 19, pp. 47-57, 1878.

[27] Rankine, W. J. M., On the Mechanical Priciples of the Action of Propellers. Transaction of the Institute of Naval Architects, 1865.

[28] Glauert, H., "Airplane propellers," in Aerodynamic theory. Springer, 1935, pp. 169-360.

[29] Gessow, A., "Effect of rotor-blade twist and plan-form taper on helicopter hovering performance," National Advisory Committee for Aeronautics, Langley Aeronautical Lab., Tech. Rep., 1948.

[30] Larrabee, E., and French, S. E., "Minimum induced loss windmills and propellers," Journal of Wind Engineering and Industrial Aerodynamics, vol. 15, no. 1, pp. 317-327, 1983.

[31] Adkins, C. N., and Liebeck, R. H., "Design of optimum propellers," Journal of Propulsion and Power, vol. 10, no. 5, pp. 676-682, 1994.

[32] Bennett, R. L., "Application of optimization methods to rotor design-problems," Vertica, vol. 7, no. 3, pp. 201-208, 1983.

[33] McCormick, B. W., Aerodynamics, aeronautics, and flight mechanics. New York: Wiley, 1995, vol. 2.

[34] Walsh, J. L., Bingham, G. J., and Riley, M. F., "Optimization methods applied to the aerodynamic design of helicopter rotor blades," Journal of the American Helicopter Society, vol. 32, no. 4, pp. 39-44, 1987. 
[35] Kroninger, C. M., "Characteristics of a rotor optimized for hover and forward flight," Master's thesis, The Pennsylvania State University, 2008.

[36] Chattopadhyay, A., and McCarthy, T. R., "Multiobjective design optimization of helicopter rotor blades with multidisciplinary couplings," Optimization of Structural Systems and Industrial Applications, pp. 451-462, 1991.

[37] Lee, J., and Hajela, P., "Parallel genetic algorithm implementation in multidisciplinary rotor blade design," Journal of Aircraft, vol. 33, no. 5, pp. 962-969, 1996.

[38] Bohorquez, F., Samuel, P., Sirohi, J., Pines, D., Rudd, L., and Perel, R., "Design, analysis and hover performance of a rotary wing micro air vehicle," Journal of the American Helicopter Society, vol. 48, no. 2, pp. 80-90, 2003.

[39] Bohorquez, F., and Pines, D., "Hover performance of rotor blades at low reynolds numbers for rotary wing micro air vehicles. an experimental and cfd study," 21st AIAA Applied Aerodynamics Conference, Orlando, FL, June 2003.

[40] Brandt, J., "Small-scale propeller performance at low speeds," Master's thesis, University of Illinois at Urbana Champaign, 2005.

[41] Burger, C., "Propeller performance analysis and multidisciplinary optimization using a genetic algorithm," Ph.D. dissertation, Auburn University, 2007.

[42] Gur, O., and Rosen, A., "Optimization of propeller based propulsion system," Journal of Aircraft, vol. 46, no. 1, pp. 95-106, 2009.

[43] Stahlhut, C. W., "Aerodynamic design optimization of proprotors for convertible-rotor concepts," Master's thesis, University of Maryland, College Park, 2012.

[44] Khan, W., and Nahon, M., "A propeller model for general forward flight conditions," International Journal of Intelligent Unmanned Systems, vol. 3, pp. 72-92, 2015.

[45] Roskam, J., and Lan, C. E., Airplane Aerodynamics and Performance. Design, Analysis, and Research Corporation, 1997.

[46] Chen, R. T. N., "A survey of nonuniform inflow models for rotorcraft flight dynamics and control applications," NASA Technical Memorandum 102219, vol. 14, 1989.

[47] Coleman, T.,F and Li, Y., "An interior trust region approach for nonlinear minimization subject to bounds," SIAM Journal on optimization, vol. 6, no. 2, pp. 418-445, 1996. 
[48] Maniaci, D. C., "An investigation of wt_perf convergence issues," in 49th AIAA Aerospace Sciences Meeting including the New Horizons Forum and Aerospace Exposition, 2011, pp. 2011-150.

[49] Ning, S. A., "A simple solution method for the blade element momentum equations with guaranteed convergence," Wind Energy, vol. 17, no. 9, pp. 1327-1345, 2014.

[50] Choephel,T, "Aerodynamic analysis of helicopter rotors using a higher-order, free-wake method," Ph.D. dissertation, The Pennsylvania State University, 2016.

[51] Coleman, R. P., Feingold, A. M., and Stempin, C. W., "Evaluation of the induced-velocity field of an idealized helicoptor rotor," NACA ARR L5E10, Tech. Rep., 1945.

[52] Drees, J. M., "A theory of airflow through rotors and its application to some helicopter problems," Journal of the Helicopter Association of Great Britain, vol. 3, no. 2, pp. 79-104, 1949.

[53] Castles, W., and De Leeuw, J. H., "The normal component of the induced velocity in the vicinity of a lifting rotor and some examples of its application," 1954.

[54] Pitt, D. M., and Peters, D. A., "Theoretical prediction of dynamic-inflow derivatives," Vertica, vol. 5, no. 1, pp. 21-34, 1981.

[55] Johnson, W., Helicopter theory. Princeton University Press, 1980.

[56] Drela, M., "Xfoil: An analysis and design system for low reynolds number airfoils." University of Notre Dame: Conference on Low Reynolds Number Airfoil Aerodynamics, 1989, pp. 1-12.

[57] Lindenburg, C., "Stall coefficients," in NREL Rept. ECN-RX-01-004, IEA Symposium on the Aerodynamics of Wind Turbines, Energy Research Centre of the Netherlands. National Renewable Energy Laboratory, 2000.

[58] Lindenburg, C, "Investigation into rotor blade aerodynamics," Netherlands Society for Energy and the Environment, Paper ECN-C-03-025, 2003.

[59] Hoerner, S. F., and Borst, H. V., "Fluid-dynamic lift: Practical information on aerodynamic and hydrodynamic lift," NASA STI/Recon Technical Report A, vol. 76, p. 32167, 1975.

[60] George,I, “A multirotor vehicle performance prediction method," Master's thesis, Ryerson University, 2016. 
[61] Katz, J. and Plotkin, A., Low-Speed Aerodynamics, 13th ed. Cambridge University Press, New York, 2001.

[62] Barcelos, D, "Flow quality testing and improvement of the ryerson university low speed wind tunnel," Ryerson University, Undergraduate Thesis, April 2016.

[63] Brandt, J. B., Deters, R. W., Ananda, G. K., and Selig, M. S., "UIUC propeller database," University of Illinois at Urbana-Champaign, retrieved from http://m-selig.ae.illinois.edu/ props/propDB.html.

[64] Eberhart, R. C., and Kennedy, J., "A new optimizer using particle swarm theory," in Proceedings of the Sixth International Symposium on Micro Machine and Human Science. IEEE, 1995, pp. 39-43.

[65] Golberg, D. E., "Genetic algorithms in search, optimization, and machine learning," Addion wesley, vol. 1989, p. 102, 1989.

[66] Hassan, R., Cohanim, B., De Weck, O., and Venter, G., "A comparison of particle swarm optimization and the genetic algorithm," in Proceedings of the 1st AIAA multidisciplinary design optimization specialist conference, 2005, pp. 18-21. 